Final

Report

October 1972

\title{
Space Storable Propellant Acquisition System
}

(NASA-CR-114493) SPACE STORABLE PROPELLANT
iCQUISITION SYSTEM J.R. TEqart, et al
(Martin Marietta Corp.)

(Martin Marietta Corp.) Oct. 1972 al $287 \mathrm{p}$

N 72-33864

Unclas

G3/31 44732

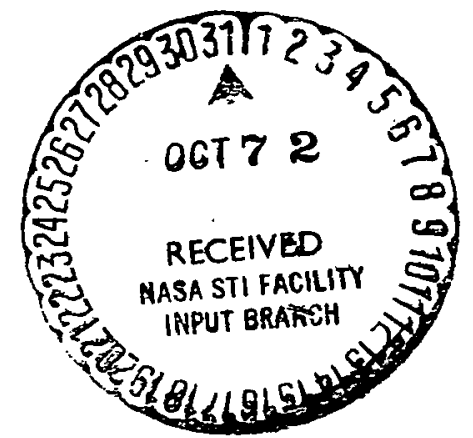




\author{
SPACE STORABLE \\ PROPELLANT ACQUISITION \\ SYSTEM
}

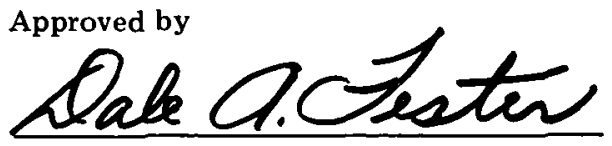

Dale A. Fester

Program Manager

Prepared for

Jet Propulsion Laboratory

California Institute of Technology

Pasadena, California 91103

Prepared by

J. R. Tegart

P. E. Uney

J. E. Anderson

D. A. Fester

MARTIN MARIETTA AEROSP.ACE

P.O. Box 179

Denver, Colorado 80201 
This report is submitted by Martin Marietta Aerospace in accordance with Article IV.A and Attachment B of Contract NAS2-6548, dated 25 June 1971.

The work was administered under the technical direction of $\mathrm{Mr}$. George Yankura, JPL Technical Manager. Mr. Frank Stevenson of NASA Headquarters was the NASA Project Manager, and Mr. Martin Gross and Mr. Frank DeRosa of NASA-ARC were responsible for procurement and contract administration, respectively. Mr. Dale Fester, Thermodynamics and Fluid Mechanics Section, Propulsion Department, was the Martin Marietta Program Manager.

The following Martin Marietta personnel made significant contributions to the program:

James R. Tegart Fluid Mechanics Analysis and Surface Tension Device Configuration

Preston E. Uney Experimental Program Direction, Fabrication and Installation Considerations, and Detailed Design

John E. Anderson Pressurization Analysis and Comparative Evaluations

Ralph E. Hise Program Criteria, Spacecraft Accelerations and Center of Gravity

Paul J. Grosser Detailed Design 
$\underline{\text { Page }}$

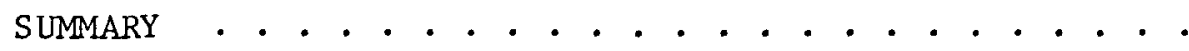

viị

I. INTRODUCTION . . . . . . . . . . . . . . . . . . . II-1

A. Program Background and Objectives . . . . . . . . . I I-1

B. Mission and Spacecraft Criteria . . . . . . . . . . I-2

C. Study Guidelines . . . . . . . . . . . . . . I I-10

D. Approach . . . . . . . . . . . . . . . I-12

thru

$\mathrm{I}-15$

II. CANDIDATE PROPELLANT ACQUISITION CONCEPTS . . . . . . . II-1

A. Category A Devices . . . . . . . . . . . . . . . . . II-1

B. Category B Devices . . . . . . . . . . . . . . . II-3

C. Category C Devices . . . . . . . . . . . . . . II-4

D. Preliminary Screening . . . . . . . . . . . . . II-7

and

II-8

III. ANALYSIS OF CANDIDATE CONCEPTS . . . . . . . . . . . . III-1

A. System Operational Considerations . . . . . . . . . . . III-1

B. Subsystem Interactions . . . . . . . . . . . . . III-76

C. System Fabrication, Assembly and Tank Installation . . . III-103

...thru

III -123

IV. EXPERIMENTAL EVALUATION PROGRAM . . . . . . . . . . . IV-1

A. Test Plan . . . . . . . . . . . . . . . . . . . . IV IV-1

B. Test Apparatus . . . . . . . . . . . . . . . . IV 9

C. Test Results . . . . . . . . . . . . . . . . IV-12

thru

IV -24

V. COMParative eVAlutition . . . . . . . . . . . . . . V V-1

A. Propellant Acquisition System Evaluation . . . . . . . . V-1

B. Propellant Combination Comparison . . . . . . . . . V-15

thru

$\mathrm{V}-37$

VI. DETAIL DESIGN OF SELECTED SYSTEM . . . . . . . . . . . V VI-1

A. System Design . . . . . . . . . . . . . . . . . V VI-2

B. Design Analysis . . . . . . . . . . . . . . . VI-30

C. Other System Considerations . . . . . . . . . . . VI-51

thru

VI-56 
VII. CONCLUSIONS AND RECOMMENDATIONS . . . . . . . . . . . . VII-1

A. Conclusions . . . . . . . . . . . . . . . . . . . VII-1

B. Recommendations . . . . . . . . . . . . . VII-2

and

VII -3

VIII. REFERENCES .................... . . VIII-1

thru

VIII-5

Figure

I-1 Sun-Spacecraft Orientation During Mission . . . . . . I-3

I-2 Flox/MMH Propulsion Subsystem . . . . . . . . . . I I-8

I-3 $\mathrm{F}_{2} / \mathrm{N}_{2} \mathrm{H}_{4}$ Dual-Mode Propulsion Subsystem . . . . . . . . I-11

I-4 Program Approach . . . . . . . . . . . . . . I-13

II-1 Category A Devices . . . . . . . . . . . . . II-2

II-2 Category B Devices . . . . . . . . . . . . . . II-3

II-3 Category C Devices . . . . . . . . . . . . . . . II-5

II-4 Category D Device Combinations . . . . . . . . . . . II-6

II-5 Candidate Devices . . . . . . . . . . . . . . . II-8

III-1 Compartmented Trap . . . . . . . . . . . . . . III-2

III-2 Cruciform . . . . . . . . . . . . . . . . . III-5

III-3 Principal Radii of Curvature . . . . . . . . . . III-6

III-4 Zero-g Interface for the Cruciform . . . . . . . . . III-7

III-5 Post for Oxidizer Tank . . . . . . . . . . . III-10

III-6 Post for Fuel Tank . . . . . . . . . . . . . . III-10

III-7 Post Configurations . . . . . . . . . . . . . III-11

III-8 Post Height vs Ullage Volume . . . . . . . . . . III-13

III-9 Interface Configurations . . . . . . . . . . . . III-14

III-10 Stability of Liquid About a Cylindrical Fruhof Post in

III-11 Effect of a Stuck Interface on Stability . . . . . . . III-18

III-12 Low-g Interfaces about Post. . . . . . . . . . III-19

III-13 Standpipe for Oxidizer Tank . . . . . . . . . . . . III-21

III-14 Standpipe for Fuel Tank . . . . . . . . . . . . . . III-21

III-15 Positioning of a 5\% Bubble . . . . . . . . . . . . III-23

III-16 Equilibrium Configuration for the Standpipe. . . . . III-25

III-17 Post with Fingers for Oxidizer Tank . . . . . . . . . III-27

III-18 Post with Fingers for Fuel Tank. . . . . . . . . . III-27

III-19 Vanes for Oxidizer Tank . . . . . . . . . . . . III-29

III-20 Vanes for Fuel Tank . . . . . . . . . . . . . . . III-29

III-21 Vane Profile Limits . . . . . . . . . . . . . . III-30

III-22 Inner Profile Limit for 5\% Ullage. . . . . . . . . III-31

III-23 Outer Profile Limit . . . . . . . . . . . . III-33 
III-24 Vane Profile at Outlet . . . . . . . . . . .

III -25

III -26

III -27

III -28

III -29

III -30

III -31

III -32

III-33

III -34

III -35

III -36

III -37

III -38

III-39

III -40

III-41

III -42

III -43

III -44

III -45

III -46

III -47

III -48

III-49

III -50

III-5I

III -52

III -53

III -54

III-55

III -56

III -57

III -58

III-59

III -60

III-61

III -62

III -63

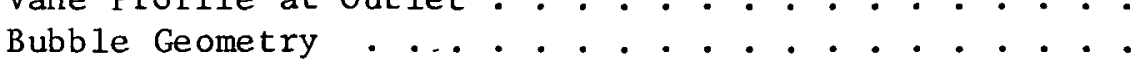

Pumping Capability vs Ullage Volume . . . . . . . .

Six-Vane Device Profile . . . . . . . . . . . .

Eight-Vane Device Profile . . . . . . . . . .

12-Vane Device Profile.. . . . . . . . . . . .

Ullage Bubble Displacement vs Time . . . . . . . .

Standpipe with Vanes for Oxidizer Tank . . . . . . . .

Standpipe with Vanes for Fuel Tank . . . . . . . .

Curvature vs Volume for Zero Contact Angle . . . . . .

Curvature vs Volume for $5^{\circ}$ Contact Angle ....... .

Equilibrium Configuration for $5^{\circ}$ Contact Angle . . . .

Comparison of Curvature for Three Devices at $5^{\circ}$ Con-

tact Angle . . . . . . . . . . . . . . . . .

Basic Trajectory Correction Maneuver Sequence . . .

First Mode Harmonic Frequency for Spherical Tank . .

Reference Spacecraft Propellant Tank Envelope . . .

In-Line Tank Arrangement for Flox/MMH . . . . . . .

In-Line Tank Arrangement for $\mathrm{F}_{2} / \mathrm{N}_{2} \mathrm{H}_{4}$. . . . . . . . .

Side-by-Side Tank Arrangement for Flox/MMH . . . . .

Side-by-Side Tank Arrangement for $\mathrm{F}_{2} / \mathrm{N}_{2} \mathrm{H}_{4}$. . . . . .

In-Line Tank Arrangement cg Shift . . . . . . . .

Side-by-Side Tank Arrangement cg Shift . . . . . . .

Rise Rate vs Bubble Radius for Low M Liquids . . . .

Flow Velocity Profile . . . . . . . . . . . .

Bubble Equilibrium Conditions for Flox . . . . . .

Bubble Equilibrium Conditions for MMH . . . . . . .

Initial Conditions for Marker and Cell Computations .

Marker and Ce11 Solution to Propellant Draining

Problem . . . . . . . . . . . . . . .

Propellant Tank Pressure Histories . . . . . . . .

Flox/MMH Propulsion Subsystem . . . . . . . . . .

Propellant Tank Pressure History for 60-Second Pre-

pressurization Duration . . . . . . . . . .

Flox Tank Support Structure . . . . . . . . . .

Circular Fin Simulation of Spherical Propellant Tank.

Wa1l Temperature Distribution Near Tank Support . . .

Wa11 Temperature Distribution Near Tank Outlet . . .

Circular Fin Simulation of Propellant Tank . . . . . .

Propellant Tank Wall Temperature Profiles . . . . .

Installation of Four-Vaned Acquisition Device by

Rolling . . . . . . . . . . . . . . . . . .

Maximum Vane Curvature Occurring during Installation of Acquisition Device . . . . . . . . . . . . . .

Maximum Allowable Vane Thickness to Install Acquisition Device
III -34

III -36

III-36

III-38

III -38

III -38

III -40

III -44

III-44

III-48

III -50

I II -52

III -53

III-56

III -58

III -60

I II-62

III -62

III-63

III-63

III-64

III-64

III-69

III-69

III -70

III-70

III -72

III -73

III -79

III-80

III -83

III- 88

III-91

III-94

III -96

III-97

III -100

III-104

III -106

III-108 
III-64 Approximated Finger Shape used in Bending Analysis .

III -109

IV-1

Device Set A

IV-3

IV-2

IV -4

IV-3

Device Set $C$

IV -5

IV-4

Device Set D

IV -6

IV-5

Device Set E

IV-6

Device Set F

IV -7

IV -7

Test Fixtur

IV -8

IV-8

Test Fixture with Devices Installed.

IV -10

IV-9

Vane-Type Device Models

IV -11

IV -10

Device Set A Tests

IV -11

IV -11

Device Set B Tests

IV -12

Device Set $C$ Tests

. . . . .

IV -13

IV -15

IV -16

IV -13

Device Set D Tests

IV -17

IV-14

IV -15

Device Set E Tests

IV -18

Device Set $\mathrm{F}$ Tests

IV-19

$\mathrm{V}-1$

Effect of Temperature and Contact Angle on Oxidizer Interface Stability - $\cdot \dot{0} \cdot \cdot \cdot \cdot \cdot \cdot \cdot \cdot \cdot \dot{C}^{\circ} \cdot \cdot \cdot \cdot$

V-2 Effect of Temperature and Contact Angle on Fue1 Interface Stability

$\mathrm{V}-20$

V-3 Effect of Temperature and Contact Angle on Oxidizer Capillary Pumping . . . . . . . . . . . . . . . . .

V-4 Effect of Temperature and Contact Angle on Fuel Capil1ary Pumping

Ullage Fraction Variation with Temperature for Oxidizers

Ullage Fraction Variation with Temperature for Fuels

$\mathrm{V}-7$

$\mathrm{V}-8$

Oxidizer Tank Pressure Variation with Temperature . .

$\mathrm{VI}-1$

Fue1 Tank Pressure Variation with Temperature . . . .

Fruhof Low-g Surface Tension Propellant Acquisition

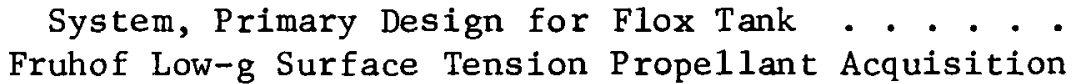

VI-2

System, Primary Design for MMH Tank . . . . . . . . .

VI-3

Fruhof Low-g Surface Tension Propellant Acquisition

System, Alternative Design for Flox Tank . . . . . .

VI-4

Fruhof Low-g Surface Tension Propellan, Acquisition

System, All-Welded Design for Flox Tank. . . . . . .

VI -5

VI-6

VI-7

VI-8

VI-9

VI-10

VI-11

VI-12

VI-13

Interface in Oxidizer Tank

. . . . .

.....

Standpipe with Vanes for Oxidizer Tank . . . . . . .

Interface in Fuel Tank . . . . . . . . . . . . . .

Standpipe with Vanes for Fuel Tank . . . . . . . . .

Oxidizer Tank Standpipe Volume .. . . . . . . . . .

Interfaces within Standpipe .. . . . . . . . . . .

Curvature vs Liquid Volume for Standpipe . . . . . . .

'Curvature vs Volume of Liquid within Vanes . . . . . .

Fue 1 Tank Standpipe Volume . . . . . . . . . . .

VI -14

Liquid Orientation at Various Points in Mission

V-21

V-26

V-27

$\mathrm{V}-30$

$\mathrm{V}-31$

$\mathrm{V}-34$

$\mathrm{V}-35$

$\mathrm{VI}-3$

VI-5

VI-7

VI-9

VI $\div 31$

VI -32

VI -34

VI -34

VI-36

VI -37

VI-39

VI -40

VI -42

VI -43 
Table

I-1 Trajectory Correction Propulsion Event Sequence . . . I-5

I-2 Acceleration Environment . . . . . . . . . . . I-6

I-3 Propulsion System Criteria . . . . . . . . . . . I-9

I-4 Criteria Matrix . . . . . . . . . . . . . . . . I I-14

III-1 Effect of Post Configuration on Interface Shape. . . . III-9

III-2 Volume of Liquid Outside Standpipe . ... . . . . . III-24

III-3 $\sigma / \mathrm{R}$ for Each Propellant . . . . . . . . . . . . . III-49

III-4 Value of $\omega^{2}$ for Each Propellant Tank . . . . . . . . III-57

III-5 Center of Gravity Coordinates . . . . . . . . . . III-61

III-6 Location of $\mathrm{cg}$, In-Line Tanks . . . . . . . . . . III-65

III-7 Location of $\mathrm{cg}$, Side-by-Side Tanks . . . . . . . . . III-65

III-8 Modified Trajectory Correction Propulsion Event Sched-

ule...................... III-78

III-9 Pressurization System Requirements for Nominal Propel-

lant Temperature Conditions... .. . . . . III-78

III-10 Tank Pressures at the End of Coast . . . . . . . . . III-84

III-11 Helium Usage, $1 b_{m}$. . . . . . . . . . . . . . III-84

III-12 Propellant Tank Temperature and Pressure Before and

After $17^{\circ} \mathrm{R}$ Increase in Equilibrium Temperature (60-

sec Pressurization ................ . III-85

III-13 Maximum Finger Thickness (in.) . . . . . . . . . III-110

IV-1 Drop Tower Tests . . . . . . . . . . . . . . IV IV-2

IV-2 Bond Numbers for Stability Tests . . . . . . . . . . IV-23

IV-3 Scaling Factors . . . . . . . . . . . . . . . . . IV-24

V-1 Evaluation Criteria Weighting Factors . . . . . . . . V V-6

V-2 Candidate Concept Rating Factors . . . . . . . . . . V V-7

V-3 Calculated Figures of Merit for Candidate Concepts . . V-14

V-4 Prope11ant Property Comparison .. . . . . . . . V-17

V-5 Interface Stability Index . . . . . . . . . . V V-18

V-6 Capillary Pumping Index, Nominal Temperature . . . . V-24

VI-1 Last Burn Volumes ................ . VI-48 
SUMMARY

The program was conducted to select and recommend for further development and performance verification the best surface tension propellant acquisition concept for an advanced spacecraft propulsion system having a 10-year mission capability. Surface tension systems were specified because they were shown to be the best propellant acquisition technique for various interplanetary spacecraft in a prior study (Contract NAS7-754). A variety of surface tension concepts for accomplishing propellant acquisition were formulated for the baseline Space Storable Propulsion Module and Jupiter Orbiter Mission. Analyses and evaluations were then conducted on each candidate concept to assess fabricability, performance capability, and spacecraft compatibility. A comparative evaluation of the results showed the Fruhof-class of $10 \mathrm{w}-\mathrm{g}$ surface tension systems to be preferred for these interplanetary applications. They can be adapted to a broad spectrum of low-g applications and can be tailored to specific applications with the desired operational margin. The evaluations also showed that the space-storable propellants present a much more difficult propellant acquisition situation than their earth-storable counterparts because of both propellant property differences and system fabrication considerations.

A preliminary design of the selected Fruhof standpipe with vanes system was accomplished based on analysis and testing of the more significant factors influencing its fabrication and operation. This system is preferred for further design study because it appears most capable of meeting spacecraft and mission requirements of the type evaluated. 
Controlled propellant orientation throughout long-duration (up to 10 years) interplanetary missions is a prerequisite for successful performance. The propellant acquisition subsystem must provide gas-free liquid on demand for both long- and short-duration engine firings of a multiburn mission having up to two years between burns. In addition, provisions for pressurization, emergency vapor venting, and center-of-gravity control are required.

Surface tension propellant acquisition subsystems are particularly well suited to these low-gravity, multiburn, long-1ife missions. This was shown in a previous study (Contract NAS7-754), which evaluated various types of propellant acquisition devices for advanced spacecraft applications and found surface tension systems to be superfor ( $\operatorname{Ref} I-1$ and $I-2$ ). Under the previous program, detailed analyses and designs of two different surface tension systems were then made for three reference missions. It was here that the Martin Marietta Fruhof low-gravity propellant acquisition concept was developed. This system was preferred, if testing under minus one $\mathrm{g}$ was not a requirement.

With a surface tension device established as the preferred approach, this Space Storable Propellant Acquisition System Program was the next step in the progression to provide an acquisition system for the Space Storable Propulsion Module. It was conducted by Martin Marietta Aerospace under the direction of the Jet Propulsion Laboratory to compare surface tension devices only, and to select the best surface tension system. The program is part of NASA's Advanced Technology Program to provide long-life propulsion systems for unmanned missions to Jupiter and the outer planets during the early 1980s. The effort described in this report comprises the first phase of a program for the development and comprehensive definition of a design for a surface tension propellant acquisition system for use in these advanced spacecraft propulsion systems. Specific program objectives were to:

1) Select and recommend for further development and performance verification the best surface tension propellant acquisition system for the Space Storable Propulsion Module and Jupiter orbiter mission, and,

2) Prepare a detailed design of the selected system for the baseline spacecraft and mission. 
B. MISSION AND SPACECRAFT CRITERIA

The baseline mission and spacecraft criteria used in the program are presented in this section. The information is divided into two parts which describe the mission and the propulsion system.

1. Mission Description

The baseline mission for evaluation and design of the space storable propellant acquisition concepts was that of the Jupiter Orbiter. This mission consists of four phases: ground hold; launch and injection; Jupiter transfer; and Jupiter orbit. These phases are discussed briefly in the following paragraphs.

a. Ground Hold - The time between propellant loading in the tanks (which nominally would occur at the same time as encapsulating the spacecraft in the shroud) and launch could be as long as 60 days. Therefore, active propellant temperature conditioning must be provided by the aerospace ground equipment/ground support equipment from the end of propellant loading until vehicle liftoff. $A L_{2}$ cooling line is provided in the oxidizer tanks to perform the conditioning process. After liftoff, the onboard thermal control system will provide propellant temperature control.

b. Launch and Injection - The spacecraft with an injected mass of $3150 \mathrm{lb}$, will be launched by a Titan IIID7/Centaur/Burner II. The ascent mode from launch to injection will use a parking orbit of $100 \mathrm{n} \mathrm{mi}$. Maximum coast time in the parking orbit will not exceed $1 \mathrm{hr}$.

c. Jupiter Transfer - During this portion of the mission, the spacecraft will be oxiented with the propulsion module on the shade side, except during reorientation for and during maneuvers. The maximum allowable time that the propulsion system may be in the sun will be 75 minutes. The Jupiter transfer phase will last 767 days with a sun-spacecraft orientation as shown in Fig. I-1.

d. Jupiter Orbit - The orbit insertion will result in a $1.44 \mathrm{x}$ $120 \mathrm{R}$ orbit, inclined to the Jupiter equator at an angle of 41.4 deg, with a period of 58 days. A plane change maneuver will be executed 797 days after launch to attain an equatorial orbit. During orbit, the spacecraft is to be Earth-oriented with the propulsion module on the shade side of the vehicle. During orbit trim maneuvers, the propulsion module may be randomly oriented and therefore may see the sun at some arbitrary angle. 


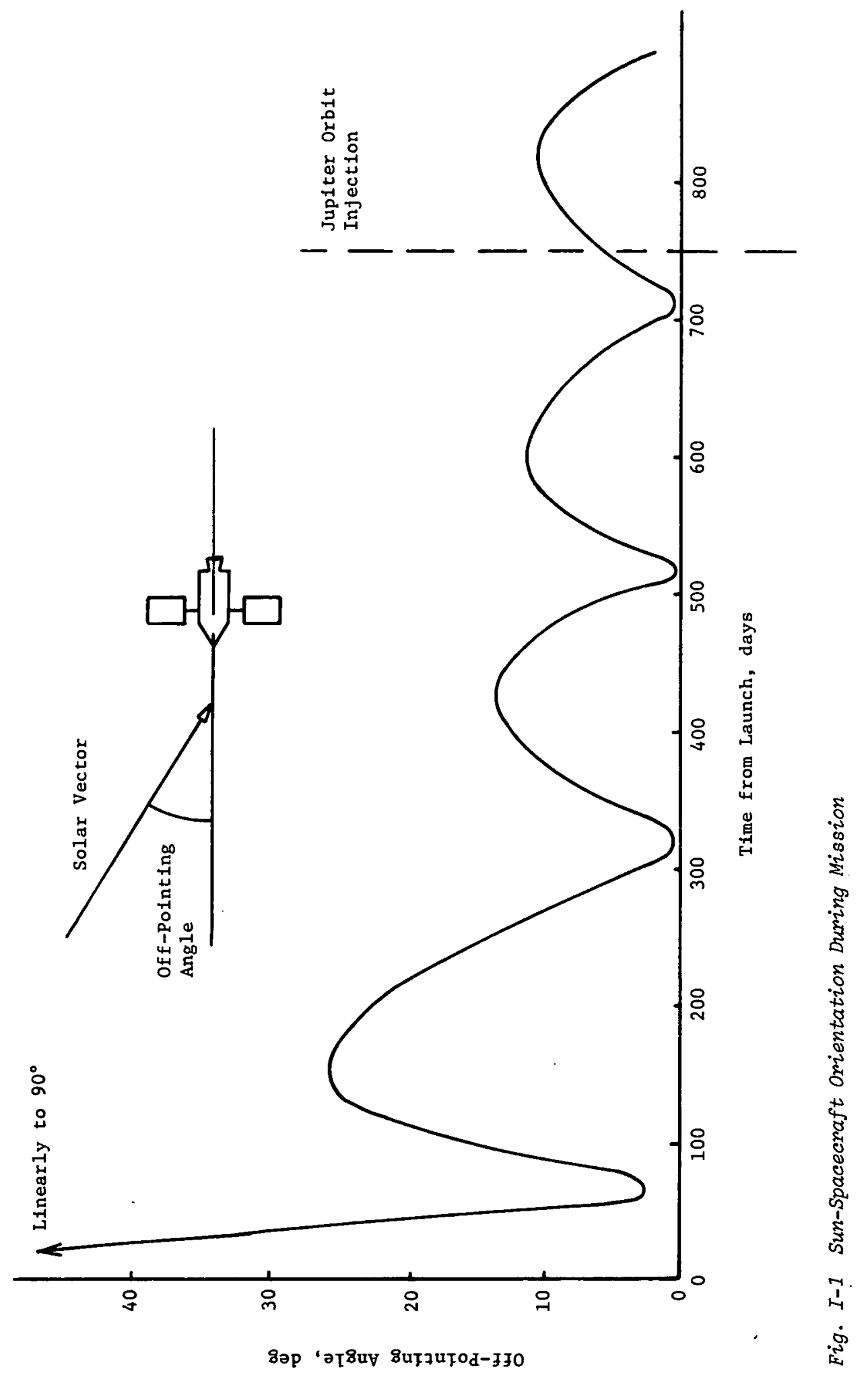


One objective of the mission is reconnaissance of Jupiter's Galilean satellites that are all in Jupiter's equatorial plane. Each satellite fly by is accomplished through a pre-encounter and postencounter maneuver that results in numerous orbit trims. The spacecraft will be occulted from the sun and Earth periodically, implying thermal and communication complexities with each Jupiter pass. Jupiter is surrounded by severe radiation and electromagnetic fields, intense enough to be a major consideration for spacecraft design and shielding.

The propulsion event sequence is shown in Table I-1. Twentyseven burns are identified as the nominal Jupiter Oribiter mission requirement. Two space storable propellant combinations were considered as baseline candidates for the propulsion system. These were the Flox $\left(88 \% \mathrm{~F}_{2}\right)$ and monomethyl hydrai ine $\left(\mathrm{CH}_{3} \mathrm{~N}_{2} \mathrm{H}_{3}\right.$ or $\mathrm{MMH}$ ) combination and the fluorine $\left(\mathrm{F}_{2}\right)$ and hydrazine $\left(\mathrm{N}_{2} \mathrm{H}_{4}\right)$ combination. Table $\mathrm{I}-1$ includes propellant mass requirements for each combination. An additional propellant combination, oxygen difluoride $\left(\mathrm{OF}_{2}\right)$ and diborane $\left(\mathrm{B}_{2} \mathrm{H}_{6}\right)$, was evaluated qualitatively to assess the impact this combination would have on the prope1lant acquisition system designed for the Flox/MMH system.

The propellant tank acceleration environment during the Jupiter mission is presented in Table I-2. Accelerations listed in the table were either given by or derived from the spacecraft criteria. Zero-g conditions are estimated to be on the order of $10^{-7}$ $g$, or less, arising from factors such as solar wind or planetary atmosphere drag. Engine burn accelerations are based on the total spacecraft mass and the thrust. Attitude control accelerations are based on the following criteria:

Maximum rotational velocity $=0.1 \mathrm{deg} / \mathrm{sec}$;

Maximum angular acceleration $=0.038 \mathrm{deg} / \mathrm{sec}^{2}$;

Maximum moment arm length - In-1ine tanks $=4.1 .4 \mathrm{in}$.

$$
\text { Side-by-side tanks }=18.0 \text { in. }
$$

The accelerations in the table were determined by a worst-case application of the above criteria. The accelerations produced by boom deployment are the sum of the rotational and translational components due to the torques applied to accelerate the deploying masses and the centripetal forces of the rotating masses. Spring and damping characteristics, the applicable dimensions, and the vehicle mass and inertia were used in the calculation (Ref I-3). 


\begin{tabular}{|c|c|c|c|c|c|c|c|c|c|c|c|c|c|c|c|c|}
\hline \multirow{4}{*}{\multicolumn{2}{|c|}{\begin{tabular}{|l} 
\\
\end{tabular}}} & \multirow{2}{*}{ 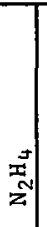 } & \multirow[b]{2}{*}{$\begin{array}{l}\infty \\
\dot{n}\end{array}$} & \multirow[b]{2}{*}{$\stackrel{m}{\stackrel{m}{\infty}}$} & \multirow[b]{2}{*}{ 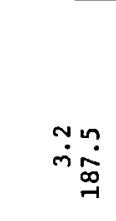 } & \multirow[b]{2}{*}{ ஸ் } & \multirow[b]{2}{*}{$\stackrel{\sim}{\sim} \underset{\dot{\infty}}{\stackrel{m}{\infty}}$} & \multirow{2}{*}{ 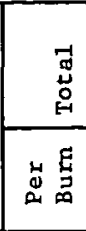 } & \multirow{2}{*}{$\frac{\dddot{8}}{\check{8}}$} & \multirow{2}{*}{ 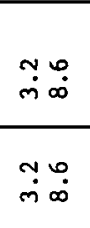 } & \multirow{2}{*}{$\frac{\ddot{m}}{\ddot{m}}$} & \multirow{2}{*}{ 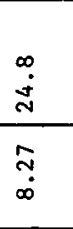 } & \multirow{2}{*}{ 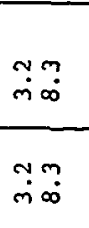 } & \multirow{2}{*}{ 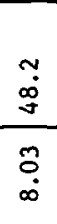 } & \multirow{2}{*}{ 묵 } & \multirow{2}{*}{$\underset{\infty}{0}$} \\
\hline & & & & & & & & & & & & & & & & \\
\hline & & \multirow[b]{2}{*}{ 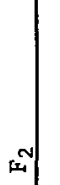 } & \multirow[b]{2}{*}{ l } & \multirow[b]{2}{*}{1} & \multirow[b]{2}{*}{$\underset{n}{\stackrel{n}{n}}$} & \multirow[b]{2}{*}{$\begin{array}{l}\stackrel{0}{\dot{J}} \\
\stackrel{+}{\circ}\end{array}$} & \multirow[b]{2}{*}{$\begin{array}{l}0 \\
\stackrel{n}{f}\end{array}$} & & 1 & İ & 1 & 1 & $\stackrel{0}{\circ}$ & 1 & \multirow{2}{*}{$\dot{\tilde{m}}$} & \multirow{2}{*}{$\stackrel{9}{\stackrel{9}{n}}$} \\
\hline & & & & & & & & 吕 & 1 & $\ddot{H}$ & I & $\mathbf{i}$ & $\stackrel{\circ}{\stackrel{0}{0}}$ & 1 & & \\
\hline \multirow{3}{*}{ 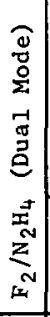 } & \multirow{2}{*}{\multicolumn{2}{|c|}{ 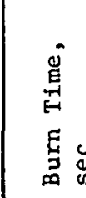 }} & \multirow[b]{2}{*}{ "n } & \multirow[b]{2}{*}{$\stackrel{n}{\mathfrak{g}}$} & \multirow[b]{2}{*}{ 品 } & \multirow[b]{2}{*}{ 부ำ } & & $\begin{array}{l}\text { 馬 } \\
\text { 总 }\end{array}$ & $\stackrel{m}{\dot{m}}$ & فㅁํㅁ & $\ddot{n}$ & 吕 & 递 & $\tilde{n}$ & 0 & $\checkmark$ \\
\hline & & & & & & & نำ & 岂苞 & $\begin{array}{l}\vec{\sigma} \\
\dot{\sigma}\end{array}$ & فㅁํ. & $\stackrel{\leftrightarrow}{\sim}$ & $\begin{array}{l}\hat{a} \\
\stackrel{\sim}{\sim}\end{array}$ & 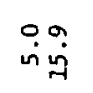 & & ڤึ & 㒸 \\
\hline & & 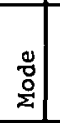 & $\stackrel{\circ}{0}$ & 芯 & 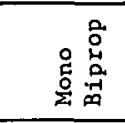 & 虽 & 号 & & 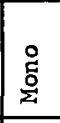 & 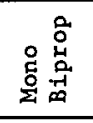 & 号 & 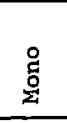 & 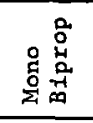 & 을 & & \\
\hline $\begin{array}{l}\widehat{\widehat{y}} \\
\frac{0}{2}\end{array}$ & $\AA^{a}$ & & & & & & & न & $\ddot{\circ}$ & $\stackrel{0}{r}$ & $\stackrel{\varphi}{0}$ & $\stackrel{n}{\mathfrak{j}}$ & $\stackrel{+}{r}$ & $\tilde{\infty}$ & & \\
\hline 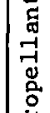 & 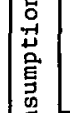 & 昰 & $\stackrel{0}{\dot{\sigma}}$ & $\vec{n}$ & ?ִ & $\stackrel{n}{i}$ & $\hat{n}$ & 岁㽞 & กิ & $\stackrel{\circ}{i}$ & $\stackrel{7}{\circ}$ & in & $\stackrel{\vec{r}}{r}$ & 年 & gु & : \\
\hline 商 & $\mid \begin{array}{l}5 \\
0 \\
\vec{u} \\
\vec{\sigma}\end{array}$ & & & & & & & 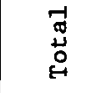 & & $\stackrel{0}{\dot{i}}$ & $\stackrel{n}{-}$ & 吕 & $\ddot{7}$ & 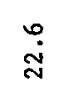 & $\Rightarrow$ & o? \\
\hline 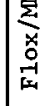 & 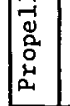 & $\frac{x}{0}$ & $\stackrel{-1}{\Im}$ & $\stackrel{\sim}{\sim}$ & $\stackrel{n}{\tilde{y}}$ & \begin{tabular}{l}
$\stackrel{0}{0}$ \\
$\dot{0}$ \\
\hdashline
\end{tabular} & 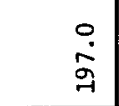 & 吕宫 & à & $\begin{array}{l}\dot{0} \\
\dot{\sim}\end{array}$ & $\stackrel{n}{m}$ & $\begin{array}{l}\infty \\
\dot{m}\end{array}$ & -1. & $\hat{i}$ & 官 & $\stackrel{\infty}{\text { m }}$ \\
\hline & $g$ & & & & & & & 点 & $\begin{array}{l}n \\
\tilde{n}\end{array}$ & $\ddot{\leftrightarrow}$ & $\dot{m}$ & $\stackrel{\infty}{0}$ & - & $\stackrel{\circ}{\circ}$ & 0 & 0 \\
\hline & 吉 & 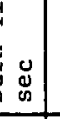 & $\ddot{0}$ & $\vec{j}$ & -1. & $\begin{array}{l}\overrightarrow{0} \\
\dot{\nu}\end{array}$ & $\underset{-1}{-1}$ & 出泀 & 弚 & $\stackrel{n}{0}$ & $\begin{array}{l}\text { N } \\
\stackrel{\sim}{0} \\
0\end{array}$ & 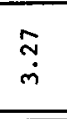 & $\ddot{0}$ & $\stackrel{m}{\tilde{m}}$ & $\dot{\infty}$ & $\stackrel{\circ}{\Omega}$ \\
\hline & 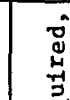 & 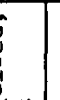 & & & & & & 宽 & ô. & $\begin{array}{l}n \\
0 \\
0\end{array}$ & $\begin{array}{l}\square \\
\dot{0}\end{array}$ & $\begin{array}{l}\tilde{m} \\
\dot{0}\end{array}$ & $\begin{array}{l}\text { nn } \\
\dot{0} \\
0\end{array}$ & $\stackrel{\circ}{\circ}$ & $\vec{b}$ & J \\
\hline & $\begin{array}{l}\mathscr{y} \\
\square\end{array}$ & $\frac{0}{0}$ & $\begin{array}{l}a \\
0 \\
0\end{array}$ & $\begin{array}{l}\vec{\Delta} \\
\dot{0}\end{array}$ & 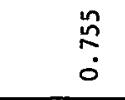 & $\stackrel{\stackrel{0}{\infty}}{\stackrel{0}{0}}$ & $\begin{array}{l}0 \\
\stackrel{8}{0} \\
: \\
0\end{array}$ & 岂 & $\begin{array}{l}-1 \\
0 \\
0\end{array}$ & $\stackrel{\leftrightarrow}{\circ}$ & $\overrightarrow{8}$ & $\begin{array}{l}\overrightarrow{0} \\
\dot{0}\end{array}$ & $\ddot{0}$ & $\ddot{0}$ & $\stackrel{0}{0}$ & - \\
\hline & 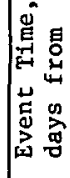 & & $n$ & 모 & $\stackrel{\circ}{\circ}$ & $\hat{\Omega}$ & $\underset{\infty}{\stackrel{\infty}{0}}$ & 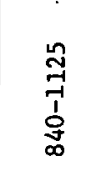 & & & & & & & & \\
\hline & & & & & & & & $\dot{z}$ & $n$ & $\rightarrow$ & $\Delta$ & $m$ & $\rightarrow$ & 0 & & \\
\hline & & $\begin{array}{l}\vec{g} \\
\dot{y} \\
:\end{array}$ & 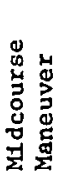 & 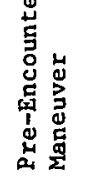 & 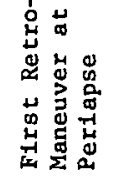 & 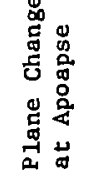 & 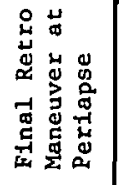 & 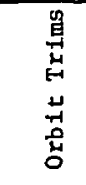 & $\begin{array}{l}\hat{1} \\
-1 \\
0 \\
\stackrel{0}{G} \\
\text { 品 }\end{array}$ & $\infty$ & సี & जे & $\stackrel{0}{\rightarrow}$ & $\underset{\tilde{I}}{\tilde{I}}$ & 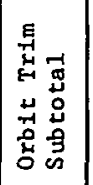 & 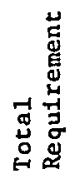 \\
\hline & E & $\dot{0}$. & -1 & $N$ & $m$ & $\checkmark$ & $n$ & & 7ี & $\tilde{H}$ & $\begin{array}{l}7 \\
\vdots \\
=\end{array}$ & $\begin{array}{c}\stackrel{0}{1} \\
0 \\
0\end{array}$ & $\vec{N}$ & 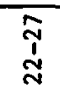 & & \\
\hline
\end{tabular}


Table I-2 Acceleration Environment

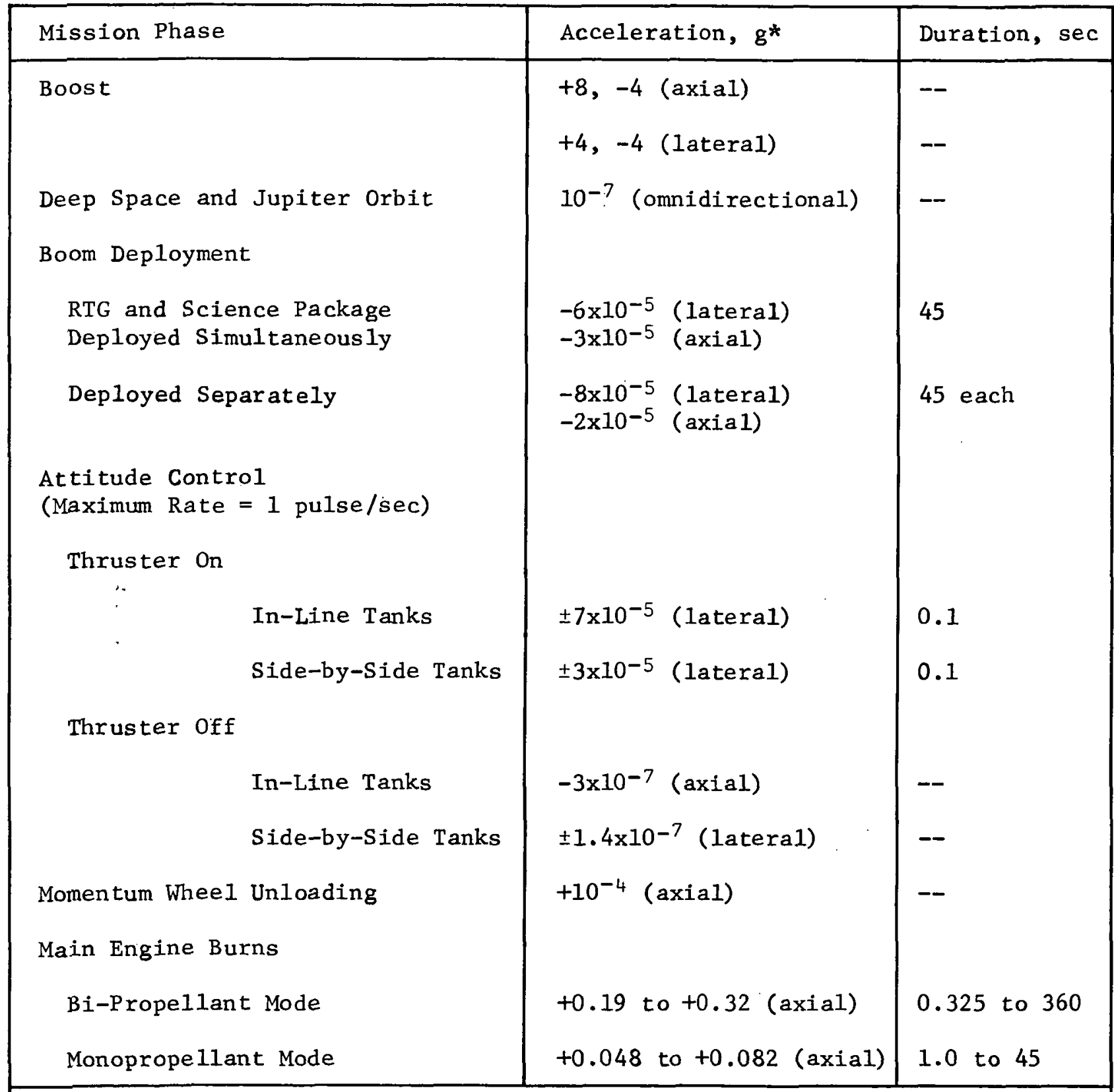

*A positive axial acceleration acts to settle propellants over the outlet. 
2. Propulsion System Description

The Space Storable Propulsion Module consists of three major assemblies: engine, pressurization, and propellant feed. The propellant acquisition systems are part of the propellant feed system. However, their design is affected by and also impacts on the operation of the other assemblies. A sketch of the propulsion module/spacecraft interface, derived from JPL Drawing 10041593, is shown in Chapter III (Fig. III-39). The propulsion system description is separated into two sections corresponding to the two candidate propellant combinations of primary interest.

a. Flow/MMH Propulsion System - The Flox/MMH system is shown schematically in Fig. I-2. Propellant tanks arranged in both a side-by-side and an in-line arrangement were considered to determine the effect, if any, on the propellant acquisition system.

The fuel pressurant and propellant tanks are mounted together on one side and wrapped together under a 0.3-in.-thick high-performance insulation blanket. The fuel tank is located so as to shade the oxidizer tank from the RTG. Further thermal isolation of the oxidizer subsystem is accomplished with the installation of a radiation shield between the two propellant tanks. On the oxidizer side, the pressurant tank is thermally coupled to the propellant tank and both tanks are insulated with 2-in.-thick polyurethane foam. These two tanks and their fluids are cooled during the ground hold operation by $\mathrm{LN}_{2}$ flowing in an internal coil in the oxidizer tank.

A summary of the pertinent Flox/MMH system criteria is presented in $\mathrm{Tab} l e \mathrm{I}-3$. Spherical tanks were the baseline configuration; however, any effects that cylindrical tankage may have on prope1lant acquisition system selection or operation were to be determined. 

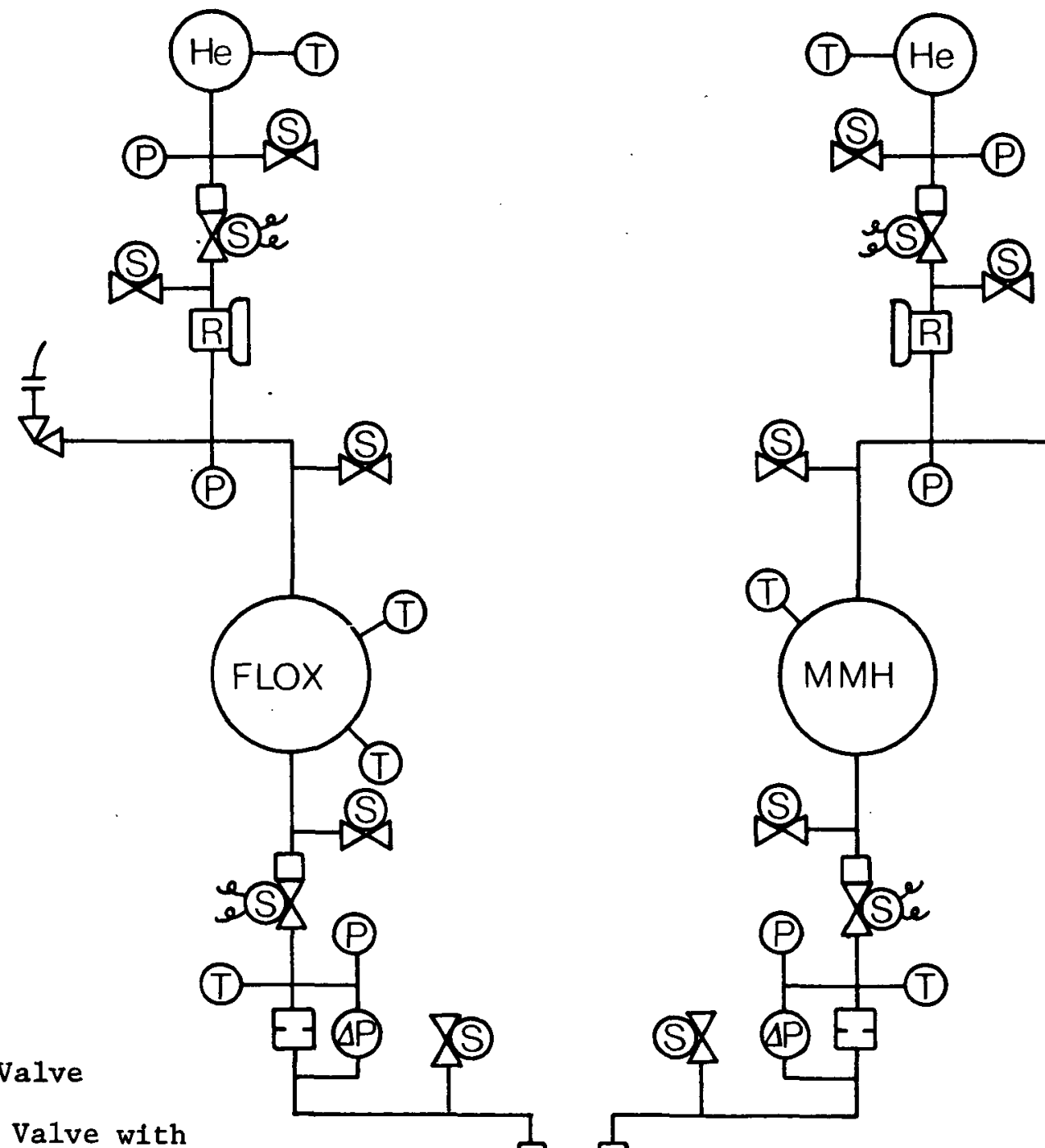

Service Valve

Solenold Valve with

Integral Filter

R Regulator

$E$ Trim Orifice

(1) Temperature

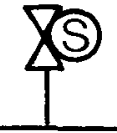

SX
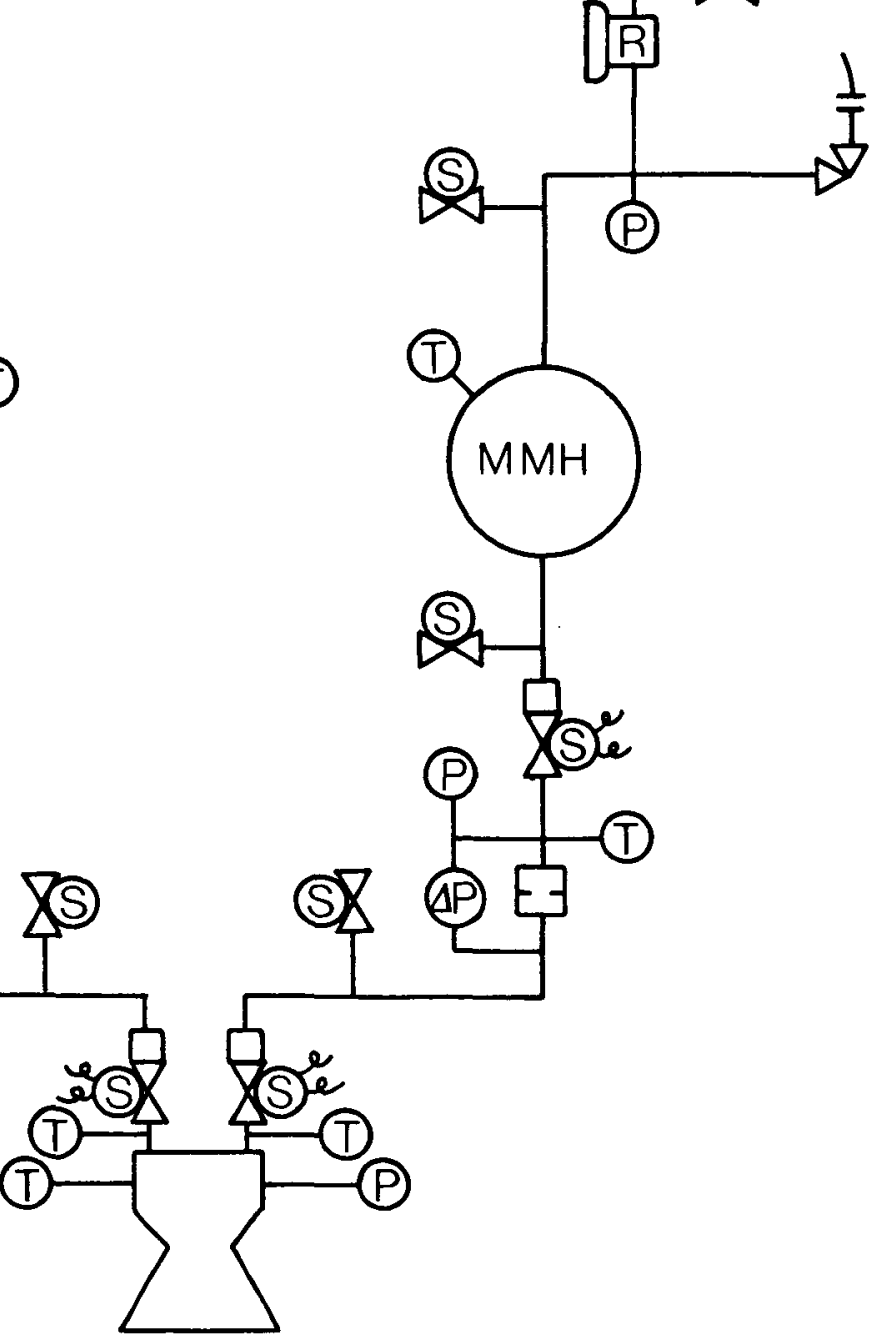

(1)

$\mathrm{MMH}$

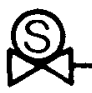

(P) Xste

(AP)

(1P) $\Delta \mathrm{P}$ Transducer

YH Rellef Device

Fig. I-2 FZox/MMH Propulsion Subsystem 
Table I-3 Propulsion System Criteria

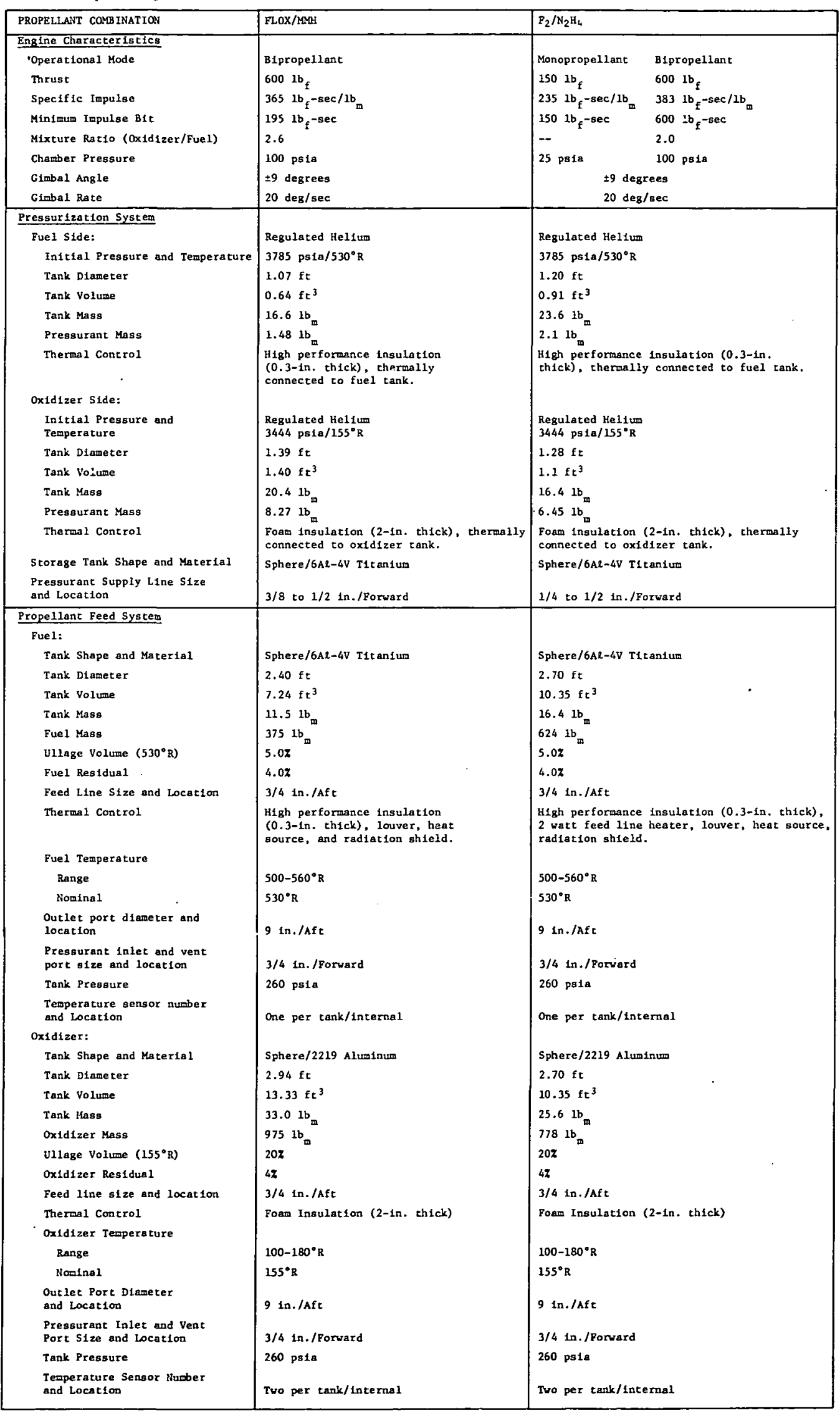


b. Dual Mode $\mathrm{F}_{2} / \mathrm{N}_{2} \mathrm{H}_{4}$ Propulsion System - The fluorine/hydrazine propulsion system, shown in Fig. I-3, is configured and thermally protected in a manner similar to the Flox/MMH system. However, it possesses a unique capability of operating in two engine modes, either as a 150-1b $\mathrm{f}_{\mathrm{f}}$ monopropellant engine or a $600-1 b_{f}$ bipropel-

lant engine. The engine is operated in the monopropellant mode for the small $\Delta \mathrm{V}$ maneuvers while the more efficient bipropellant mode is used for maneuvers requiring a $\Delta \mathrm{V}$ equal to or greater than $0.05 \mathrm{~km} / \mathrm{sec}$. However, even the bipropellant mode employs a 5-sec monopropellant start before initiation of $F_{2}$ injection. Another important feature unique to this system is the use of the hydrazine for a monopropellant attitude propulsion subsystem. Table I-3 also documents the $\mathrm{F}_{2} / \mathrm{N}_{2} \mathrm{H}_{4}$ system criteria.

C. STUDY GUIDELINES

Several guidelines were used in conducting the study. In formulating candidate surface tension concepts for accomplishing propellant acquisition, particular emphasis was to be given to advanced design concepts, such as those designed under Contract NAS7-754 (Ref I-2). Concepts that change or modify the tank internal geometry to position propellant were of primary interest, while devices using fine mesh screen were ruled out. However, the use of perforated plate was permissible. It was desirable that the acquisition device be adaptable to modular installation in the tank through a 9-in.-diameter access port. Also, cooling coils had to be integrated into the oxidizer system for groundhold propellant conditioning and the design had to accept temperature sensors in both the oxidizer and fuel tanks.

Several operational features were required. The primary requirement was that the propellant acquisition system be capable of supplying gas-free liquid to the engine throughout the mission. Control of the propellant center of gravity and limiting propellant slosh were also desired. Other considerations were: 


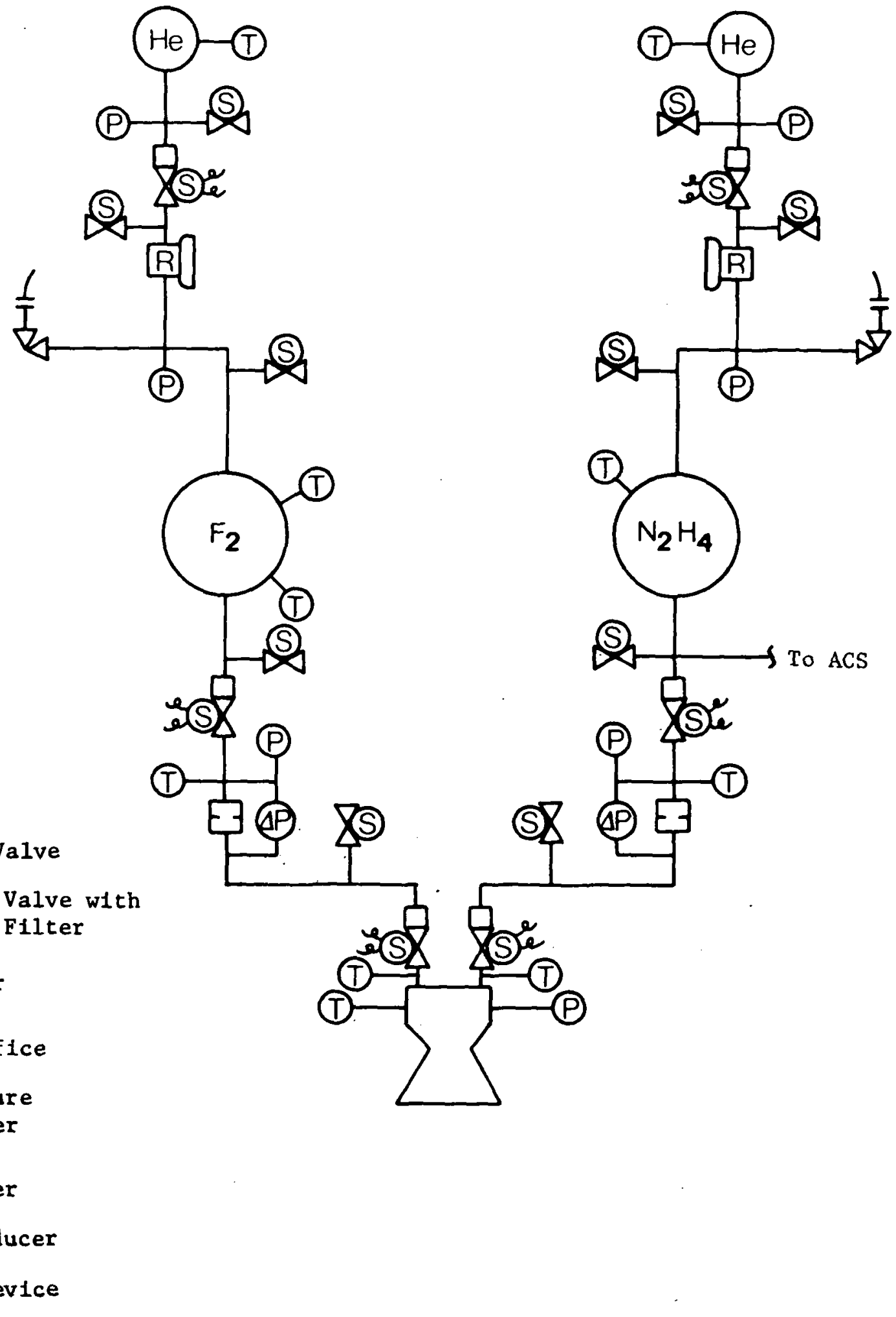

Figure I-3 $\mathrm{F}_{2} \mathrm{~N}_{2}{ }_{4}{ }_{4}$ Dual-Mode Propulsion Subsystem 
- The system had to perform and be compatible with space-storable propellants over a nominal mission life of ten-years;

An emergency pressure relief capability was desirable;

- Performance was not to be impaired by in-flight pressurization or thermal transients;

- The system was to be capable of off-design operation.

D. APPROACH

The program was conducted in four separate tasks, including documentation, as shown in Fig. I-4. An orientation meeting was held at JPL to initiate the program and the Project Work Plan (Ref I-4) was prepared. In Task I, a preliminary design study of surface tension propellant acquisition devices was conducted. The system and mission criteria to be used in the program were documented and a variety of candidate surface tension concepts for accomplishing propellant acquisition were formulated for evaluation. These candidate concepts are discussed in Chapter II. Analyses and evaluations were then conducted on each candidate concept to assess its fabricability, performance capability, and compatibility with the spacecraft. A critia matrix was generated that itemizes the propellant acquisition system operations from fabrication through flight and also notes the various aspects that must be considered. This matrix (Table I-4) was used in the design and analysis effort discussed in Chapter III. An experimental drop tower program, discussed in Chapter IV, was conducted to support, verify, and complement the analytical evaluation of candidate concepts.

Those candidate propellant acquisition concepts shown by the Task I design and analysis effort to be capable of satisfying the spacecraft and mission criteria were evaluated and compared in Task II. The criteria maxtrix was used to establish the rating system that was then used to determine the best candidate concept. A comparis on of the propellant combinations with respect to surface tension propellant acquisiton system operation was also made as part of Task II. Results of the Task II comparative evaluations are presented in Chapter V. 

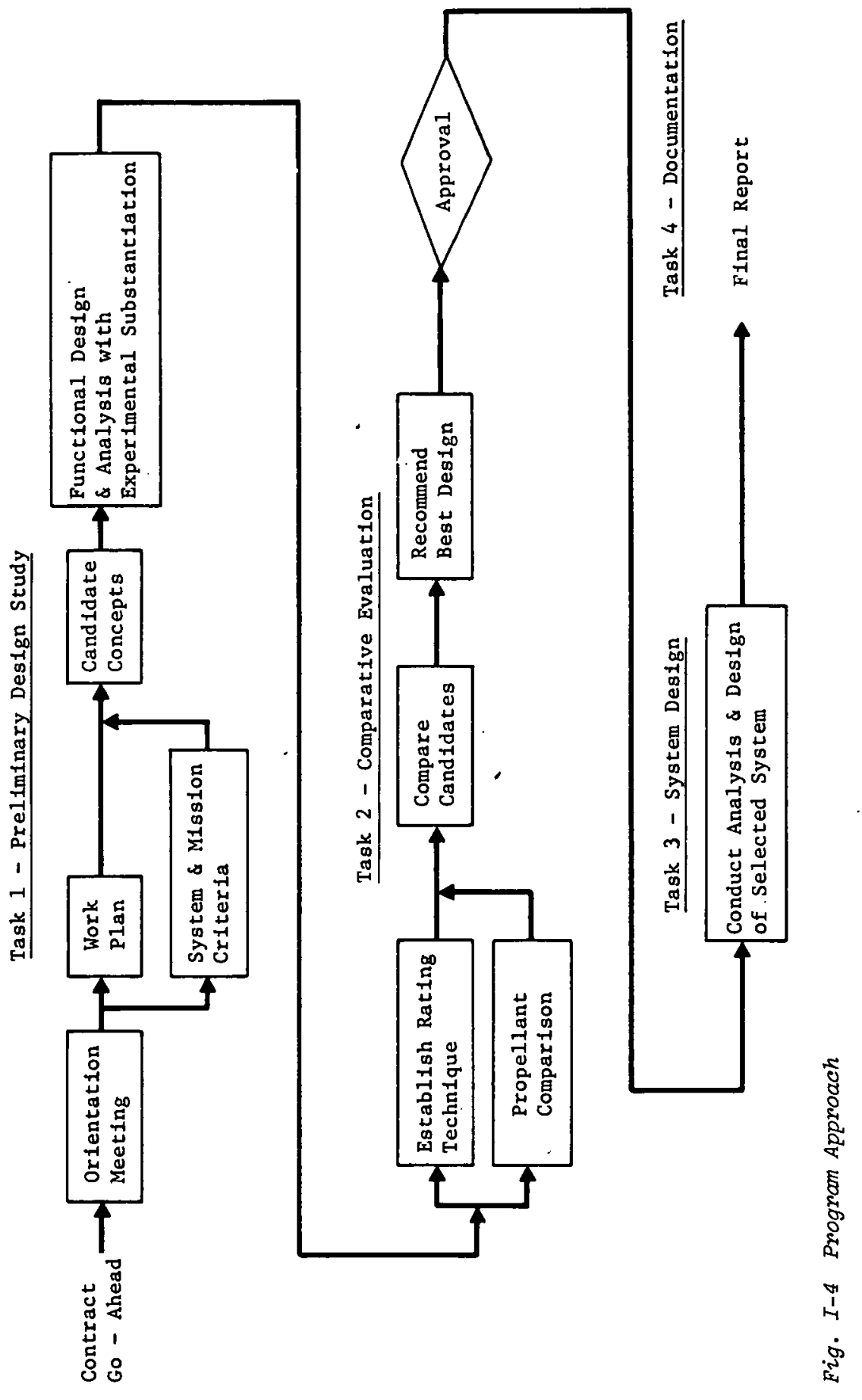


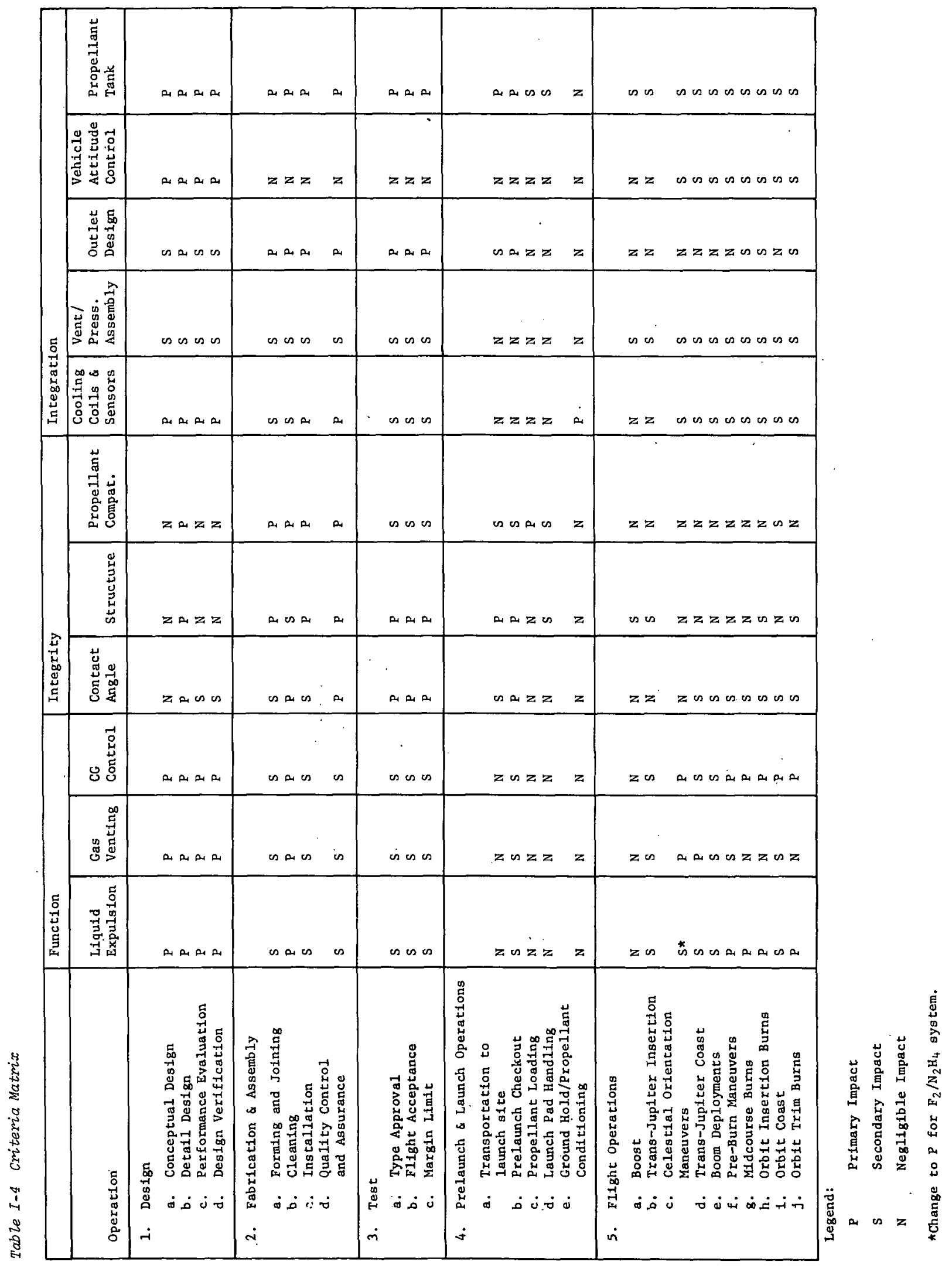


$\sigma$

Based on the comparative evaluation results, the best concept was recommended to JPL for approval. During Task III, detailed analyses and designs were made of the recommended and approved surface tension concept. The design is presented in Chapter VI. Finally, conclusions and recommendations are discussed in Chapter VII and references are listed in Chapter VIII. The recommendations in Chapter VII outline a plan for further development of the propellant acquisition system. 
A number of devices that appeared capable of functioning were conceived to initiate the process of selecting the candidate surface tension propellant acquisition concepts. In formulating these candidate devices, the effort concentrated primarily on concepts which modify the tank internal geometry to accomplish propellant positioning, since this approach was shown to be preferable for simflar applications in an earlier study (Ref II-1). Devices using fine mesh screen were not considered; however, no other restrictions were placed on the concepts, so that creativity would not be hindered. Any promising concept was added to the list of potential candidates.

After establishing the design requirements and mission parameters, a preliminary screening of the candidate concepts was conducted. Concepts appearing both practical and capable of meeting the spacecraft and mission requirements were selected as candidate concepts for further evaluation. Each of the selected candidate concepts fell into one of three general categories, based upon its configuration. These categories were:

A. Devices consisting of capillary barriers, which will retain liquid;

B. Devices contacting both the bottom and top of the tank, which will orient the liquid;

C. Devices located in the bottom of the tank, which will orient the liquid.

Category A devices retain liquid by using capillary barriers (Ref II-1). Some Category A devices are shown in Fig. II-1. The trap is the most basic of the Category A devices, consisting of a coverplate and a liner, as shown in Fig. II-1(a). Holes through the coverplate are small enough to retain liquid within the trap against adverse accelerations that tend to remove the liquid. Communication between liquid below the coverplate and the tank outlet is maintained by the annulus formed between the liner and the tank wall. The trap maintains a sufficient volume of liquid over the outlet so that the engine can always be started. Once enough propellant has been settled so that liquid flow to the engine can be maintained, the trap has accomplished its function. 


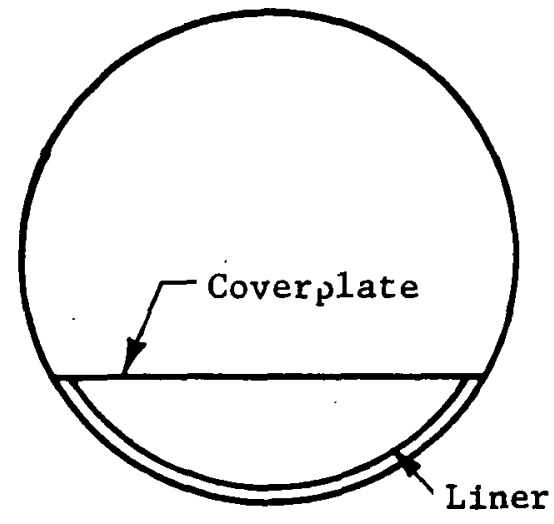

(a) Trap

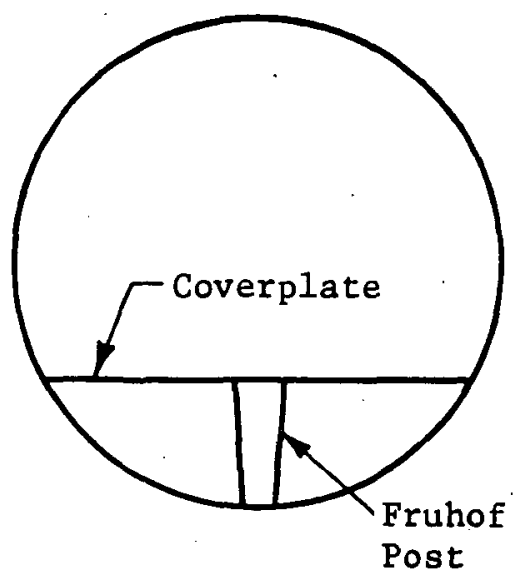

(b) Trap w1th Fruhof Post

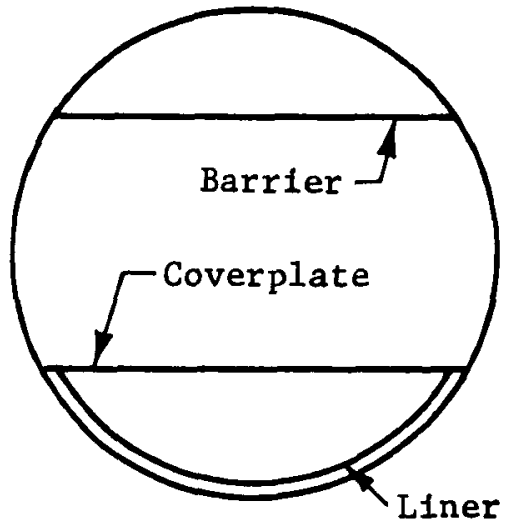

(c) Compartmented Tank

Fig. II-1 Category A Devices

It has been shown (Ref II-2) that fine mesh screen can be cleaned and passivated for use in fluorine, the most reactive of the propellants being considered. Compatibility was demonstrated for a period of 35 days and extrapolation to one year showed suitable performance should be obtalned; but, long-term compatibility (10 years) has yet to be substantiated. Because of this 10-year life requirement, perforated plate with a regular pattern of holes on the order of 0.1 to 1 in. diameter was considered for the capillary barriers. Micronic-sized holes were not considered because of potential problems with cleaning, clogging, etc. With the hole sizes under consideration, the device could not be tested in one$g$; it could operate only under the low-g environment of the mission. An advantage of using the larger hole sizes was that the trap could be designed to refill during the longer-duration spacecraft engine burns.

Two other variations that could be applied to the Category A devices are also shown in Fig. II-1. A Fruhof post could replace the liner and capillary barriers could be added at other levels in the tank to provide better control of the ullage bubble location. 
Both Category $B$ and $C$ devices make use of low-g fluid mechanics as an essential aspect of their operation. When the forces due to accelerations acting on a spacecraft are small in comparison to the capillary forces, surface tension and contact angle become the dominant factors that determine liquid position within a tank. In zero $g$, the equilibrium shape of the liquid-gas interface is established when its curvature is the same at every point on the surface. The Category $B$ and $C$ devices are designed so that the uniform curvature condition results in a reservoir of liquid 10cated over the tank outlet. The operation of the devices will be discussed in more detall in subsequent sections of this report.

Category B devices extend from the bottom to the top of the tank. Figure II-2(a) depicts the typical Category B device, the cruciform. The taper and the cruciform cross section tend to orient most of the liquid toward the outlet. Liquid located at the opposite end of the tank can flow along the cruciform to the outlet; the device itself provides communication. The split cruciform, shown in Fig. II-2(b), allows a gas bubble to be located symmetrically between the arms of the device. Another variation is illustrated in Fig. II-2(c).

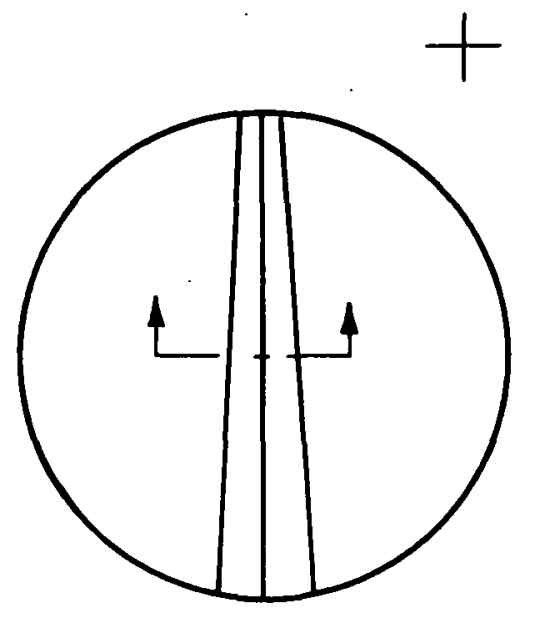

(a) Cruciform

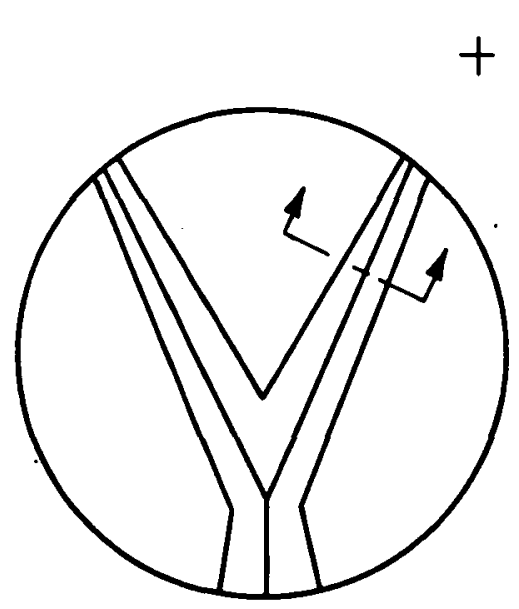

(b) Split Cruciform

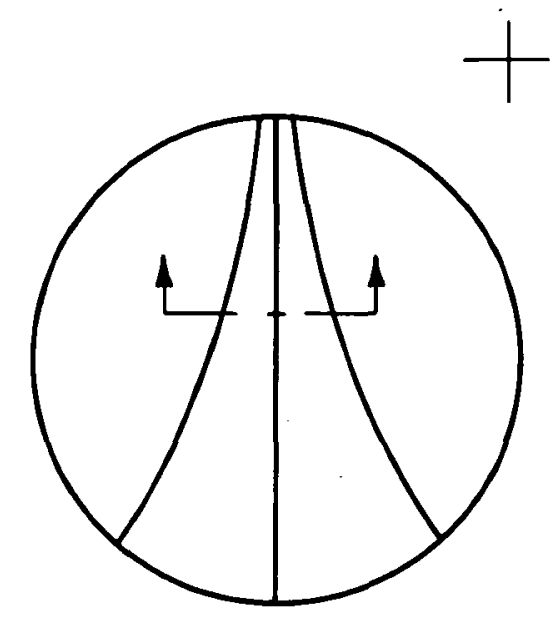

(c) Flared Cruciform

Fig. II-2 Category B Devices 
C. CATEGORY C DEVICES

Category $C$ devices function similarly to those in Category $B$. The primary difference is that the Category $C$ device is only in contact with the outlet end of the tank. These devices are all essentially derivatives of the Fruhof propellant acquisition system developed by Martin Marietta for JPL under Contract NAS7-754 (Ref II-1). The basic Fruhof post, shown in Fig. II-3(a), is the simplest of this type of device. Changes to the taper, size, and cross-section geometry are possible variations in the basic concept.

To minimize the volume of the device, the larger Fruhof posts would be hollow. These larger posts are referred to as "standpipes." Under low-g conditions, the internal volume of the standpipe will fill with liquid to provide a reservoir at the tank outlet.* Possible variations are different tapers and two or three concentric standpipes as shown in Fig. II-3(b).

A number of thin posts called "fingers" may be arranged off-axis to position the ullage bubble away from the outlet. The number and arrangement of the posts are variations. Some possibilities are shown in Fig. II-3(c).

By adding vanes to the post, improved interface stability and ullage positioning capability are obtained. The number of vanes and their profile are the variables considered. Figure II-3(d) illustrates some of these variations.

In addition to the four basic types of Category $C$ devices shown in Fig. II-3, there are a number of candidate devices that are combinations of the basic types. Some of the more attractive possibilities are illustrated in Fig. II 4 .

Because small amounts of liquid located at the end of the tank opposite the outlet may not be in contact with a Category C device, a means of providing communication must be added. This communication has not been shown in the sketches of the devices; however, it would consist of some form of channel mounted on the tank wall so as not to compromise the ullage centering capability. The specific configuration and number of channels required would depend on the mission parameters and the device.

*The radii of the standpipes under consideration are small in comparison to the tank radius so that the capillary pressure will tend to fill, rather than empty, the standpipe.

II-4 

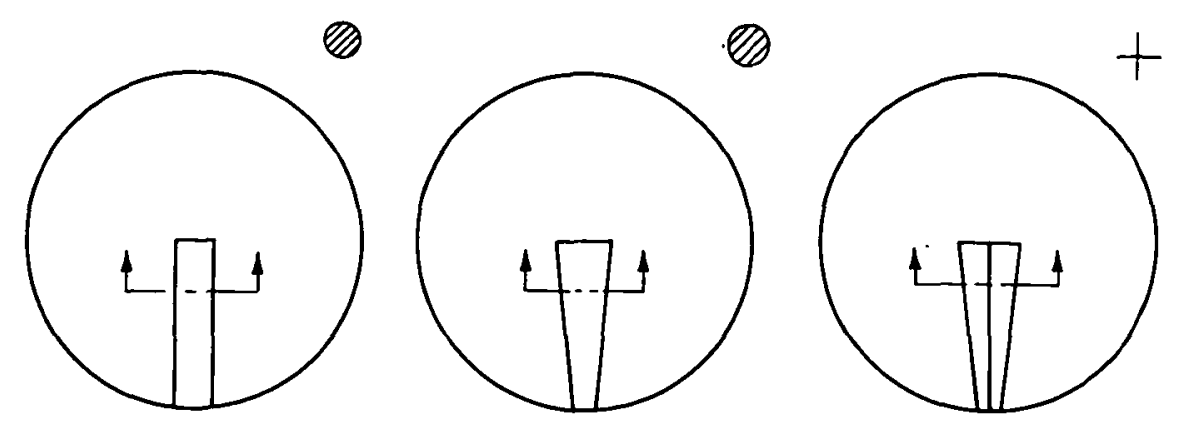

(a) Post

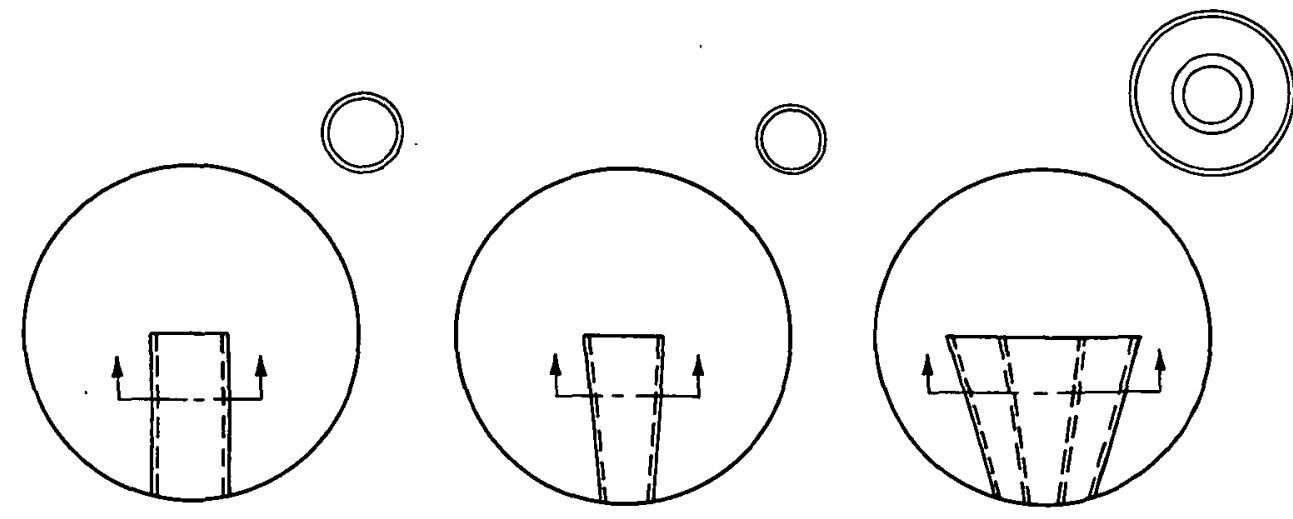

(b) Standpipe
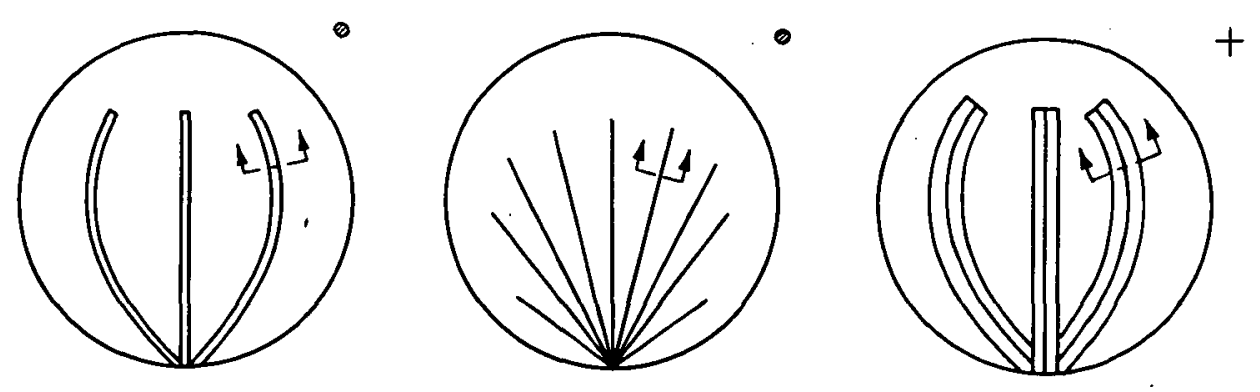

(c) Fingers
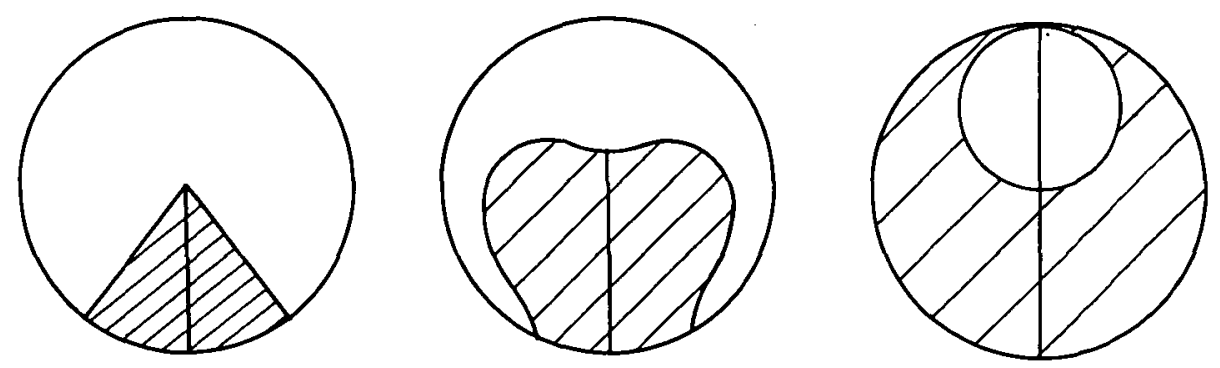

(d) Vanes

Fig. II-3 Category C Devices 


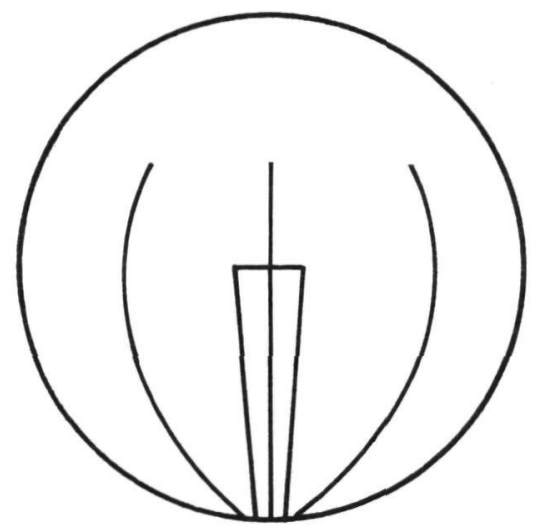

(a) Post with Fingers

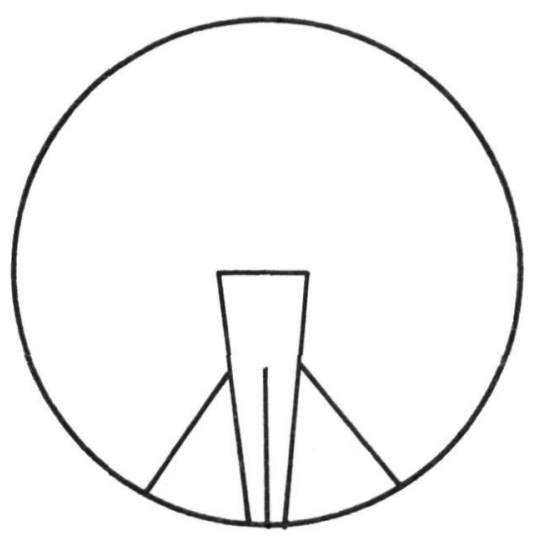

(b) Post with Small Vanes

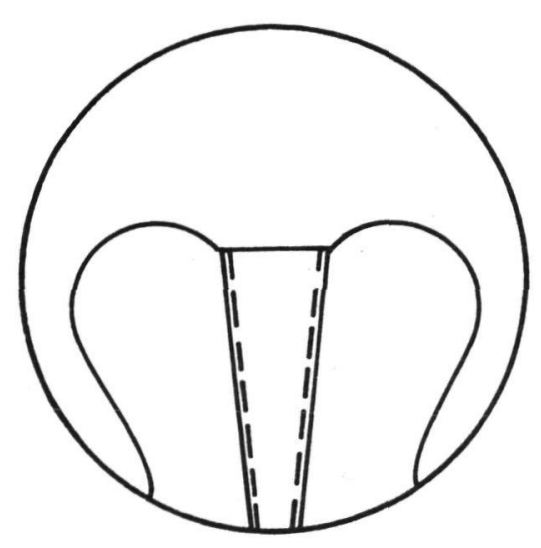

(c) Standpipe with Vanes

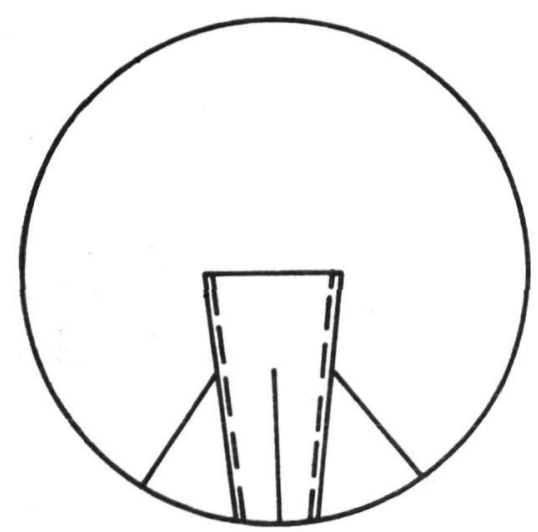

(d) Standpipe with Small Vanes

Fig. II-4 Category C Device Combinations 
Rather than analyzing all of the previously discussed candidate devices, a preliminary screening was accomplished. The objective of the screening was to select either the most representative device(s) in each category, or the device(s) appearing to offer the most promise for meeting the spacecraft and mission requirements. As a result of the preliminary screening, the devices shown in Fig. II-5 were selected as candidates.

of the Category A devices, the compartmented tank has the most advantages. An ullage region is formed by the additional barrier, through which venting and pressurization can be accomplished. The cruciform was selected as the candidate in Category $B$ due to its simplicity. It is the easiest to fabricate and install in the tank as a modular unit.

Due to their varied modes of operation and expected performance differences, five Category $C$ devices were selected as candidates. The tapered post has more capability than the other post devices. The straight-walled standpipe is the simplest standpipe device to fabricate and evaluate. Concentric standpipes complicate the tank outlet and the potential improvement in performance is not considered worthwhile. The combination device, post with fingers, is considered to be more capable than any of the pure finger devices. Fingers by themselves do not have sufficient surface area to orient the liquid.

A vane device, with a heart-shaped profile was selected. This profile is the most effective in positioning the ullage bubble to vent the tank. A combination of the standpipe and the vanes is also considered an attractive device.

Conceptual designs of each of these devices were accomplished, as discussed in the following chapter. Each design was then analyzed to determine its capabilities and limitations. During the ana1ysis, screening of the concepts was continued. When a device was found not capable of satisfying the mission requirements, it was dropped as a candidate. 


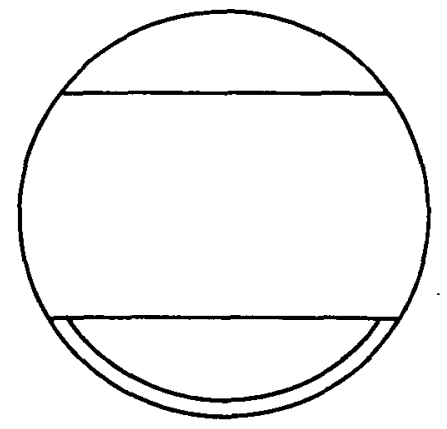

(a) Compartmented Tank Category A

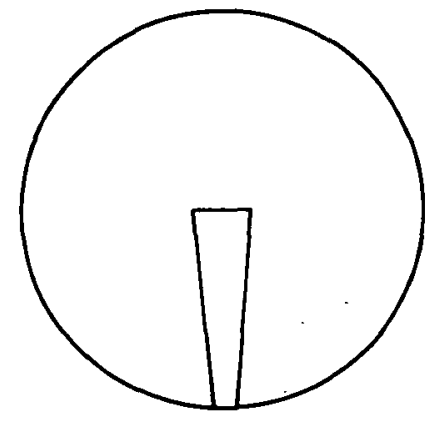

(c) Post

\section{Category C}

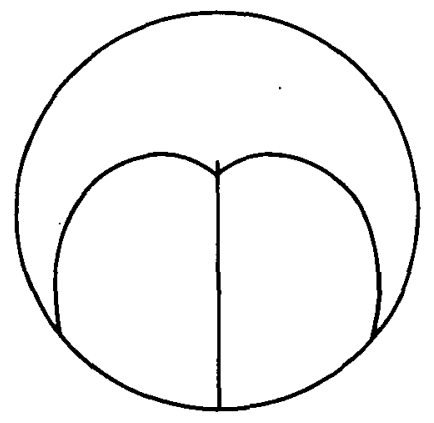

(f) Vanes

\section{Category C}

Fig. II-5 Candidate Devices

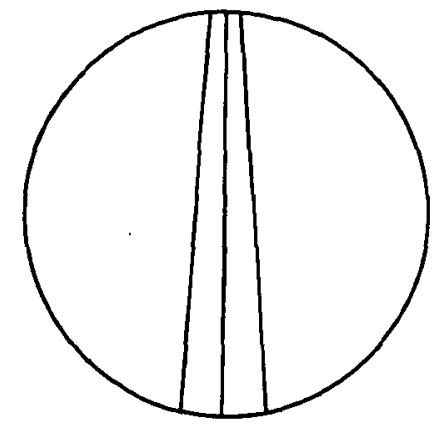

(b) Cruciform

Category B
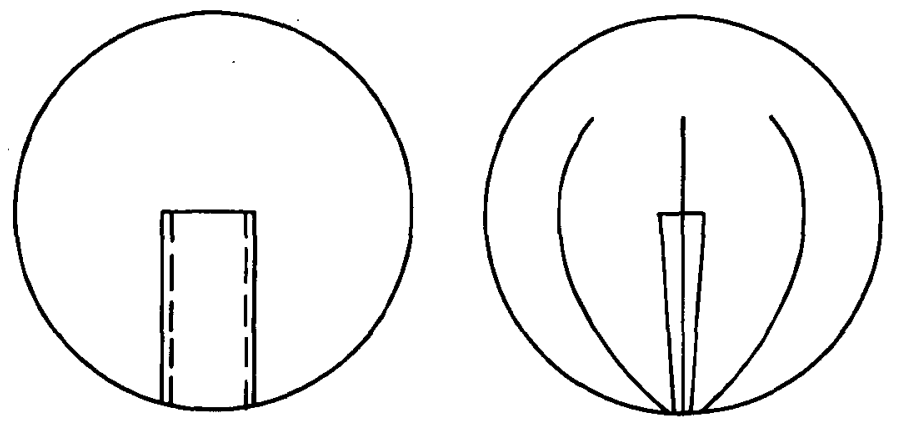

(d) Standpipe

(e) Post with Fingers

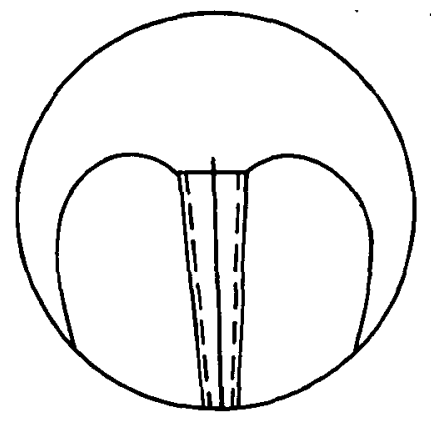

(g) Standpipe with Vanes 
All aspects recognized to be associated with or pertinent to the operation of the candidate surface tension propellant acquisition concepts are considered in this chapter. Included are analyses of system operations, interaction with the pressurization and thermal control subsystems, and discussion of system fabrication, assembly, and tank installation.

This portion of the analysis of the candidate propellant acquisition devices was concerned primarily with their actual operation during the mission. The function of primary importance is the ability of the device to provide gas-free liquid to the engine as required. Any factors or conditions that could affect this capability must be considered. Next in importance, the device must be able to provide for venting and pressurization, and control the center of gravity (cg) of the liquid.

Each of the candidate concepts is described and its operation discussed in this section. Following the discussions of each candidate, some of the more general analyses applying to most of the concepts are presented.

\section{Compartmented Tank}

The compartmented tank was selected as the best representative of the Category A devices (retain liquid by using capillary barriers). It consists of a trap plus additional barriers that subdivide the remainder of the tank into compartments.

a. Design - The trap maintains a reservoir of liquid at the :tank outlet, under all operating conditions, so that liquid will allways be available for engine start. As the engine starts, liquid outside the trap settles over the coverplate. Liquid can then feed from above the coverplate, through the trap, and out of the tank.

A coverplate retains liquid within the trap, and a liner forms an annular flow passage inside the trap to the tank outlet. The additional barriers provide some control of the gas and liquid located outside the trap. One additional barrier will be considered for this design. It is located so that liquid will remain below the barrier and gas above the barrier throughout the mission. This allows the tank to be vented and pressurized through the ullage above the barrier. A sketch of the device is shown in Fig. III-1. 


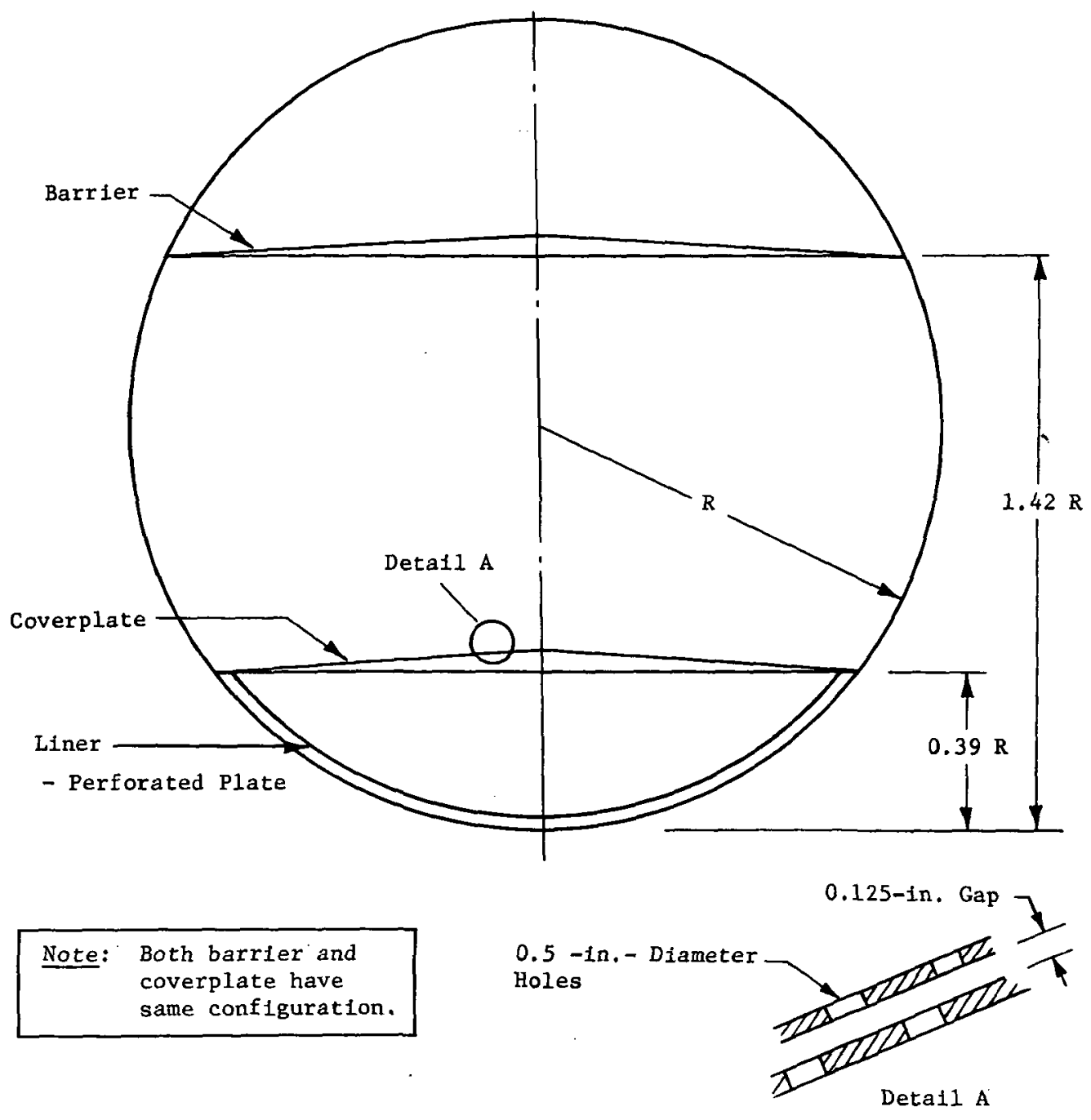

Fig. III-1 Compartmented Trap 
A propellant reservoir, with a volume equal to 10 percent of the tank volume, is formed over the outlet by the coverplate. The design approach was to size the trap so that all the propellant remaining after insertion into orbit will fit into the trap. The long-duration insertion burn will settle all the remaining propellant into the trap. With all the liquid in the trap at this point, only the motion of the liquid within the trap need be considered. This approach minimizes the problems of settling, suction dip, loss of liquid from the trap, etc, during the remaining short-duration burns.

The coverplate is conical and is constructed of perforated plate. Holes in the plate are $1 / 2$ in. in diameter. Two parallel plates with offset holes are used so that a completely wetted barrier can be maintained. If any part of the barrier should dry out, adverse accelerations will move liquid out of the reservoir until the barrier is rewet. A spacing between the perforated plates of $1 / 8$ in. will enable the gap between the plates to remain full of liquid, or refill if necessary, provided liquid is in contact with the barrier.

A liner, constructed of perforated plate, provides communication between liquid in the reservoir and the tank outlet. The size of the annular gap and the number and size of the holes in the liner are determined by analyzing the flow losses through the annulus to the outlet. Pressure drop due to flow through the liner and along the annulus, head losses, and the effect of the change in flow area are considered. A complete discussion of the design of the liner can be found in Reference III-1. The annulus formed by the liner must remain full of liquid. If liquid is lost from the annulus, gas ingestion is possible.

The additional barrier would be installed so that it would be at the height of the $1-g$ interface with the minimum initial ullage volume. Figure III-1 shows its placement for the initial $20 \%$ ullage of the oxidizer tanks. For the fuel tanks, the $5 \%$ initial ullage would require the barrier to be located at a height of 1.73R. This barrier would be similar in construction to the coverplate of the trap. Gas would initially fill the volume above the barrier and liquid would be retained below as long as the barrier remained wet.

b. Stability - Stability is based on the capillary pressure at a single pore in the perforated plate. A worst-case pore, at which the hydrostatic pressure is the greatest, is selected for the analysis. The stability of this type of device was previously investigated by Martin Marietta; the results can be found in 
Reference III-2. In this application, axial stability is the same as that for a cylinder, i.e., Bo $=0.84$ for a zero-degree contact angle. Lateral stability is defined by the $\phi$ number:

$\phi=\frac{\rho \operatorname{ahr}}{\sigma}$

where

$\rho=$ density,

$a=1$ ateral acceleration,

$r=$ radius of hole in perforated plate,

$\mathrm{h}=$ hydrostatic head,

$\sigma=$ surface tension.

A critical $\phi$ number of 2.0 establishes the stability limit for this condition.

The selected hole size for the perforated plate provides a safety factor of 2 , based on the diameter of the hole, for the worst-case application of the above stability design criteria. Because this device allows considerable overdesign, it can provide greater stability than the Category $\mathrm{B}$ or $\mathrm{C}$ devices.

Under the accelerations resulting from an engine burn, however, both the coverplate and the additional barrier will not be stable. The size of the holes are large enough to make the interface at the holes unstable during engine burn. Also the hydrostatic pressure, due to the slope of the barriers, exceeds the capillary pressure at the holes. During the longer-duration engine burns, gas in the trap will be purged and any liquid displaced from the trap, for whatever reason, will re-enter the reservoir. The operation of the additional barrier is similar. Any liquid that might have become located above the barrier would be forced out during the burn.

Cruciform

Category B devices extend from the bottom to the top of the tank and orient liquid over the outlet. The candidate device chosen from this category is the cruciform illustrated in Fig. III-2. This same device would be used in both the fuel and oxidizer tank. 


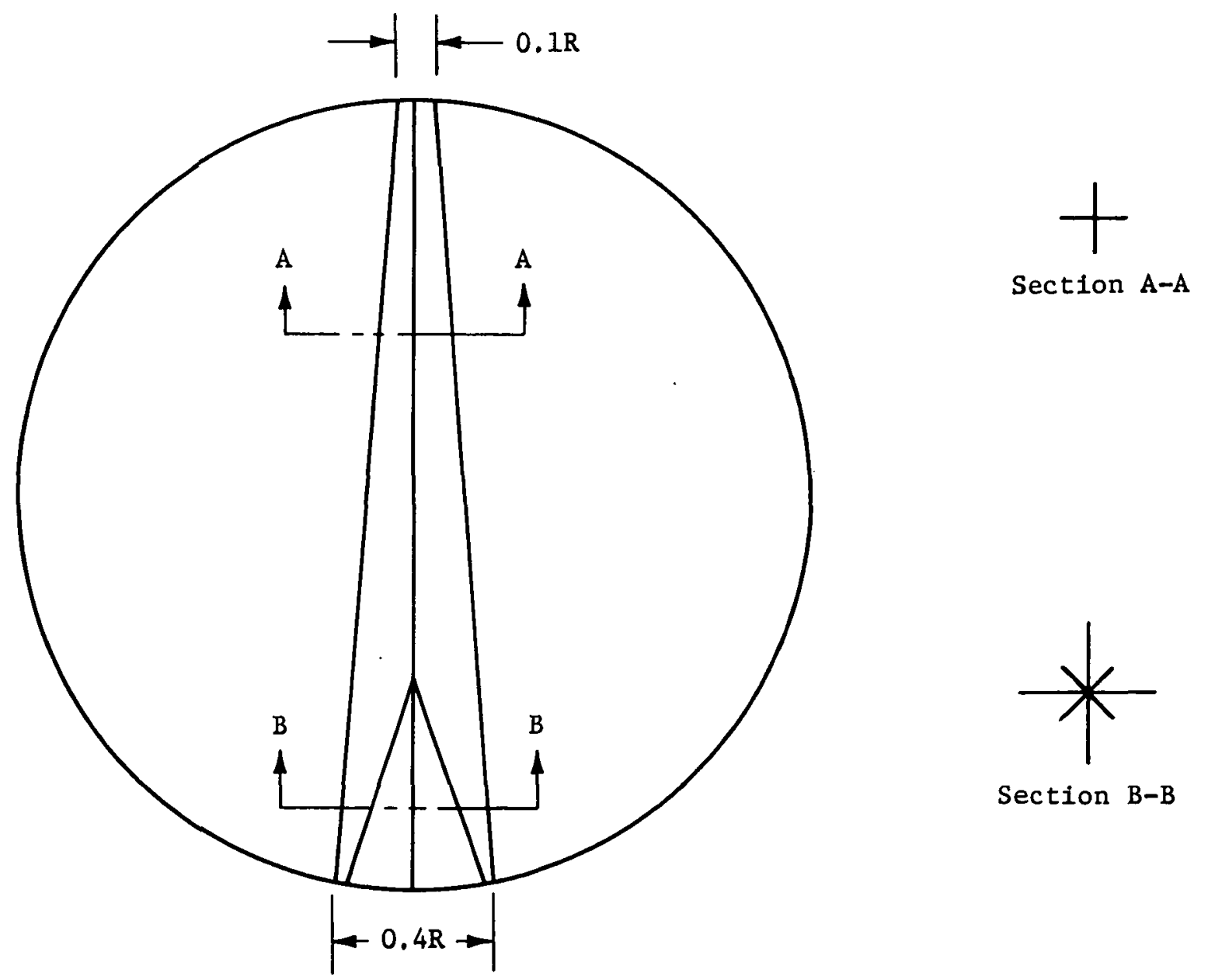

Fig. III-2 Cruciform

a. Design - The shape of the cruciform determines how the liquid will orient about the device in low $g$. A capillary pressure difference, which is proportional to the surface tension of the liquid and the curvature of the interface, exists across any interface. The magnitude of the pressure difference is given by the Young-Laplace equation:

$\Delta \mathrm{P}=\sigma\left(\frac{1}{\mathrm{R}_{1}}+\frac{1}{\mathrm{R}_{2}}\right)$ 
where

$$
\begin{aligned}
\Delta \mathrm{P} & =\text { capillary pressure differential, } \\
\sigma & =\text { surface tension, }
\end{aligned}
$$

$\mathrm{R}_{1}, \mathrm{R}_{2}=$ principal radii of curvature.

The two principal radii are defined for a given point, $A$, on the interface as shown in Fig. III-3. The centers of the arcs lie on a line, $A B$, perpendicular to the surface and passing through the point $A$ on the surface. $R_{1}$ and $R_{2}$ lie in planes that are perpendicular to one another and their intersection forms line $A B$. $\left(\frac{1}{R_{1}}+\frac{1}{R_{2}}\right)$ is referred to as the curvature. A flat interface has a curvature of zero and the more curved the surface (smaller radii of curvature), the higher the curvature.

In zero $g$, pressures within the liquid are determined solely by the Young-Laplace equation. Pressure equilibrium will be reached within the liquid and a static interface will be established when the curvature at every point on the surface is the same.

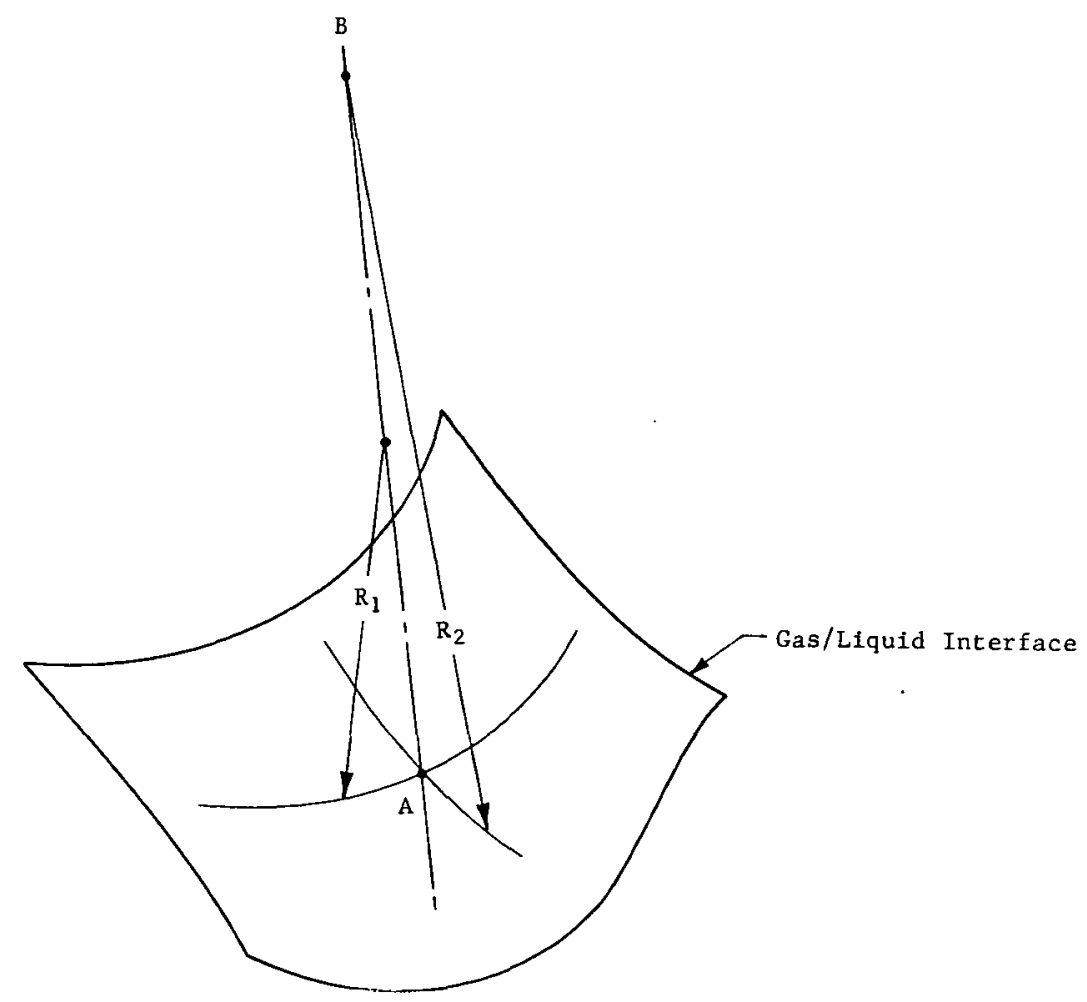

Fig. III-3 Principal Radii of Curvature 
The cruciform device takes advantage of the above described phenomena. To establish a static interface in zero $g$, the sharp corners of the device must fill until curvature can be equalized. The base of the cruciform is wider than the top, so the majority of the liquid collects over the outlet. A small cross section at the top. of the device allows only a small amount of liquid to collect at that location. Communication from the top of the tank to the outlet is provided along the device itself.

At low liquid volumes (less than $20 \%$ of the tank volume), liquid collects symmetrically about the cruciform. The interface about the cruciform was calculated for the Flox tank under zero $\mathrm{g}$ with the volume remaining after the final orbit insertion burn (Fig. III-4). It was assumed that the vanes of the cruciform were completely full of liquid, which is $31.2 \%$ of the total amount of liquid. Of the remainder, $64.2 \%$ of the liquid is at the outlet and $4.5 \%$ of the liquid is at the opposite end of the tank. In zero $g$ sufficient liquid is oriented over the outlet to permit start of the spacecraft engine. Once started, all the liquid will be settled so that a supply of propellant can be maintained for the duration of the engine burn.

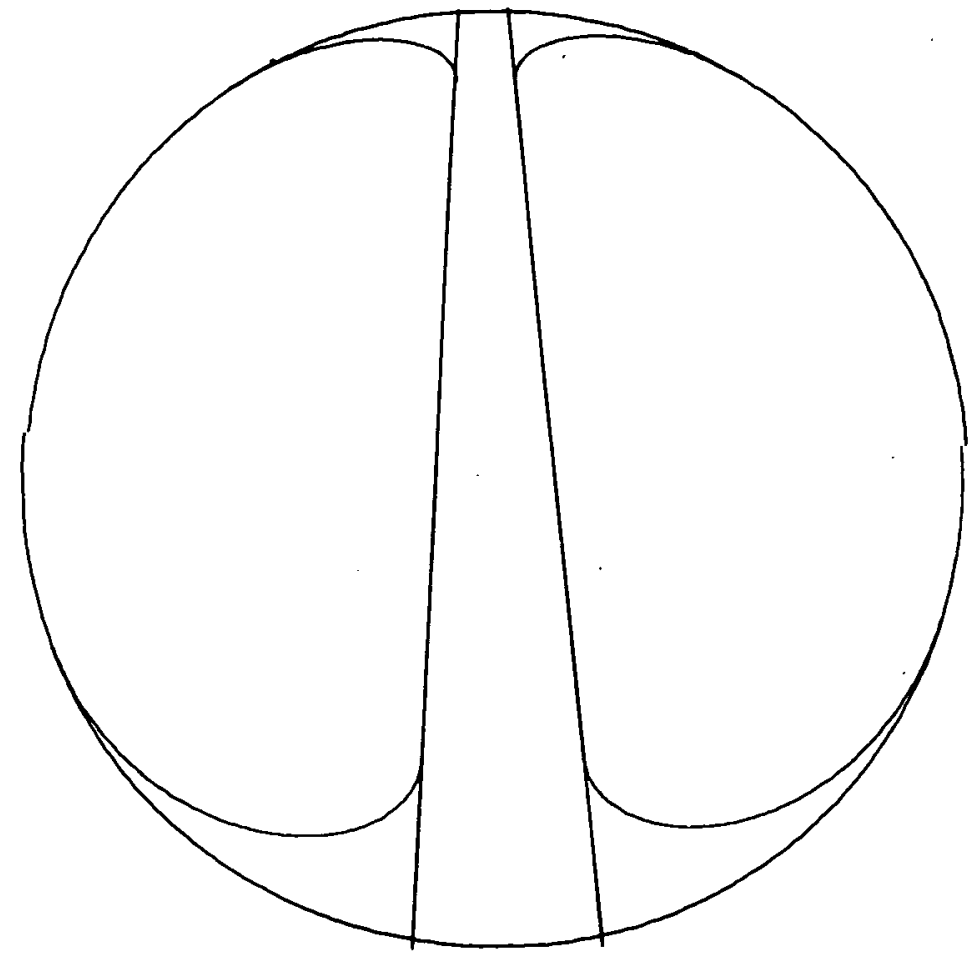

Fig. III-4 Zero-g Interface for the Cruciform 
For larger liquid volumes, the two interfaces at the top and bottom of the cruciform join to form a single interface whose shape is no longer clearly defined. One possible solution is an axisymmetric toroidal bubble of circular cross section; however, this is not the most stable configuration. A slight lateral disturbance will cause an offset, unsymmetrical kidney-shaped bubble to form. When this device was tested in the drop tower, the formation of the offset ullage bubble from the less stable toroidal bubble was demonstrated. Since the location of the ullage is indeterminate at the larger liquid volumes, neither venting nor pressurization could be reliably accomplished with this device.

The primary advantage of this category of devices is the good communication between the opposite ends of the tank. If liquid were displaced from the outlet, the large cross section of the device would readily return it. At the same time, this device has the disadvantage that a considerable portion of the liquid is held. within the vanes and at the top of the tank, rather than over the outlet (Fig. III-4). This liquid holdup about the device aggravates settling, slosh, and suction dip problems during engine start.

b. Stability - For this type of device, stability refers to the ability of the device to hold liquid about itself (over the tank outlet) under the effect of an adverse acceleration acting on the system. A stable liquid interface implies that no flow of liquid takes place under the applied acceleration environment. As the acceleration acting to move liquid away from the outlet is increased, however, a point will be reached at which a given volume of liquid will no longer be stable. Some liquid will flow away from the base of the device and a stable interface will be reestablished with a new, reduced liquid volume at the device base over the outlet. The axial and lateral stability of the device will be considered independently.

Some of the candidate devices allow an exact analysis of the axial stability limits to be performed. The analytical tools presently available do not allow an exact analysis of the stability of the cruciform, but its relative stability can be deduced. For any given axial acceleration the cruciform would hold a larger volume of liquid over the outlet than would the post or the post with fingers. However, it would be less stable than the vaned devices.

At the lower liquid volumes having a symmetrical interface, the relative stability of the cruciform to lateral accelerations would again fall between the post devices and the vane devices. At the larger liquid volumes, the offset ullage bubble has no stability. Any lateral disturbance will cause the bubble to be relocated to the side of the tank opposite the direction of the acceleration acting on the liquid. 
Due to their varied operation and performance, five Category $C$ devices (contact only the bottom of the tank over the outlet and orient liquid at this location) were selected as candidates. The simplest of these Fruhof-type devices is the post. The candidate designs for the oxidizer and fuel tanks are illustrated in Fig. III-5 and III-6, respectively. Geometrically, the device is an inverted, truncated cone located over the tank outlet.

Each of the Category $C$ devices includes a communication channel. Operation of the channel is included with the discussion of the standpipe with vanes.

a. Design - To select the diameter and taper of the posts, their effect on liquid orientation was evaluated for a single volume. Height of the post is arbitrary for this analysis. Before the last orbital trim burn, the volume of Flox is $0.312 \%$ of the tank volume. This volume was chosen because it is the smallest last burn volume fraction for both propellant combinations. The largest acceleration tending to move liquid away from the device was assumed to be acting on the tank.

The results for five different posts are compiled in Table III-1 and the interfaces are shown in Figure III-7. The point at which the interface contacts the post indicates how closely the liquid is held about the device. To provide a reference, the interface in a bare tank was calculated. The liquid is positioned in a thin layer at the end of the tank opposite the outlet. A post will hold the liquid in position over the outlet. As the diameter of the post increases, the liquid moves closer to the post; the same effect is achieved by increasing the taper. But, increasing the size of the post has the disadvantage of moving the liquid farther away from the centerline of the tank and decreasing the volumetric efficiency of the system. Configuration 4, selected for the post, is a good compromise between volumetric efficiency and retention capability.

Table III-1 Effect of Post Configuration on Interface Shape

\begin{tabular}{|c|c|c|c|c|c|}
\hline \multirow[b]{2}{*}{ Configuration } & \multicolumn{2}{|c|}{ Post Diameter Ratio, $\frac{\mathrm{d}}{\mathrm{R}}$} & \multirow{2}{*}{$\begin{array}{l}\text { Taper, } \\
\text { deg }\end{array}$} & \multicolumn{2}{|c|}{ Liquid Contact Point Ratio, $\frac{h}{R}$} \\
\hline & Top & Bottom & & Post & Tank Wall \\
\hline Bare Tank & -- & -- & -- & $\begin{array}{l}0.012 \\
\text { (at centerline) }\end{array}$ & 0.487 \\
\hline 1 & 0.1 & 0.1 & 0 & 0.133 & 0.159 \\
\hline 2 & 0.2 & 0.2 & 0 & 0.167 & 0.099 \\
\hline 3 & 0.1 & 0.05 & 1.73 & 0.100 & 0.264 \\
\hline 4 & 0.2 & 0.1 & 3.45 & 0.145 & 0.139 \\
\hline 5 & 0.3 & 0.1 & 6.87 & 0.156 & 0.121 \\
\hline
\end{tabular}




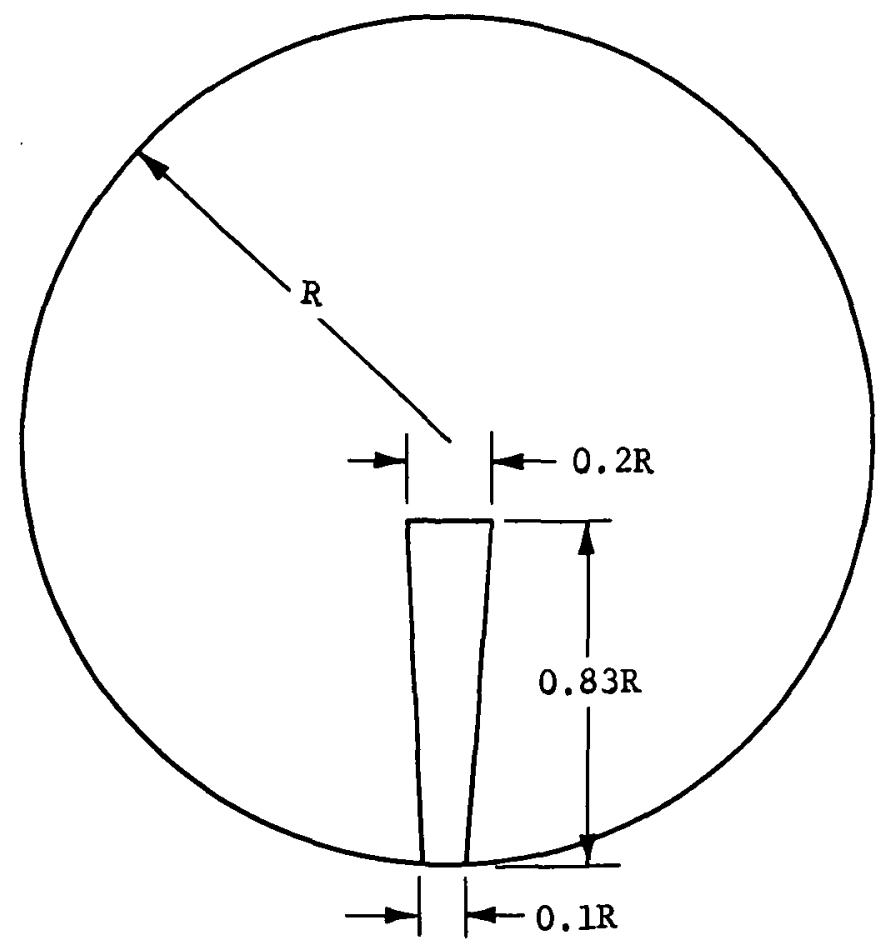

Fig. III-5 Post for Oxidizer Tank

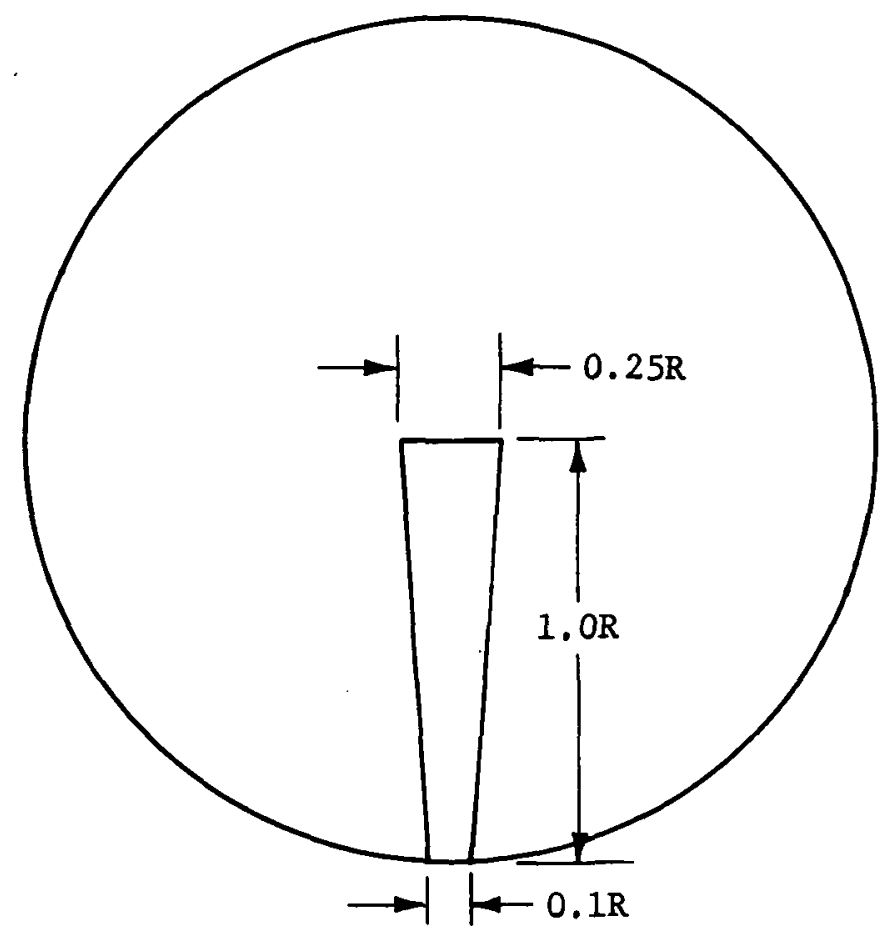

Fig. III-6 Post for Fuel Tank

III -10 


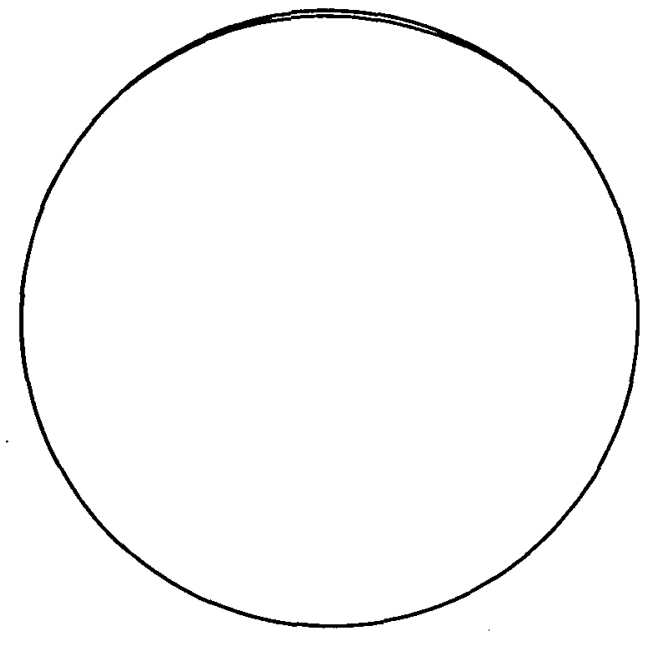

(a) Low-g Interface Shape, Bare Tank

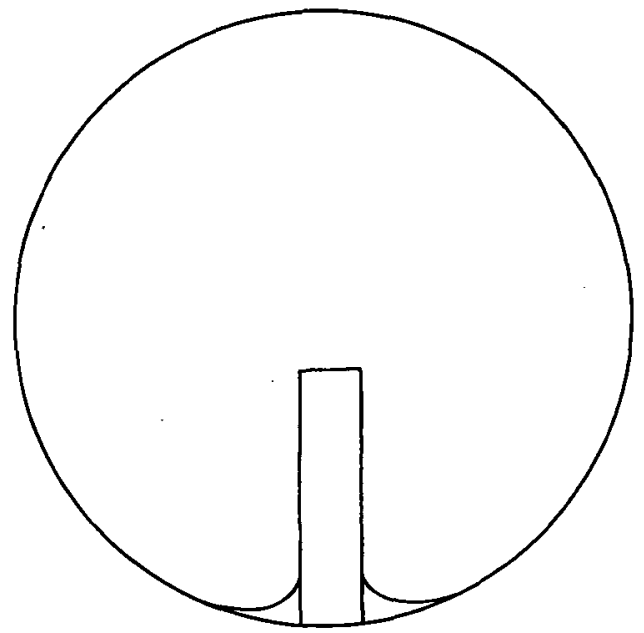

(c) Configuration 2

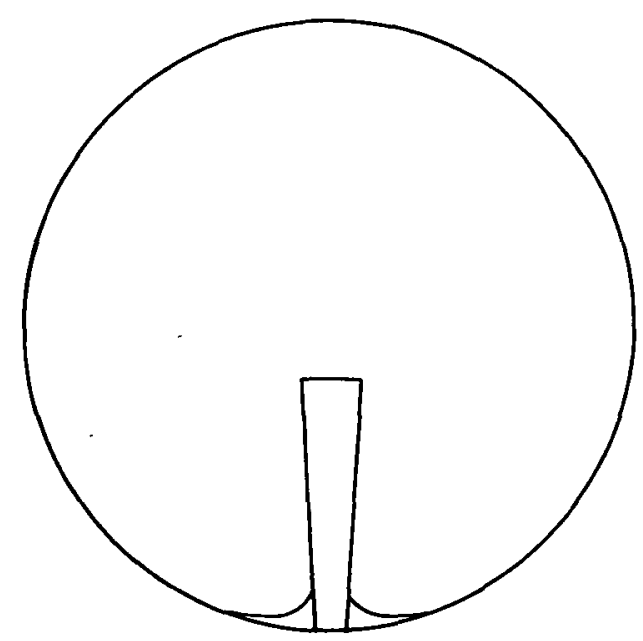

(e) Configuration 4

Eig. III-7 Post Configurations

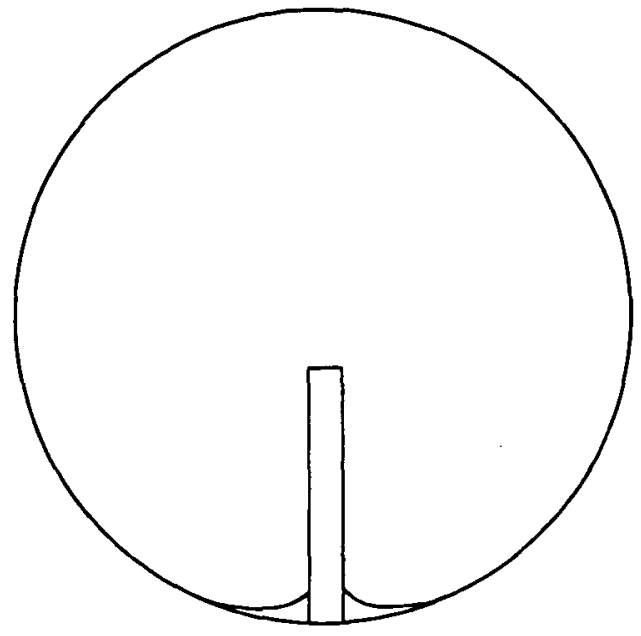

(b) Configuration 1

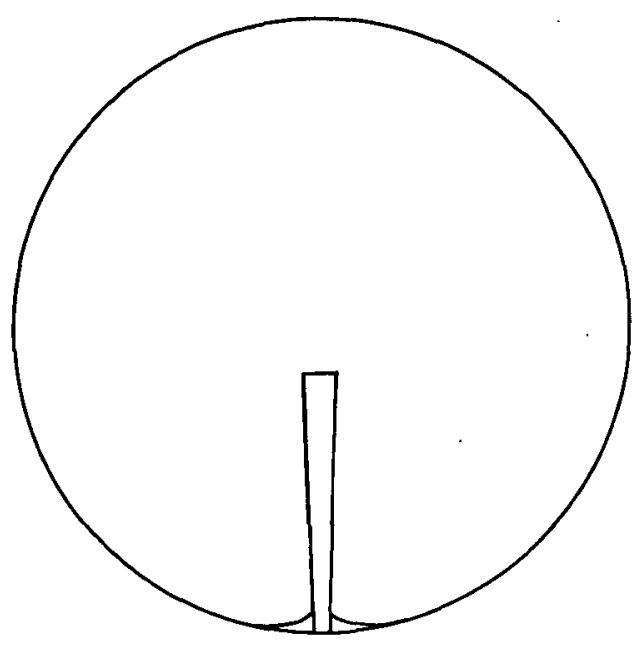

(d) Configuration 3

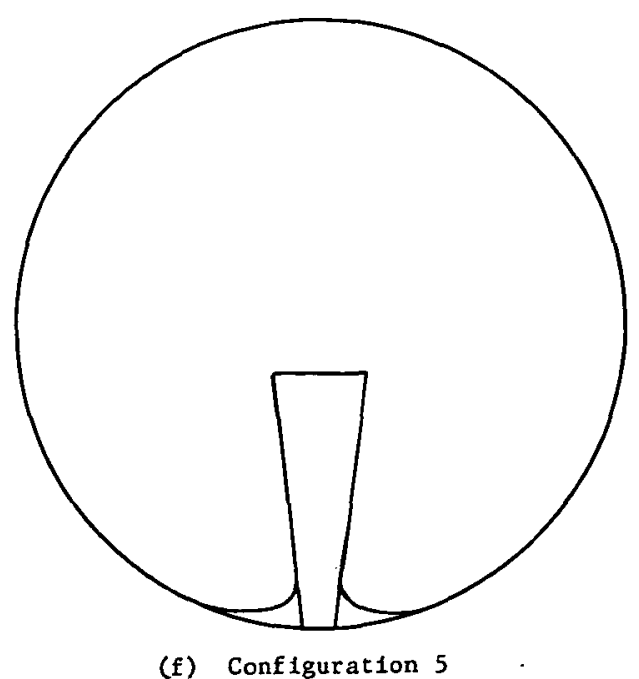

(f) Configuration 5 
The height of the post is determined by the initial ullage volume. An acquisition device should be capable of uniquely positioning the ullage bubble so that mission requirements such as venting, pressurization, and $\mathrm{cg}$ control can be met. If only the near zero $g$ conditions are considered, the curve in Fig. III-8 establishes the height of the post versus ullage volume. The sketch in the figure shows how $h$ is defined; the volume plotted on the horizontal axis is the volume of the spherical bubble as a percentage of the tank volume. In zero $g$, the equilibrium configuration for the ullage is a spherical bubble. A post of height $h$ provides one location between the top of the post and the top of the tank at which the bubble can be positioned. For the simple post, this is a unique location only if the volume of the gas bubble is greater than $12.5 \%\left(\frac{h}{R}=1.0\right)$. When the volume is less than $12.5 \%$ and the diameter of the post is small, there are other locations around the side of the post where the bubble can be spherical. For a. $20 \%$ ullage volume, a post height of $0.83 \mathrm{R}$ would uniquely position the ullage. The height of the post would be $1.26 \mathrm{R}$ for a $5 \%$ ullage, but this post will not guarantee the positioning of the bubble and will only interfere with the positioning of a larger ullage bubble. For this reason, the height of the post for the fuel tank was limited to 1.OR. When the ullage volume is less than $12.5 \%$ in the fuel tank, the location of the bubble is indeterminate.

b. Interface Shapes - Zero-g interface shapes were calculated for the post device in the oxidizer tank. The oxidizer volumes used are those for the Flox tank at various points in the mission. These interface configurations are shown in Fig. III-9.

c. Stability - The axial stability of a liquid interface about a post was analyzed. A critical Bond number, defining the boundary between the stable and unstable regimes, was found by first calculating the free surface shape for a given liquid, geometry, and acceleration, and then mathematically perturbing the surface. A stable or unstable reaction to the pertubation can be detected. By testing various Bond number interfaces, the critical Bond number can be established. This technique was verified by reproducing the critical Bond numbers for a cylinder with contact angles between 0 and $90 \mathrm{deg}$ and annular tanks with various angles and radii. 


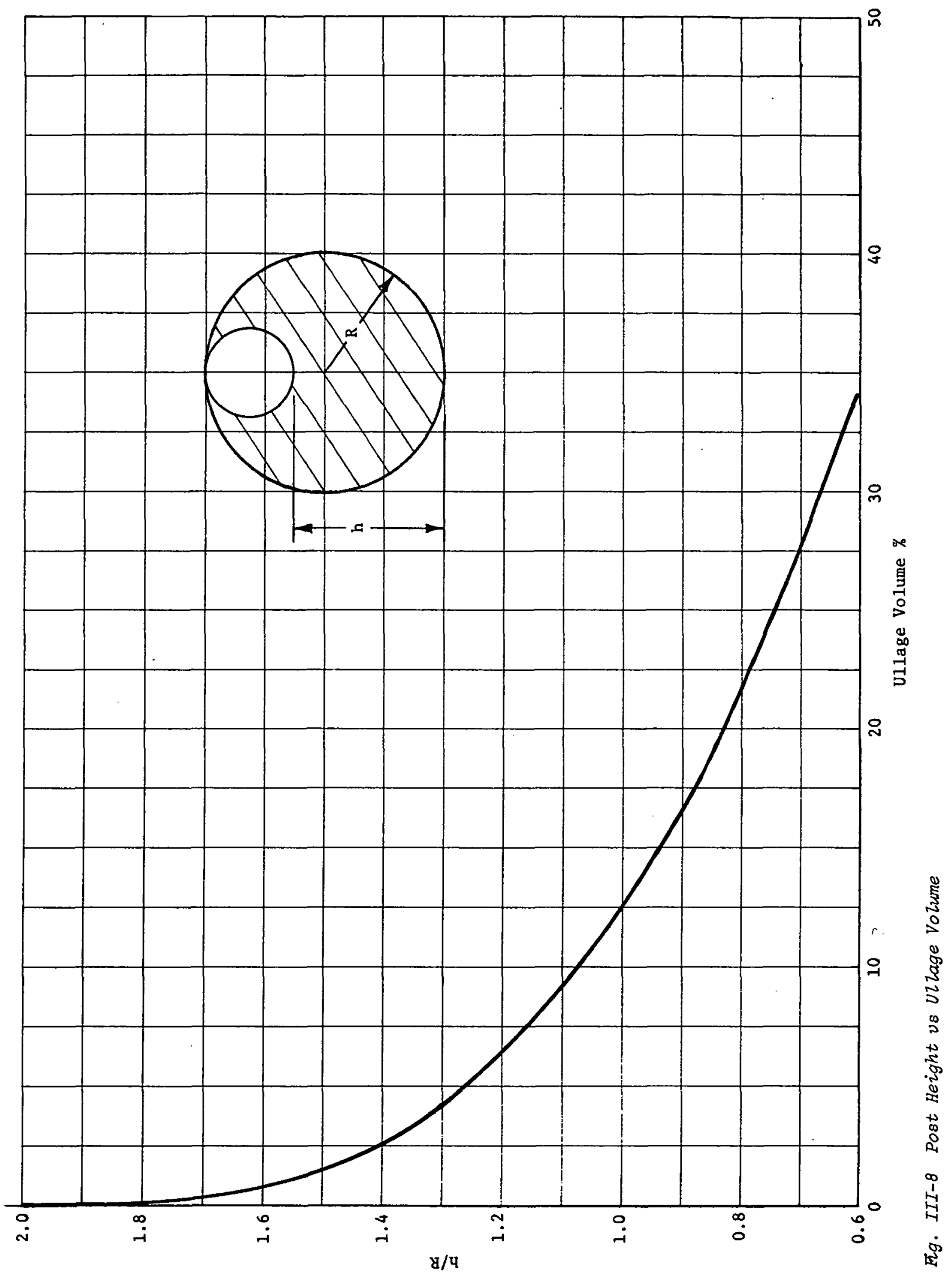

III -13 


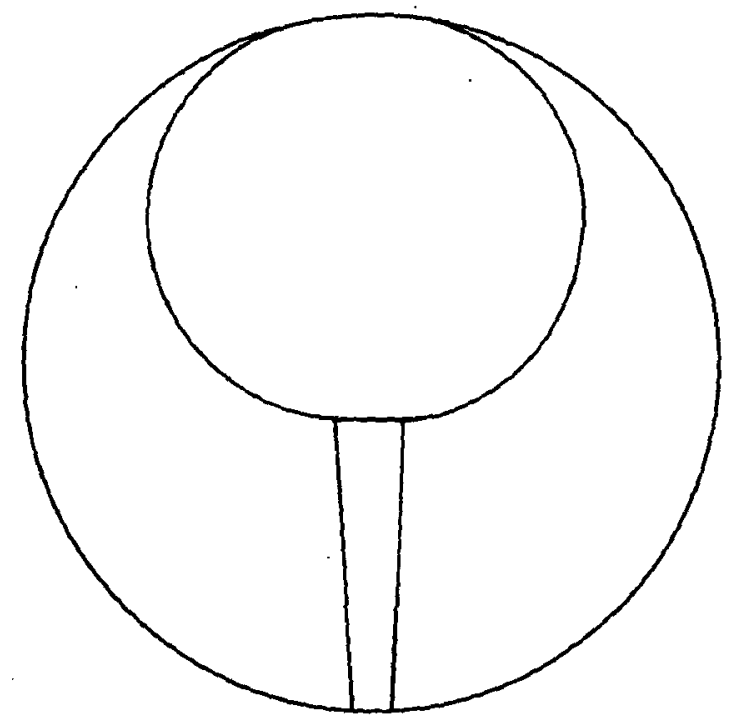

(a) Inttial vilage Volume

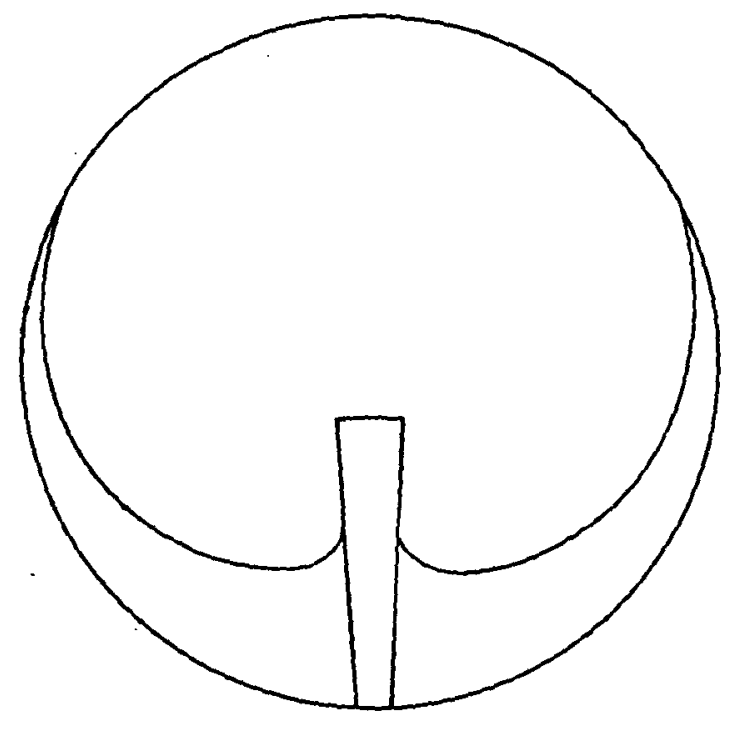

(c) Before Fifth Burn

Fig. III-g Interface Configurations

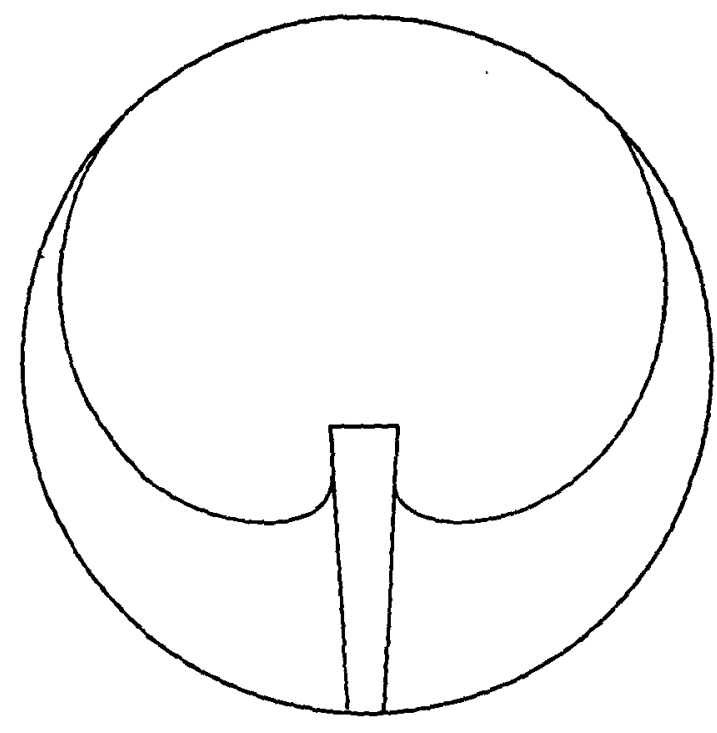

(b) Before Fourth Burn

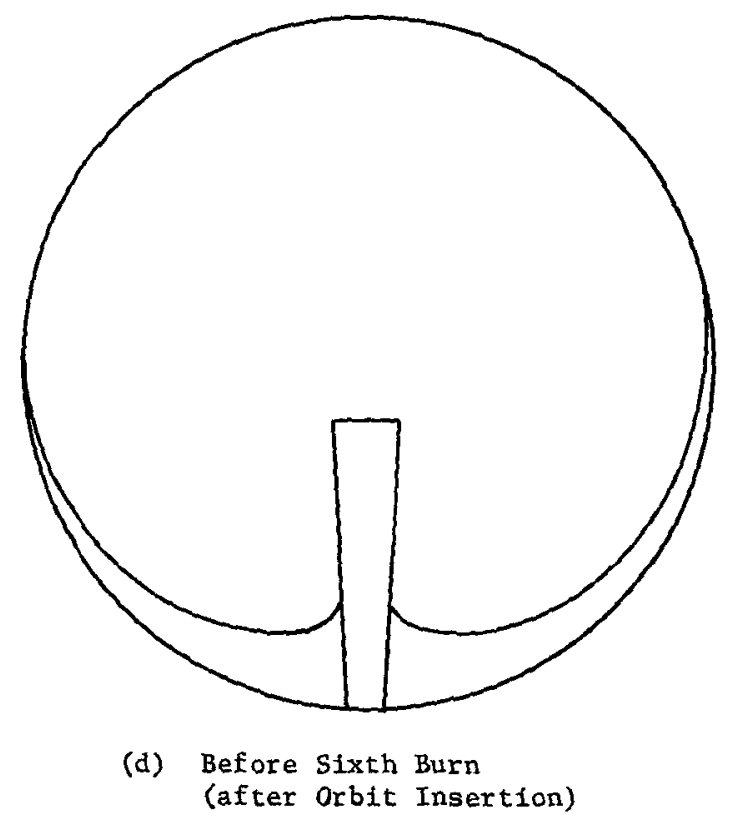


The analysis was then extended to spherical tanks with a cylindrical post located over the outlet. This configuration represents either a post or standpipe propellant acquisition device. Liquid volume, contact angle, and the geometry of the tank were chosen as the variables to define the problem. The Bond number was calculated using the radius of the tank as the characteristic dimension. The acceleration of interest is that acting to move the liquid away from the post to the opposite end of the tank.

Some representative results of the stability analysis are shown in Fig. III-10. Critical Bond number is plotted versus the liquid volume percent for three post diameters and a contact angle of $5 \mathrm{deg}$. A 5-deg contact angle is necessary because of problems inherent in the numerical methods at smaller contact angles. Five degrees does not appreciably change the results from what would be obtained at zero degrees and is a typical compromise used in low-g fluid mechanics calculations.

At either near zero Bond number or very small liquid volume, the liquid will be stable. The greatest variation in stability occurs over the range of volumes and Bond numbers shown. Any combination of Bond number and volume falling below a line for a given post diameter is stable, while any combination above the line is unstable. It can be seen that the effect of increasing the diameter of the post is to increase the stability of the system. If the acceleration acting on a given system is such that the system is unstable, an adjustment in liquid volume will occur. Liquid will flow to the opposite end of the tank until the volume of liquid at the post is reduced to the point at which the interface is stable. This analysis has yet to be extended to tapered posts, but their stability can be estimated from the results for a cylindrical post. The taper will improve the stability of the device, so it would be expected that the post design in Figure III-5 would have a stability curve between those for a $0.1 \mathrm{R}$ diameter post and a $0.2 \mathrm{R}$ diameter post. The results of the experimental program stability tests were consistent with the above analysis. The test results are discussed in Chapter IV.

of the four propellants, Flox will be the least stable because it has the smallest kinematic surface tension (ratio of surface tension to density). The low surface tension of Flox magnifies the problem of designing a device which will provide sufficient interface stability. The maximum axial adverse acceleration acting on the tank is $3 \times 10^{-7} \mathrm{~g}$, which yields a Bond number of 0.064 for Flox. Under this acceleration, approximately a $5 \%$ liquid volume would still be retained about the post. While this amount seems adequate, a complete analysis of the dynamic effects of an engine start with that amount of liquid must be considered. These effects are discussed later in Subsection 8 . 


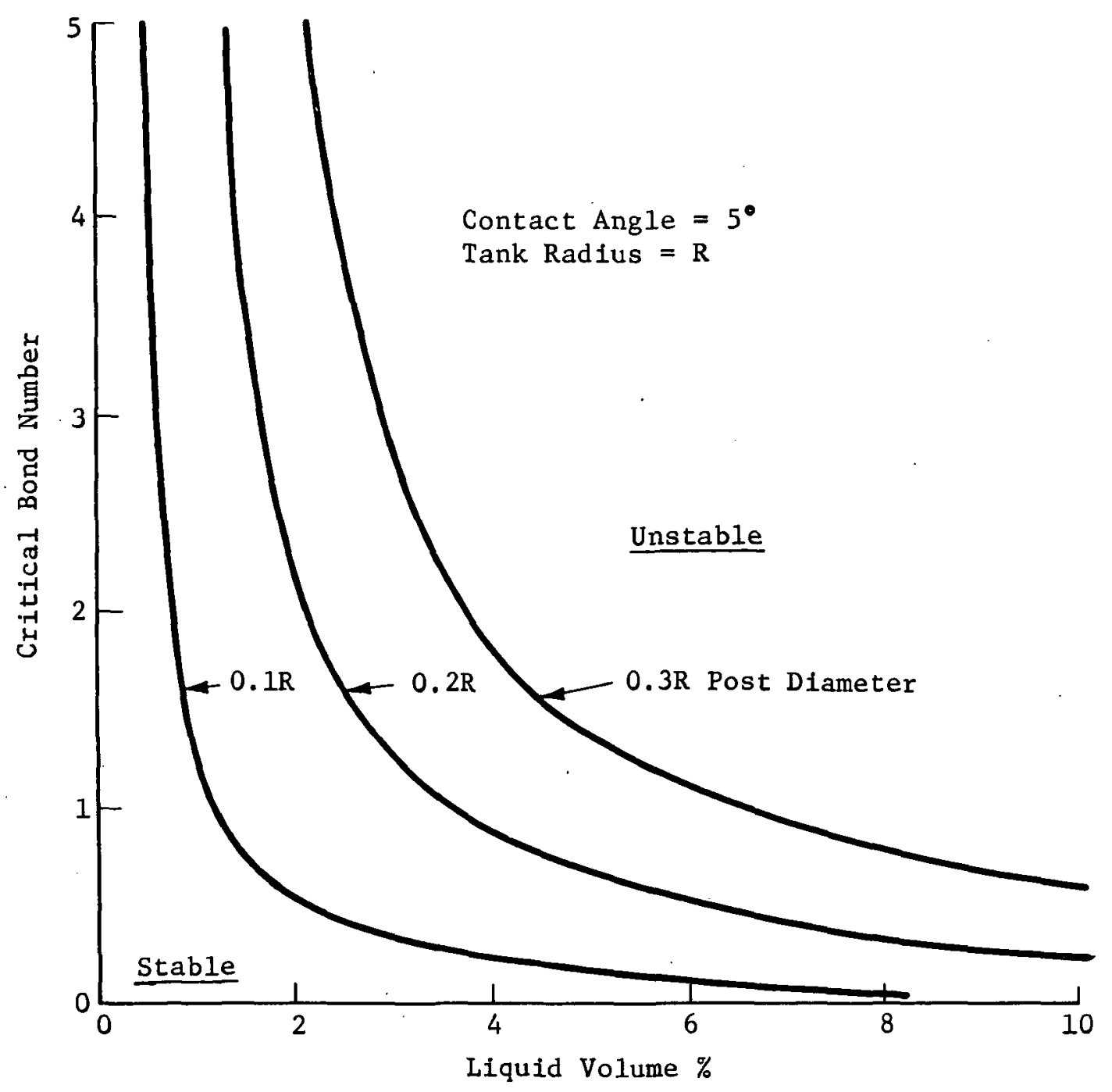

Fig. III-10 Stability of Liquid About a Cylindrical Fruhof Post in a Spherical Tank 
The 1iquid interface about the post will become "stuck" when the volume of liquid reaches a certain percentage of the tank volume. An example of a stuck interface is shown in Fig. III-9(a) while the contact angle is satisfied at both the post and the tank wall in Fig. III-9(b). In Fig. III-9(a), the interface is stuck at the top of the post and the angle formed by the interface and the post is much larger than the contact angle.

The stability of a stuck interface, relative to an interface that satisfies contact angle, is significant in the design of the acquisition devices. Part of the stability analysis was repeated for the stuck interface. The $0.2 \mathrm{R}$ diameter post was used for comparison, but the height of the post was 1 imited to $0.25 \mathrm{R}$. For a volume of $1.85 \%$ at the critical Bond number, the interface has just become stuck. It forms an angle of $5^{\circ}$, the contact angle, with the post, but a slight increase in volume will increase that angle. Therefore, the same critical Bond number is obtained at this point as was obtained previously. As shown in Figure III-11, increasing the volume of liquid with a stuck interface produces a significant decrease in the critical Bond number in comparison with the contact angle satisfied interface. At liquid volumes near $6 \%$ of the tank volume, a stuck interface is about $30 \%$ less stable than an interface that satisfies contact angle.

Two points on the stuck interface curve are illustrated in Fig. III-12. Figure III-12(a) shows the contact angle satisfied condition just prior to the point where the interface first becomes stuck as the volume is increased. The configuration shown in Fig. III-12(b) represents a stuck interface condition with $7.6 \%$ liquid volume which forms an angle of $50^{\circ}$ with the post.

This analysis demonstrates that a stuck interface is less stable than an interface that satisfies contact angle, all other factors remaining constant. This should be kept in mind when designing any acquisition device. If interface stability is critical for a given range of liquid volumes, the dimensions of a device could be increased. This would increase the liquid volume at which the interface becomes stuck and improve the stability of the device over the liquid volume range of interest. 


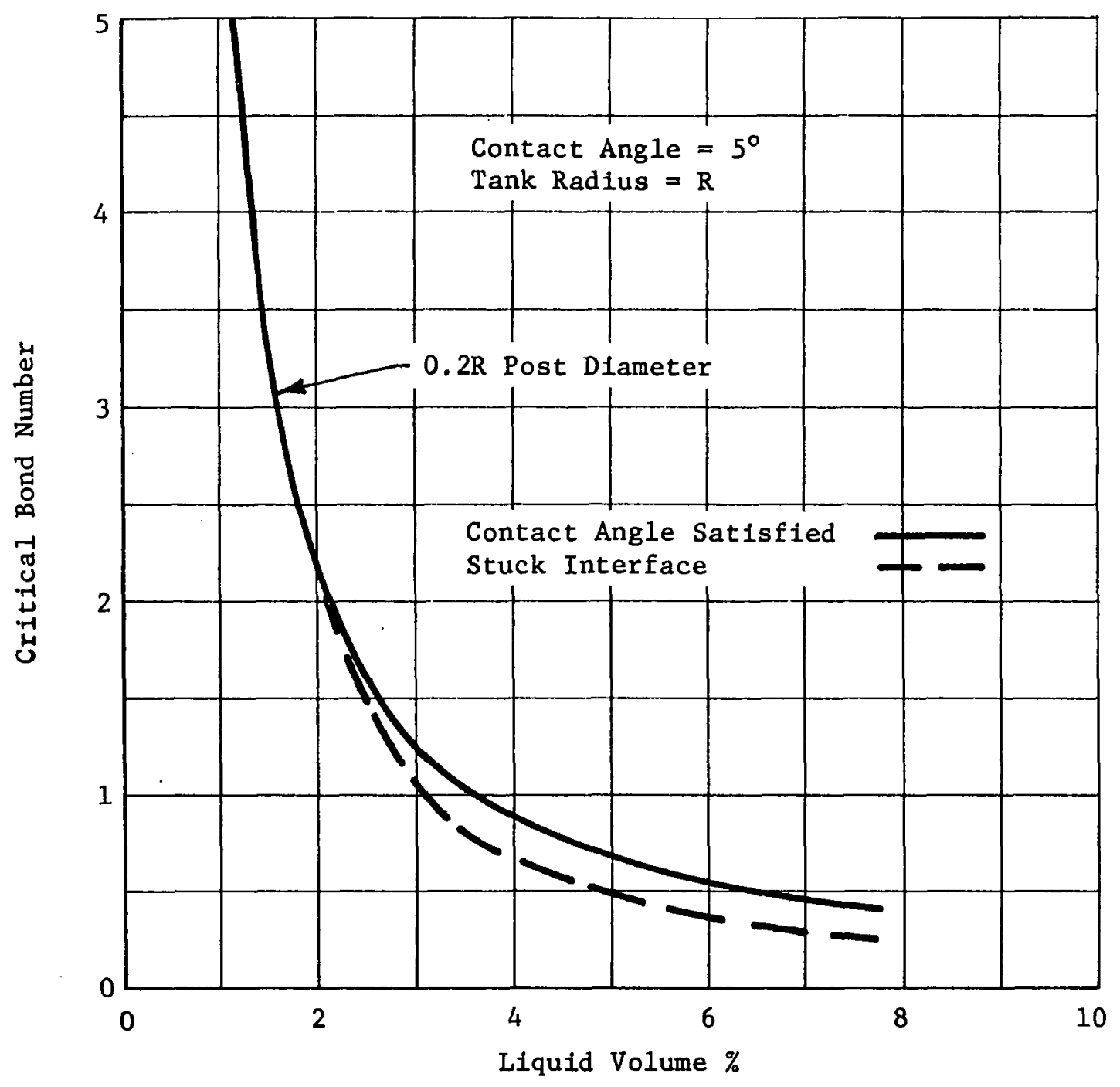

Fig. III-11 Effect of a Stuck Interface on Stability 


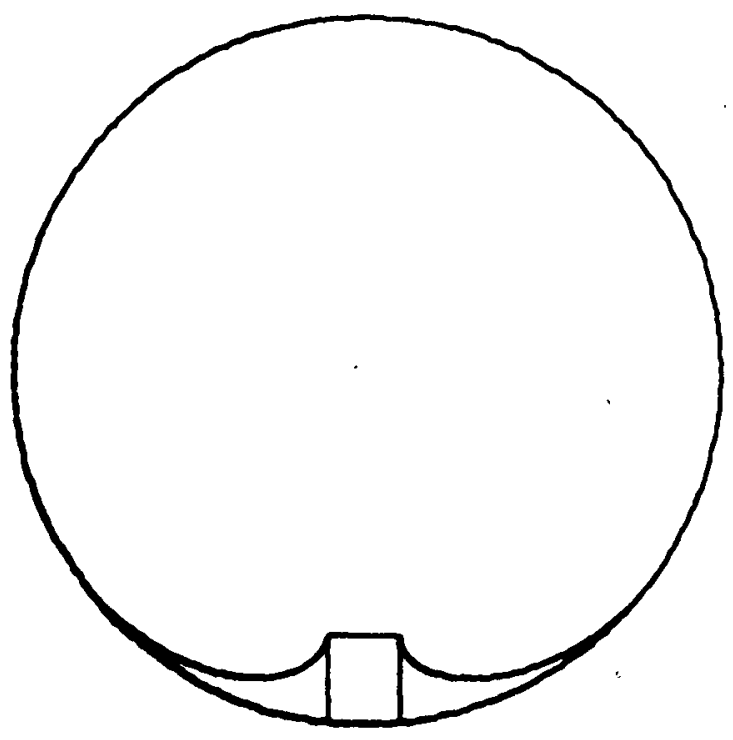

(a) Contact Angle Satisfied

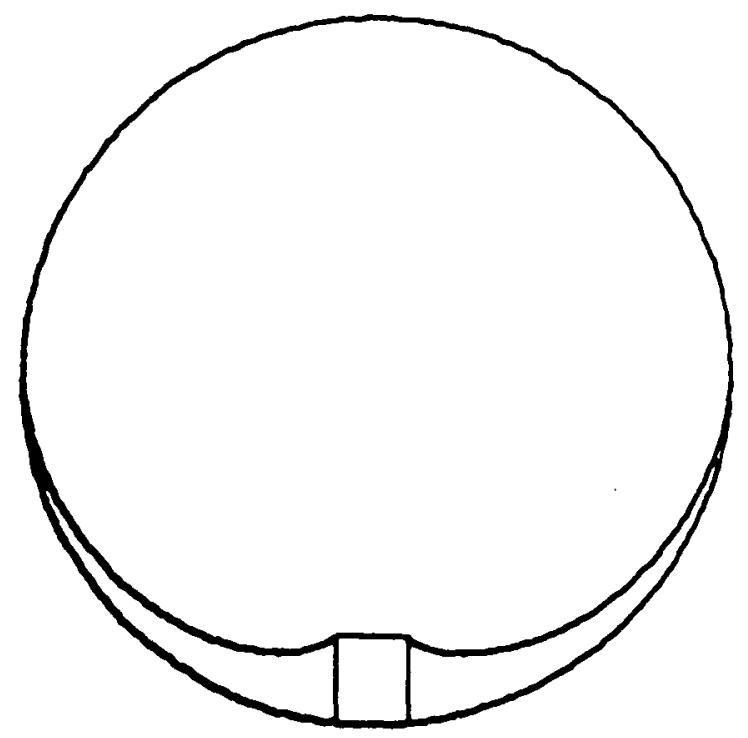

(b) Stuck Interface

Fig. III-12 Low-g Interfaces about Post 
Lateral stability of the post concerns the ease with which the centered ullage bubble can be laterally displaced from that location. As the bubble is displaced, it must be distorted from its spherical shape, and capillary forces will be produced that tend to recenter the bubble. Lateral stability of the post can not be analyzed as easily as axial stability. Some quantitative data were provided by the stability tests conducted in the drop tower (see Chapter IV). These tests also demonstrated the relative lateral stability of the post with respect to other devices. When compared with the other candidates, the post has little effect on the shape of the bubble as it is displaced from the centered position. Therefore, it has the least lateral stability, and any lateral disturbance would produce an offset ullage. However, the post would be capable of recentering the bubble after the disturbance was removed.

Based on the analysis of the post operation, it was concluded that this device is not as capable as the other candidate concepts in meeting the mission requirements. The most significant weaknesses of the post device are:

1) The device for the fuel tank is not capable of positioning the ullage bubble at volumes less than $12.5 \%$;

2) The stability of the device is lower than any of the other candidate devices;

3) At contact angles greater than $2^{\circ}$, communication channels will not function. This problem is discussed in detail in the analysis of the channels presented later in Subsection 7.

Because of these problem areas, the post device was eliminated as a candidate prior to the actual evaluation phase.

\section{Standpipe}

The standpipe is another Category $\mathrm{C}$ device. It is essentially a large-diameter, hollow post. Standpipe designs for the oxidizer and fuel tanks are shown conceptually in Fig. III-13 and III-14, respectively. Openings are provided about the base of the standpipe so it can fill with liquid.

a. Design - The post analysis showed that increasing the diameter caused the liquid to be held closer about the device. For the post, large diameters are a disadvantage because the volumetric efficiency of the system is significantly decreased. With the standpipe, this effect is slight, permitting the use of larger diameters. 


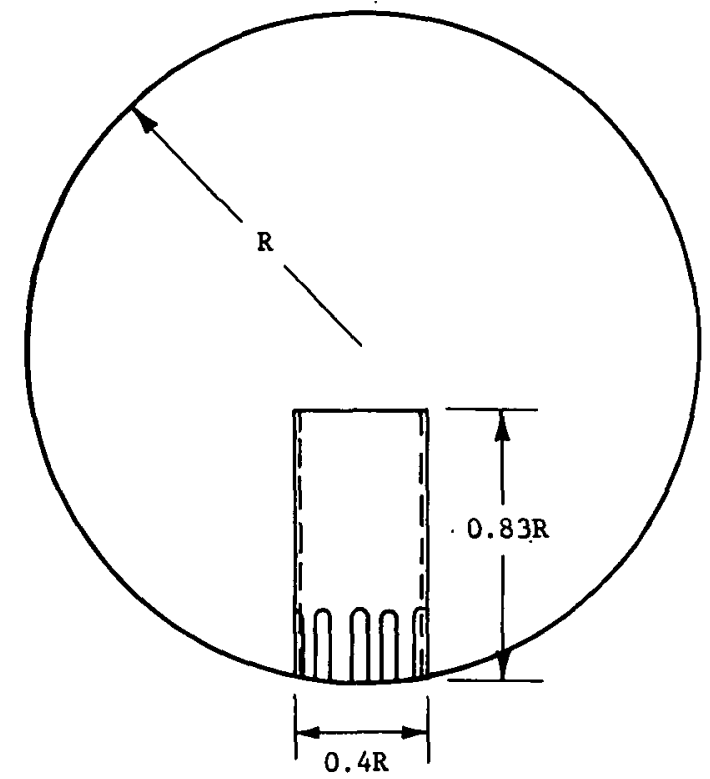

Figure III-13 Standpipe for Oxidizer Tank

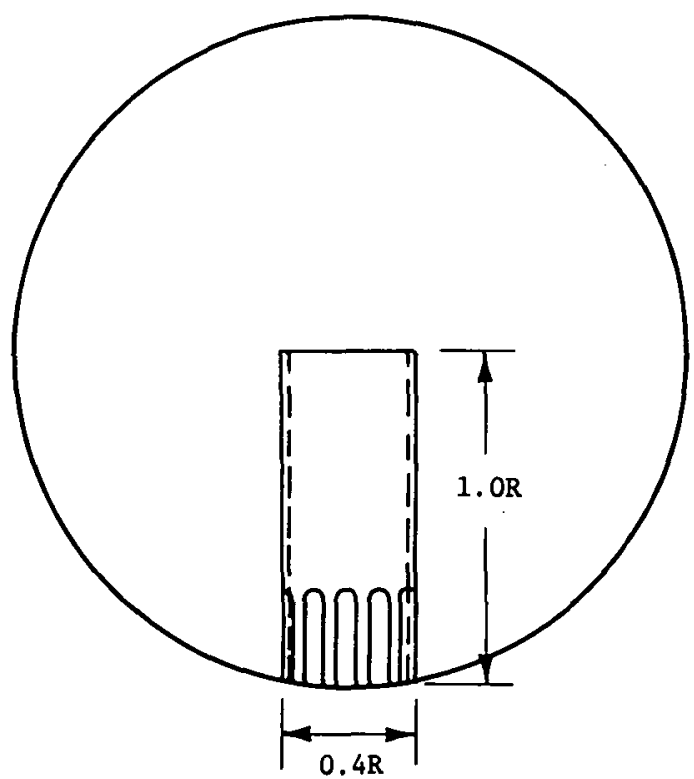

Figure III-14 Standpipe for Fuel Tank 
Design of the outlet for the standpipe presents one of the disadvantages of the large-diameter device. At small liquid volumes, liquid collects on the inside and outside of the standpipe at the point it meets the tank wall. This toriodal volume must be fed to an outlet on the tank centerline. Some form of manifold 1ocated at the base of the standpipe would be required.

Height of the standpipe is determined in a manner similar to that used for the post. The curve of height versus ullage volume in Fig. III-8 also applies to the standpipe. For the $20 \%$ initial ullage volume of the oxidizer tank, a height of $0.83 \mathrm{R}$ will uniquely position the bubble. Positioning of the bubble for a $5 \%$ ullage presents a problem for the standpipe, as it does for the post. However, the diameter of the standpipe has some effect on the positioning. A $5 \%$ spherical bubble has a diameter of $0.74 \mathrm{R}$. This size bubble is always tangent to the tank wall and a concentric sphere of $0.26 \mathrm{R}$ radius, as shown in Fig. III-15. Therefore this bubble would always be in its equilibrium, spherical configuration wherever it is positioned between the wall and the $0.26 \mathrm{R}$ radius sphere. If the standpipe has a radius larger than $0.26 \mathrm{R}$, a unique position in which the bubble can be spherical is formed. For example, a $0.3 \mathrm{R}$ radius standpipe, drawn with dashed lines in Fig. III-15, allows the bubble to be in its equilibrium, spherical configuration at only the centered position. But further analysis of the $0.3 R$ radius standpipe shows that there are other stable states, although not as stable as the spherical bubble.

If the bubble is initially located off to the side of the standpipe, it will be distorted since a spherical bubble can not fit into the space available. As the capillary forces push the bubble toward the top of the tank, the area of the path becomes constricted. The area between the standpipe and the tank wall reaches a minimum, labeled $A_{\text {min }}$ in Fig. III-15, at the top of the standpipe. The base of the standpipe produces capillary forces that act to center the bubble, but the constriction at the top of the standpipe also produces capillary forces that oppose the centering. Therefore, an equilibrium position at which the forces are balanced will be reached when the bubble is offset to the side of the standpipe. The bubble will center only if the area of the path continues to increase as the bubble is centered. Otherwise other stable states will exist. The drop tower tests demonstrated the existence of these other stable states for the standpipe. Photos of the bubble position during testing with a standpipe are in Chapter IV. 


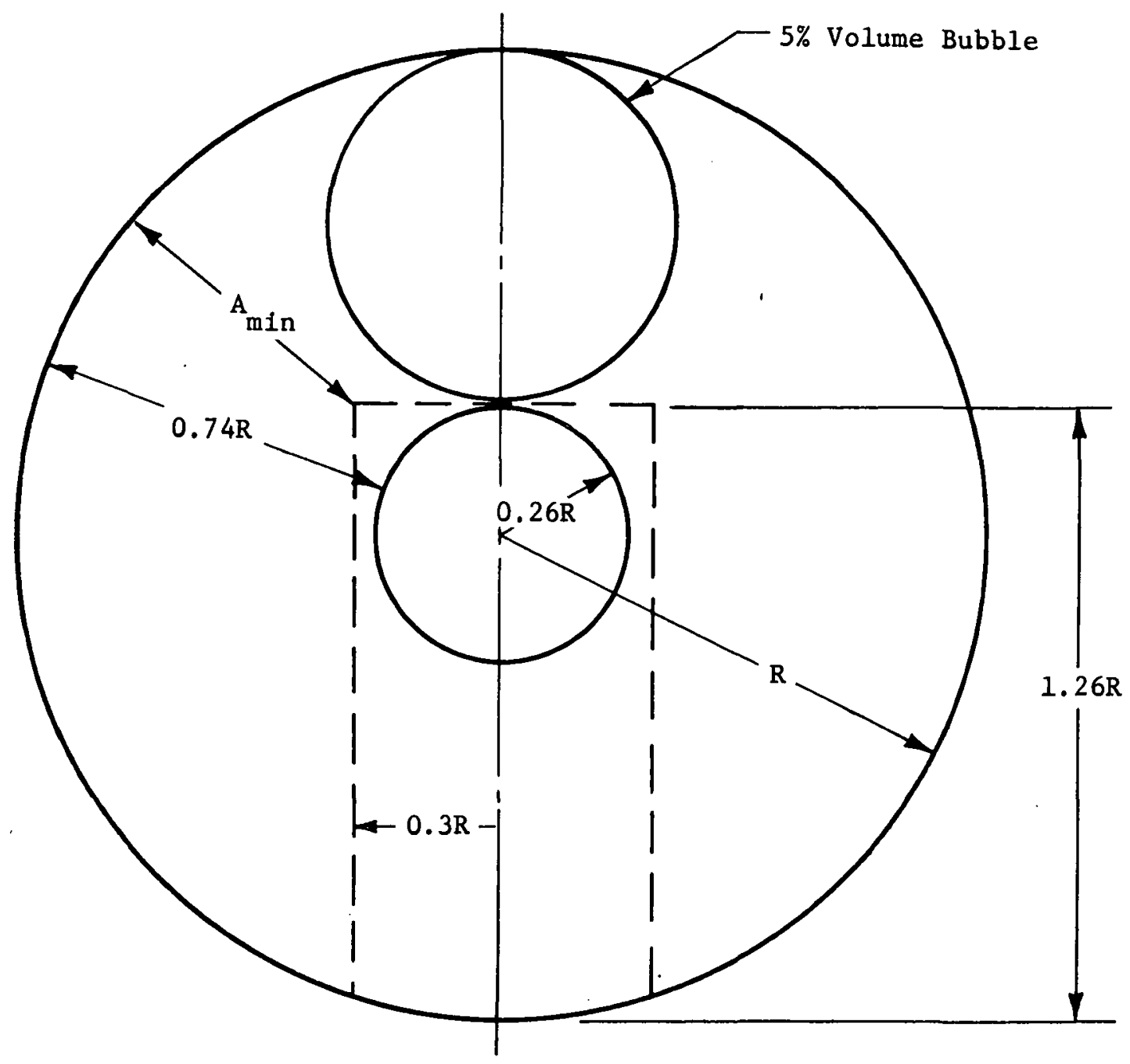

Fig. III-15 Positioning of a 5\% Bubble 
For the above reasons, it can be seen that extending the standpipe beyond a height of $1.0 R$ in the fuel tank has no advantage. Centering of the ullage bubble must be sacrificed for ullage volumes less than $12.5 \%$ with the standpipe system. The internal volume of the standpipe for the oxidizer and fuel tanks is $2.5 \%$ and $3 \%$, respectively.

b. Interface Shapes - The shape of the interface about the standpipe would be similar to the interfaces shown previously for the post, except that the curvature would be increased due to the larger diameter of the standpipe. At the larger liquid volumes, the standpipe will be full of liquid. The curvature of the interface within the standpipe is greater than the curvature of the interface outside the standpipe. This causes the pressure of the liquid to be less within the standpipe, so filling must take place to equalize pressures. Only when the interface of the liquid inside reaches the top of the standpipe and becomes stuck will the curvature decrease and establish pressure equilibrium.

When the liquid volume is on the order of the volume of the standpipe, however, equilibrium between liquid inside and outside the standpipe can be attained without the internal interface becoming stuck. Inside the standpipe, the curvature of the interface remains constant for any volume, as long as the interface is not in contact, with the bottom of the tank. Outside, the curvature varies inversely with the volume. Given the curvature of the interface inside the standpipe, liquid will transfer either into or out of the standpipe until pressure equilibrium is established. This equilibrium condition was calculated for three standpipes of different diameters and the results are listed in Table III-2.

Table III-2 Volume of Liquid Outside Standpipe

\begin{tabular}{|l|l|}
\hline $\begin{array}{l}\text { Standpipe Diameter } \\
\text { Ratio, } \frac{\mathrm{d}}{\mathrm{R}}\end{array}$ & $\begin{array}{l}\text { Liquid Volume Outside } \\
\text { Standpipe, Percent of } \\
\text { Tank Volume }\end{array}$ \\
\hline 0.3 & 0.031 \\
0.4 & 0.091 \\
0.5 & 0.221 \\
\hline
\end{tabular}

For the $0.4 \mathrm{R}$ diameter ratio standpipe, a small percentage of the liquid will be outside the pipe, while most of the liquid will remain inside. The equilibrium condition for this standpipe is shown in Fig. III-16. 


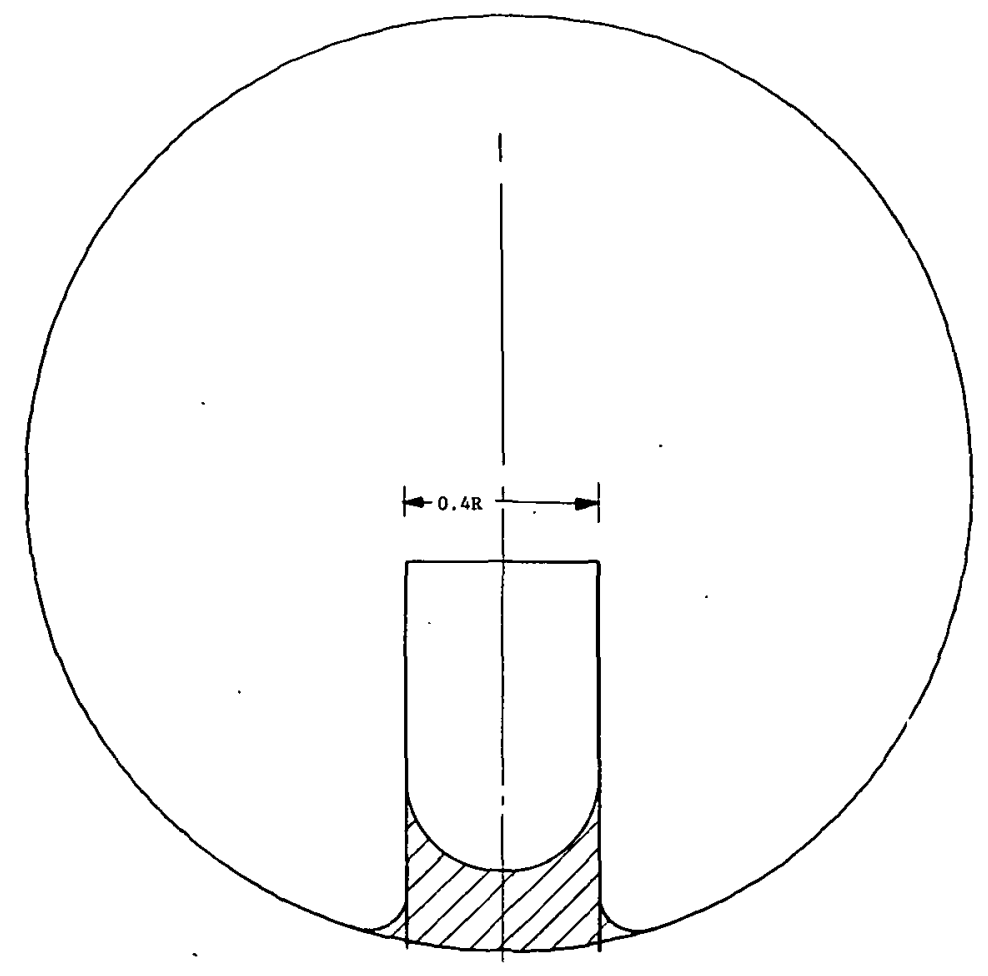

Fig. III-16 Equilibrium Configuration for the Standpipe

c. Stability - Axial stability of the standpipe can be established directly. Within the pipe, the critical Bond number based on the radius of the standpipe is 0.84 for a zero contact angle. Redefining the Bond number by using the tank radius, based on a $0.4 R$ diameter standpipe, the critical Bond number is 21 . A very stable reservoir of liquid is provided by the standpipe.

Outside the standpipe, the stability is given by the same curve used for the post (Fig. III-10). The critical Bond number is 2.5 for a liquid volume of $5 \%$ outside the standpipe.

At low ullage volumes, the lateral stability of the standpipe is much like that of the post. Since the standpipe has a larger diameter, it applies a larger centering force for a given displacement of the ullage bubble. At ullage volumes less than $12.5 \%$, the device in the fuel tank cannot center the bubble, so it has no lateral stability under those conditions. Due to the configuration of the device, liquid located inside the standpipe is very stable under the effect of lateral accelerations. 
5. Post with Fingers

This device is a composite of the post, previously discussed in Subsection 3, and a finger device. By themselves, the fingers were not considered adequate because their ability to position liquid is weak and they provide very little stability. But, when the two devices are combined, one device makes up for the weaknesses of the other. The devices for the oxidizer and fuel tanks are shown in Fig. III-17 and III-18, respectively.

Four fingers, each a metal rod bent to a specified profile, are used with the post for the oxidizer tank. The profile extends from the base of the post to the point at which it would contact the spherical ullage bubble. The ullage bubble is uniquely positioned by the post alone, so the fingers only reinforce that positioning.

Six fingers are used with the post for the device in the fuel tank. With the fingers, the only point at which the bubble can be spherical is the centered position. The height of the post has been extended to $1.26 \mathrm{R}$ in this application. Other stable bubble positions are possible though, due to the weak positioning effect of the fingers. If the bubble is initially centered, the fingers will assist the post in maintaining the bubble in that position against a lateral disturbance. Under ideal conditions in the drop tower tests, the post with fingers performed rather well (see Chapter IV).

Stability of the device in the axial direction is the same as the post by itself. In the lateral direction, there would be some improvement due to the fingers. 


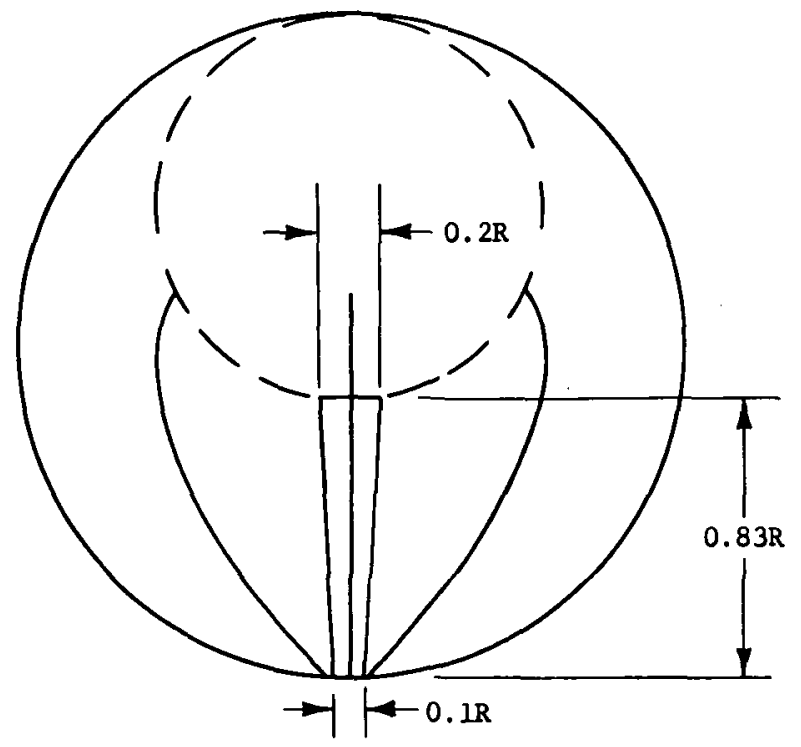

Fig. III-17 Post with Fingers for

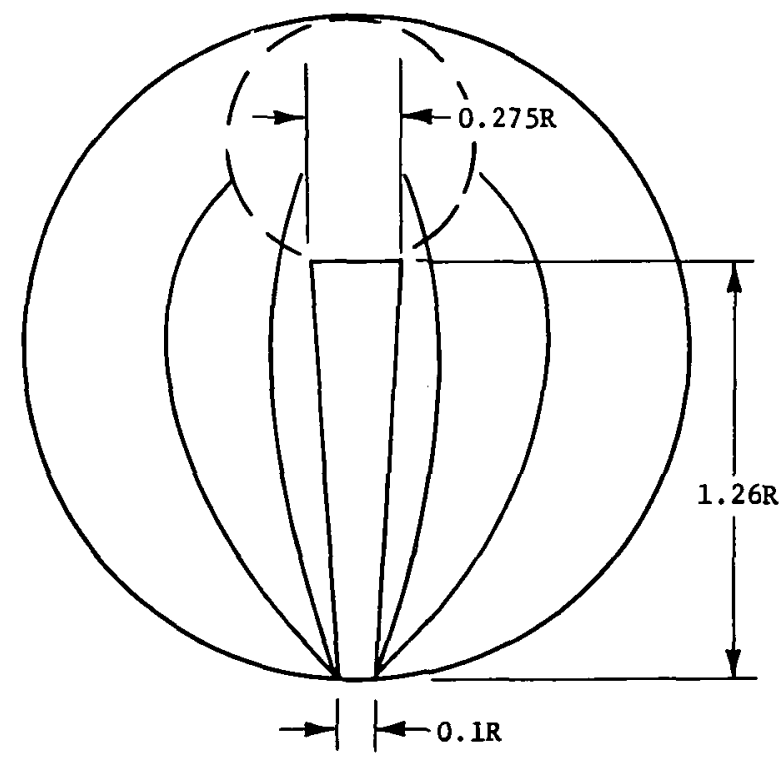

$\begin{array}{cl}\text { Fig. III-18 Post with Fingers for } & \text { Fuel Tank } \\ & \text { Ful }\end{array}$ 
6.

Vanes

This Category $C$ device consists of a number of vanes radiating outward from a small central post, as shown in Fig. III-19 and III-20. Only the profile of two of the six vanes is shown for clarity. The post serves as a structural member and has little effect on the operation of the device.

a. Design - The primary variables involved in the design of vane devices are the number of vanes and the vane profile. Height of the device is established by Fig. III-8, as it was for the other Category $\mathrm{C}$ devices.

1) Vane Profile Limits - The objective in designing the vane profile is to make the centered position the only position in which the bubble will be in equilibrium. A bubble located elsewhere will be acted upon by capillary forces that will pump it toward the centered position. There are two limits applicable to the vane profile; these are an inner profile 1 imit that must be exceeded and an outer profile limit whose application is optional.

The inner limit establishes a profile that allows the ullage bubble to be tangent to the tank wall and just touch the vanes. The centered position becomes a unique ullage equilibrium position by always keeping the vane profile outside the inner limit, except for the centered position. This inner profile limit is only applicable to ullage volumes less than $12.5 \%$. Even a simple post will uniquely position the bubble when the volume is greater than $12.5 \%$.

The relationship between the ullage bubble and the vanes that establish the inner limit is shown in Fig. III-21(a) and (b). In Fig. III-2l(b), the angle $\alpha$ is a function of the number of vanes and the angle $\phi . \mathrm{L}_{\min }$ is the length of the vane, measured from the center of the tank, such that two vanes will contact the given ullage bubble. The following equations define the inner limit:

$$
\begin{aligned}
& \mathrm{L}_{\min }=(R-r) \cos \frac{\alpha}{2}-\sqrt{\mathrm{r}^{2}-\sin ^{2} \frac{\alpha}{2}(R-r)^{2}} \\
& \alpha=\theta \cos \phi \\
& r=\left(\frac{3 V}{4 \pi}\right)^{1 / 3}
\end{aligned}
$$

where

$\theta=$ angle between vanes when $\phi=0$,

$\mathrm{V}=\mathrm{ullage}$ volume as a percent of tank volume. 


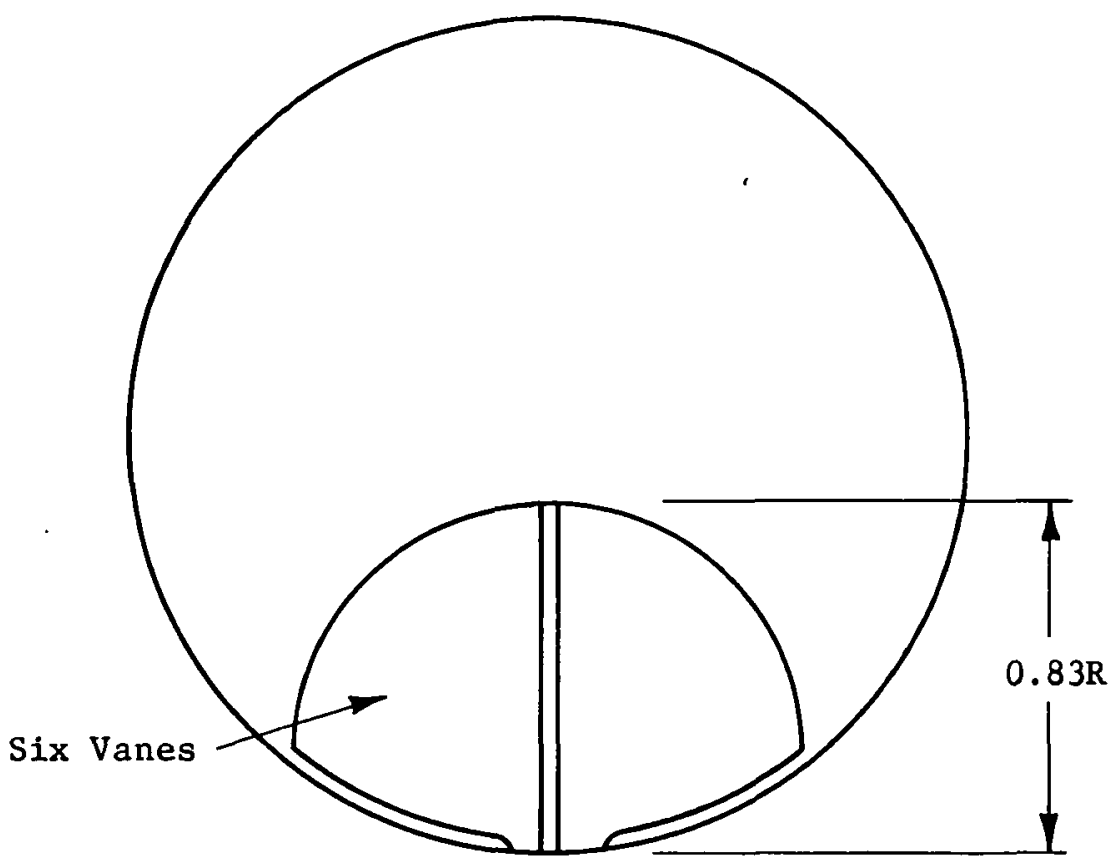

Fig. III-19 Vanes for Oxidizer Tank

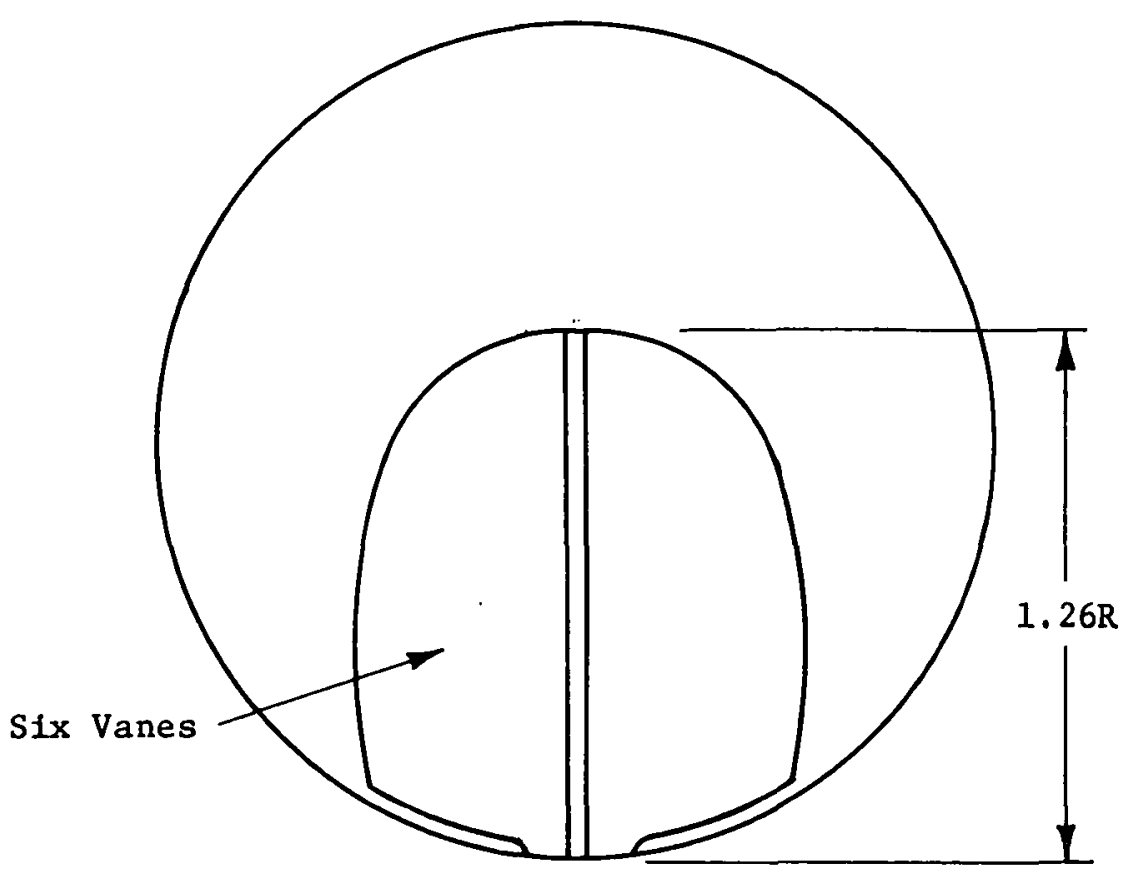

Fig. III-20 Vanes for Fuel Tank 


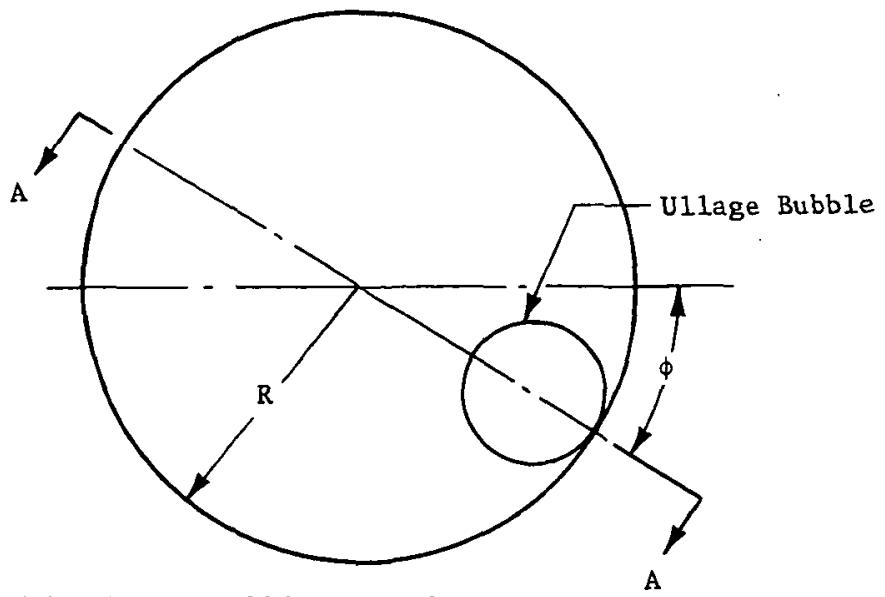

(a) U1lage Bubble in Tank

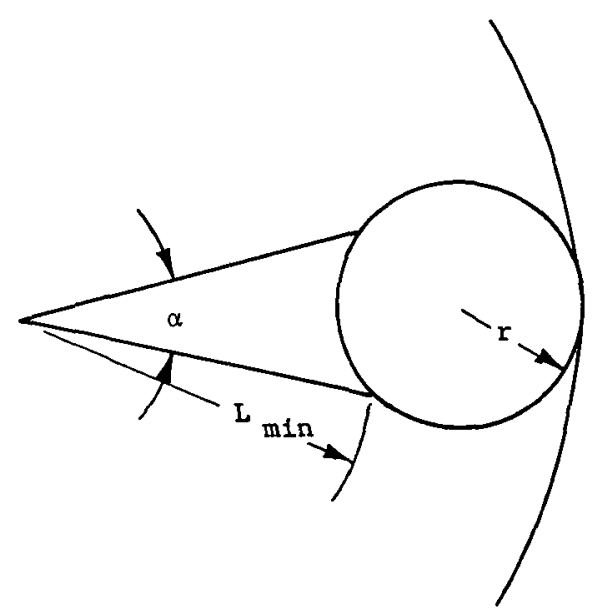

Section A-A

(b) Inner LImft Geometry

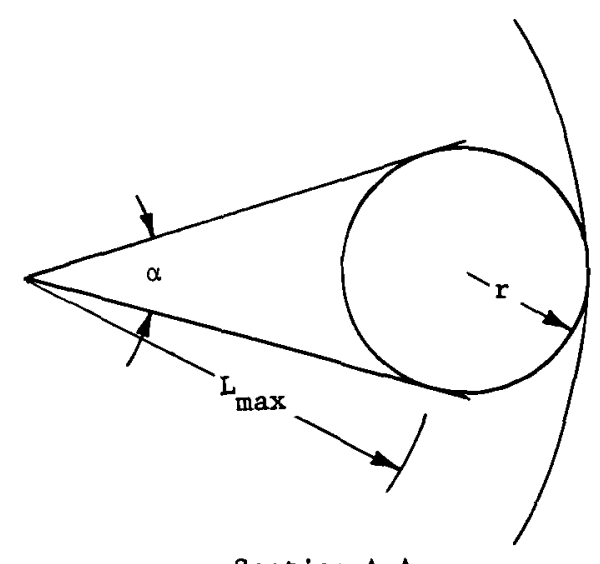

Section A-A

(c) Outer Limit Geometry

Figure III-21 Vane Profile Limits

III-30 
The other variables are defined in Fig. III-21. The length $\mathrm{L}_{\text {min }}$ is plotted versus $\phi$ in Fig. III-22 for an ullage volume of $5 \%$ and various numbers of vanes. The limit is symmetrical about the centerline of the tank.

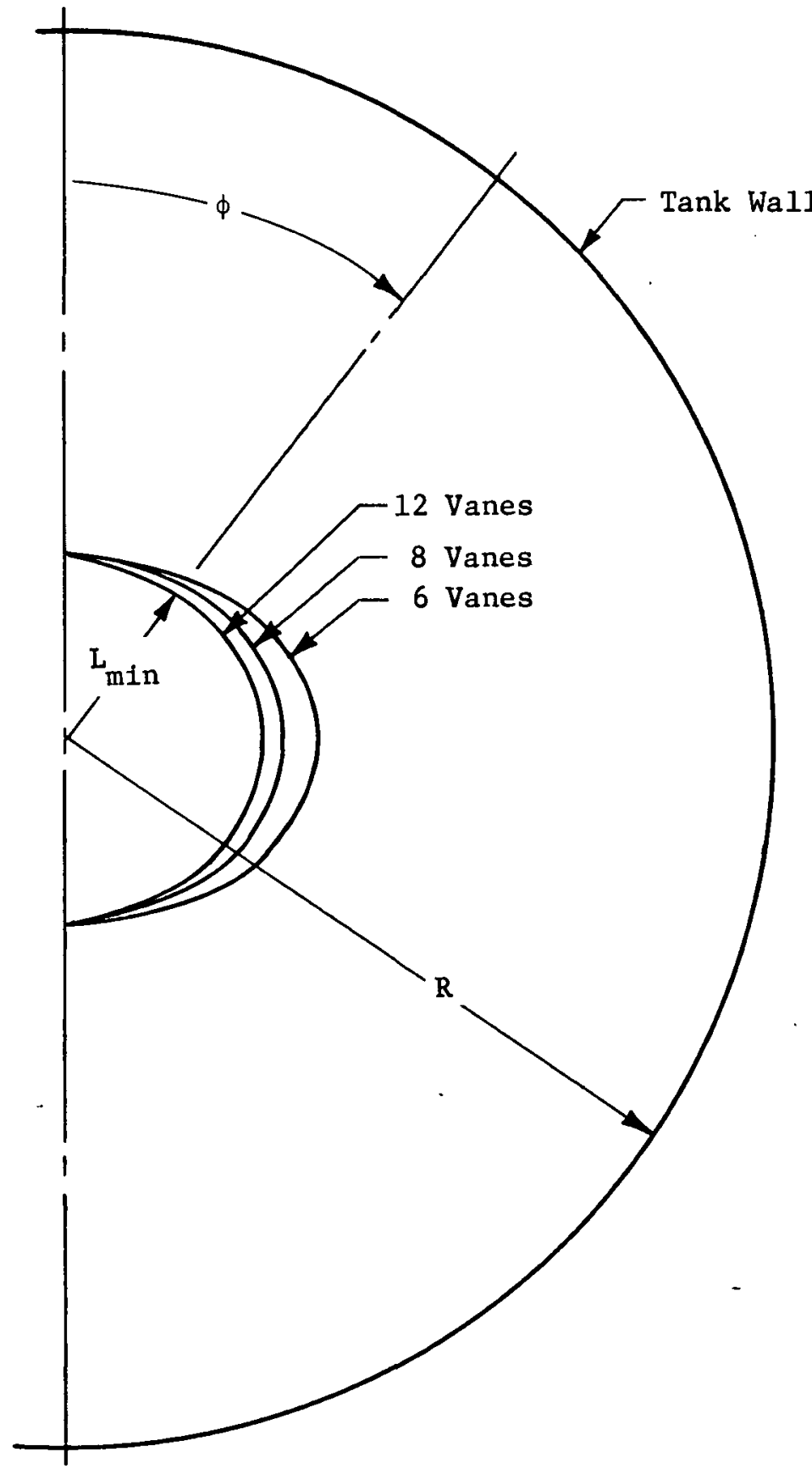

Fig. III-22 Inner Profize Limit for 5\% Ulzage 
A phenomena called "bubble breakup" defines an outer limit to the vane profile. Application of this limit to the design of the vane depends on a number of factors that will be discussed after the limit has been defined. Breakup of the ullage bubble was demonstrated with vaned devices in the drop tower tests. To cause the breakup to occur during the drop test, the vane devices were inverted and small ullage volumes ( 5 to $20 \%$ ullage) were used. At the beginning of the test, the ullage is located at the base of the device. Once the system enters low $g$, liquid fills the gap between the edge of the vane and tank wall, splitting the ullage into a number of bubbles before the whole bubble could be pumped away from the outlet. This ullage splitting can occur so that there will be a bubble between each vane. These small bubbles can be spherical and still fit within the vanes, so that no capillary forces act to move them further from the outlet. These bubbles would be buoyed away from the tank outlet and would not be ingested when the engine starts, as discussed later in Subsection 8. However, should a breakup of the ullage occur, there would no longer be a centered ullage for the purposes of venting and pressurization. An outer vane profile limit, within which this breakup will not occur, can be established. Figure III-21(a) and (c) illustrates the geometry of the criteria. In this case, the point at which the circle is tangent to the vane determines the length $L_{\max }{ }^{-}$When the length of the vane at angle $\phi$ is less than $L_{\text {max }}$, pinchoff of the ullage will not occur, and the bubble will be pushed away from the outlet without breaking up.

The criteria for the maximum length can be expressed as follows:

$L_{\max }=\frac{\mathrm{R} \cos \left(\frac{\theta}{2} \cos \phi\right)}{\sin \left(\frac{\theta}{2} \cos \phi\right)+1}$

where the variables are the same as those used to define the inner limit. The outer limit is only a function of the number of vanes, as shown in Fig. III-23. Vanes were designed with profiles that were within the outer limit. These were tested in the drop tower and no breakup of the ullage bubble occurred.

The profile of the vane in the vicinity of the outlet can go beyond the outer limit without causing breakup. An example is the profile for an eight-vane system shown in Fig. III-24. When the profile exceeds the limit, as shown, and extends so that it comes into contact with the tank wall, breakup of the ullage does not occur. The small protion of the vane device, which is outside the limit, rapidly fills as the system enters a low-g environment, so it does not affect the orientation of the bubble. 


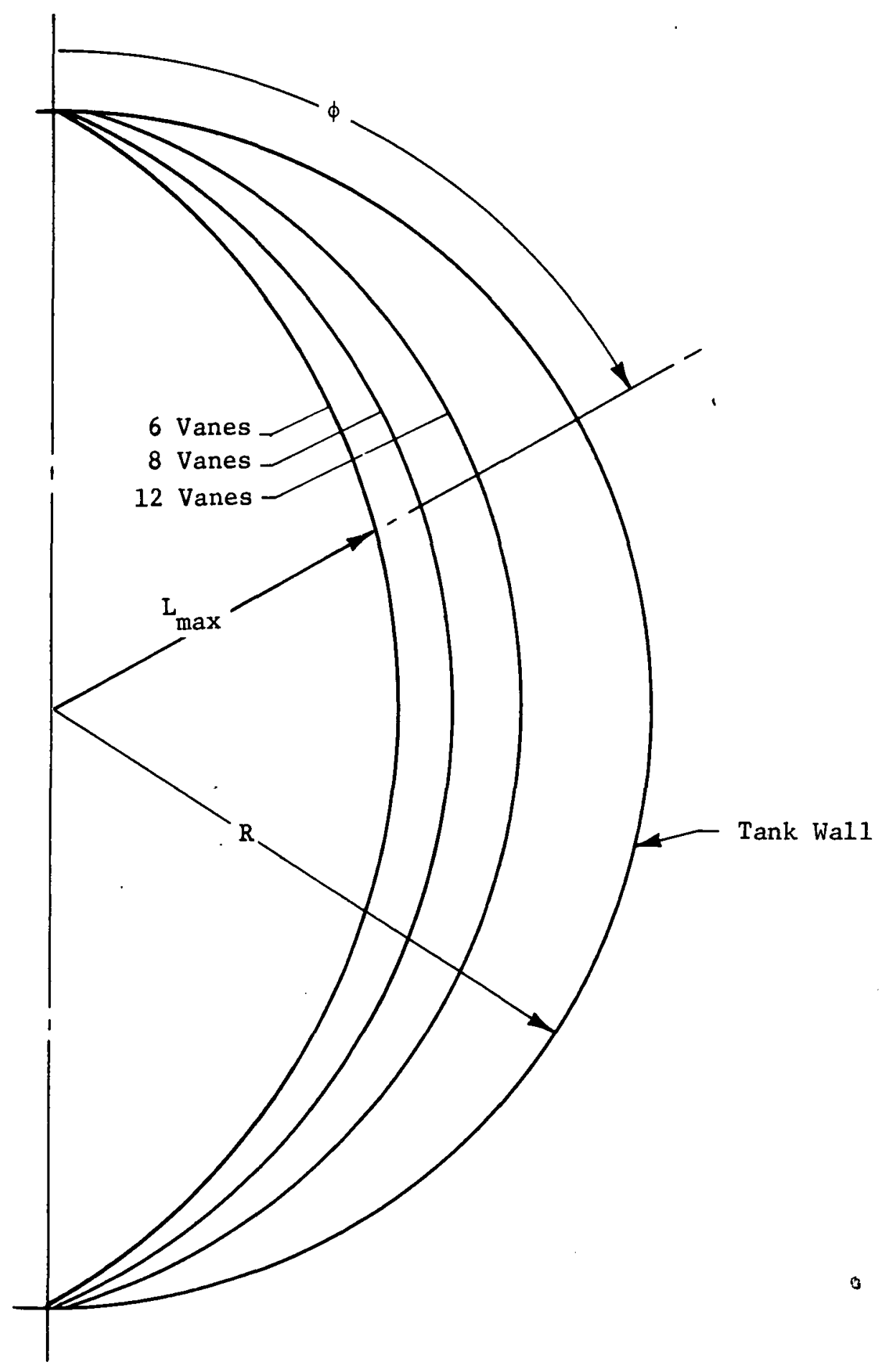

Fig. III-23 Outer Profile Limit 


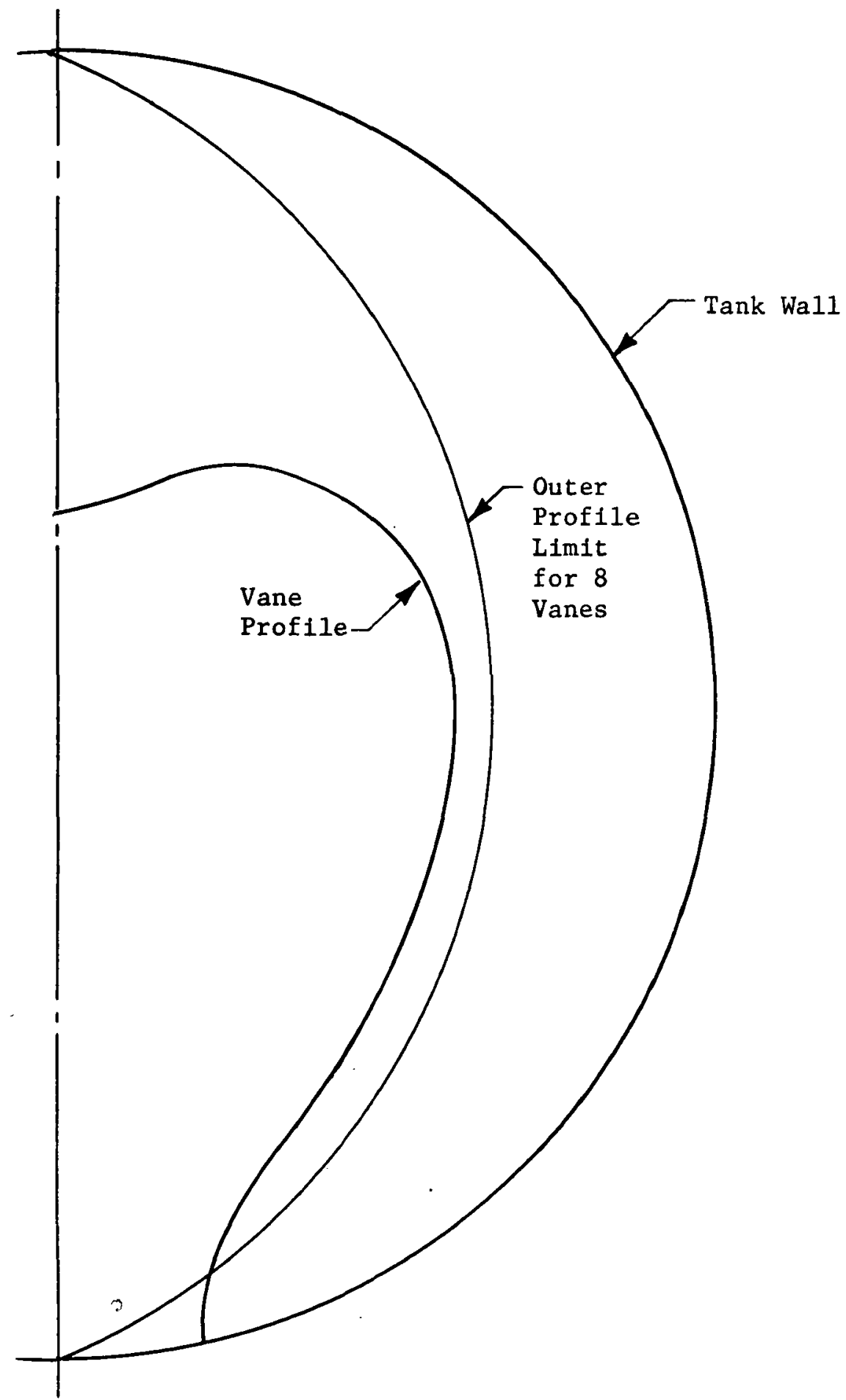

Fig. III-24 Vane Profile at outlet 
The profile of the candidate vane devices in Fig. III-19 and III-20 exceeds the bubble breakup outer limit. For the fuel tank, the vane profile passes through the limit near the tank equator. The corresponding point for the oxidizer tank occurs at about $25^{\circ}$ below the equator. The reason for extending the vane profile beyond the limit is to improve the operation of the device at lower liquid volumes. This is considered highly important in obtaining gasfree liquid expulsion during the terminal phase of the mission. Breakup of the ullage is possible, but not probable. When the mission acceleration environment is considered, it is seen that conditions leading to ullage breakup are so unlikely that this factor should not influence system design for this application. Reasons for this conclusion are discussed in the following paragraphs.

2) Vane Pumping Capability - The capillary forces acting to center the ullage bubble should continue to increase as the bubble is displaced from the centered position. No stable states, other than the centered position, should exist as was found for the standpipe. It is not sufficient to just increase the radius of the vane profile as it progresses from the tank centerline to the outlet. Due to the divergence of the vanes, the capillary pumping effectiveness of the vanes tends to decrease with the distance from the tank centerline.

Vane profiles were analyzed by calculating the capillary forces acting on a bubble of a specified volume as the bubble is displaced from the centered position. The effectiveness of the vanes and the shape of the bubble was approximated, so the force acting on the bubble becomes proportional to the difference in curvature between opposite ends of the bubble. A sketch of the geometry is shown in Fig. III-25. It was assumed that the effective profile determines how the bubble fits within the vanes. Circular arcs of radius $r_{a}, r_{b}$, and $r_{c}$ form the ullage bubble; therefore, the capillary pressure difference across the bubble is:

$$
P_{b}-P_{a}=\sigma\left(\frac{1}{r_{b}}+\frac{1}{r_{c}}\right)-\sigma\left(\frac{1}{r_{a}}-\frac{1}{r_{c}}\right)
$$

or

$$
P_{b}-P_{a}=\sigma\left(\frac{1}{r_{b}}-\frac{1}{r_{a}}\right)
$$



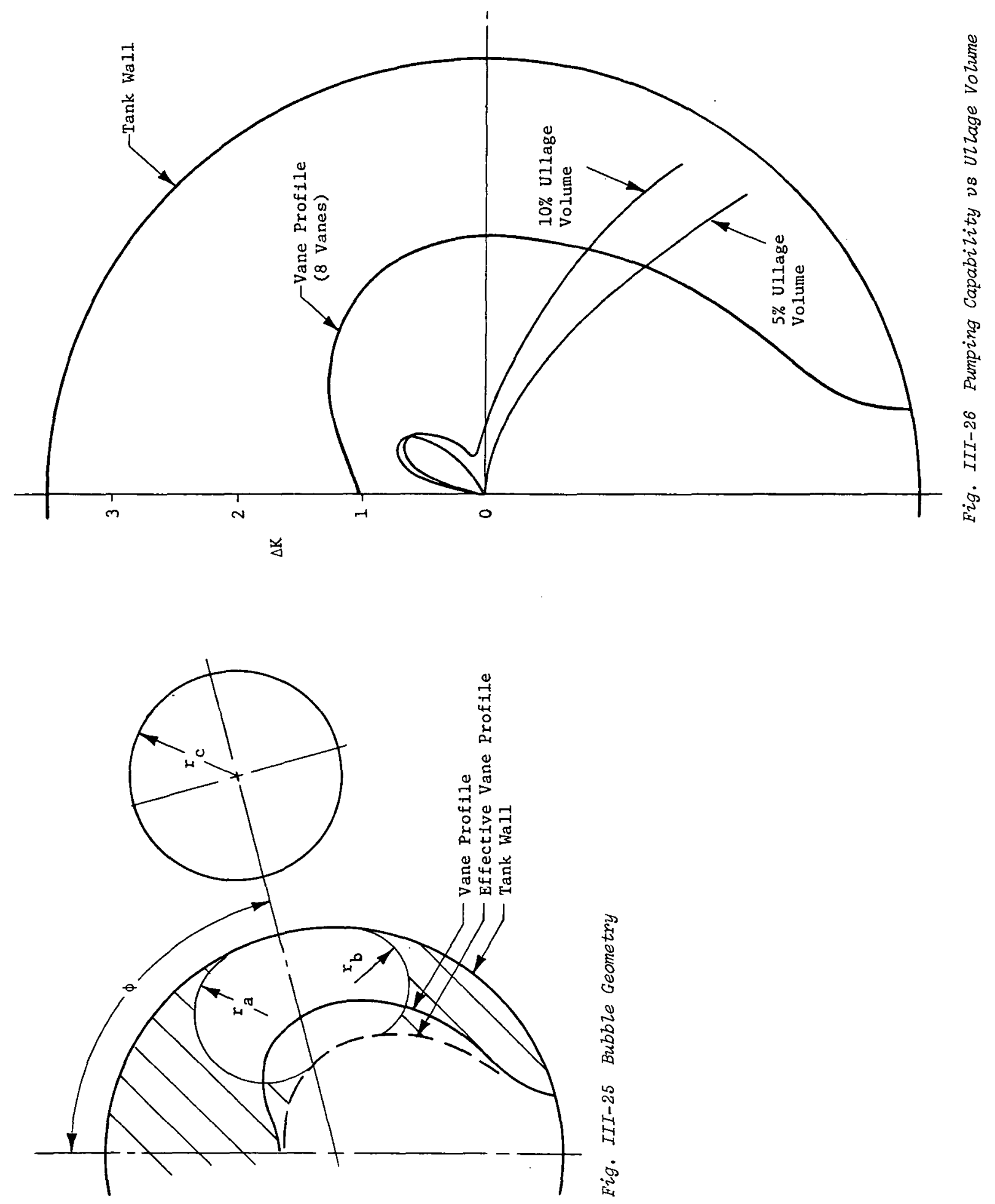

III -36 
Letting $\Delta \mathrm{K}=\frac{1}{\mathrm{r}_{\mathrm{b}}}-\frac{1}{\mathrm{r}_{\mathrm{a}}}$, the difference in curvature, $\Delta \mathrm{K}$, was calculated as a function of the displacement, $\phi$, for a given vane profile, number of vanes, and ullage volume. An example of the results is shown in Fig. III-26. The vane profile shown is for an eight-vane system. The pumping capability, $\Delta \mathrm{K}$, is superimposed on the profile using polar coordinates. $\Delta \mathrm{K}$ is measured radially from the tank center and the displacement is referenced to the center of the bubble.

When the bubble is centered, $\Delta \mathrm{K}$ is zero, and the bubble is in its stable state. As the bubble is displaced $\Delta \mathrm{K}$ increases, indicating the existence of a centering force. The curve for a $5 \%$ ullage shows that this vane profile does have another stable state at a displacement of $80^{\circ}$, at which the value of $\Delta \mathrm{K}$ becomes very small. The effective profile has a region in which the radius decreases slightly. Increasing the ullage to $10 \%$ eliminates this other stable state. There is a region in which the centering force reaches a minimum, but the force is always present. At $10 \%$ ullage, the bubble spans the region in which the profile effectiveness decreases, so the effect is less significant. It can be seen that the profile is least effective in centering the bubble at the minimum ullage volume. Through a process of specifying the effective vane profile and calculating the actual vane profile, the centering capability of the device can be optimized. Vane profiles using this approach for 6-, 8-, and 12-vane devices are shown in Fig. III-27 thru III-29. In each case, the bubble breakup outer limit was used as a design criterion.

When the outer profile limit is exceeded, as it was for the selected vane device profile, the larger vane profile increases the effective profile of the vane. Therefore, a significant increase in the pumping capability of the vane device is obtained by exceeding the outer limit. This is another reason for not using the outer limit as a design criterion.

Vane profiles for a $20 \%$ ullage volume were determined using the same approach. Due to their smaller height, a continuing increase in centering force is easily obtained. 

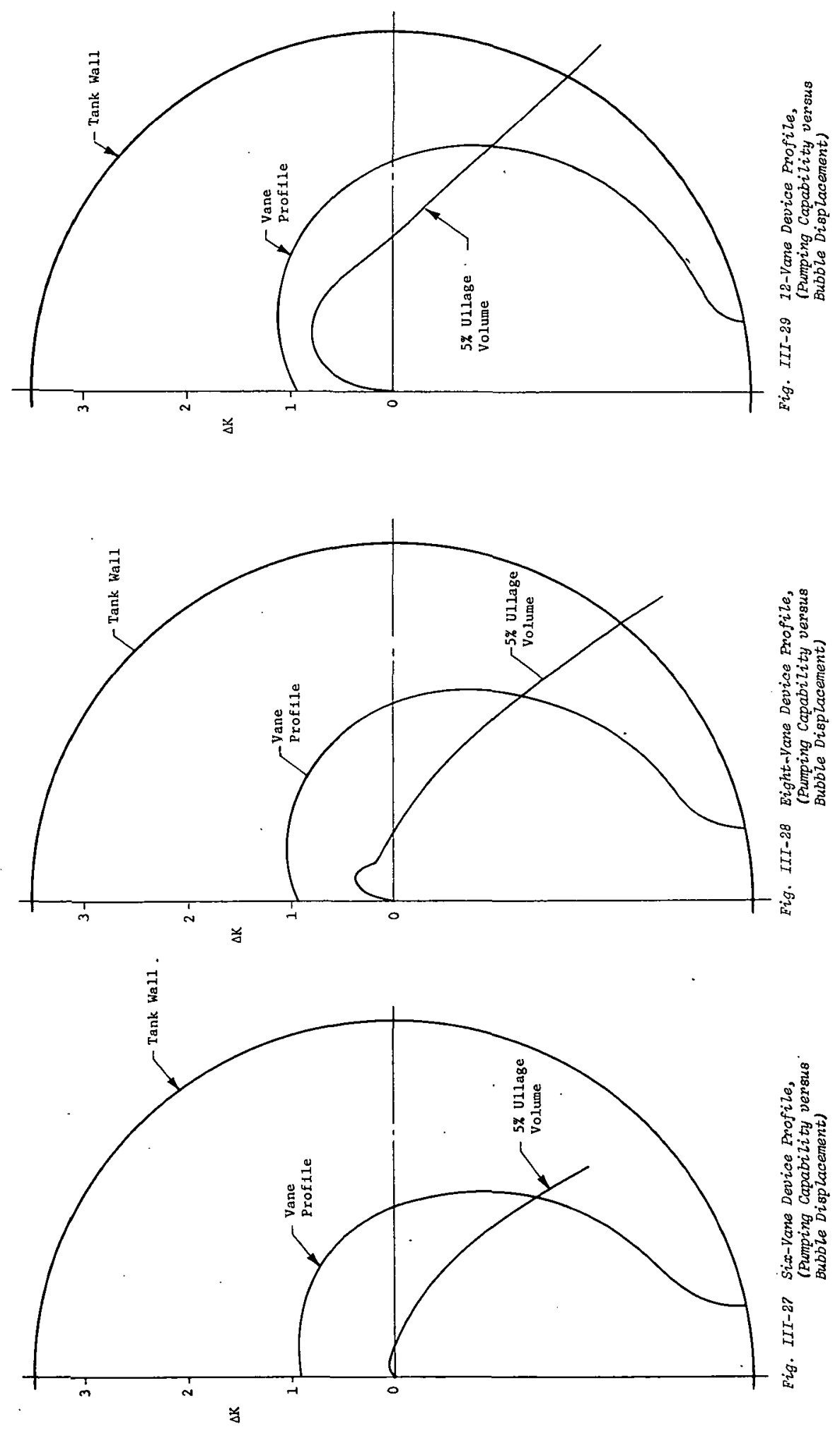
b. Stability - Drop tower tests were used to establish the axial stability of the vane devices. Based on the results, the devices hold a significant amount of liquid over the outlet when axial accelerations equal to and greater than mission accelerations are acting on the device. The results are discussed in detail in Chapter IV.

Axial stability is a function of the interface curvature; the higher the curvature the more stable the interface. A perforated plate is highly stable because the interface at each hole in the plate has a high curvature. On this basis, the relative stability of various vane devices can be determined. In general, vane devices are more stable than the standpipe, but they are less stable than a standpipe with vanes. Liquid within a standpipe is more stable than liquid about a vane device, but the volume of liquid that can be held in the standpipe is limited. When comparing liquid outside a standpipe with that about a vane device, the vane device offers more stability. Considering only vane devices, increasing the number of vanes increases the stability.

Lateral stability depends on the pumping capability of the vane device. As the bubble is laterally displaced, the capillary force increases until it balances the hydrostatic pressure of the liquid. For the given mission, lateral disturbances will be produced by firing the attitude control system (ACS) thrusters and by boom deployment.

Those disturbances produced by the ACS will be considered first. The ACS thrusters operate in a pulsing mode, firing $0.1 \mathrm{sec}$ once every 1 to $4 \mathrm{sec}$ as the spacecraft attitude is being changed. A mechanical analog model was conceived to describe the motion of the ullage bubble due to ACS thrusting. This analog is a springmass system where the spring represents the capillary pumping capability of the acquisition device. As the ullage bubble is. displaced from its centered, equilibrium position, a restoring force, which is proportional to the displacement, acts to recenter the bubble. The mass of the analog is equal to the mass of the liquid being displaced by the bubble motion. For conservatism, no viscous damping was considered. The acceleration produced by the ACS thrusters is acting on the system.

The model was used to analyze the vane devices shown in Fig. III-27 thru III-29. Displacement of the center of the bubble was obtained as a function of time. Using the vane device illustrated in Fig. III-28, the displacement with time shown in Fig. III-30 was obtained. Continuous firing of the thrusters for $15.6 \mathrm{sec}$ will 
move the ullage bubble so that the pressurization/vent line is submerged in liquid, while a $0.1-\mathrm{sec}$ pulse only moves the bubble about $0.001 \mathrm{in}$. These results are typical for any of the vane devices. This analysis indicates that the vane profile does not have to provide large centering forces (the spring constant of the analog) to keep the ullage bubble positioned due to disturbances produced by the ACS. The analysis only considered the effect of a single pulse; continued periodic pulsing is evaluated later in Subsection 8.

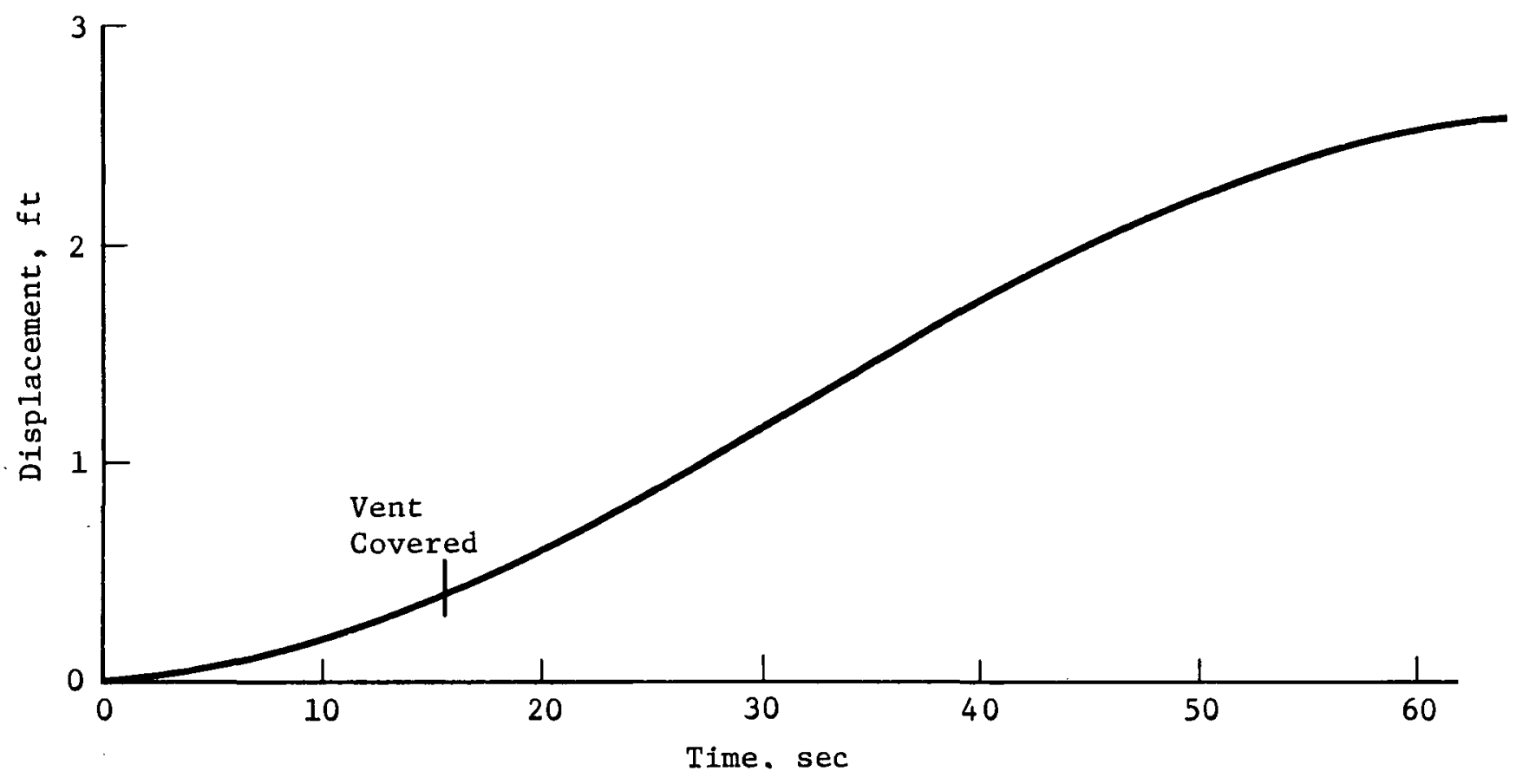

Fig. III-30 UZlage Bubble Displacement vs Time

After the ACS thrusters have set the spacecraft into motion, it continues to rotate at a constant angular rate. A constant angular acceleration would be acting on the liquid as the spacecraft rotates. According to the spacecraft criteria, a lateral acceleration as large as $3 \times 10^{-7} \mathrm{~g}$ could be produced by the spacecraft rotation. This acceleration would not cause any significant liquid displacement and the ullage bubble would remain centered. 
Boom deployment produces disturbances which will last for a period of $45 \mathrm{sec}$. With the magnitude and duration of this disturbance (up to $8 \times 10^{-5} \mathrm{~g}$ for $45 \mathrm{sec}$ ), it is difficult for a vane device to hold the ullage bubble in contact with the vent port. The lateral stability tests confirmed that the bubble will be displaced to the side of the tank for accelerations of that size.

The pumping capability, $\Delta \mathrm{K}$, of the vanes can be used to obtain an approximation of the lateral acceleration necessary to move the bubble away from the vent port. A capillary pressure was calculated using the value of $\Delta \mathrm{K}$ for a bubble displaced to the point at which it is about to lose contact with the vent. An acceleration was then calculated that would produce a hydrostatic pressure across the bubble equal to the capillary pressure. Typical values for this acceleration were on the order of $1 \times 10^{-5} \mathrm{~g}$ for the selected six vanes, $2 \times 10^{-5} \mathrm{~g}$ for eight vanes, and $4 \times 10^{-5} \mathrm{~g}$ for 12 vanes. Therefore, these vane devices would not allow venting during boom deployment.

The possibility of ullage bubble breakup occurring during boom deployment was considered. For bubble breakup to occur with the candidate vane device, the ullage must be displaced so that it is entirely within the region of the vane device that exceeds the outer profile limit (tank outlet region). Breakup of the ullage bubble would then occur as the system reverts to a zero-g environment. If any of the ullage bubble is outside the region in which the profile exceeds to outer limit, there is no way to isolate portions of the bubble within the vanes.

Using the approach described above, the acceleration necessary to displace the bubble to the region of the device in which the profile exceeds the outer profile limit was found to be on the order of $2 \times 10^{-4} \mathrm{~g}$ for the selected six-vane devices. None of the mission disturbances are this large. When the acceleration is removed, the bubble will be pumped back out of the vanes and recentered.

c. Interface Shapes - With all the previously discussed acquisition devices, the interface is completely symmetrical (the interface is the surface of revolution formed by rotating a line about the centerline of the tank) when there is no lateral disturbance acting on the system. Such interfaces can be exactly calculated for any given liquid volume, axial acceleration, and contact angle because the problem is reduced to only two dimensions by the symmetry. Vane devices, however, have three-dimensional interfaces, making the problem of determining the shape of the interface 
very complex. The shape of the interface can be approximated, but techniques for exactly calculating such interfaces have not been developed. Drop tower tests were used to supplement the approximate techniques for determining the shape of the interface.

At low ullage volumes, the interface is a sphere or ellipsoid in low $\mathrm{g}$. In the analysis described above, approximate methods of calculating the interface shape were used to determine the positioning and the stability of the initial ullage volume.

In determining the ability of the vane device to provide a gasfree supply of liquid to the engine, the shape of the interface at low liquid volumes is critical. The ideal acquisition device would position all the liquid directly over the outlet in sort of a dome shaped mass so that the distance from the interface to the outlet was constant. With such a device there would be very little settling or splashing of the liquid as the engine starts. During the critical period in which liquid is flowing to the engine and acceleration is still increasing, the large volume of liquid over the outlet will eliminate suction dip. The interface can be drawn into the outlet if sufficient liquid is not available.

For the vane device, the number of vanes and their profile influence the liquid interface. The curvature of the interface must be the same everywhere, so the device should have its sharpest corners at the outlet. If this is done, more liquid will have to collect at the outlet before the curvature could be reduced to a value equal to the curvature elsewhere.

The greater the number of vanes, the sharper the junction between the vanes at the central post. This will cause liquid to fill the vanes in preference to the base of the device. Various numbers of vanes were tested in the drop tower to demonstrate this fact. With a 12-vane device, most of the liquid is within the vanes, leaving very little directly at the outlet. A six-vane device will still center the ullage bubble, but only a small amount of liquid will be drawn up into the vanes.

The profile of the vane works in conjunction with the number of vanes to determine where the liquid will position with respect to the device. A profile that becomes broader as it nears the outlet will tend to orient the liquid toward the outlet. This is the reason for the profile of the candidate devices, as opposed to the profile shown in Fig. III-27. This effect was also demonstrated in the drop tower tests. 
In comparison to the ideal acquisition device discussed above, the vane device is less effective in orienting small liquid volumes. A tall device is required for centering the ullage bubble, but a short device would orient small liquid volumes in the preferential location over the outlet. Because of the height of the device, some liquid will be held up in the vanes, but this was minimized by using only six vanes and the selected profile. When the engine starts, this liquid will settle, causing some splashing at the surface. Liquid already oriented at the outlet will not be distributed, so it will isolate the outlet from the action of the settling liquid. The possibility of ingesting gas bubbles generated by the settling is discussed in Subsection 8 of this analysis.

Standpipe With Vanes

This Category $C$ device is a combination of the standpipe and vane system. The configuration of the candidate device is shown in Fig. III-31 for the oxidizer tank and in Fig. III-32 for the fuel tank. The number and profile of the vanes are the same as the vane device discussed in the previous subsection. Performance and operation of this device is similar to that for the vane device. only the variations will be discussed here.

a. Standpipe - A tapered standpipe, which is smaller than the basic standpipe device, is used. The internal volume of the standpipe is $0.4 \%$ of the tank volume in the oxidizer tank and $0.9 \%$ in the fuel tank. Liquid outside the standpipe feeds directly to an annular outlet that surrounds the base of the standpipe. Large openings in the base allow liquid to enter and leave the standpipe.

The standpipe provides a highly stable reservoir of liquid that supplements the liquid held about the device. At all liquid volumes greater than about $5 \%$, the standpipe will be completely full of liquid since the curvature of the interface inside the standpipe is less than the curvature of the interface outside the standpipe. Equilibrium is only established at these volumes after the interface inside the standpipe becomes stuck. During the critical engine start phase of operation, the standpipe will become unstable and supply an additional volume of liquid directly to the outlet. 


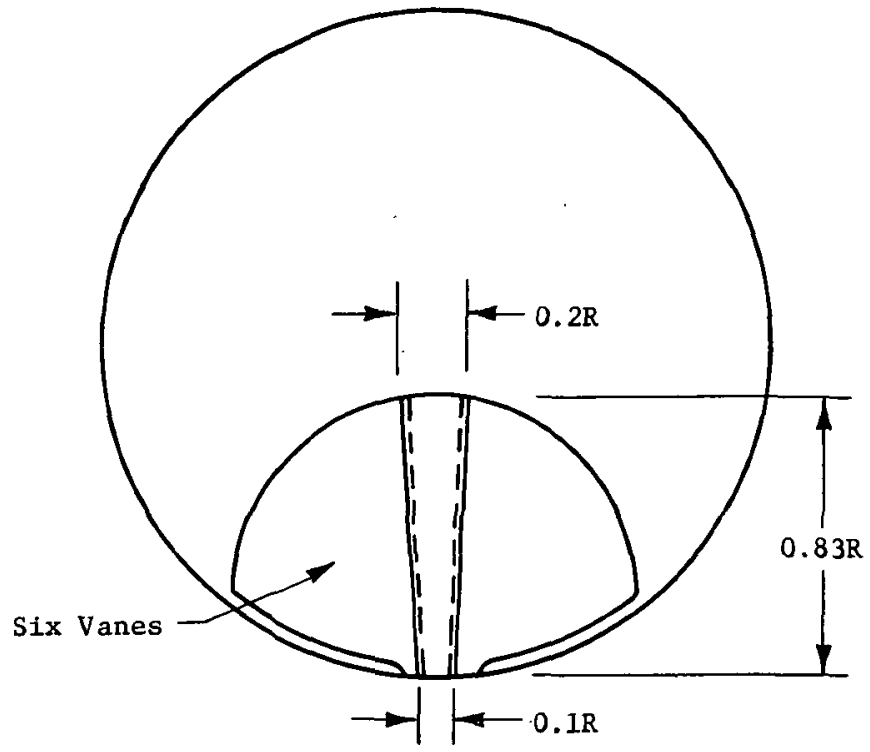

Fig, III-3I Standpipe with Vanes for Oxidizer Tank

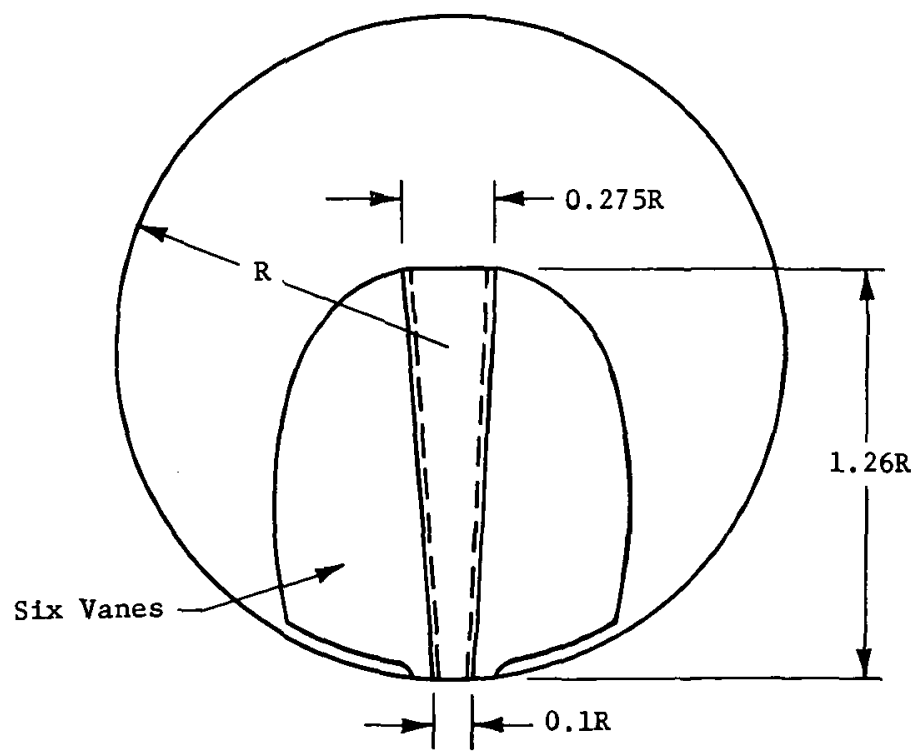

Fig. III-32 Standpipe with Vanes for Fuel Tank 
At liquid volumes less than $5 \%$, the static interface shape will be reached with a partially full standpipe. Due to the taper of the standpipe, a decrease in the volume of liquid outside the standpipe will produce a proportional decrease in volume inside the standpipe because the curvatures of the interfaces must equalize. Since the outlet is located outside the standpipe, it is not desirable to have a standpipe that holds most of the liquid inside.

b. Communication Channels - If some or all of the liquid is moved away from a Category $C$ acquisition device, communication channels are provided for return of this liquid to the device. All five of the Category $\mathrm{C}$ devices will be considered in this analysis.

If the interface of the liquid that has been moved away is in contact with the device, the channels serve no purpose. But, when that interface does not touch the device or come into contact with the liquid about the device, the channels will interconnect the displaced liquid and the liquid about the device. One requirement for the initiation of liquid return to the device is that the displaced liquid be in contact with at least one channel.

A capillary pumping pressure provides the driving force to cause the flow of liquid along the channels. It is determined, as follows. Under zero $g$, the curvature, $K$, of the interface is the same at any point on the surface. $K$ is related to the principal radii of the surface, $r_{1}$ and $r_{2}$ :

$\mathrm{K}=\frac{1}{\mathrm{r}_{1}}+\frac{1}{\mathrm{r}_{2}}$

To simplify calculations, a dimensionless curvature will be used:

$\mathrm{K}=\mathrm{R}\left(\frac{1}{\mathrm{r}_{1}}+\frac{1}{\mathrm{r}_{2}}\right)$,

where $R$ is the radius of the tank. At any point on the surface, the difference in pressure between the gas and the liquid is given by:

$\mathrm{P}_{\mathrm{g}}-\mathrm{P}_{\ell}=\frac{\sigma \mathrm{K}}{\mathrm{R}}$

where

$\mathrm{P}_{\mathrm{g}}=$ pressure of the gas,

$\mathrm{P}_{\ell}=$ pressure of the liquid,

$\sigma=$ surface tension. 
If there are two separate volumes of liquid, identified by subscripts 1 and 2, the pressure in each is a function of their respective curvatures:

$P_{g}-P_{\ell_{1}}=\frac{\sigma}{R} K_{1}, P_{g}-P_{\ell_{2}}=\frac{\sigma}{R} K_{2}$,

or

$\mathrm{P}_{\ell_{1}}=\mathrm{P}_{\mathrm{g}}-\frac{\sigma}{\mathrm{R}} \mathrm{K}_{1}, \mathrm{P}_{\ell_{2}}=\mathrm{P}_{\mathrm{g}}-\frac{\sigma}{\mathrm{R}} \mathrm{K}_{2}$.

Therefore,

$P_{\ell_{1}}-P_{\ell_{2}}=\left(P_{g}-\frac{\sigma}{R} K_{l}\right)-\left(P_{g}-\frac{\sigma}{R} K_{2}\right)$

and

$P_{\ell_{1}}-P_{\ell_{2}}=\frac{\sigma}{R}\left(K_{2}-K_{1}\right)$

The difference in pressure between the two liquid volumes is directly proportional to the difference between the curvatures. Volume 1 will be at a higher pressure than volume 2 if the curvature of volume 1 is less than volume 2 .

The curvature of a given volume of 1iquid under zero $g$ is influenced by contact angle and the geometry of the surface containing the liquid. Liquid located in a sharp corner will have a higher curvature than liquid on a smooth surface. A flat interface has a curvature of zero.

Consider a displaced volume of liquid in contact with a communication channel. Liquid in the sharp channel has a curvature much larger than that of the bulk liquid volume. Therefore, the pres-' sure of the liquid in the channel is less than that of the displaced liquid, and liquid will flow into and rapidly fill the channel.

In the drop tower tests, filling of the channels occurred in approximately $2 \mathrm{sec}$. Scaling this time on a basis of the ratio of $\frac{D \mu}{\sigma}(D=$ tank diameter, $\mu=$ viscosity, and $\sigma=$ surface tension $)$, the time required to fill the channels would be about $25 \mathrm{sec}$ for the Flox tank and $35 \mathrm{sec}$ for the MMH tank. The time required to fill the channels can be neglected in comparison to the rate at which the liquid is pumped after the channels are full. This pumping time is discussed later. 
Once the channel has filled, pressure is still not in equilibrium, so the liquid will flow about the sharp corners of the acquisition device. As the area about the device fills with liquid, the curvature of that volume will decrease, reducing the pumping pressure. Pumping of the liquid continues until the curvature, and therefore the pressure, if uniform everywhere.

The magnitude of the pressure difference, which can exist when liquid has been displaced away from the device, was calculated for the post device. Since the fingers of the post-with-fingers device have very little effect on the curvature, the same results would be obtained for that device. All these calculations were accomplished for zero contact angle and zero g. Percent liquid volume is plotted versus the dimensionless curvature in Fig. III-33 for liquid volumes located at the top and bottom of the tank. Only volumes that do not contact each other are of interest, so the height at which the interface contacts the wall, $h_{\text {wall }}$, has also been plotted versus curvature. Based on the coordinate system shown in Fig. III-33, $h_{\text {wall }}$ for the liquid at the top of the tank will be greater than $h_{\text {wall }}$ for the liquid at the bottom of the tank if the two interfaces are not in contact ( $\mathrm{h}_{\text {wall }}$ was made dimensionless by dividing by the tank radius).

At the top of the tank, the interface will be in dontact with the device if the liquid volume is approximately $25 \%$ ( $\mathrm{h}_{\text {wall }}$ approaches zero). The curvature approaches 2.0 at small volumes because both principal radii approach the tank radius. At the bottom of the tank, the acquisition device causes the curvature to increase at small liquid volumes. When $h_{\text {wall }}$ is less than 1.0 , the curvature increases rapidly as the volume decreases.

Use of the curves in Fig. III-33 is best explained by an example. Assume there is a volume of liquid equal to $5 \%$ of the tank volume at the top of the tank and a volume equal to $0.5 \%$ of the tank volume at the tank bottom about the device. The interface of the liquid at the top of the tank contacts the wall at $h_{\text {wall }}=0.32$ and its curvature is 2.04. The interface of the liquid about the device contacts the tank wall at $h_{\text {wall }}=0.19$ and its curvature is 2.36. The two interfaces are separated by $0.13 \mathrm{R}$. Therefore, the pressure difference between the two liquid volumes is:

$$
\mathrm{P}_{\text {top }}-\mathrm{P}_{\text {bottom }}=\frac{\sigma}{\mathrm{R}}(2.36-2.04)=0.32 \frac{\sigma}{\mathrm{R}} .
$$


(ITEM

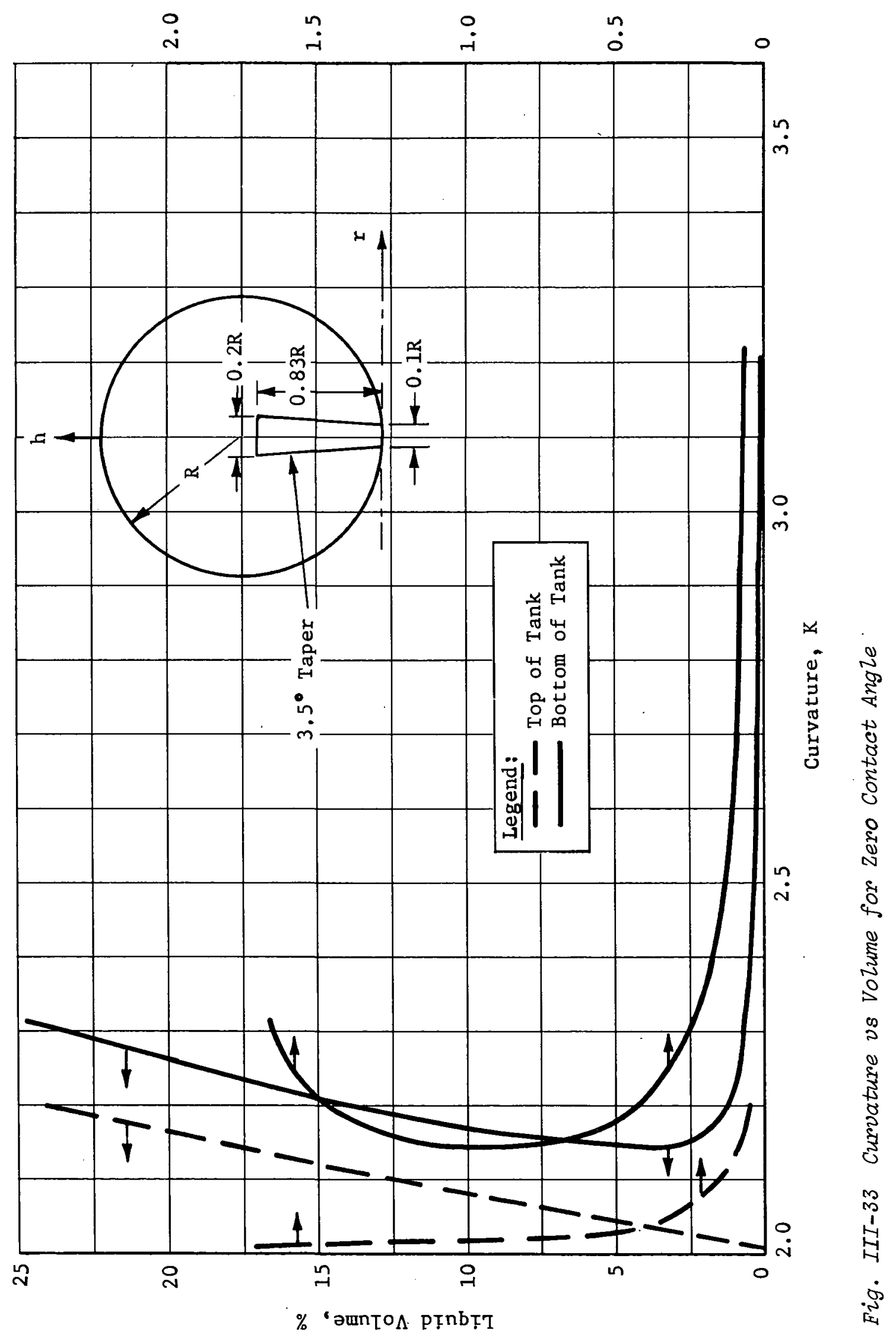


The value of $\frac{\sigma}{R}$ for each propellant and tank combination of interest is presented in Table III-3.

Tabze III-3 $\frac{\sigma}{R}$ for Each Propezzant

\begin{tabular}{|l|l|l|l|}
\hline \multirow{2}{*}{ Propellant } & $\sigma \frac{1 b_{\mathrm{f}}}{\mathrm{ft}}$ & Tank R (ft) & $\frac{\sigma}{\mathrm{R}}$ (psi) \\
\hline Flox & $0.927 \times 10^{-3}$ & 1.47 & $4.38 \times 10^{-6}$ \\
$\mathrm{M} \mathrm{HH}$ & $2.35 \times 10^{-3}$ & 1.20 & $1.36 \times 10^{-5}$ \\
$\mathrm{~F}_{2}$ & $0.912 \times 10^{-3}$ & 1.35 & $4.68 \times 10^{-6}$ \\
$\mathrm{~N}_{2} \mathrm{H}_{4}$ & $4.62 \times 10^{-3}$ & 1.35 & $2.37 \times 10^{-5}$ \\
\hline
\end{tabular}

Because Flox has the smallest $\frac{\sigma}{R}$ and therefore the smallest pumping pressure in comparison with the three other propellants, it was evaluated as the worst-case situation.

$P_{\text {top }}-P_{\text {bottom }}=(0.32)\left(4.38 \times 10^{-6}\right)=1.4 \times 10^{-6} \mathrm{psi}$.

This pressure difference is acting to move more liquid toward the device.

A closer study of the curves in Fig. III-33 indicates that the curvature of the interface about the device is always greater than the curvature of the interface at the top of the tank, for nonintersecting interfaces. This implies that the pressure of the liquid about the device will always be less than the pressure at the top of the tank. In fact, the pressure difference reaches a minimum and can not be any less. This minimum occurs when the volume of liquid about the device is about $4 \%$; at the top of the tank, the volume would have to be less than $1 \%$ or the interfaces will intersect. For these volumes, the curvature difference is 0.14 , so the minimum pressure difference for Flox is $6.2 \times 10^{-7}$ psi.

Another plot of curvature versus liquid volume was calculated for the same geometry, but with a contact angle of $5^{\circ}$ (Fig. III-34). The most noticeable difference, in comparison to the zero contact angle case, is the variation of the curvature of the liquid at the top of the tank at small volumes. Below $2.5 \%$ 1iquid 


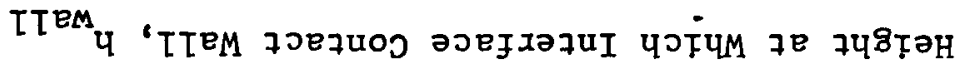

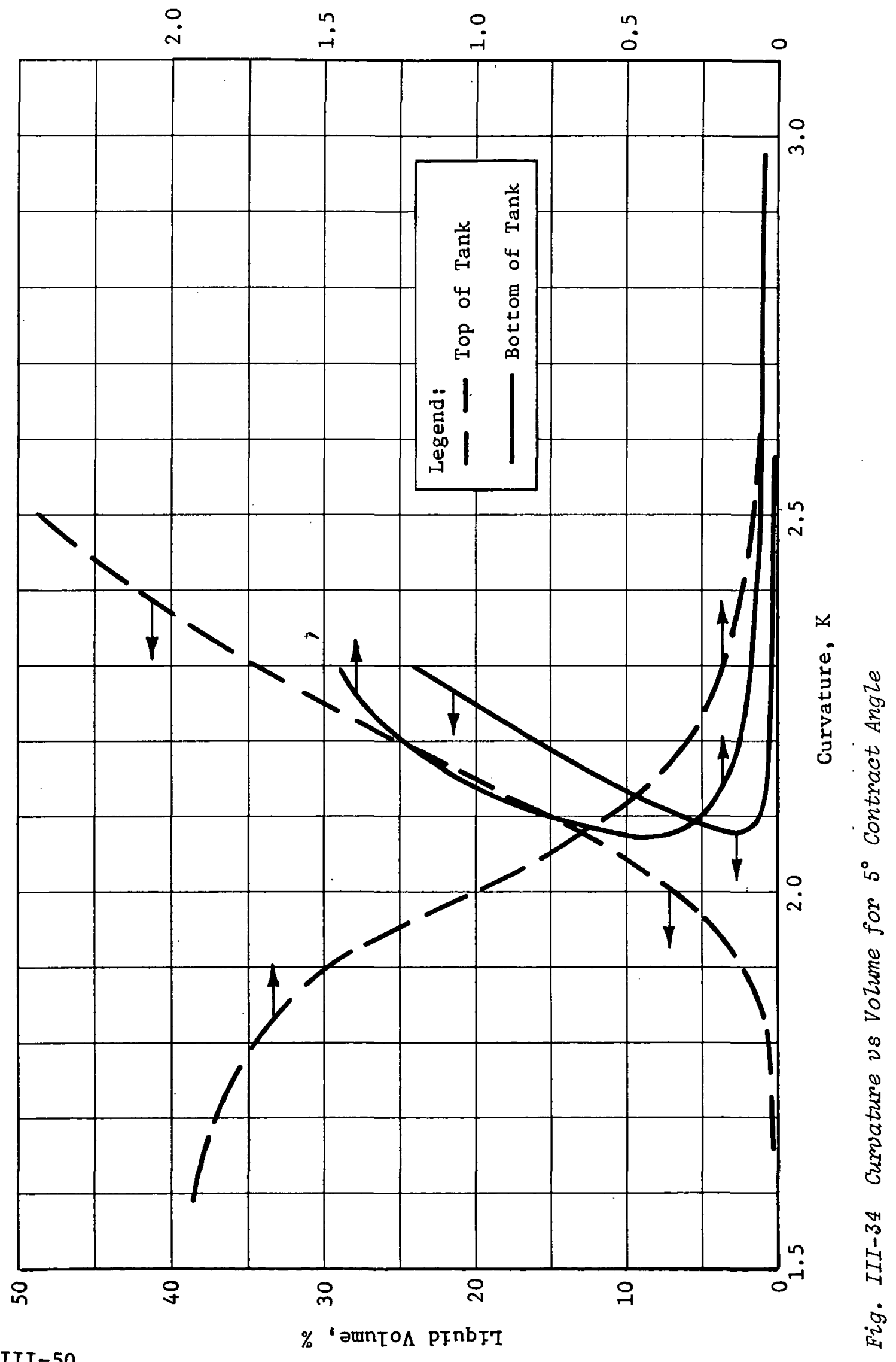


volume at the tank top, the curvature decreases rapidly.* Liquid about the device has a minimum curvature at $2.5 \%$ liquid volume and $h_{\text {wall }}=0.45$ as compared to $4 \%$ volume and $h_{\text {wall }}=1.0$ for zero contact angle.

The most significant difference between the $5^{\circ}$ contact angle curve and the $0^{\circ}$ curve is that, under certain conditions, the pumping pressure can be zero or can be acting to move liquid away from the device to the top of the tank with a $5^{\circ}$ contact angle. This occurs for curvatures between 2.07 and 2.73. Volumes at the top of the tank must be between $12.5 \%$ and $59 \%$, and the volume at the bottom of the tank must be between $0.17 \%$ and $2.5 \%$. The following examples explain the implications of this contact angle effect:

1) If there was $22.4 \%$ liquid in the tank and a disturbance of some sort had moved $21.8 \%$ away from the device, no pumping pressure would be available to move liquid along the communication channels to the device. The curvature would be the same at the top and bottom of the tank $(K=2.17)$. This situation is illustrated in Fig. III-35.

2) A worst-case condition would exist if there was $57 \% 1$ iquid in the tank with 55\% located away from the device. The curvature is greater at the top of the tank, so liquid would be pumped away from the device until the curvatures were equal. At this point, only $0.2 \%$ liquid would remain about the device.

This same phenomena does not occur at zero contact angle because the interfaces intersect before the curvature at the top of the tank becomes equal to the curvature at the bottom of the tank. Further investigation of the post device showed that a favorable capillary pressure differential will exist only if the contact angle is less than or equal to $2^{\circ}$.

*At $0.001 \%$ volume the interface is flat, because it intersects the tank wall at $5^{\circ}$. A flat interface has a curvature of zero. At smaller volumes the curvature becomes negative (convex interface). 


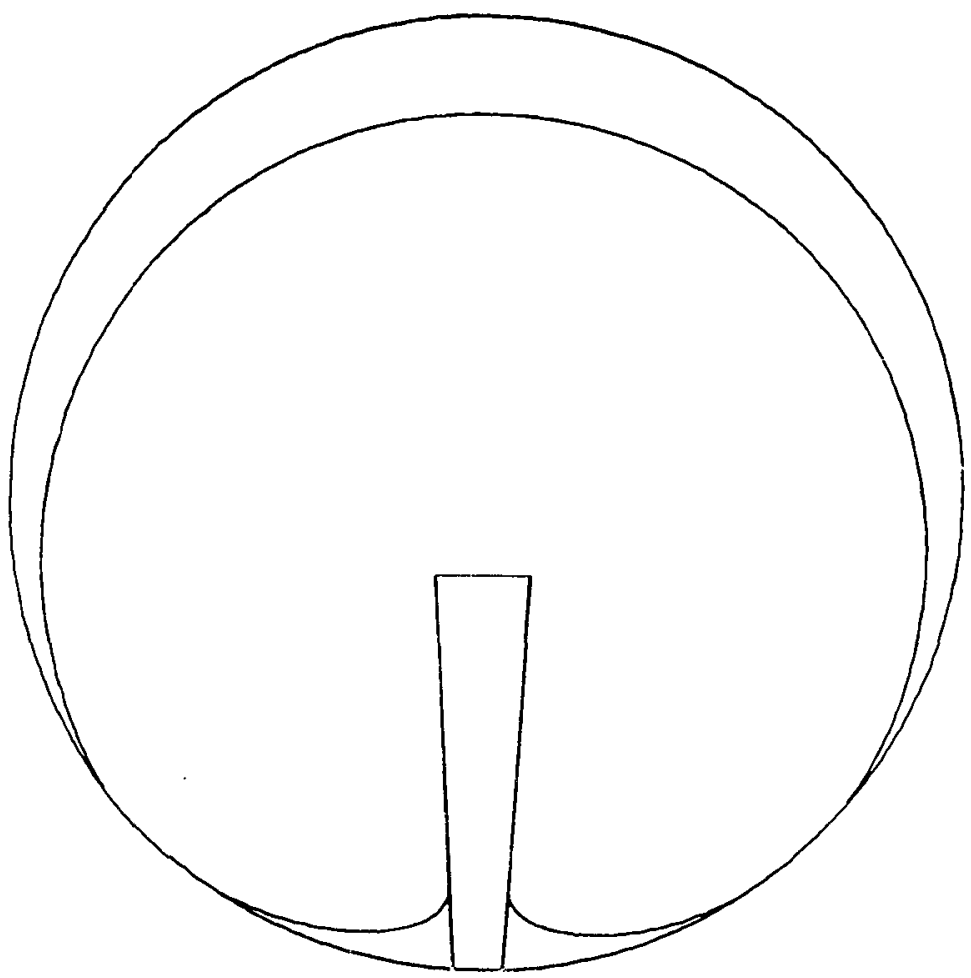

Fig. III-35 Equilibrium Configuration for $5^{\circ}$ Contact Angle

Changes to the configuration of the acquisition device will alter the curvature versus volume relationship. Increasing the taper of the post to $7^{\circ}$, double that of the post in Fig. III-33, would increase the curvature of the interface about the device. Displaced liquid would always be at a lower pressure than liquid about the device at contact angles up to $3^{\circ}$. Another modification, the addition of small fins at the base of the post, would increase the curvature at low liquid volumes.

A device that has a greater effect on the curvature, such as a standpipe or vane device, would be suitable for use with liquids having much larger contact angles. In Fig. III-36, the standpipe and vaned devices have been added to the Fig. III-34 situation. Al1 of the curves are for a $5^{\circ}$ contact angle. The standpipe, with a diameter of 0.4 times the tank radius, will always have functional communication channels at this contact angle, i.e., the pressure about the device will always be less than the pressure of any displaced liquid. It can be seen that the vaned device will provide a much higher capillary pumping pressure. The curvature of the interface about the standpipe with vanes would be essentially the same as it is for the vane device. At low liquid volumes the standpipe would increase the curvature somewhat. 


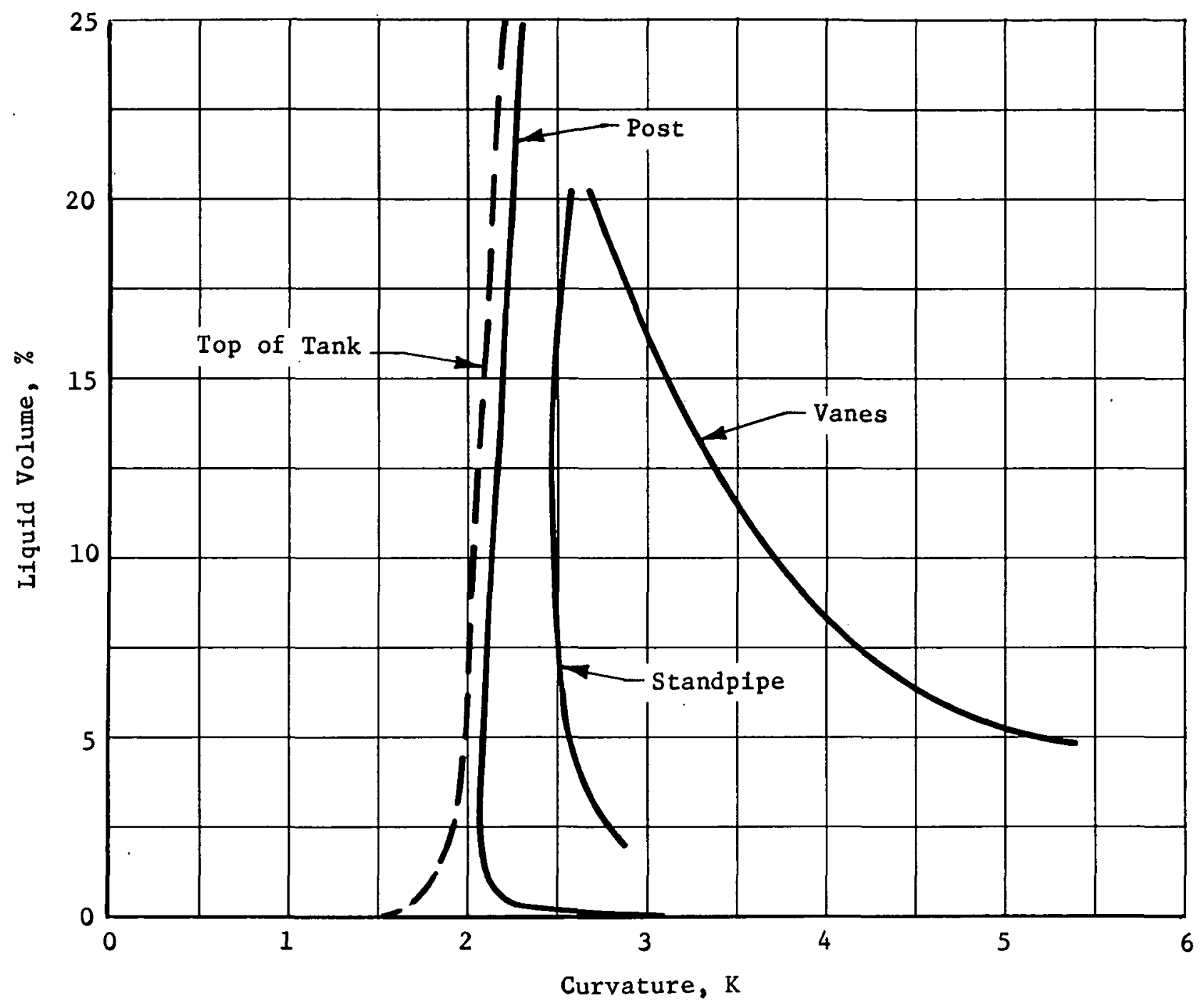

Fig. III-36 Comparison of Curvature for Three Devices at $5^{\circ}$ Contact Angle 
This analysis indicates that unless contact angles very near to zero degrees can be guaranteed, communication channels will not always be functional with devices that do not significantly alter the curvature of the interface about the device. Conversely, a system, such as a standpipe with vanes, which has a high curvature will function properly over a wide range of contact angles.

With the pressure differential between the ends of the communication channel established, the rate at which liquid will flow along the channel can be determined. A good representation of the flow can be obtained by using low Reynolds number, creeping flow equations. For a channel with an equilateral triangle cross section, the flowrate is given by the following equation from Reference III-3:

$\mathrm{Q}=\frac{\sqrt{3} \Delta \mathrm{P} \mathrm{b}^{4}}{320 \mu \mathrm{L}}$

where

$Q=$ Volumetric flowrate,

$\Delta \mathrm{P}=$ differential pressure,

$b=$ length of side of triangle,

$\mu=$ viscosity,

$L=$ length of channel.

The maximum length of a communication channel in a spherical tank is $L=\pi R$, where $R$ is tank radius. Using the previously developed relationship for the pressure differential, $\Delta \mathrm{P}=\frac{\sigma}{\mathrm{R}} \Delta \mathrm{K}$, the time required, $t$, to transfer a fraction of the tank liquid volume, $f$, is given by

$\tau=\frac{2420 f \mu R^{5}}{\Delta \mathrm{K} \sigma b^{4}}$

The Flox tank, which presents the worst case, was evaluated assuming a single triangular channel with a 1-in. side. Using the minimum $\Delta \mathrm{K}$ of the post device shown in Fig. III-33, the time required to transfer $5 \%$ of the tank volume would be $180 \mathrm{hr}$. If all the liquid were displaced from the post, returning $5 \%$ should be adequate to provide for engine start and settling of the remainder of the 
propellant. A vaned device, assuming a $\Delta \mathrm{K}$ of 2.0 based on Fig. III-36, would reduce the time to $13 \mathrm{hr}$. Using four channels instead of one would reduce the time to abour $3 \mathrm{hr}$. These results indicate that return of a significant amount of displaced liquid within a reasonable length of time is possible.

8. General Device Performance

The following four analyses apply to most of the candidate devices.

a. Propelzant Slosh due to ACS Operation - The ACS thrusters are used to maintain and change the spacecraft inertial orientation, and to unload the momentum wheels. Unloading the momentum wheels produces a $10^{-4} \mathrm{~g}$ axial acceleration that assists the operation of the acquisition device because it tends to settle the propellants over the outlet. ACS operation that changes the spacecraft orientation, however, produces a lateral acceleration of the propellant tanks tending to move liquid away from the device and induce slosh. This occurs during the trajectory correction maneuver (TCM) when the ACS orients the spacecraft for the main engine burn and, following the burn, when the ACS orients the spacecraft to reacquire celestial reference.

If excessive slosh due to thruster pulsing should occur, liquid would be displaced from the device. There is only a period of a few minutes between the termination of the ACS maneuvers and ignition of the main engine, so return of a large quantity of liquid to the device with communication channels might not be feasible. Also, continued sloshing of the liquid could disturb the inertial reference system due to the forces applied to the tanks.

A typical sequence for a TCM is shown in Fig. III-37, which was constructed from information presented in Reference III-4. At $M+45$ minutes, the guidance system switches to inertial reference and remains in this mode for a period up to 160 minutes. Six minutes after reacquisition of the sun and Canopus on the sensors, the guidance system switches back to celestial reference. During the TCM, the ACS roll and yaw maneuvers and the main engine burns are accomplished.

The ACS thrusters have a constant pulse width of $0.1 \mathrm{sec}$ and rotate the spacecraft by pulsing at a rate that usually falls between 0.25 and 1.0 pulses per sec. These pulses will produce lateral accelerations on the order of $3 \times 10^{-5}$ to $7 \times 10^{-5} \mathrm{~g}$. Lateral slosh of a significant magnitude will be induced if the driving frequency is near the harmonic oscillation frequency of the liquid-tank system. 


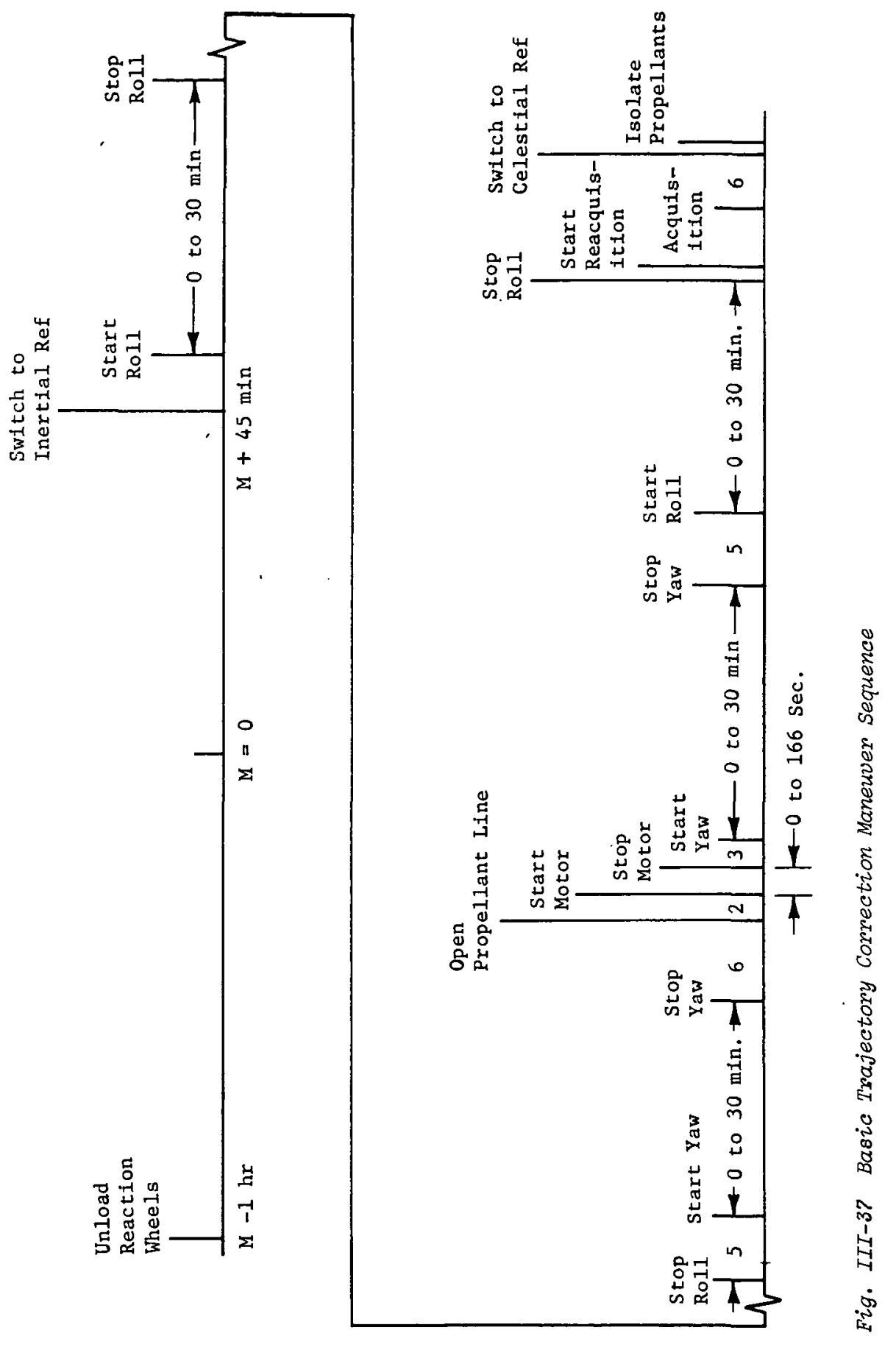

III -56 
The first mode harmonic frequency parameter for lateral slosh in a spherical tank is plotted versus Bond number for various tank volumes in Fig. III-38 (Ref III-5). Exciting the system at the first mode frequency produces slosh with a greater magnitude than excitation at higher modes. The Bond number is based on the axial acceleration acting on the tank. The dimensionless frequency, $\omega$, used in Fig. III-38, is defined as:

$\omega^{2}=\frac{\bar{\omega}^{2}}{(1+\mathrm{BO}) \frac{\sigma}{\rho \mathrm{R}^{3}}}$

where

$\bar{\omega}=$ dimensional applied frequency (radians/sec),

Bo $=$ axial Bond number,

$\sigma=$ surface tension,

$\rho=$ density,

$\mathrm{R}=$ tank radius.

Typical values for $\omega^{2}$ were calculated for each of the four propellants and their respective tanks during ACS operation. The results are listed in Table III-4. An $\bar{\omega}$ of $0.25 \mathrm{cps}$, a typical thruster firing frequency, was used for the calculations.

Table III-4 Value of $\omega^{2}$ for Each Propezlant Tank

\begin{tabular}{|l|l|l|}
\hline Propellant Tank & $\omega^{2}$ & Bo \\
\hline Flox & $2.26 \times 10^{4}$ & 0.064 \\
$\mathrm{MMH}$ & $0.31 \times 10^{4}$ & 0.010 \\
$\mathrm{~F}_{2}$ & $1.84 \times 10^{4}$ & 0.056 \\
$\mathrm{~N}_{2} \mathrm{H}_{4}$ & $0.26 \times 10^{4}$ & 0.008 \\
\hline
\end{tabular}

Comparing the value of $\omega^{2}$ in Table III-4 with the curves for the harmonic $\omega^{2}$ in Fig. III-38 shows that the frequency imposed by thruster firing is much greater than the frequency at which first mode harmonic slosh would be induced. Even the fifth mode harmonic frequency, which is greater than the first mode, is still much less than the frequency due to thruster firing. Based on this comparison, it can be concluded that the ACS orientation maneuvers will not produce slosh of significant magnitude. 


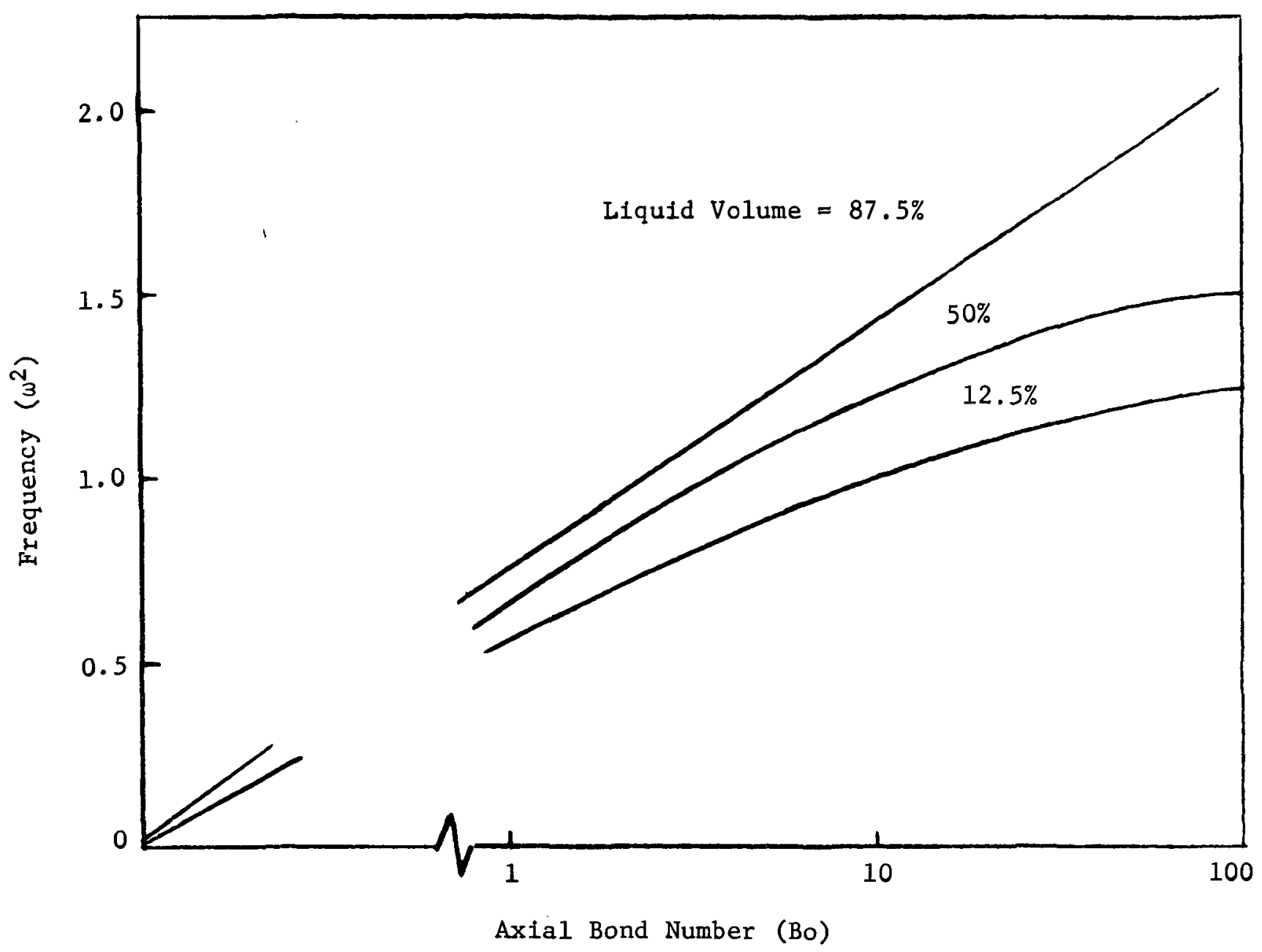

Fig. III-38 First Mode Harmonic Frequency for Spherical Tank (Ref III-5) 
Following the roll and yaw maneuvers, there are periods of 5 to 6 minutes before the next event is accomplished. Some pulsing of the thrusters may be required to maintain the inertial attitude during these periods. The frequency of these pulses can be much lower and therefore closer to the harmonic frequency. It is doubtful that significant slosh would be induced though, because the thrusters would be firing randomly and continued firing near the harmonic frequency would not occur. In addition, a few shortduration pulses would not supply enough energy to produce significant slosh.

Another factor to be considered when evaluating slosh in spherical tanks is their natural damping capability. Motion of the liquid at volumes both above and below $50 \%$ is dissipated by the curved walls of the tank (Ref III-ó). Furthermore, many of the prope1lant acquisition devices being considered have a configuration that will act as slosh suppression baffles.

b. Vehicle Center of Gravity - During thrust startup transients, excessive vehicle rotation (sufficient to lose control of the vehicle) could develop due to an excessive shift in the vehicle cg. Therefore, the ability of the propellant acquisition system to control propellant location (minimize movement) is an important criterion for evaluating performance. A simple computer program was written to calculate the vehicle $\mathrm{cg}$ as a function of component masses and locations. With this program, the effects of the propellant mass distribution provided by the acquisition device on the vehicle cg may be evaluated.

To determine the magnitude of any cg shift, it was necessary to establish the initial cg location for the loaded vehicle. A reference configuration for a Jupiter Orbiter Spacecraft was provided by JPL (JPL Drawing 10041593). Figure III-39 illustrates the reference vehicle propellant tank envelope established by the retracted positions of the RTG boom and the scan science package, the payload shroud, the electronics compartment, and the engine. It was found that the baseline propellant tanks were too large to be contained within the reference envelope in both the in-1ine and side-by-side tank configurations. Therefore, the envelope length was either increased or decreased as required by each particular configuration. The clearances between the electronics compartment and the propellant tank, between the two propellant tanks, and between the propellant tank and engine head, shown on the Fig. III-39 reference drawing, were used for all tank arrangements. 


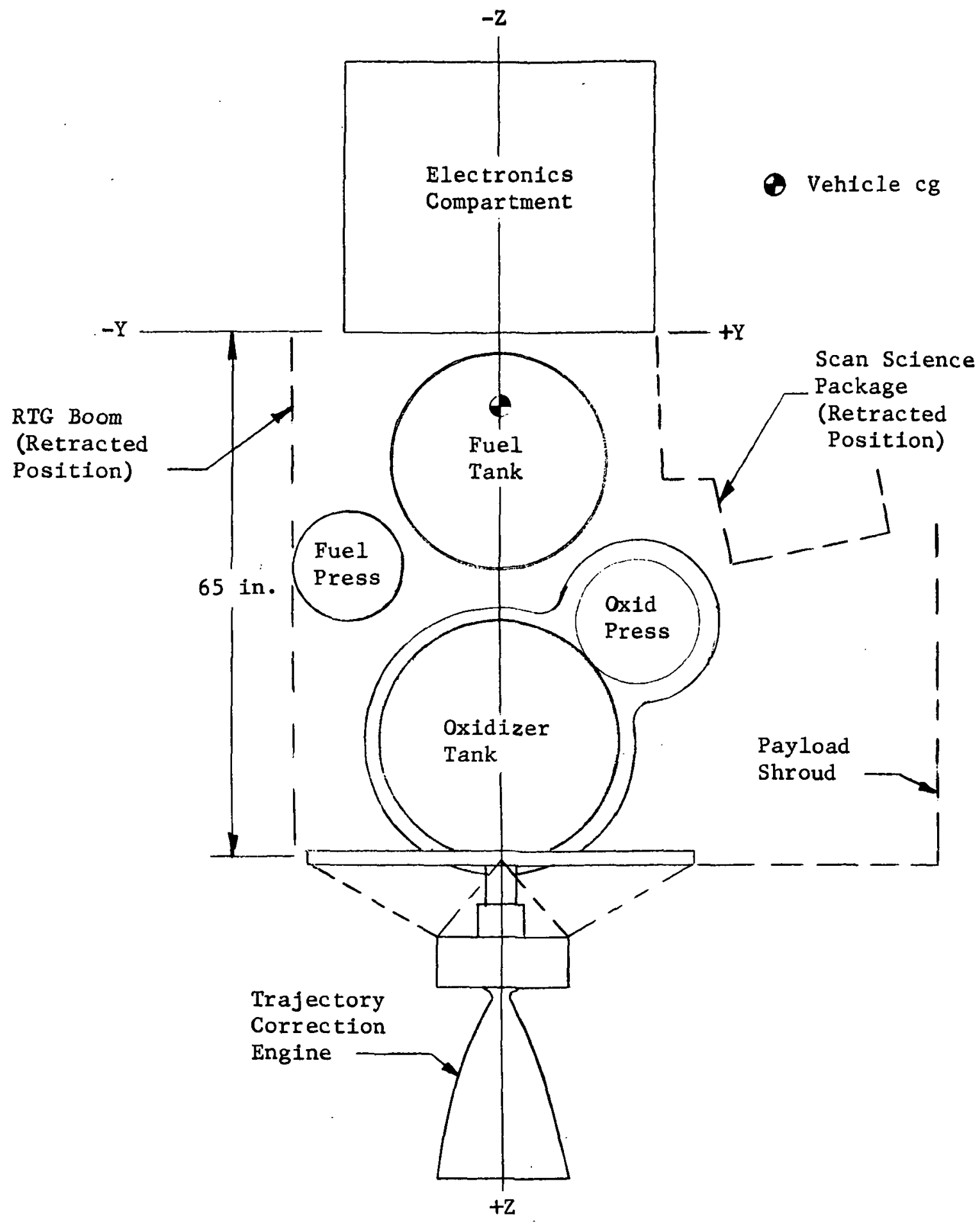

Fig. III-39 Reference Spacecraft Propellant Tank Envelope (per JPL Drowing 10041593) 
Figures III-40 and III-4I present the in-line tank arrangements for Flox/MMH and $\mathrm{F}_{2} / \mathrm{N}_{2} \mathrm{H}_{4}$ propellant combinations, respectively. The Flox/MMH system requires lengthening the reference envelope by $8 \mathrm{in}$. while the $\mathrm{F}_{2} / \mathrm{N}_{2} \mathrm{H}_{4}$ system requires a 9-in. increase. The tanks in both systems are mounted on the $Z$ axis with the pressurant spheres in the $Y-Z$ plane. In the side-by-side tank arrangement, the propellant tanks are positioned in the $X-Z$ plane to avoid interference with the retracted RTG boom and scan science package. Figures III-42 and III-43 illustrate the side-by-side tank orientations for the Flox/MMH and $\mathrm{F}_{2} / \mathrm{N}_{2} \mathrm{H}_{4}$ propellant combinations, respectively. The envelope for the Flox/MMH system is 23 1n. shorter than the reference envelope while that for the $\mathrm{F}_{2} / \mathrm{N}_{2} \mathrm{H}_{4}$ system is 26 in. shorter.

The vehicle $\mathrm{cg}$ was calculated for each of the tankage arrangements shown in Fig. III-40 thru III-43. The results together with the cg location for the reference vehicle are tabulated in Table III-5.

Table III-5 Center of Gravity Coordinates

\begin{tabular}{|ll|c|c|c|}
\hline \multirow{2}{*}{ System } & \multirow{2}{*}{ Tank Arrangement } & \multicolumn{3}{|c|}{ Coordinates, in. } \\
\cline { 3 - 5 } & $\mathrm{X}$ & $\mathrm{Y}$ & $\mathrm{Z}$ \\
\hline Reference & In-line & 0 & 0 & 9.0 \\
Flox $/ \mathrm{MMH}$ & In-line & -0.09 & 0.05 & 17.4 \\
$\mathrm{~F}_{2} / \mathrm{N}_{2} \mathrm{H}_{4}$ & In-line & -0.09 & -0.06 & 15.45 \\
$\mathrm{Flox}_{\mathrm{N}} / \mathrm{MMH}$ & Side-by-side & 0.02 & 0.05 & 3.96 \\
$\mathrm{~F}_{2} / \mathrm{N}_{2} \mathrm{H}_{4}$ & Side-by-side & -0.15 & -0.05 & 3.04 \\
\hline
\end{tabular}

With no propellant acquisition device in the tanks, the liquid can move freely about the tank, and the largest variation in the location of the spacecraft $\mathrm{cg}$ would be expected. This condition was analyzed for the Flox/MMH propellant combination.

The in-line tank configuration was considered first. The cg of the dry spacecraft is located at $-0.17,0.09,-6.48 \mathrm{in}$. using the $X, Y, Z$ coordinate system of Fig. III-39. Therefore, a lateral acceleration acting in the $+X$ direction, so as to move the liquid along the $-X$ axis, would cause the largest lateral shift in the center of gravity. A typical lateral acceleration of $7 \times 10^{-5} \mathrm{~g}$ was used for the calculation of the interface shape and then the $\mathrm{cg}$ of the liquid in each tank was calculated. A sketch of this situation is shown in Fig. III-44. Finally, the spacecraft $\mathrm{cg}$ was calculated from the liquid mass and $\mathrm{cg}$ prior to each of the first six engine burns. After the sixth burn, the mass of liquid remaining is too small to have an effect comparable to that of the larger liquid volumes. The results are compiled in Table III-6. Prior to the fourth burn, the largest shift occurs with the cg moving 2 in. along the $-\mathrm{X}$ axis. 

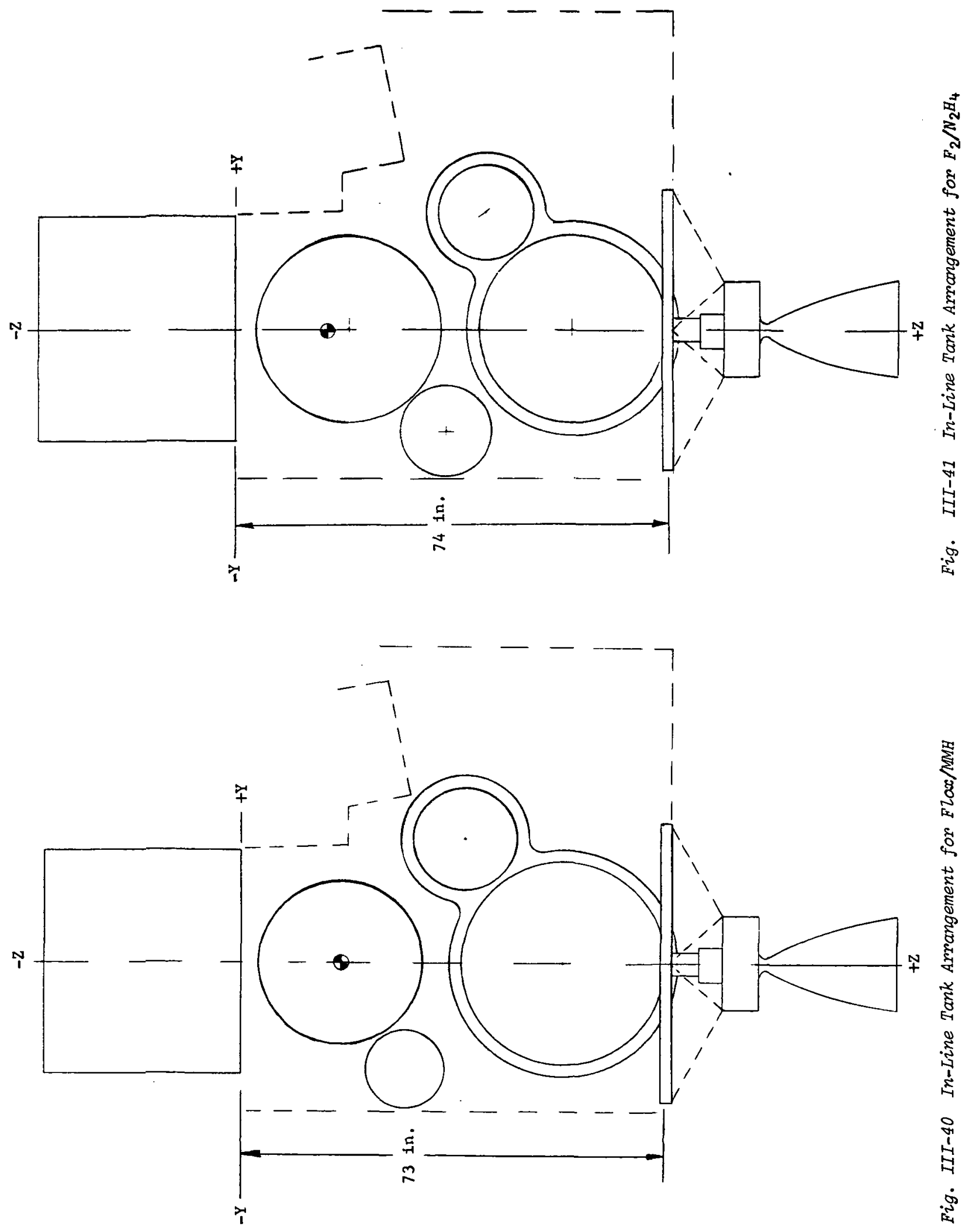

 


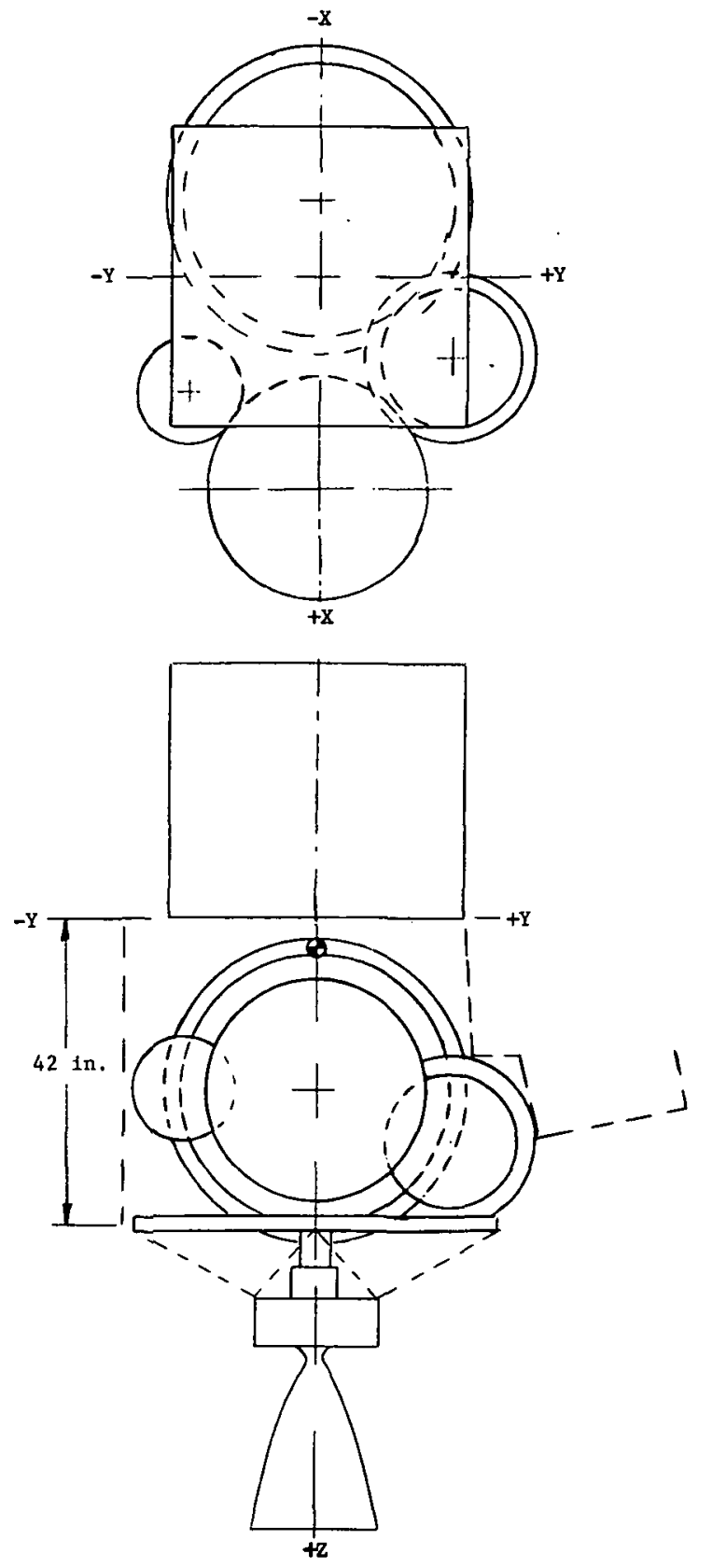

Fig. III-42 Side-by-Side Tank Arrangement for Flax/MMB

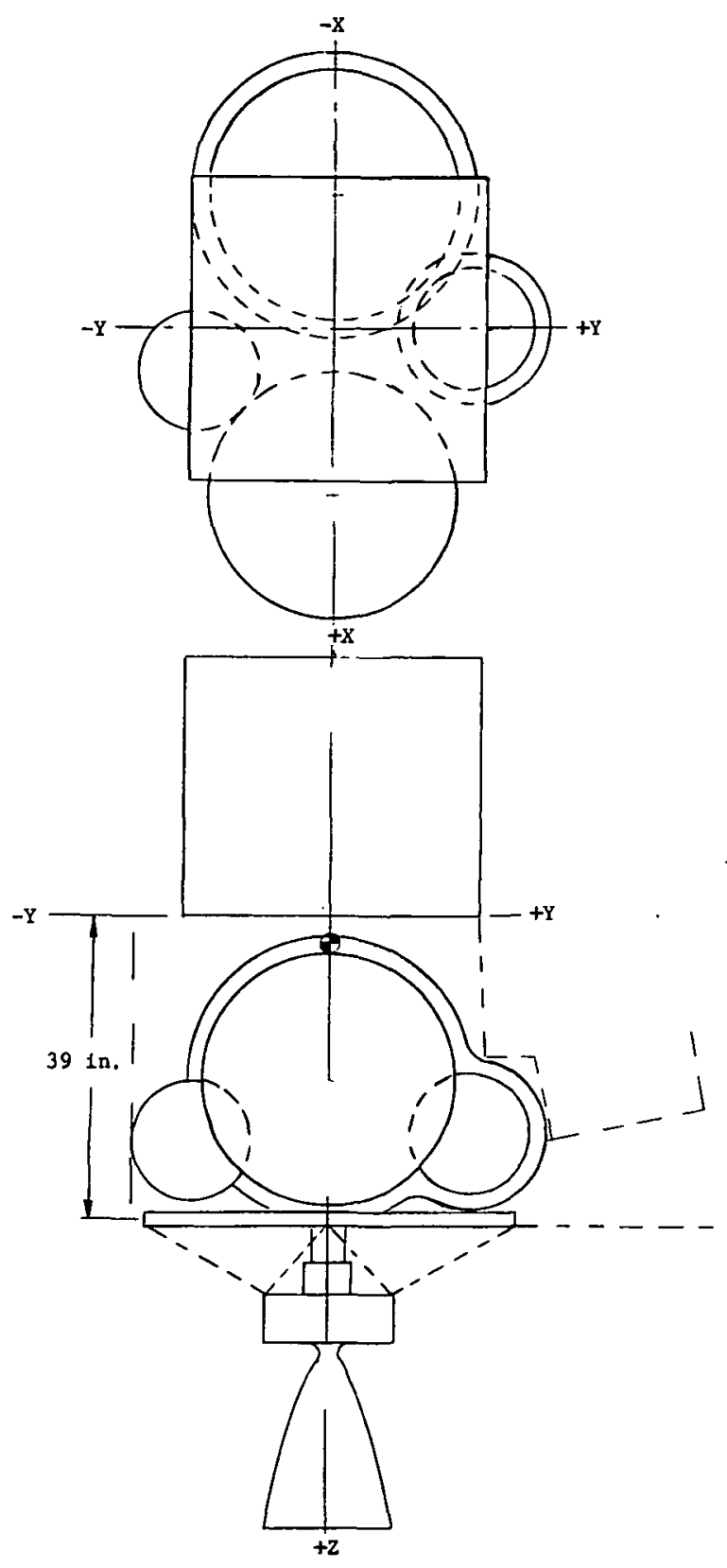

Fig. III-43 Side-by-Side Tank Arrangement for $\mathrm{F}_{2} / \mathrm{N}_{2} \mathrm{~B}_{4}$ 


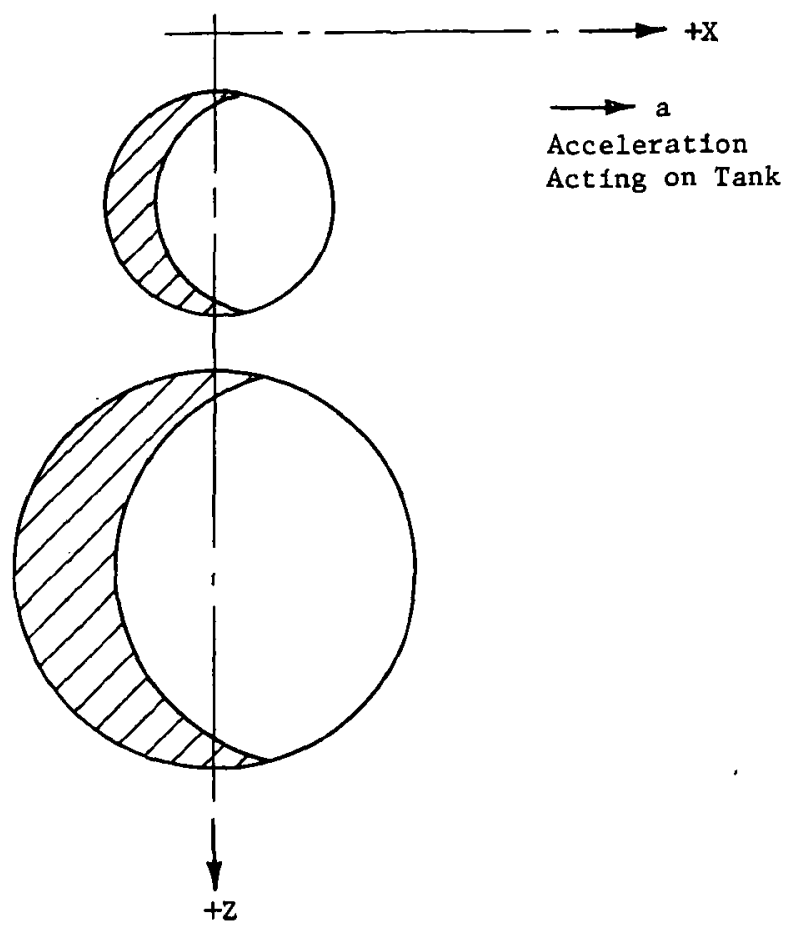

Fig. III-44 In-Line Tank Arrangement og Shift

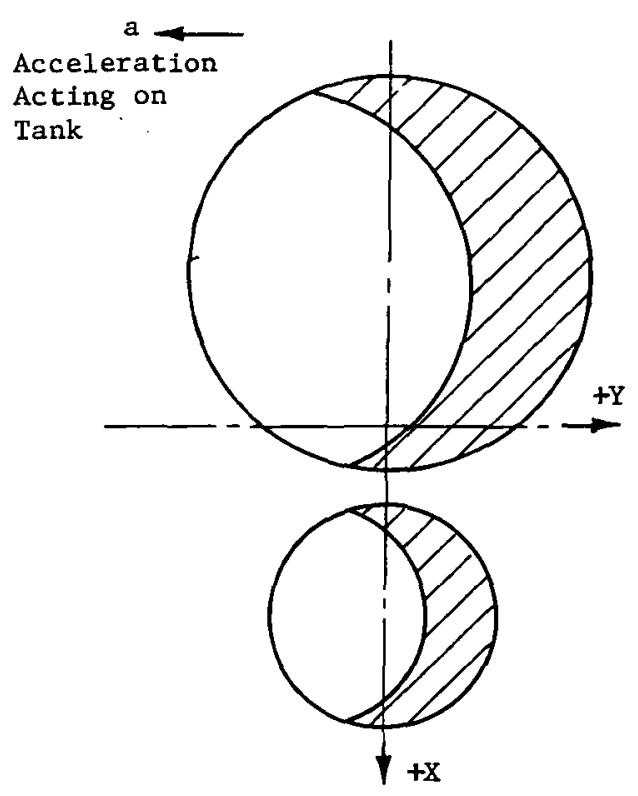

(a) Acceleration Along Y Axis

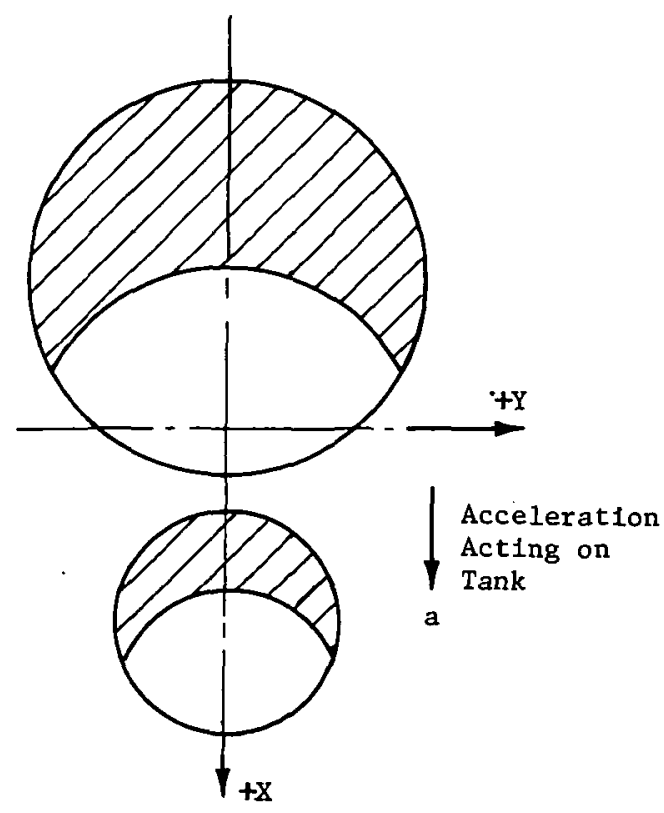

(b) Acceleration Along $\mathrm{X}$ Axis

Fig. III-45 Side-by-Side Tank Arrangement cg Shift

III-64 
Table III-6 Location of $\mathrm{cg}$, In-Line Tanks

\begin{tabular}{|l|l|l|l|}
\hline \multirow{2}{*}{} & \multicolumn{3}{|l|}{ Location, in. } \\
\cline { 2 - 4 } & $\mathrm{X}$ & $\mathrm{Y}$ & $\mathrm{Z}$ \\
\hline Dry & -0.17 & 0.09 & -6.48 \\
Before & & & \\
Burn No.: & & & \\
1 & -1.05 & 0.05 & 16.91 \\
2 & -1.08 & 0.05 & 16.75 \\
3 & -1.13 & 0.05 & 16.56 \\
4 & -2.02 & 0.06 & 9.00 \\
5 & -1.89 & 0.07 & 4.31 \\
6 & -1.09 & 0.08 & -2.19 \\
\hline
\end{tabular}

A similar analysis was accomplished for the side-by-side tank configuration where the dry tanks have a cg located at $0.09,0.10$, $-12.05 \mathrm{in.}$ The lateral acceleration was assumed to be acting in the $-Y$ direction so as to move the liquid in both tanks in the $+Y$ direction. This situation is depicted in Fig. III-45(a). The results, presented in Table III-7, show that the largest shift again occurs prior to the fourth burn when the cg moves 2 in.

Table III-7 Location of cg, Side-by-Side Tanks

\begin{tabular}{|l|l|l|l|}
\hline \multirow{2}{*}{} & \multicolumn{3}{|l|}{ Location, in. } \\
\cline { 2 - 4 } & $\mathrm{X}$ & $\mathrm{Y}$ & $\mathrm{Z}$ \\
\hline Dry & 0.09 & 0.10 & -12.05 \\
Before & & & \\
Burn No.: & &. & \\
1 & 0.02 & 1.01 & 3.64 \\
2 & 0.02 & 1.04 & 3.52 \\
3 & 0.02 & 1.09 & 3.40 \\
4 & 0.04 & 1.97 & -1.67 \\
5 & 0.05 & 1.83 & -4.81 \\
6 & 0.07 & 1.03 & -9.17 \\
\hline
\end{tabular}


Another situation was checked to ensure that it would not cause a larger cg shift. Liquid was moved in the $-\mathrm{X}$ direction as shown in Fig. III-45(b). Prior to the fourth burn, the $\mathrm{cg}$ is located at $-1.86,0.07,-1.67$ in.; thus, a 1.86 -in. shift in $\mathrm{cg}$ from the centerline would occur under these conditions.

All of the candidate propellant acquisition devices will hold some liquid along the $\mathrm{Z}$ axis so a smaller $\mathrm{cg}$ shift would occur. A device such as the vanes would hold more propellant along the centerline of the tank than one like the post and would provide a lower $\mathrm{cg}$ shift. In the previous bare-tank calculations, it was assumed that the lateral acceleration was constant and that a static interface would be established. In reality, all the lateral disturbances are short duration and will usually be acting in conjunction with axial accelerations. Therefore, any shifts in vehicle cg will be considerably less than 2 in. with acquisition devices in the tank. The worst-case 2-in. cg shift translates into a $1.5^{\circ}$ gimbal requirement for in-line tanks and a $2^{\circ}$ gimbal requirement for side-by-side tanks. This engine gimbal requirement is well within the engine gimbal capability of $9^{\circ}$.

The next item of interest was to determine if the engine gimbal rate was sufficient to keep up with the rate at which the cg can shift. When the spacecraft engine starts, the cg can travel from its low-g, ACS-perturbed position to the tank centerline with some overshoot and oscillation. A pendulum model was used to describe this motion. The pendulum arm was fixed at 2 in., i.e., the maximum cg displacement, and the spacecraft mass was located at the end of the arm. The pendulum path then represents the path followed by the spacecraft $\mathrm{cg}$ as the liquid settles. The period of this pendulum, under the acceleration of the spacecraft engine, is $0.8 \mathrm{sec}$. The time required for the pendulum to move from its extreme position to its neutral position is one-fourth of the period, or $0.2 \mathrm{sec}$. In other words, a lateral change in cg location of 2 in. occurs in $0.2 \mathrm{sec}$ based on this model. Viscous damping would increase the period, so this is a conservative estimate of the rate of travel. As discussed previously, a 2-in. cg travel corresponds to a $2^{\circ}$ maximum rotation about the gimbal axis. Therefore, the maximum rate of travel of the $\mathrm{cg}$ is $10 \mathrm{deg} / \mathrm{sec}$. A typical guidance/actuator system is capable of responding at a rate of $20 \mathrm{deg} / \mathrm{sec}$ (Ref III -4 ), so this very conservative cg travel rate of $10 \mathrm{deg} / \mathrm{sec}$ is acceptable. 
c. Bubble Ingestion - Usually, the ullage gas forms a single bubble that can be oriented away from the outlet by the propellant acquisition device. Under certain conditions, some breakup of the ullage could occur, allowing small bubbles to be present in the liquid. The most likely cause of these bubbles is the splashing of the liquid during settling. For all the propellants, the settling Bond number is on the order of $10^{4}$ when the spacecraft engine is firing. This indicates that splashing of the liquid will occur during the turbulent settling process. Bubbles will form and could be drawn into the outlet along with the outflowing propellant.

Other, less likely causes of bubble formation are slosh and reorientation of the liquid due to ACS operation. The acquisition device has very little effect on the positioning of any bubbles that are smaller than the capillary dimensions of the device. Therefore, bubbles formed due to these effects could be positioned almost anywhere within the liquid. Under zero $g$, the bubbles will assume a static equilibrium position within the liquid and remain there until the tank is accelerated.

As the tank is accelerated, a buoyancy force is applied to the bubble causing it to rise at a rate dependent on the drag of the liquid. A dimensionless parameter, $M$, determines how the $r$ ise rate varies with bubble radius for a given liquid (Ref III-7):

$M=\frac{a \mu^{4}}{\rho \sigma^{3}}$

where

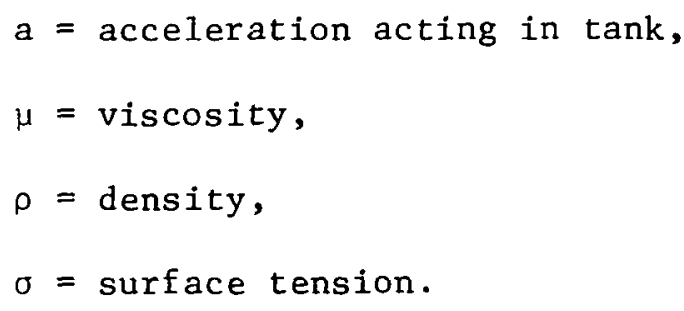

The minimum acceleration produced by the spacecraft engine of $0.19 \mathrm{~g}$ was used in these calculations. The values of $\mathrm{M}$ for Flox and MMH are $1.5 \times 10^{-12}$ and $2.4 \times 10^{-15}$, respectively. For liquids with a value of $M$ less than $10^{-8}$, the rise velocity at first increases rapidly as the bubble radius increases. The velocity achieves a maximum, falls to a minimum, and then gradually rises again. This variation can be divided into two regimes as 
shown in Fig. III-46. The rise velocity is calculated from the following equations:

1st Regime - $U_{b}=\frac{1}{5} \frac{r_{b}^{2} \rho a}{\mu}(\operatorname{Ref}$ III-7);
2nd Regime - $\quad U_{b}=\sqrt{\frac{\sigma}{r_{b} \rho}+a r_{b}}(\operatorname{Ref}$ III-8);

where

$r_{b}=$ bubble radius,

$\mathrm{U}_{\mathrm{b}}=$ buoyant rise velocity.

For large bubbles that assume a spherical cap shape, the bubble radius is based on an equivalent spherical volume.

Acting against the buoyant rise of the bubble is the flow of the liquid out of the tank. By assuming that there is a uniform radial velocity distribution, as shown in Fig. III-47, the flow velocity can be expressed as a function of $r$, the distance from the outlet:

$\mathrm{U}_{\mathrm{f}}=\frac{\mathrm{Q}}{4 \mathrm{r}^{2} \cos ^{-1}\left(\frac{\mathrm{r}}{2 \mathrm{R}}\right)}$

where

$\mathrm{U}_{\mathrm{f}}=\mathrm{flow}$ velocity,

$r=$ distance to outlet,

$\mathrm{R}=$ tank radius,

$Q=$ volumetric flow rate.

The vector components of the two velocities, $U_{b}$ and $U_{f}$, were neglected (a conservative simplification), and the two velocities were set equal to determine the value of $\mathbf{r}$ at which the buoyant forces are equal to the momentum forces acting on the bubble. The equilibrium condition is plotted, with a solid line, for Flox in Fig. III-48. Above the line, bubbles will be buoyed away from the outlet; below the line, these bubbles will be drawn into the outlet. 


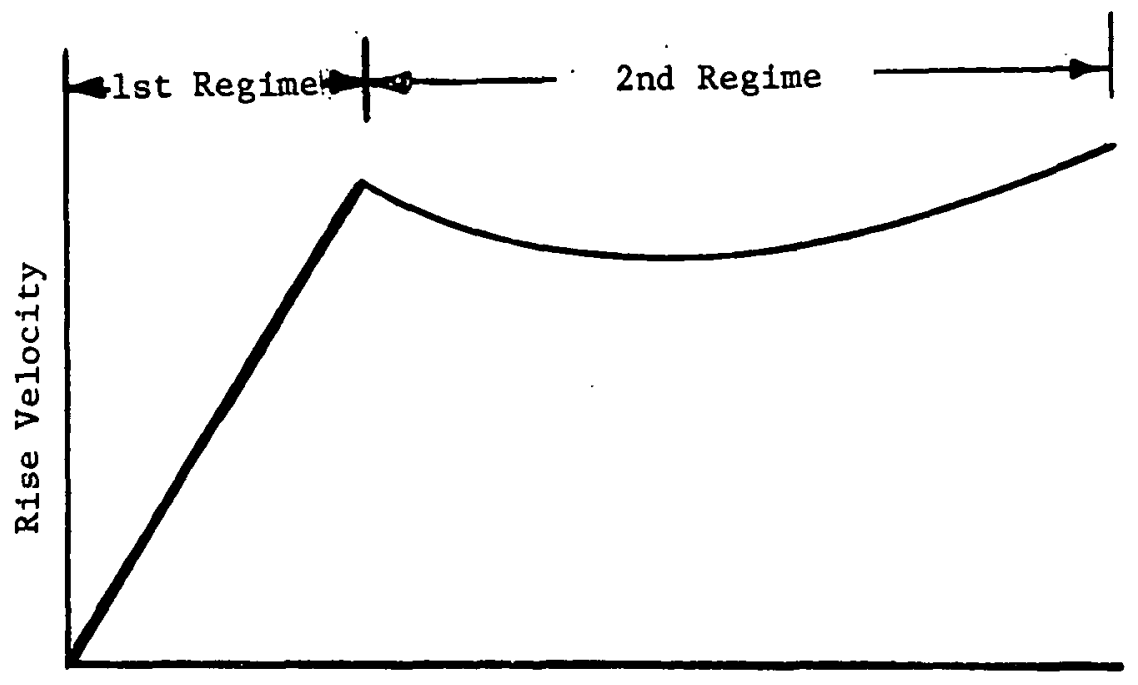

Bubble Radius

Fig. III-46 Rise Rate vs Bubble Radius for Low M Liquids

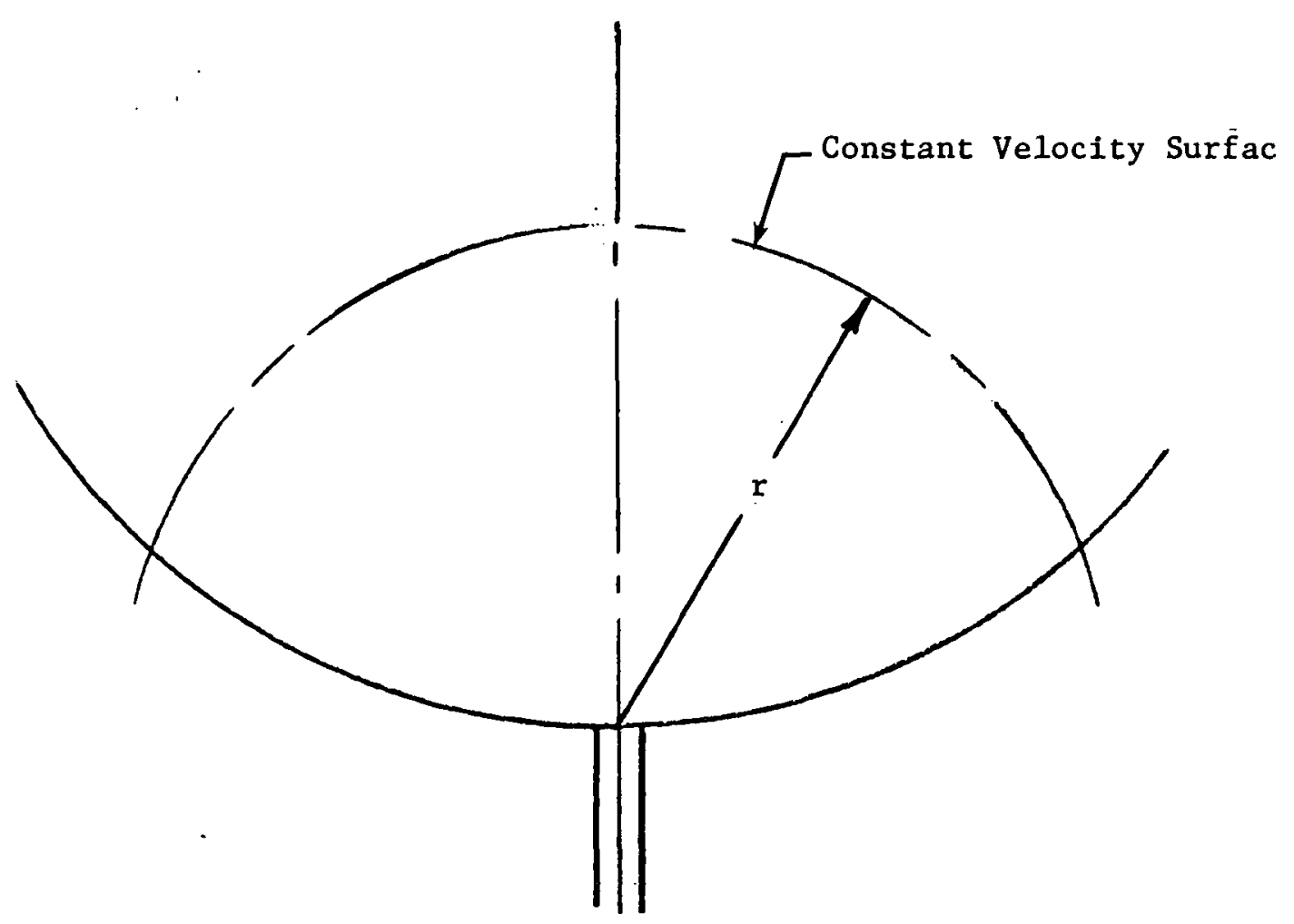

Fig. III-47 Flow Velocity Profile 


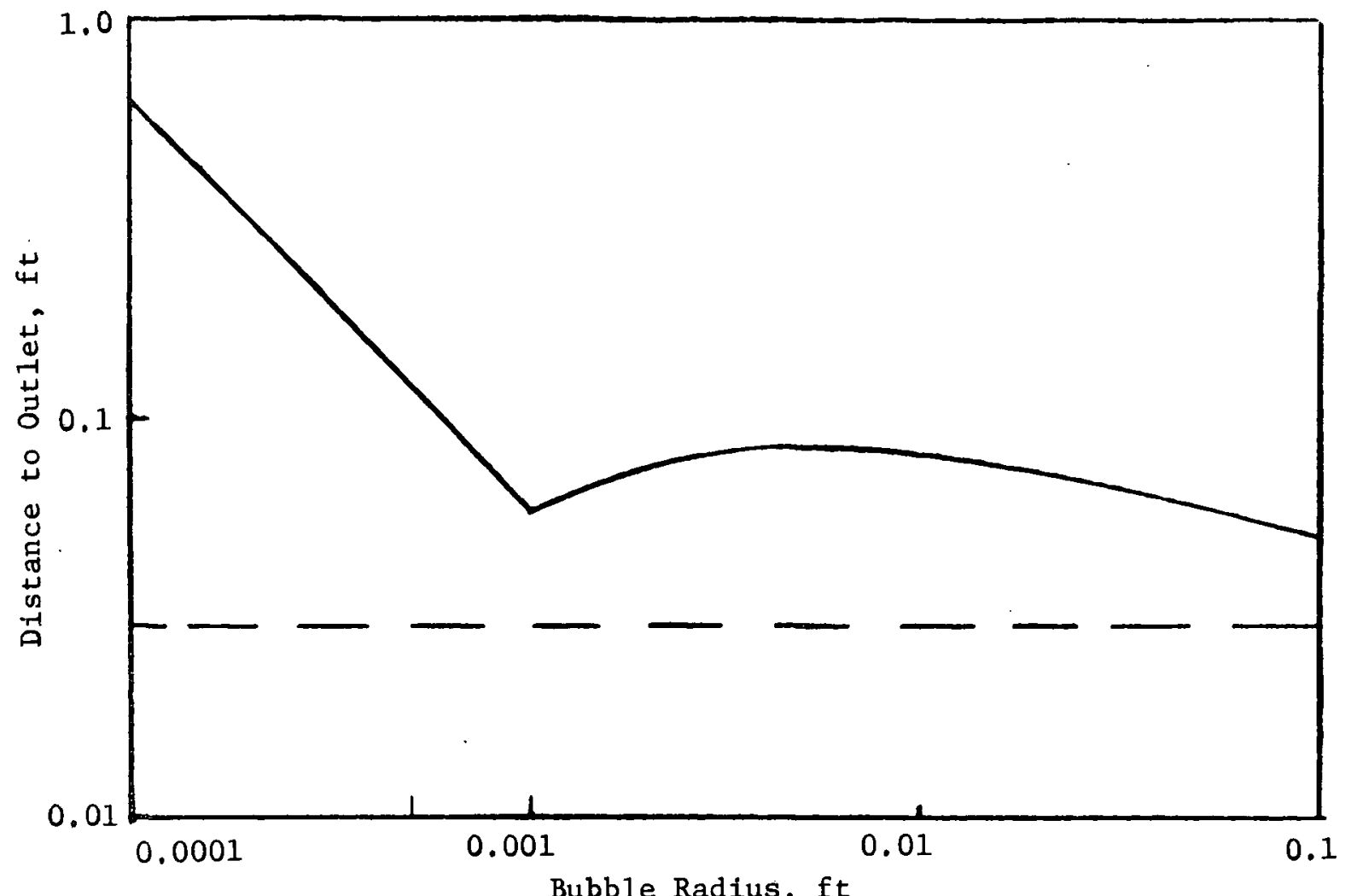

Fig. III-48 Bubble Equilibrium Conditions for Flox

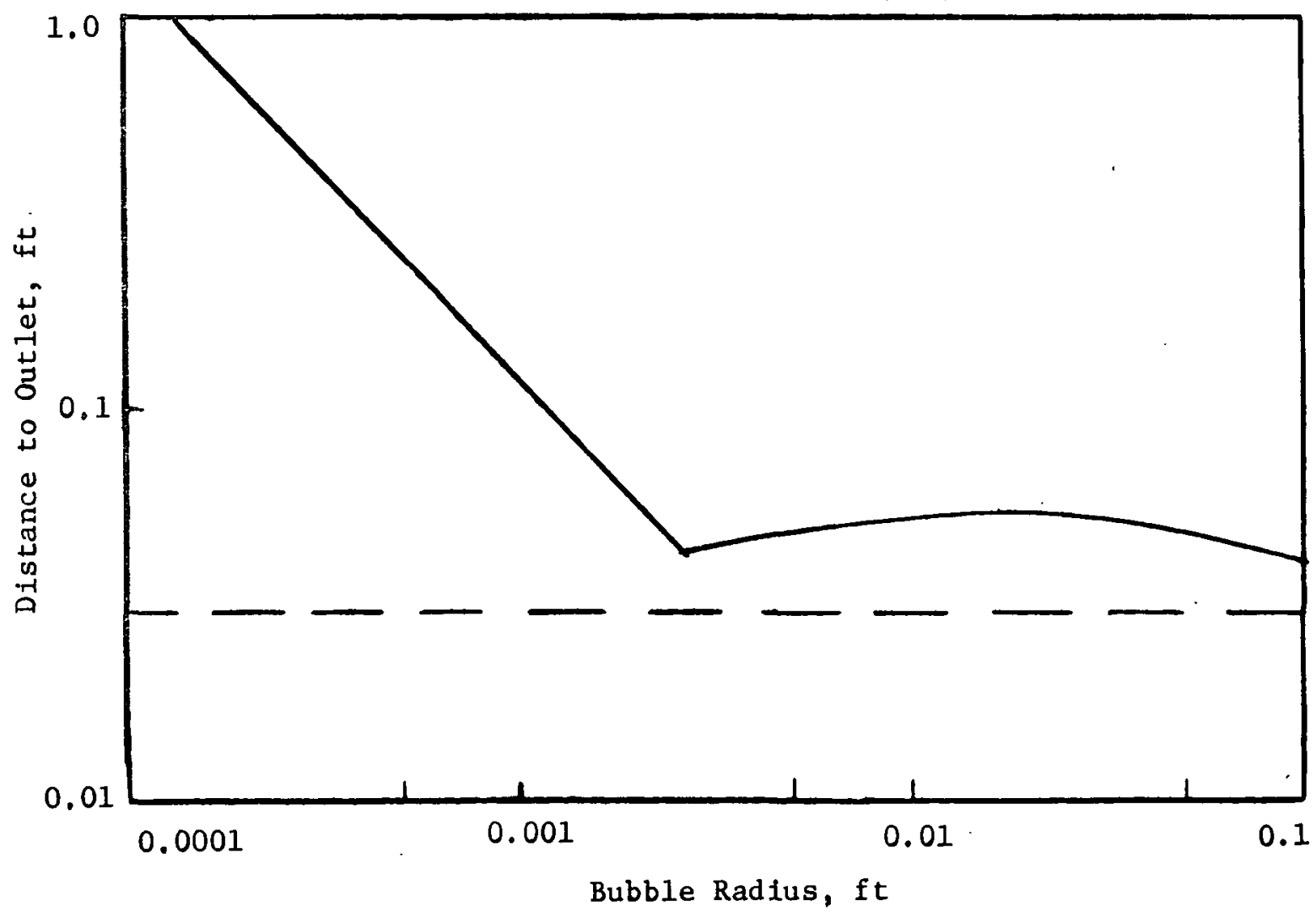

Fig. III-49 Bubble Equilibrium Conditions for MMH 
The dashed line in Fig. III-48 represents the radius at which the flow would enter a 0.75-in. diameter feed line and no further increase in flow velocity will occur. Only the simple outlet, as shown in Fig. III-47, is being considered at this point. The area between the two curves represents those bubble radii and distances to the tank outlet for which the bubble will be drawn toward the outlet. Repeating the analysis for the MMH tank produced the curves shown in Fig. III-49. Similar results would be obtained for the other propellant combinations.

Based on this analysis, it can be seen that the most critical factor is the distance between the outlet and the point at which the bubbles are formed. Only very small bubbles (less than 0.01 in. in diameter) would be ingested if no bubbles were formed within $2 \mathrm{in.}$ of the outlet. If a relatively stagnant volume of liquid can be held over the outlet, the problem of bubble ingestion is virtually eliminated. Minimizing the amount of splashing that occurs during settling will also reduce the effect of this problem. A device that will maintain a relatively flat interface in zero $g$, without any sizable amounts of liquid held up and away from the outlet, is the most effective approach for eliminating bubble ingestion.

An actual tank outlet would initially be much larger than the outlet evaluated. A tank outlet flange, typically 9 in. in diameter, is the only restriction on the size of the outlet. The outlet would make a transition to the 0.75-in.-diameter feedline further downstream. Consider the effect of an outlet 2 in. in diameter. The maximum flow velocity would be reduced sufficiently to eliminate the second regime of bubble sizes from consideration. The dashed lines in Fig. III-48 and III-49 would be moved upward so that the two curves intersect at a much smaller bubble radius. To conclude, proper design of the acquisition device and the tank outlet will eliminate the bubble ingestion problem.

d. Gas Ingestion Diring Outflow - At low liquid volumes, gas can be drawn into the tank outlet during outflow if the flowrate overwhelms the effect of surface tension and gravity (which act to maintain the flow of liquid). A dip will form in the interface that will rapidly accelerate toward the outlet and gas will then be ingested. The effects of this phenomenon can be approximated by using available information (Ref III-9 and III-10), but the effect of the device must be neglected. 
The Marker and Cel1 computer program (Ref III-11 and III-12) was used in the analysis so that most of the effects could be considered. Outflow from the Flox tank to perform the last burn was selected as the case to evaluate. The post device and the interface under zero-g conditions were input as the initial conditions (Fig. III-50). An axisymmetric cylindrical cell mesh closely approximates the small segment of the spherical tank within the boundaries of the problem. The dashed line on the figure shows where the spherical tank wall would be and is for reference only. At time zero, the liquid begins to flow out of the tank and a constant acceleration due to engine operation acts on the liquid. Surface tension was included in the calculations. These conditions were selected for the problem because they are both worst case as $f a r$ as the mission requirements are concerned and are representative of all the Category $B$ and $C$ acquisition devices.

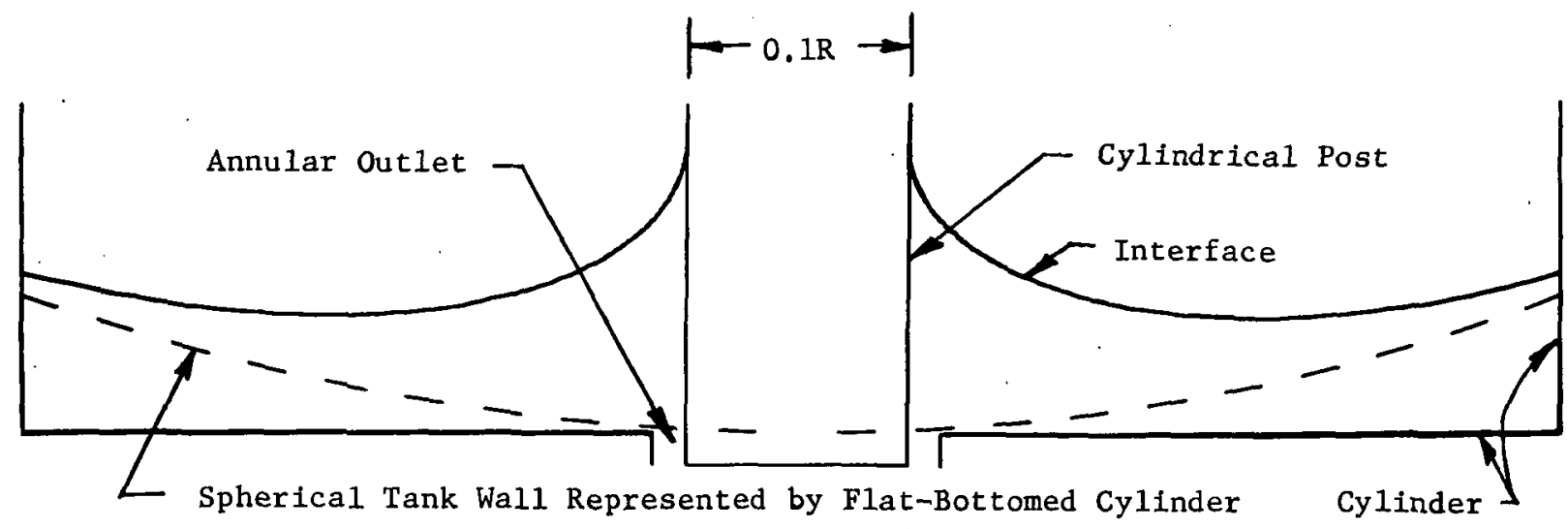

$0.001 \mathrm{Sec}$

Fig. III-50 Initial Conditions for Marker and Cell Computations

The calculated liquid motion is shown in Fig. III-5l. At first the liquid held about the post settles and the interface becomes flat. A dip begins to form after $0.15 \mathrm{sec}$ and by $0.28 \mathrm{sec}$ it has been drawn into the outlet. This burn has a duration of $3 \mathrm{sec}$ so it is obvious that suction dip is a problem for the post device with the worst-case outlet configuration analyzed. 


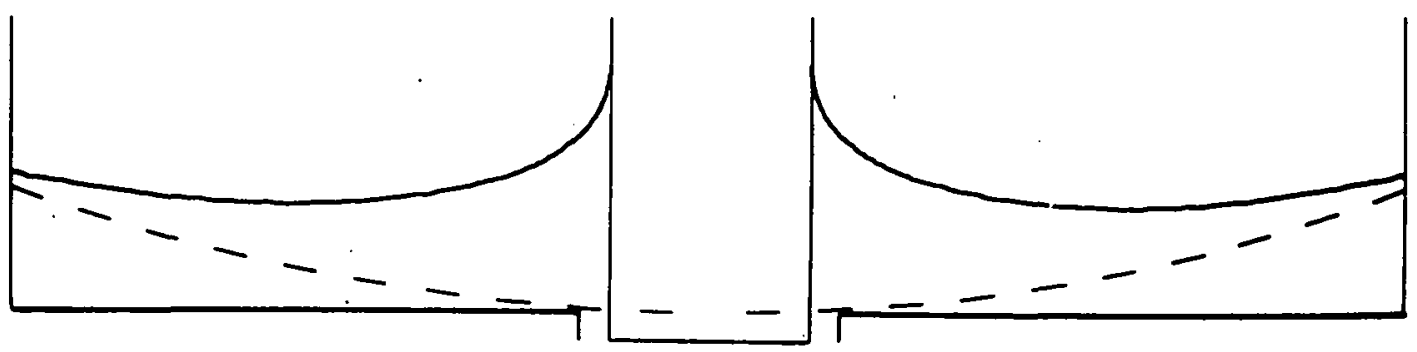

$0.050 \mathrm{sec}$

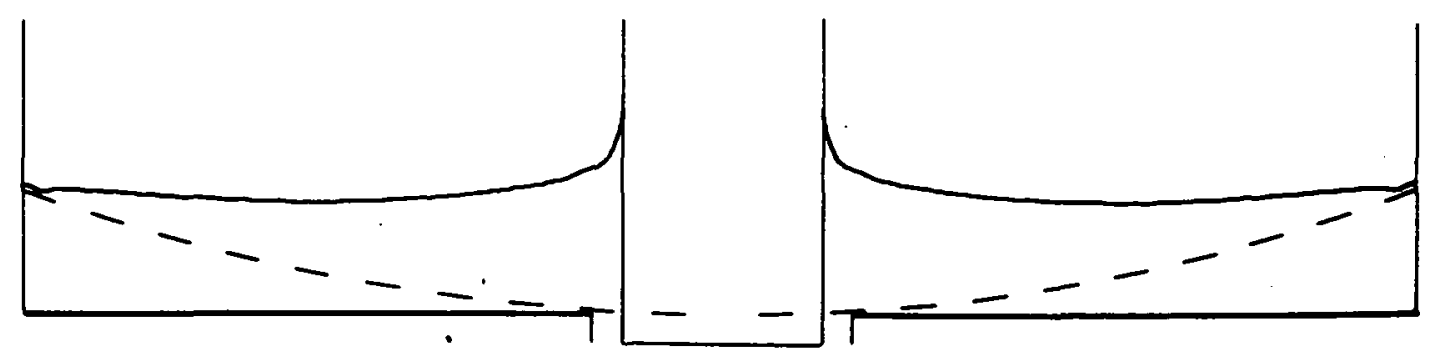

$0.100 \mathrm{sec}$

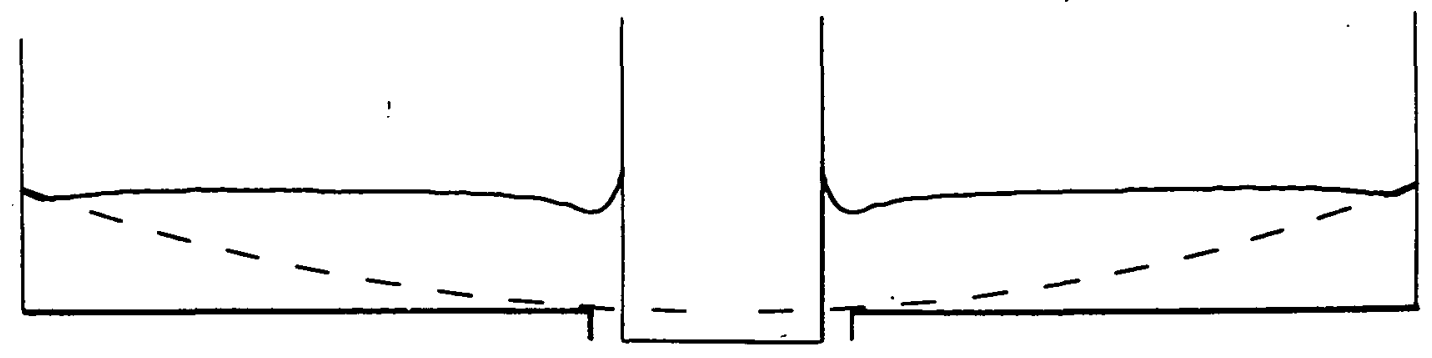

$0,150 \mathrm{sec}$

Fig. III-51 Marker and Cell Solution to Propelzont Draining Problem 


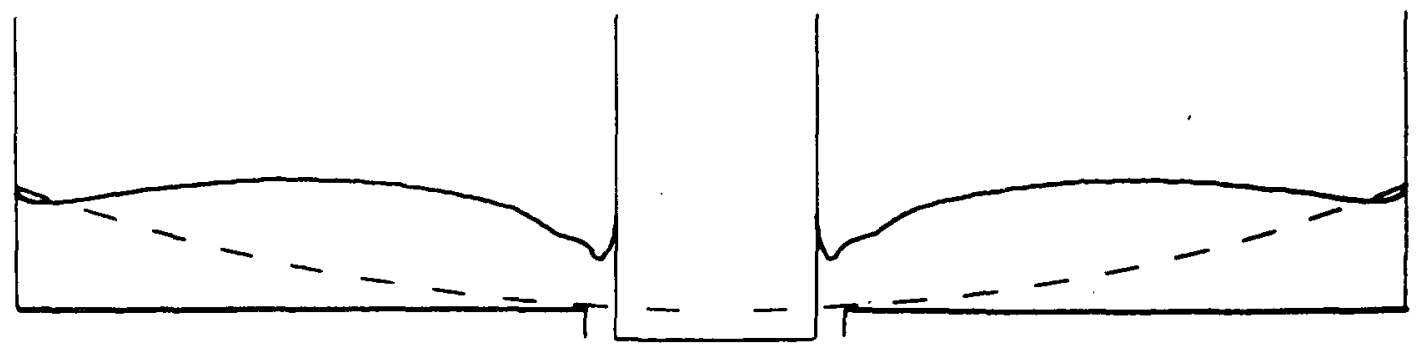

$0.200 \mathrm{sec}$

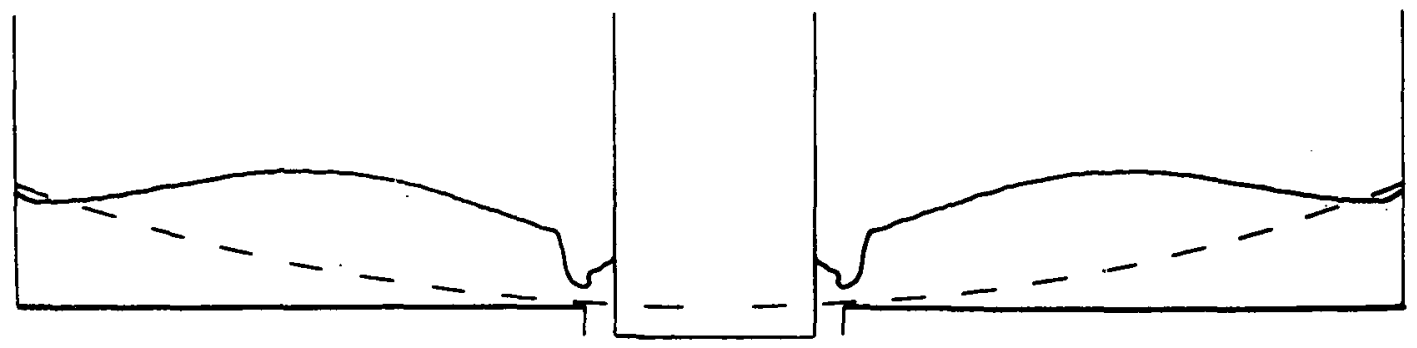

$0,250 \mathrm{sec}$

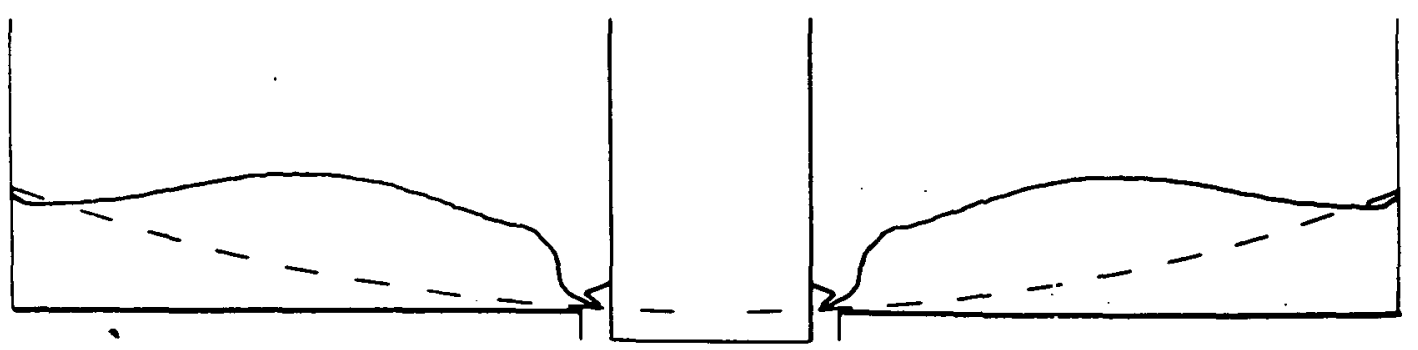

$0.280 \mathrm{sec}$

Fig. III-51 (concluded) 
Other outlet configurations were not analyzed with the computer model. Variations, which would improve the expulsion efficiency, include increasing the outlet area to decrease the flow velocity and contouring the outlet to provide a more uniform velocity profile. The other Category $B$ and $C$ devices will hold the liquid closer to the outlet, which would improve the expulsion efficiency. Experimental methods would be used to verify the ability of any of the devices (and outlet configuration) to expel liquid at very low volumes. 
An assessment of the interaction between the propellant acquisition subsystem and other spacecraft subsystems was made to support the analysis of candidate concepts. Spacecraft subsystems interacting with the propellant acquisition subsystem are the boom deployment system, the attitude control system (ACS), the engine gimbaling system, the pressurization system, the prope1lant tank thermal control system, and the ground hold cooling system. The propellant tank itself is considered to be a part of the acquisition system. Baseline tanks for this study were spherical; however, cylindrical tanks were also considered (see Chapter VI, Section C).

Interactions with the boom deployment system, the ACS, and the engine gimbal system were discussed previously in section A of this chapter. The dynamics associated with pressurant inflow and venting are considered in Chapter VI, Section B, and integration of a ground hold cooling coil, required for space storable propellants, is discussed in Section $C$ of this chapter. This leaves the pressurization system and the propellant tank thermal control system interactions, which are discussed in this section.

\section{Pressurization System Analysis}

Pressurization system operation and its effect on propellant acquisition system functioning was analyzed using two Martin Marietta computer programs. The 0D041 Propellant Tank Pressurization Program (Ref III-13) was used to determine propellant tank temperatures, pressures, and helium usage over the mission coast, pressurization, and burn sequence. A helium gas expansion program was used to calculate helium inlet temperatures for input to the OD041 program. The procedure followed in employing the computer programs was to first run the OD041 program assuming a constant inlet helium temperature. The resulting helium usage data were then input to the helium expansion program to obtain the helium inlet temperature profile during mission pressurization and burn periods. The helium inlet temperature profile was then input to the 0D041, keeping all other input the same. The change in helium usage requirements between the two 0D041 runs was negligible (approximately $1 \%$ for the oxidizer tank and $0.2 \%$ for the fuel tank). However, tank pressure levels varied appreciably because of the decreasing helium inlet temperature during burn periods, followed by the increasing ullage temperature during coast periods. 
One of the major assumptions in the analysis was that heat transfer between the propellant tank and the environment was zero (adiabatic process). However, the propellant tank and the pressurant storage tank were connected so that heat transfer could occur between tanks.

The initial conditions for pressures, temperatures, volumes, and propellant flow rates, as well as definitions of structural materials were taken from the propulsion system criteria presented in Chapter I. The Flox/MMH combination was employed. To simplify the analysis and minimize computer time, a modified burn profile consisting of 10 bum periods was established. Since the first five burn periods of the baseline mission consume approximately $89 \%$ of the propellant, these burn periods were not changed in the modified mission. Rather, the major change was to combine the 22 orbital trim burns into five burns while maintaining the total burn time constant. Since adiabatic conditions were assumed external to the propellant tank, calculations by the program during coast periods consisted of establishing equilibrium conditions within the tank between the propellant, ullage gas, and tank wall. As equilibrium conditions are established rather rapidly, the coast-period durations were also reduced in the computer runs to reduce computational time. Table III- 8 presents the modified trajectory correction propulsion event schedule employed in the analysis. The prepressurization periods prior to each burn were assumed to be 5 sec long.

The first information generated by the pressurization system analysis was the definition of system weight for nominal propellant temperature conditions. These data, presented in Table III-9, were used in vehicle $\mathrm{cg}$ calculations.

The next consideration evaluated in the pressurization system analysis was the maximum pressures obtained in nonvented propellant tanks. Nonvented propellant tanks are desirable from the standpoint of system compatibility and performance predictability. However, high tank pressures require thicker-walled (heavier) propellant tanks. Data from the computer runs indicate that, if no venting is permitted, significant pressure rise will occur in both propellant tanks during the coast periods after the third, fourth, and fifth burns. During these burns, $86 \%$ of the total propellant is expelled. Pressure histories for both the Flox and MMH tanks are presented in Fig. III-52. The pressure rises could affect the operation of the propellant acquisition system by producing the following transient effects: 
Table III-8 Modified Trajectory Correction Propulsion Event Schedule

\begin{tabular}{|c|c|c|c|c|}
\hline \multirow[b]{2}{*}{ Event } & \multicolumn{3}{|c|}{ Duration } & \multirow[b]{2}{*}{ Comments } \\
\hline & $\begin{array}{l}\text { Coast, } \\
\text { hr }\end{array}$ & $\begin{array}{l}\text { Prepressuri- } \\
\text { zation, sec }\end{array}$ & $\begin{array}{l}\text { Burn, } \\
\text { sec }\end{array}$ & \\
\hline $\begin{array}{l}\text { Midcourse } \\
\text { Correction }\end{array}$ & 0 & 5.0 & 10.1 & $\begin{array}{l}\text { No prepressurization } \\
\text { occurred since tanks } \\
\text { were assumed pres- } \\
\text { surized on the ground. }\end{array}$ \\
\hline $\begin{array}{l}\text { Pre-encounter } \\
\text { Maneuver } \\
\text { First Retro- } \\
\text { Maneuver at } \\
\text { Periapse }\end{array}$ & 500 & 5.0 & 11.1 & \\
\hline $\begin{array}{l}\text { Plane Change } \\
\text { at Apoapse } \\
\text { Final Retro- } \\
\text { Maneuver at } \\
\text { Periapse }\end{array}$ & 722 & 5.0 & 156.4 & \\
\hline Orbit Trims & & & & \\
\hline 1 & 360 & 5.0 & 23.5 & (Orbit Trims 1-7) \\
\hline 2 & 1000 & 5.0 & 16.5 & (Orbit Trim 8) \\
\hline 3 & 336 & 5.0 & 11.1 & (Orbit Trims 9-15) \\
\hline 4 & 1000 & 5.0 & 16.1 & (Orbit Trim 16) \\
\hline 5 & 900 & 5.0 & 19.0 & (Orbit Trims 17-22) \\
\hline Total & 6374 & 50.0 & 790.0 & \\
\hline
\end{tabular}

Table III-9 Pressurization System Requirements for Nominal Propelzant Temperature Conditions

\begin{tabular}{|c|c|c|c|c|}
\hline & Flox & MMH & $\mathrm{F}_{2}$ & $\mathrm{~N}_{2} \mathrm{H}_{4}$ \\
\hline Propellant Temperature, ${ }^{\circ} \mathrm{R}$ & 155 & 530 & 155 & 530 \\
\hline $\begin{array}{l}\text { Initial Helium Storage Pressure, } \\
\text { psia }\end{array}$ & 3444 & 3785 & 3444 & 3785 \\
\hline $\begin{array}{l}\text { Final Helium Storage Pressure, psia } \\
\text { Helium Storage Container }\end{array}$ & 600 & 600 & 600 & 600 \\
\hline Weight, ${ }^{1 b} \mathrm{~m}$ & 20.4 & 16.6 & 16.4 & 23.6 \\
\hline Volume, $\mathrm{ft}^{3}$ & 1.4 & 0.64 & 1.1 & 0.91 \\
\hline Helium Loaded, ${ }^{1 b}{ }_{m}$ & 8.27 & 1.48 & 6.45 & 2.1 \\
\hline
\end{tabular}



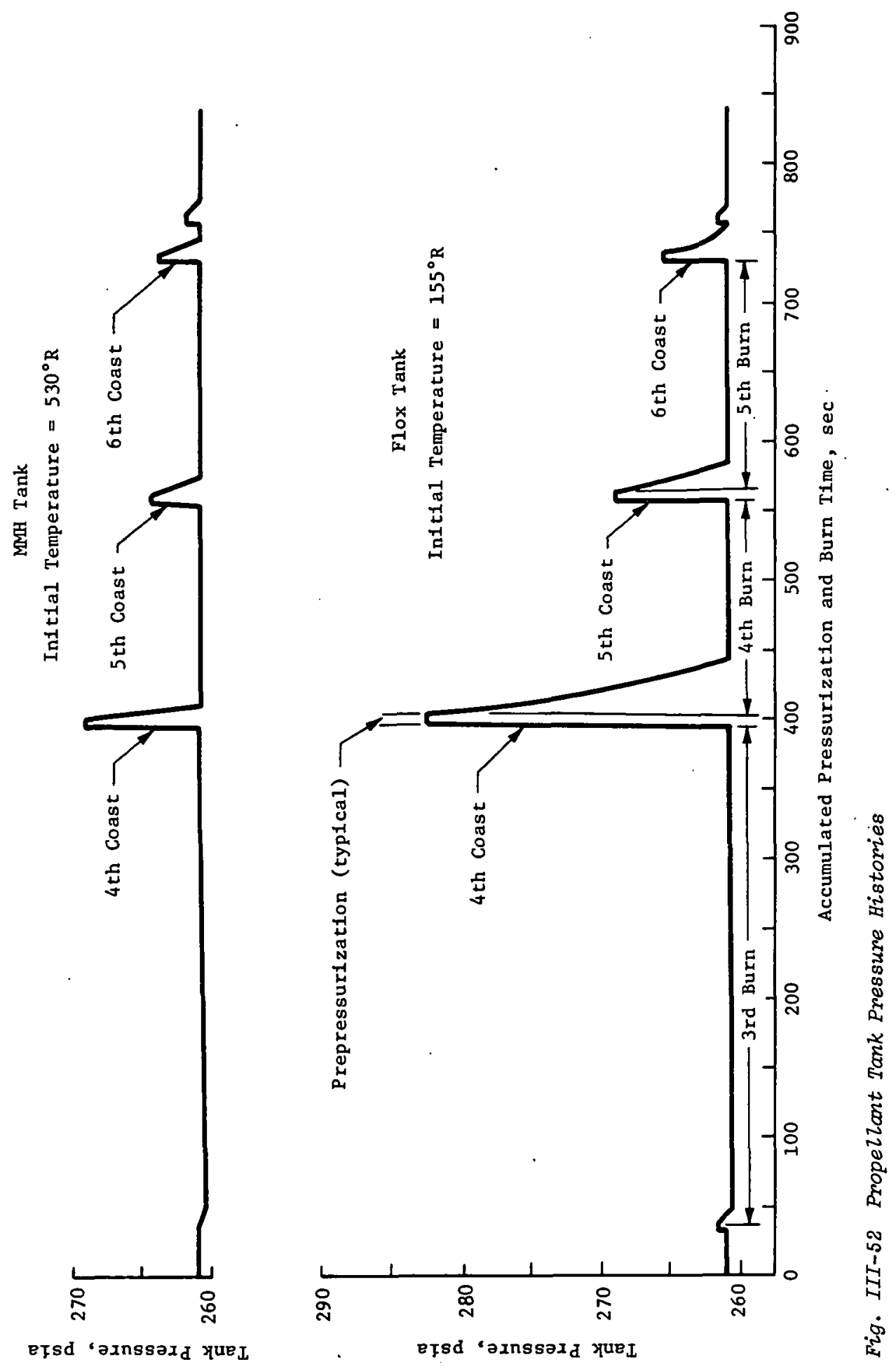
1) Initial propellant flow rates higher than design values;

2) Propellant mixture ratio shifts;

3) Thrust and acceleration variations from nominal; and

4) Premature propellant dropout.

An investigation into the causes of the overpressure conditions indicated that the pressure increases were mainly attributed to warming during coast periods of the ullage gas which was previously cooled by expansion from the storage container during outflow. As can be seen in Fig. III-52, the overpressure conditions in the Flox tank were more severe than those in the MMH tanks. The highest Flox tank pressure occurred in the coast period following the third burn when the pressure reached a value of 283 psia. In this analysis, it was assumed that the propellant tanks were prepressurized to operating pressure before launch. Because of the small ullage volumes involved, it was thought that a similar overpressure condition might also occur after the first and second burn periods if the tanks were initially prepressurized in flight rather than on the ground. A third factor also believed significant in contributing to the overpressure condition was the duration of the prepressurization period before a burn. These factors were evaluated using the 0D041 Tank Pressurization Program. The evaluation was performed on the Flox tank since the overpressure conditions were largest in this tank.

The initial tank pressure used in the computer program runs was a nominal blanket pressure of 18 psia instead of the normal regulated tank pressure. A nominal pressure of 240 psia and a prope1lant temperature of $150^{\circ} \mathrm{F}$ were assumed. Since the tank overpressure conditions in small ullages were of primary concern, the program was run for only the first four burn periods of the modified mission in Table III-8.

To evaluate the effect of the helium inlet temperature, two cases were considered. The first case considered the normal propulsion system arrangement shown by solid lines in Fig. III-53 and assumed that the helium was stored at the Flox temperature ( $150^{\circ} \mathrm{R}$ storage). During gas expansion and flow for prepressurization and burn periods, the helium temperature entering the tank decreased. The second inlet temperature case assumed that the helium pressurant was supplied at $530^{\circ} \mathrm{R}$ to the Flox tank from the MMH tank pressurization system storage tank. Helium transfer from the fuel side to the oxidizer side would be accomplisined by adding a valve and line, as shown by the dashed lines in Fig. III-53. To evaluate the effect of prepressurization duration, times of 60,180 , and $300 \mathrm{sec}$ were employed.

III-80 


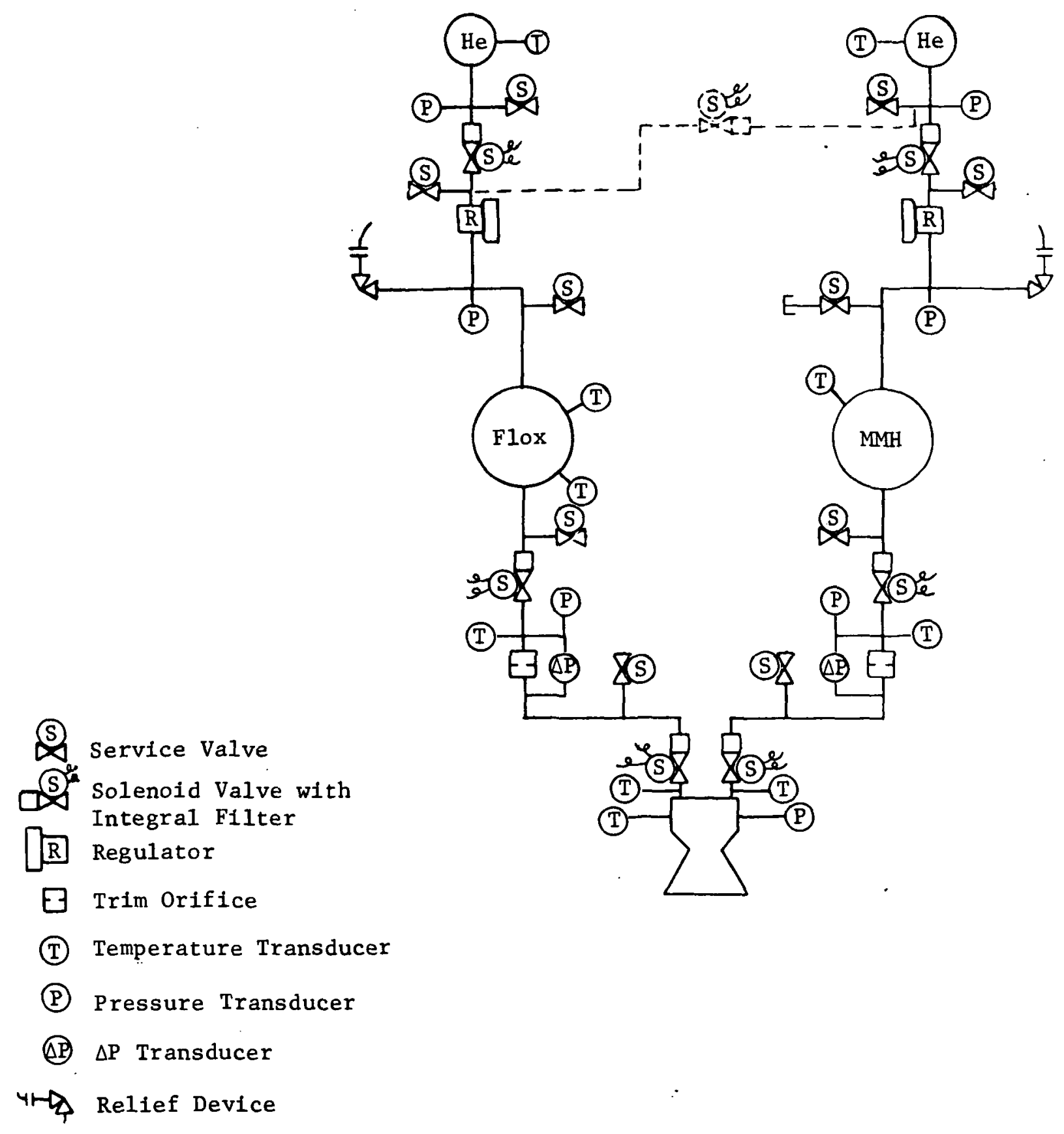

Fig. III-53 Flox/MMH Propulsion Subsystem 
The Flox tank pressure history for a 60-sec prepressurization is presented in Fig. III-54. During coast periods after both first and second burns, the tank pressures were found to decrease for both $150^{\circ} \mathrm{R}$ and $530^{\circ} \mathrm{R}$ helium storage conditions. Even though the helium inlet temperature for the $150^{\circ} \mathrm{R}$ storage case decreased because of the expansion process in the storage container, subsequent compression of the helium in the ullage during prepressurization raised the ullage gas temperature above the propellant temperature. Since the first two burn periods were very short, the warmer ullage gas did not cool to the propellant temperature before the end of the burn. During the coast period following each burn, however, sufficient time was available for the ullage gas to cool to the propellant temperature. This produced the observed pressure decreases. During the third prepressurization period with $150^{\circ} \mathrm{R}$ helium, compression heating of the ullage gas again occurred. However, the third burn period was extremely long compared to the first two burns so that more time was available for heat transfer between the ullage gas and propellant. In addition, the helium inlet temperature decreased from an initial value of $150^{\circ} \mathrm{R}$ to a final value of $105^{\circ} \mathrm{R}$ due to expansion in the storage container over the long burn period. This drop in inlet temperature reduced the ullage gas temperature at the end of the third burn to a value approximately $10^{\circ} \mathrm{R}$ less than the $150^{\circ} \mathrm{R}$ bulk propellant temperature. In reaching equilibrium during the third coast period, the ullage gas warmed to the propellant temperature and produced the pressure rise indicated in Fig. III-54.

For the $530^{\circ} \mathrm{R}$ storage case, the tank pressure decreased as expected during the coast periods after each burn. The decrease in helium inlet temperature due to expansion in the storage container was not considered; the helium inlet temperature was maintained constant at $530^{\circ} \mathrm{R}$. However, even if the temperature change due to the expansion process had been included, the helium inlet temperature would never drop below the Flox temperature and the ullage gas temperature at the end of the burn periods would always be above the propellant temperature. Therefore, the cooling process during the coast period would still reduce the tank pressure, al though not to the extent shown in Fig. III-54.

An evaluation of the data for $180-$ and 300-sec prepressurization durations indicated the same basic trends as the 60-sec case. The maximum tank pressures obtained during each coast period for the different cases considered are compared in Table III-10. In general, longer prepressurization periods resulted in higher pressures. The differences are relatively small, however, so that prepressurization duration does not appear to be a significant factor. The helium mass requirements for each prepressurization and burn period are shown in Table III-11. These data also indicate that prepressurization duration does not significantly influence the amount of helium required.

III -82 


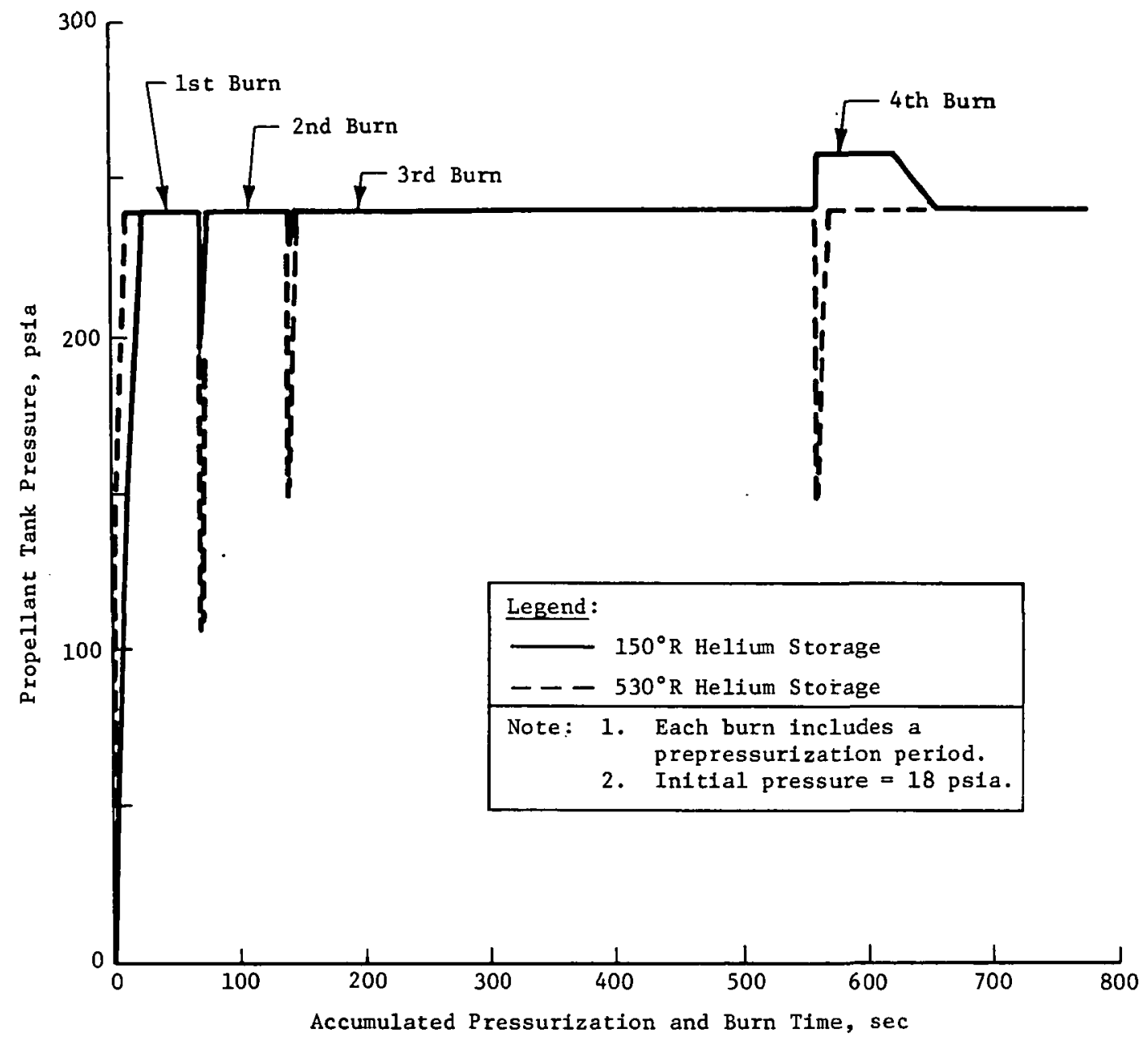

Fig. III-54 Propellant Tank Pressure History for 60-Second Prepressurization Duration 
Table III-10 Tank Pressures at the End of Coast

\begin{tabular}{|l|l|l|l|}
\hline $\begin{array}{l}\text { Prepressurization } \\
\text { Duration, sec }\end{array}$ & $\begin{array}{l}\text { Coast } \\
\text { Period }\end{array}$ & $\begin{array}{l}150^{\circ} \mathrm{R} \text { Storage, } \\
\text { psia }\end{array}$ & $\begin{array}{l}530^{\circ} \text { R Storage, } \\
\text { psia }\end{array}$ \\
\hline 60 & 1 & 196 & 107 \\
& 2 & 229 & 150 \\
& 3 & 259 & 147 \\
\hline 180 & 1 & 205 & 121 \\
& 2 & 233 & 167 \\
& 3 & 259 & 151 \\
\hline 300 & 1 & 209 & 127 \\
& 2 & 235 & 174 \\
& 3 & 259 & 153 \\
\hline
\end{tabular}

Table III-11 Helium Usage, $2 b_{m}$

\begin{tabular}{|l|l|l|l|l|l|l|}
\hline \multirow{2}{*}{$\begin{array}{l}\text { Prepressurization Duration } \\
\text { sec }\end{array}$} & \multicolumn{3}{|c|}{$150^{\circ} \mathrm{R}$ Storage } & \multicolumn{3}{c|}{$530^{\circ} \mathrm{R}$ Storage } \\
\cline { 2 - 7 } & 60 & 180 & 300 & 60 & 180 & 300 \\
\hline First Pressurization & 1.293 & 1.378 & 1.405 & 0.647 & 0.758 & 0.810 \\
First Burn & 0.111 & 0.098 & 0.097 & 0.040 & 0.035 & 0.026 \\
\hline Second Pressurization & 0.243 & 0.209 & 0.195 & 0.330 & 0.378 & 0.380 \\
Second Burn & 0.090 & 0.087 & 0.088 & 0.040 & 0.016 & 0.030 \\
\hline Third Pressurization & 0.063 & 0.042 & 0.033 & 0.231 & 0.220 & 0.220 \\
Third Burn & 3.077 & 3.067 & 3.062 & 1.213 & 1.169 & 1.149 \\
\hline Fourth Pressurization & -- & -- & -- & 0.467 & 0.531 & 0.552 \\
Fourth Burn & 1.005 & 0.997 & 1.011 & 0.535 & 0.468 & 0.455 \\
\hline \multicolumn{1}{|c|}{ Total } & 5.882 & 5.878 & 5.891 & 3.502 & 3.575 & 3.622 \\
\hline
\end{tabular}


Characteristics of the envisioned thermal control system for the spacecraft can also significantly affect the propellant tank pressure during long coast periods in the mission. The maximum variation in propellant temperature that could result from tolerances in the thermal control system operation is $\pm 17^{\circ} \mathrm{R}$. To estimate the impact of this temperature variation. on tank pressure, the equilibrium temperature obtained from the 0D041 computer runs for each coast period was increased by $17^{\circ} \mathrm{R}$. Assuming the same helium and propellant masses, a new ullage volume was calculated for the increased temperature. Using the same helium mass with the new ullage volume and increased temperature, a new helium partial pressure was calculated, assuming ideal gas relations. The helium partial pressure was then added to the propellant vapor pressure corresponding to the increased propellant temperature to get the new propellant tank pressure. Results for the 60-sec prepressurization duration runs are presented in Table III-12. The $150^{\circ} \mathrm{R}$ storage cases produce higher pressure rises resulting from the $17^{\circ} \mathrm{R}$ temperature increase than the $530^{\circ} \mathrm{R}$ storage cases because of the larger helium mass contained in the ullage.

Several conclusions have been reached from the results of this analysis. If nominal propellant temperatures are maintained, prepressurization of small ullages in the Flox tank before short burn periods does not require the use of high temperature inlet helium to prevent overpressure conditions from occurring. The tank pressure history in Fig. III-54 illustrates this condition.

Table III-12 Propelzant Tonk Temperature and Pressure Before and After $17^{\circ} \mathrm{R}$ Increase in Equilibrium Temperature $160-$ sec Pressurization)

\begin{tabular}{|c|c|c|c|c|c|}
\hline & \multicolumn{2}{|c|}{$150^{\circ} \mathrm{R}$ Storage } & \multicolumn{2}{|c|}{$530^{\circ} \mathrm{R}$ Storage } \\
\hline & & Before & After & Before & After \\
\hline $\begin{array}{l}\text { lst } \\
\text { Coast }\end{array}$ & $\begin{array}{l}\text { Temperature, }{ }^{\circ} \mathrm{R} \\
\text { Tank Pressure, psia }\end{array}$ & $\begin{array}{l}150 \\
196\end{array}$ & $\begin{array}{l}167 \\
270\end{array}$ & $\begin{array}{l}151 \\
107\end{array}$ & $\begin{array}{l}168 \\
156\end{array}$ \\
\hline $\begin{array}{l}\text { 2nd } \\
\text { Coast }\end{array}$ & $\begin{array}{l}\text { Temperature, }{ }^{\circ} \mathrm{R} \\
\text { Tank Pressure, psia }\end{array}$ & $\begin{array}{l}150 \\
229\end{array}$ & $\begin{array}{l}167 \\
310\end{array}$ & $\begin{array}{l}152 \\
150\end{array}$ & $\begin{array}{l}169 \\
213\end{array}$ \\
\hline $\begin{array}{l}3 \text { rd } \\
\text { Coast }\end{array}$ & $\begin{array}{l}\text { Temperature, }{ }^{\circ} \mathrm{R} \\
\text { Tank Pressure, psia }\end{array}$ & $\begin{array}{l}150 \\
259\end{array}$ & $\begin{array}{l}167 \\
317\end{array}$ & $\begin{array}{l}154 \\
147\end{array}$ & $\begin{array}{l}171 \\
189\end{array}$ \\
\hline
\end{tabular}


However, if a propellant temperature variation of $17^{\circ} \mathrm{R}$ is superimposed on the results in Fig. III-54, severe tank pressure conditions can develop when $150^{\circ} \mathrm{R}$ helium is used. Table III-12 compares tank pressures during coast for both $150^{\circ} \mathrm{R}$ and $530^{\circ} \mathrm{R}$ storage temperatures and a $17^{\circ} \mathrm{R}$ propellant temperature change. The resulting tank pressures for the $150^{\circ} \mathrm{R}$ cases are all above the nominal 240 psia tank pressure. These overpressure conditions would impact on the propellant acquisition system operation by creating transient variations in engine propellant flow rates, propellant mixture ratios, and thrust. Also premature outage could result. Therefore, use of $530^{\circ} \mathrm{R}$ helium or some other approach may be required.

In general, use of $530^{\circ} \mathrm{R}$ helium inlet temperature appears preferable for both short- and long-burn periods. However, one very important factor not considered is the aspect of total system weight. From previous analytical work performed by Martin Marietta in support of Contract NAS7-754 (Ref III-14), it was found that pressurization system weight savings of approximately $25 \%$ can be obtained if low temperature helium storage is employed. With low temperature storage, however, the propellant tank overpressure conditions would require heavier tanks. The overpressure conditions could be circumvented or at least minimized by negating the helium cooling due to expansion from the storage container. This could be accomplished by routing the helium from the $150^{\circ} \mathrm{R}$ storage tank through a feedline heat exchanger before introduction into the propellant tank. The system would be designed to maintain the helium temperature at the heat exchanger outlet equal to or very near the propellant feed temperature (approximately $150^{\circ} \mathrm{R}$ ). Additional analysis is required to further evaluate the effect of $150^{\circ} \mathrm{R}$ versus $530^{\circ} \mathrm{R}$ helium storage on total propulsion sytem weight. Results of such a comparison would be pertinent to selection of the pressure storage conditions. A blowdown system should also be evaluated in the assessment to determine the lightest pressurization system.

The effect of pressurization system operation on propellant temperature was also a factor in the analysis. Propellant temperature influences the propellant surface tension, which controls reorientation time and liquid-gas interface shapes. It was found from the analysis that the total Flox temperature change from the beginning to the end of the mission was only $3^{\circ} \mathrm{R}$, while the temperature change for the MMH was less than $1^{\circ} \mathrm{R}$. The change in surface tension resulting from these temperature changes is approximately $3 \%$ for the $\mathrm{Flox}$ and $0.2 \%$ for the $\mathrm{MMH}$. These variations are not significant for any of the acquisition system designs under consideration. 
All of the work performed and the results obtained have been based upon the Flox propellant system. The results, however, should also be applicable to the fluorine tank of the $\mathrm{F}_{2} / \mathrm{N}_{2} \mathrm{H}_{4}$ propulsion system. Similar result.s would be expected with an $O F_{2}$ tank.

2. Propellant Tank Thermal Control Analysis

The impact of the thermal environment on the operation of the propellant acquisition device was evaluated. The Flox tank was selected for analysis because heat leak into the cryogenic propellant tank presents the worst heat transfer case for the propellant acquisition system. Two different heat leak conditions were considered. These were normal heat leaks through the propellant tank supports and feedline and the anomalous situation in which the tank was exposed to direct solar radiation by the loss of $1 \mathrm{sq} f t$ of insulation.

a. Normal Heat Leaks - The first step in this analysis was to estimate heat leaks into the propellant tank. For the support heat leaks, it was assumed that the Flox tank (spherical) was supported on the equator at four equally spaced points. From the reference configuration for the Jupiter Orbiter Spacecraft (JPL Drawing 10041593), an estimate of the tank support structure was developed as shown in Fig. III-55. Four structural members, identified as $1,2,3$, and 4 lead to each tank support point. It was assumed that supports 1 and 2 were identical, as were supports 3 and 4.

The estimated length of supports 1 and 2 was $32.6 \mathrm{in.}$, while that of supports 3 and 4 was 22.5 in. Three temperature levels in the support structure, as indicated in Fig. III-55, were defined as follows:

$\mathrm{T}_{\mathrm{F}}=$ fuel tank support temperature;

$\mathrm{T}_{\mathrm{O}}=$ oxidizer tank support temperature;

$\mathrm{T}_{\mathrm{E}}=$ engine support temperature.

The total heat flow into the tank support point is given as:

$Q_{S}=Q_{1}+Q_{2}+Q_{3}+Q_{4}$

where $Q_{1}, Q_{2}, Q_{3}$, and $Q_{4}$ are heat flow rates in structural members $1,2,3$, and 4 , respectively. Assuming steady state conditions and applying Fourier's 1aw: 


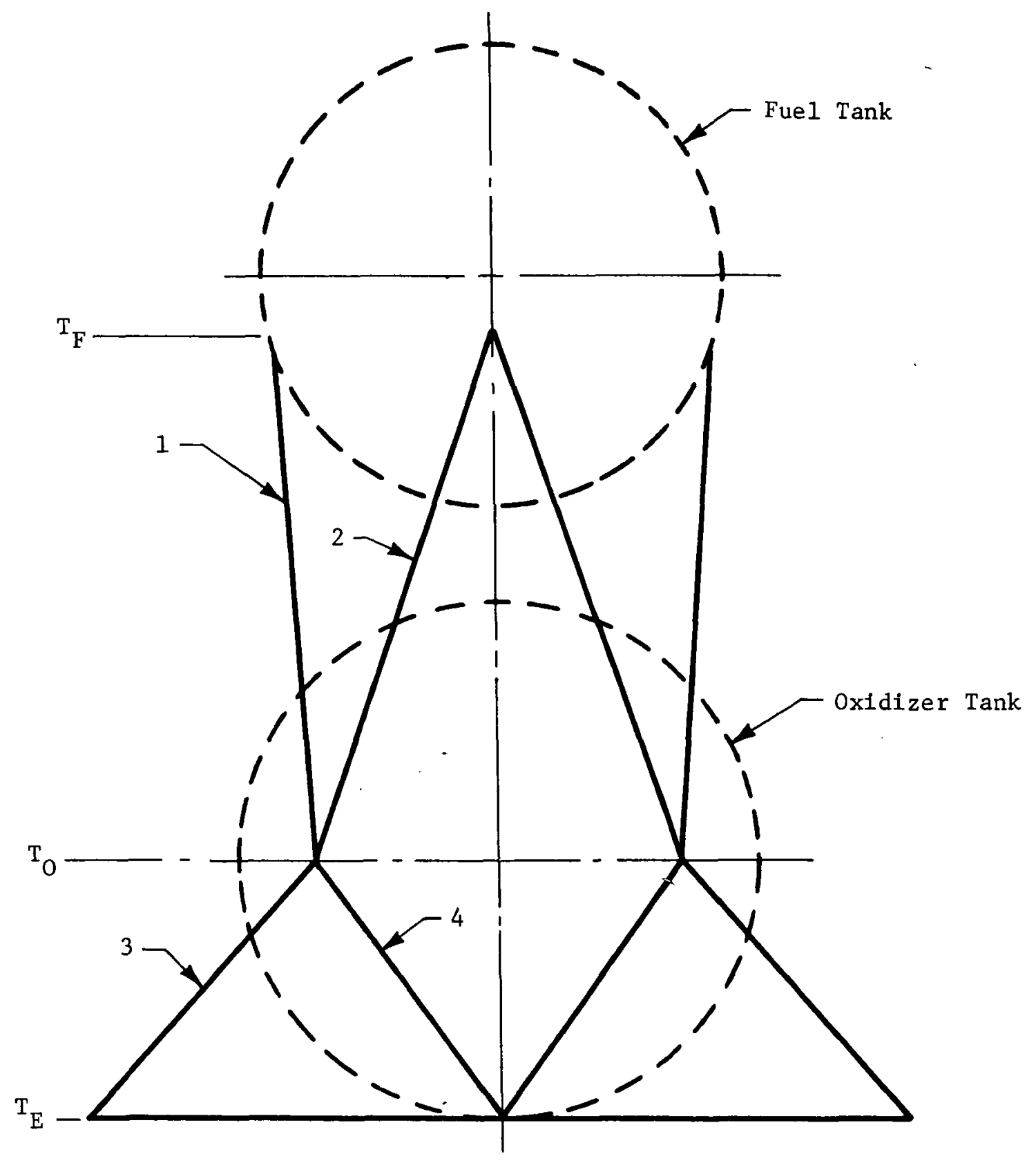

Fig. III-55 Flox Tank Support Structure 


$$
Q_{S}=\left[\frac{\mathrm{K}_{1} A_{1}}{L_{1}}+\frac{K_{2} A_{2}}{L_{2}}\right]\left(T_{F}-T_{0}\right)+\left[\frac{K_{3} A_{3}}{L_{3}}+\frac{K_{4} A_{4}}{L_{4}}\right]\left(T_{E}-T_{0}\right)
$$

where $\mathrm{K}=$ support thermal conductivity;

$$
\begin{aligned}
& A=\text { support cross section; } \\
& L=\text { support length. }
\end{aligned}
$$

Assuming the same material is used in all supports, the heat leak equation may be reduced to:

$Q_{S}=2 K A\left[\frac{T_{F}-T_{0}}{L_{1}}+\frac{T_{E}-T_{0}}{L_{3}}\right]$.

The structural material for support members was assumed to be tubular resin-impregnated fiberglass. Thermal conductivity and wall thickness of this material was obtained from Reference III15 as $0.15 \mathrm{Btu} / \mathrm{hr}-\mathrm{ft}-{ }^{\circ} \mathrm{R}$ and $0.030 \mathrm{in.}$, respectively. Temperature assumptions for $\mathrm{T}_{F}, \mathrm{~T}_{O}$, and $\mathrm{T}_{E}$ were based on information obtained from Reference III-12 and computer data supplied by JPL. With $\mathrm{T}_{\mathrm{F}}=520^{\circ} \mathrm{R}, \mathrm{T}_{\mathrm{O}}=155^{\circ} \mathrm{R}$, and $\mathrm{T}_{\mathrm{E}}=176^{\circ} \mathrm{R}$, the heat leak into each tank support point was calculated to be $0.043 \mathrm{Btu} / \mathrm{hr}$. For the analysis, this value was increased approximately 10 times to a value of $0.5 \mathrm{Btu} / \mathrm{hr}$ for conservatism.

The second tank heat leak considered was that from the propellant feed line. This heat leak, $Q_{F L}$, was calculated by Fourier's 1 aw as :

$Q_{F L}=\frac{K_{L} A_{L}}{L_{L}}\left(T_{E}-T_{O}\right)$

where

$K_{L}=$ thermal conductivity of line;

$A_{L}=$ cross section of line material;

$L_{L}=$ length of line;

$\mathrm{T}_{\mathrm{E}}=$ engine temperature;

$\mathrm{T}_{0}=$ oxidizer tank temperature. 
The feed line was assumed to be 1-ft long and constructed of 2219 aluminum tube, 3/4-in. 0.D. with a 0.030-in. wall. A temperature of $155^{\circ} \mathrm{R}$ was assumed for the oxidizer tank while a value of $179^{\circ} \mathrm{R}$ was assumed for the engine based on data from Reference III-15 and the data. supplied by JPL. The resulting feed line heat leak was $0.436 \mathrm{Btu} / \mathrm{hr}$.

Next, a mathematical model was developed to calculate tank wall temperature and heat flux distributions resulting from the heat leaks. To simplify the analysis, it was assumed that the spirerical surface in the vicinity of each heat leak could be approximated by a circular fin, as illustrated in Fig. III-56. The heat leak, $Q$, enters the tank wall, as illustrated, and is distributed over the surface defined by the wall thickness, $\ell$, and an arbitrary radius, $r_{0}$. The lower surface of the fin is insulated as the tank external surface. The upper surface of the fin is covered by the propellant. Heat transfer from the fin into the propellant is governed by conduction in the propellant. An energy balance on the fin leads to the following differential equation:

$-\frac{1}{r} \frac{d}{d r}\left(r q_{r}\right)-\frac{q_{c}}{l}=0$

where

$r$ = fin radius;

$\mathrm{q}_{\mathrm{r}}=$ heat transfer in the fin in the $\mathrm{r}$ direction;

$\mathrm{q}_{\mathrm{c}}=$ heat transfer away from the fin into the propeliant;

$\ell=$ fin (tank wall) thickness.

Expressions for $q_{r}$ and $q_{c}$ are obtained from Fourier's 1 aw as follows:

$q_{r}=-K_{w} \frac{d T_{w}}{d r}$

$q_{c}=-k_{p} \frac{\left(T_{p}-T_{w}\right)}{L_{p}}$

[III-33] 


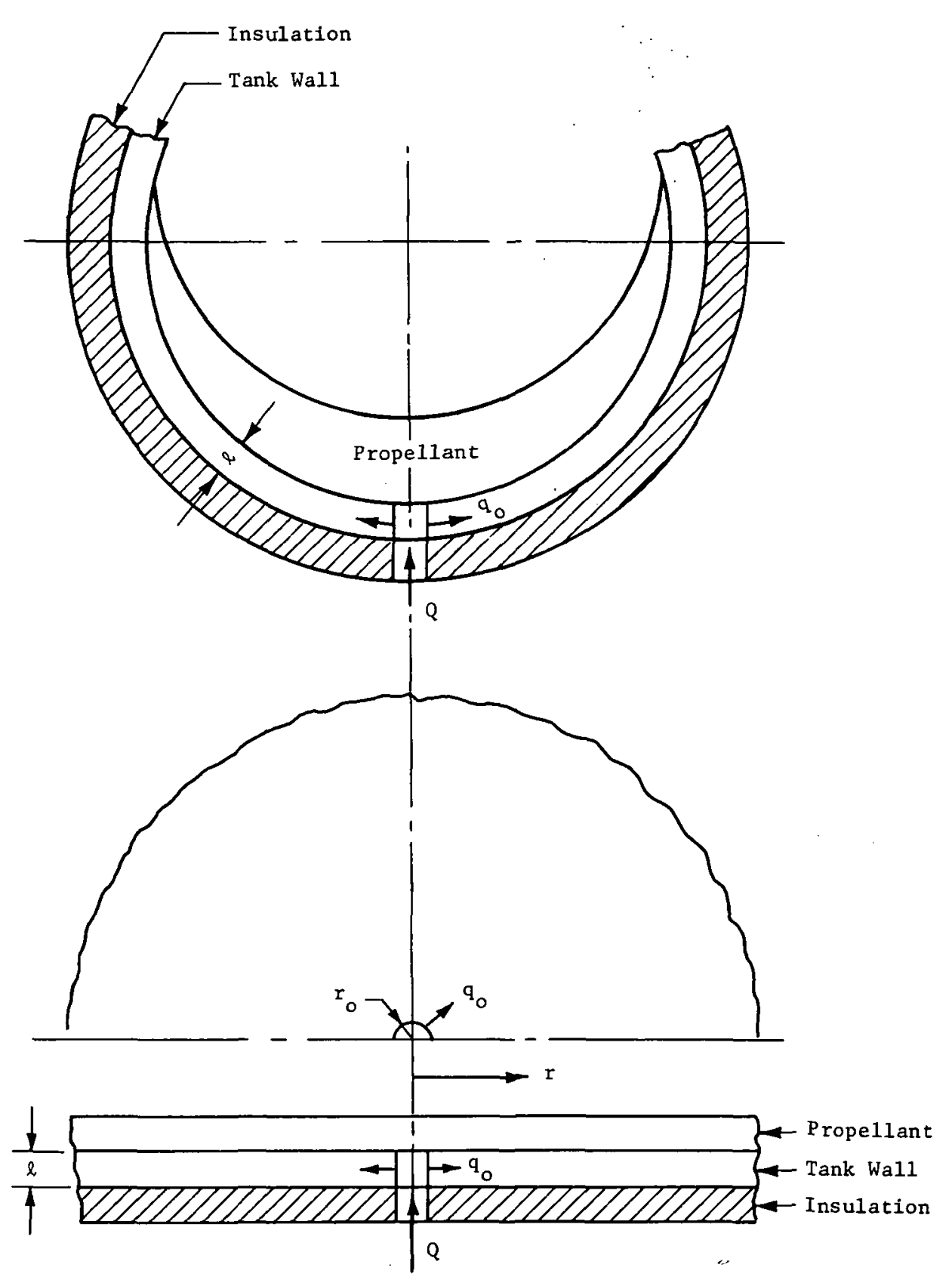

Fig. III-56 Circular Fin Simulation of Spherical Propellant Tank 
where

$\mathrm{T}_{\mathrm{w}}=\mathrm{wall}$ (fin) temperature;

$\mathrm{T}_{\mathrm{p}}=$ prope 11 ant temperature;

$\mathrm{K}_{\mathrm{w}}=$ wall thermal conductivity;

$\mathrm{K}_{\mathrm{p}}=$ propellant thermal conductivity;

$\mathrm{L}_{\mathrm{p}}=\begin{aligned} & \text { length of fluid over which temperature difference } \\ & \text { occurs. }\end{aligned}$

Substitution in the differential equation yields:

$\frac{\mathrm{d}^{2} \theta}{\mathrm{d} \mathrm{r}^{2}}+\frac{1}{\mathrm{r}} \frac{\mathrm{d} \theta}{\mathrm{dr}}-\frac{\mathrm{K}_{\mathrm{p}}}{\mathrm{K}_{\mathrm{W}}} \frac{\theta}{\ell \mathrm{L}_{\mathrm{P}}}=0$

where $\theta$ is defined as $T_{w}-T_{p}$.

This equation may be modified into the following Bessel's equation:

$r^{2} \frac{d^{2} \theta}{d r^{2}}+r \frac{d \theta}{d r}-m r^{2} \theta=0$

where $\mathrm{m}=\frac{\mathrm{K}}{\mathrm{K}} \mathrm{w}_{\mathrm{w}} \mathrm{L}_{\mathrm{p}}$.

A solution for this equation is obtained from Reference III-16 as follows:

$\theta=C_{1} I_{0}(\sqrt{m} r)+C_{2} K_{0}(\sqrt{m} r)$

where $I_{0}(\sqrt{\mathrm{m}} r)$ and $K_{0}(\sqrt{\mathrm{m}} r)$ are modified Bessel's functions of the first and second kind of order zero and $C_{1}$ and $C_{2}$ are arbitrary constants. The boundary conditions for the equation are:

1) At $r=r_{0}, \frac{d \theta}{d r}=-\frac{q_{o}}{K_{w}}=-\frac{Q}{2 \pi r_{o} \ell K_{w}}$;

2) At $r=\infty, \frac{\mathrm{d} \theta}{\mathrm{dr}}=0$.

III -92 
Applying the boundary conditions, leads to the following solution:

$\theta=T_{w}-T_{p}=\left(\frac{A}{\sqrt{m}}\right) \frac{K_{o}(\sqrt{m} r)}{K_{l}(\sqrt{m} r)}$

where

$A=\frac{Q}{2 \operatorname{lr}_{\mathrm{O}} \mathrm{K}_{\mathrm{W}} \ell}$

$\mathrm{m}=\frac{\mathrm{K}_{\mathrm{p}}}{\mathrm{K}_{\mathrm{w}} \mathrm{LL}_{\mathrm{p}}}$

This equation was employed to calculate the temperature distribution in the wall in the vicinity of both the tank support points and the outlet. For the tank supports, the following data were used:

$Q=0.5 \mathrm{Btu} / \mathrm{hr}$;

$r_{0}=1.0$ in.;

$\mathrm{K}_{\mathrm{W}}=36 \mathrm{Btu} / \mathrm{hr}-\mathrm{ft}-{ }^{\circ} \mathrm{R}$ (2219 aluminum);

$\ell=0.070$ in.;

$\mathrm{K}_{\mathrm{p}}=0.084 \mathrm{Btu} / \mathrm{hr}-\mathrm{ft}-{ }^{\circ} \mathrm{R}$.

For the propellant width, $\mathrm{L}_{\mathrm{p}}$, over which the temperature difference $\theta$ occurs, and arbitrary value of $1.0 \mathrm{in}$. was assumed. The resulting temperature difference, $T_{w}-T_{p}$, is presented in Fig. III-57 as a function of fin radius, $r$. For the feed line heat leak, the following data were used:

$\mathrm{Q}=0.436 \mathrm{Btu} / \mathrm{hr}$;

$r_{0}=0.375$ in.;

$\mathrm{K}_{\mathrm{W}}=36 \mathrm{Btu} / \mathrm{hr}-\mathrm{ft}-{ }^{\circ} \mathrm{R}$;

$\ell=0.070$ in.;

$\mathrm{K}_{\mathrm{p}}=0.084 \mathrm{Btu} / \mathrm{hr}-\mathrm{ft}-{ }^{\circ} \mathrm{R}$;

$\mathrm{L}_{\mathrm{p}}=1.0 \mathrm{in}$. 


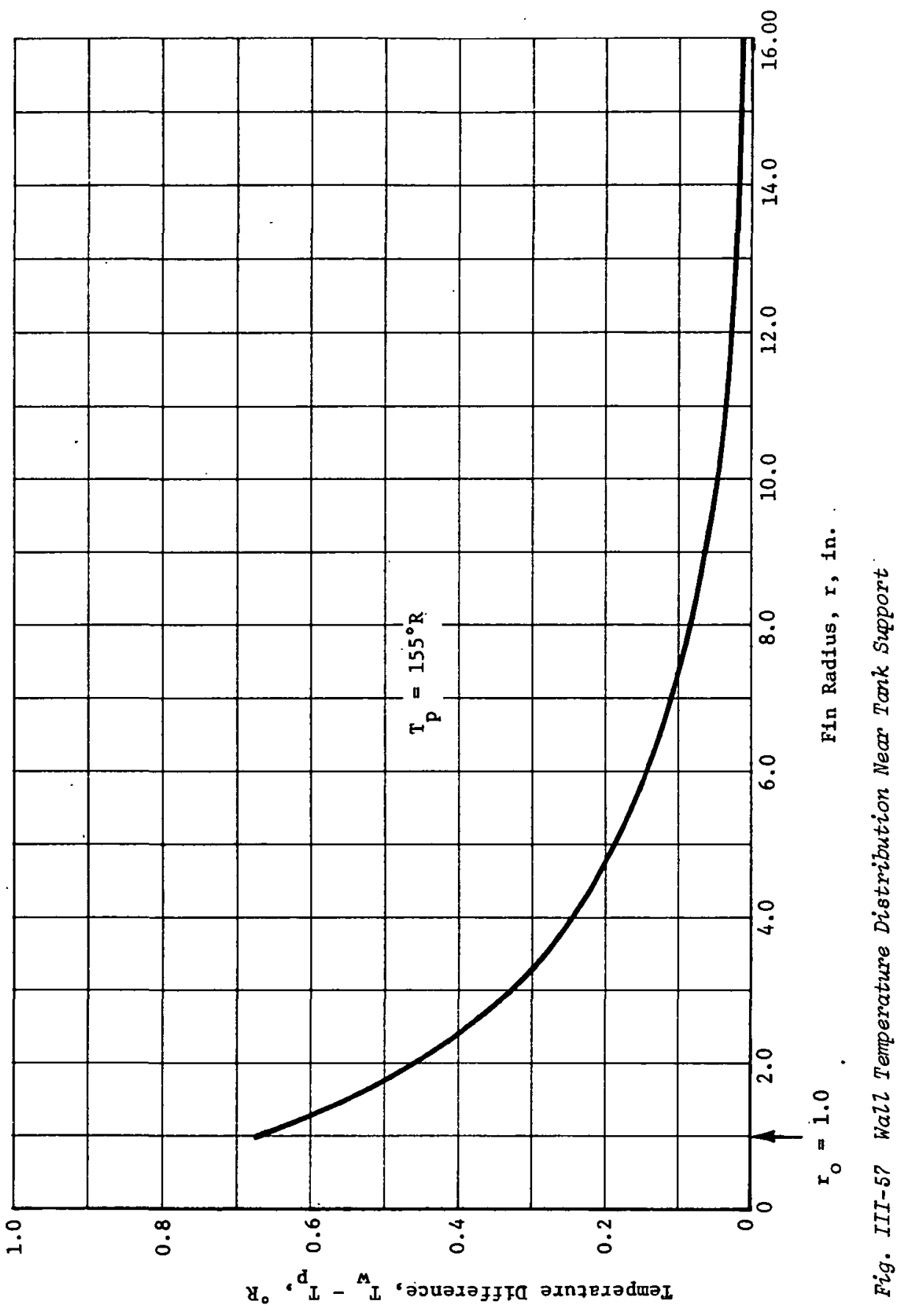


The calculated temperature difference is presented in Fig. III58. The maximum temperature difference between the tank wall and the propellant for either the tank support or feed line heat leak is less than $1^{\circ} \mathrm{R}$. Furthermore, the temperature differences decrease to essentially zero in relatively short distances. Thus, it appears that the normal tank heat leaks will not change propellant temperature and, therefore, surface tension significantly enough to affect performance of the propellant acquisition device. These results should also be applicable to the fluorine tank in the $\mathrm{F}_{2} / \mathrm{N}_{2} \mathrm{H}_{4}$ system and to both tanks in an $\mathrm{OF}_{2} / \mathrm{B}_{2} \mathrm{H}_{6}$ system.

b. Loss of Insulation - Loss of $1 \mathrm{sq} f t$ of foam insulation from the oxidizer tank was evaluated as part of the propellant tank thermal control analysis. Operation in this degraded condition was considered for both near-Earth and near-Jupiter environments. First, a steady-state analysis was performed. It was assumed that the surface was oriented normal to the sun and that the propellant tank wall in the vicinity of the lost insulation could be represented by a circular fin model, as shown in Fig. III-59. Two regions were defined for development of the analytical mode1. Region I is that area where the insulation was lost (bare tank wall), while Region II includes the adjacent tank wall still covered with insulation. An energy balance was constructed for both wall regions based on the following assumptions:

1) Steady state;

2) Thermal symetry about centerline;

3) Thermal gradient across the wall is zero;

4) Heat transferred into propellant is by conduction only;

5) Bulk temperature of propellant is constant; and

6) Exposed tank wall radiates to absolute zero.

From the energy balance, the following differential equation was developed for Region I:

$\frac{\mathrm{d}^{2} \mathrm{~T}_{W I}}{d r^{2}}+\frac{1}{r t} \frac{\mathrm{dT}_{W I}}{\mathrm{dr}}+\frac{\mathrm{q}_{i}}{\mathrm{~K}_{W} \mathrm{t}}-\frac{\mathrm{q}_{c}}{\mathrm{~K}_{W} \mathrm{t}}=0$ 


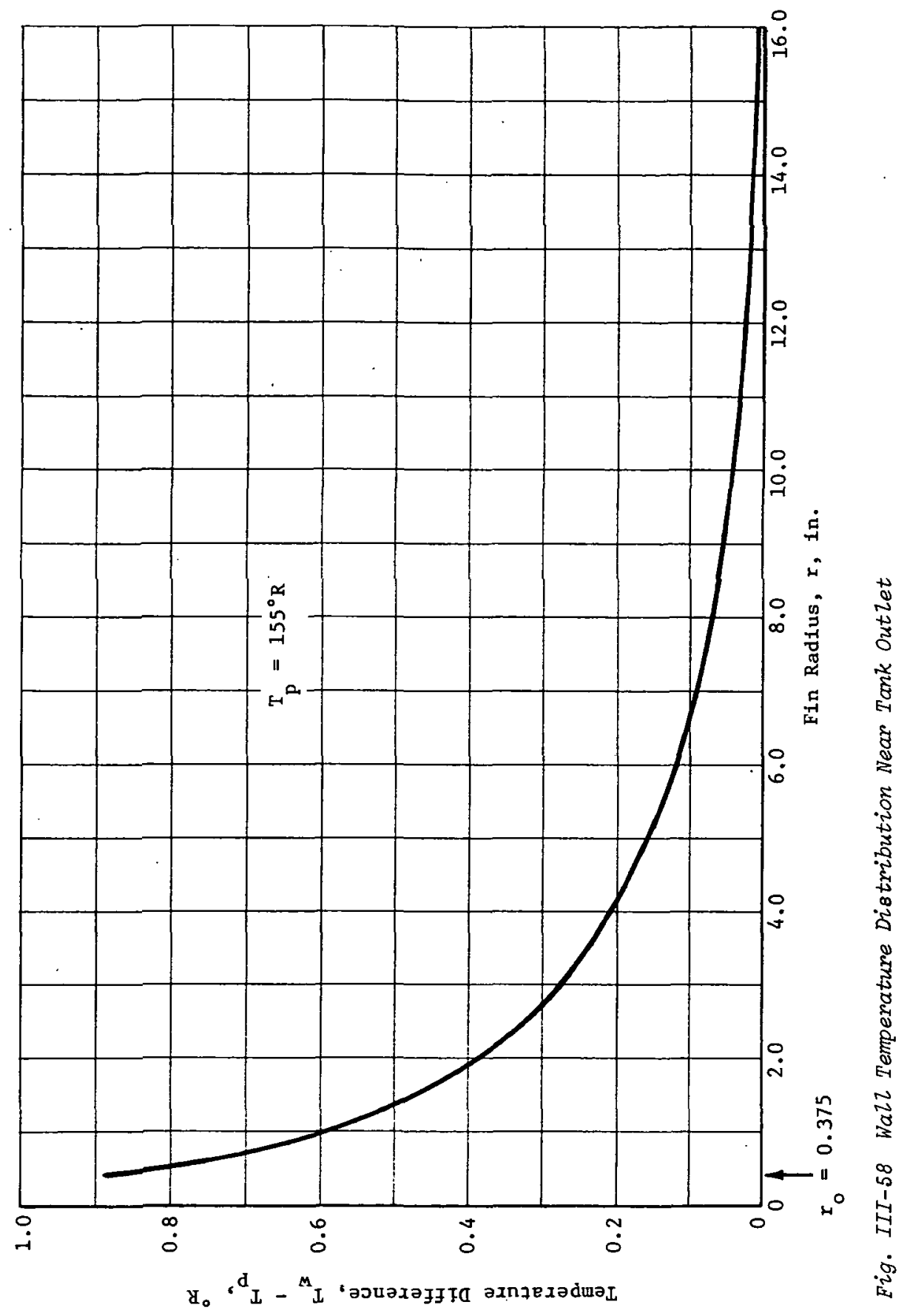




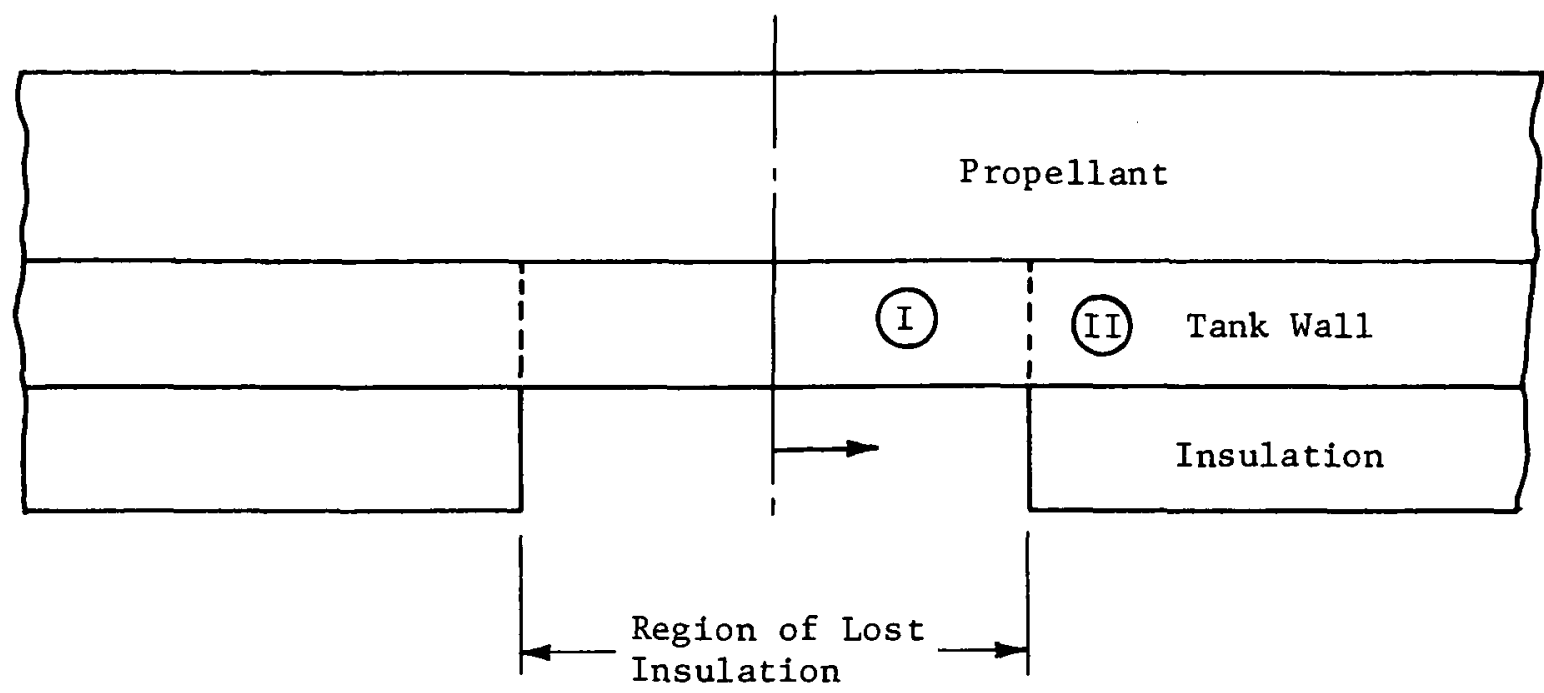

Fig. III-59 Circular Fin Simulation of Propellant Tank

where

$\mathrm{T}_{\mathrm{WI}}=$ wall temperature in Region $\mathrm{I}$;

$\mathbf{r}=$ fin (wall) radius;

$q_{i}=$ net heat entering Region I from solar radiation;

$q_{c}=$ heat conducted away from wall away into propellant;

$\mathrm{K}_{\mathrm{W}}=$ wall thermal conductivity;

t $=$ wall thickness.

The term $q_{i}$ is defined as follows:

$q_{i}=\alpha q_{s}-h_{r} T_{W I}$

where

$q_{S}=$ incident solar flux;

$\alpha$ = surface absorptivity;

$h_{R}=$ effective radiation heat transfer coefficient. 
The term $q_{c}$ is defined by Fourier's Law as:

$q_{c}=\frac{K_{p}}{L_{p}}\left(T_{W I}-T_{p}\right)$

whe re

$\mathrm{K}_{\mathrm{p}}=$ propellant thermal conductivity;

$\mathrm{L}_{\mathrm{p}}=$ thickness of propellant over which $\mathrm{T}_{\mathrm{WI}}-\mathrm{T}_{\mathrm{p}}$ occurs;

$\mathrm{T}_{\mathrm{P}}=$ propellant temperature.

From the energy balance for Region II, the following differential equation was developed:

$\frac{\mathrm{d}^{2} \mathrm{~T}_{W I I}}{d r^{2}}+\frac{1}{r} \frac{\mathrm{dT}_{W I I}}{\mathrm{dr}}-\frac{\mathrm{q}_{\mathrm{c}}}{\mathrm{K}_{W}{ }^{\mathrm{t}}}=0$

or

$\frac{\mathrm{d}^{2} \mathrm{~T}_{W I I}}{\mathrm{dr}^{2}}+\frac{1}{\mathrm{r}} \frac{\mathrm{dT}_{W I I}}{\mathrm{dr}}-\frac{\mathrm{K}_{\mathrm{p}}}{\mathrm{K}_{W} \mathrm{~L}_{\mathrm{p}} \mathrm{t}}\left(\mathrm{T}_{W I I}=\mathrm{T}_{\mathrm{p}}\right)=0$

where $\mathrm{T}_{\text {WII }}=$ wall temperature in Region II.

Solution of the differential equations from the energy balance yielded the following equations for wall temperature in Regions I and II, respectively:

$$
\begin{aligned}
& T_{W I}=\frac{B}{A}-\left[\frac{B C-A D}{A C}\right]\left[\frac{\sqrt{C} K_{1}\left(\sqrt{C} r_{1}\right) I_{0}(\sqrt{A} r)}{\sqrt{A} I_{1}\left(\sqrt{A} r_{1}\right) K_{0}\left(\sqrt{C} r_{1}\right)+\sqrt{C} I_{0}\left(\sqrt{A} r_{1}\right) K_{1}\left(\sqrt{C} r_{1}\right)}\right] \\
& T_{W I I}=\frac{D}{C}+\left[\frac{B C-A C}{A C}\right]\left[\frac{\sqrt{A} I_{1}\left(\sqrt{A} r_{1}\right) K_{0}(\sqrt{C} r)}{\sqrt{A} I_{1}\left(\sqrt{A} r_{1}\right) K_{0}\left(\sqrt{C} R_{1}\right)+\sqrt{C} I_{0}\left(\sqrt{A} r_{1}\right) K_{1}\left(\sqrt{C} r_{1}\right)}\right],
\end{aligned}
$$


where

$$
\begin{aligned}
& A=\left(\frac{h_{R}}{K_{W}}+\frac{K_{p}}{K_{W}^{L}}\right)\left(\frac{1}{t}\right), \\
& B=\left(\frac{K_{p}^{T} p}{K_{W} L_{p}}+\frac{\alpha q_{s}}{K_{W}}\right)\left(\frac{1}{t}\right), \\
& C=\frac{K_{p}}{K_{W}^{L} t}, \text { and } \\
& D=\frac{K_{p} T p}{K_{W}^{L} t} .
\end{aligned}
$$

Wall temperature profiles were computed for both near-Earth and near-Jupiter operation based on the following data:

\begin{tabular}{|l|l|l|}
\cline { 2 - 3 } \multicolumn{1}{c|}{} & Near Earth & Near Jupiter \\
\hline $\mathrm{q}_{\mathrm{S}}, \mathrm{Btu} / \mathrm{hr} \mathrm{ft}^{2} *$ & 430 & 16 \\
$\alpha$ & 0.9 & 0.9 \\
$\mathrm{~h}_{\mathrm{R}}, \mathrm{Btu} / \mathrm{hr} \mathrm{ft}^{3}{ }^{\circ} \mathrm{F}$ & 0.02 & 0.006 \\
\hline$*$ & & \\
\hline Solar heat flux data obtained from Ref III-15. \\
\hline
\end{tabular}

The resulting profiles are presented in Fig. III-60. These wal1 temperature profiles are applicable to either the Flox tank in the Flox/MMH propulsion system or the fluorine tank in the $\mathrm{F}_{2} / \mathrm{N}_{2} \mathrm{H}_{4}$ propulsion system. The wall temperature profile for the nearEarth orbit is higher than the approximately $260^{\circ} \mathrm{F}$ critical temperature of Flox. Therefore, this temperature would not actually be obtained since tank failure would occur before reaching these conditions. Thus, if $1 \mathrm{sq}$ ft of insulation is lost near-Earth and the area remains exposed to the sun, a catastrophic failure could result. Near Jupiter, the wall temperature profile is much less severe. However, the mass of propellant remaining is also much less so that the assumption of a constant bulk temperature may not be valid. In either the near-Earth or near-Jupiter cases, no consideration was given to possible effects of the spacecraft 


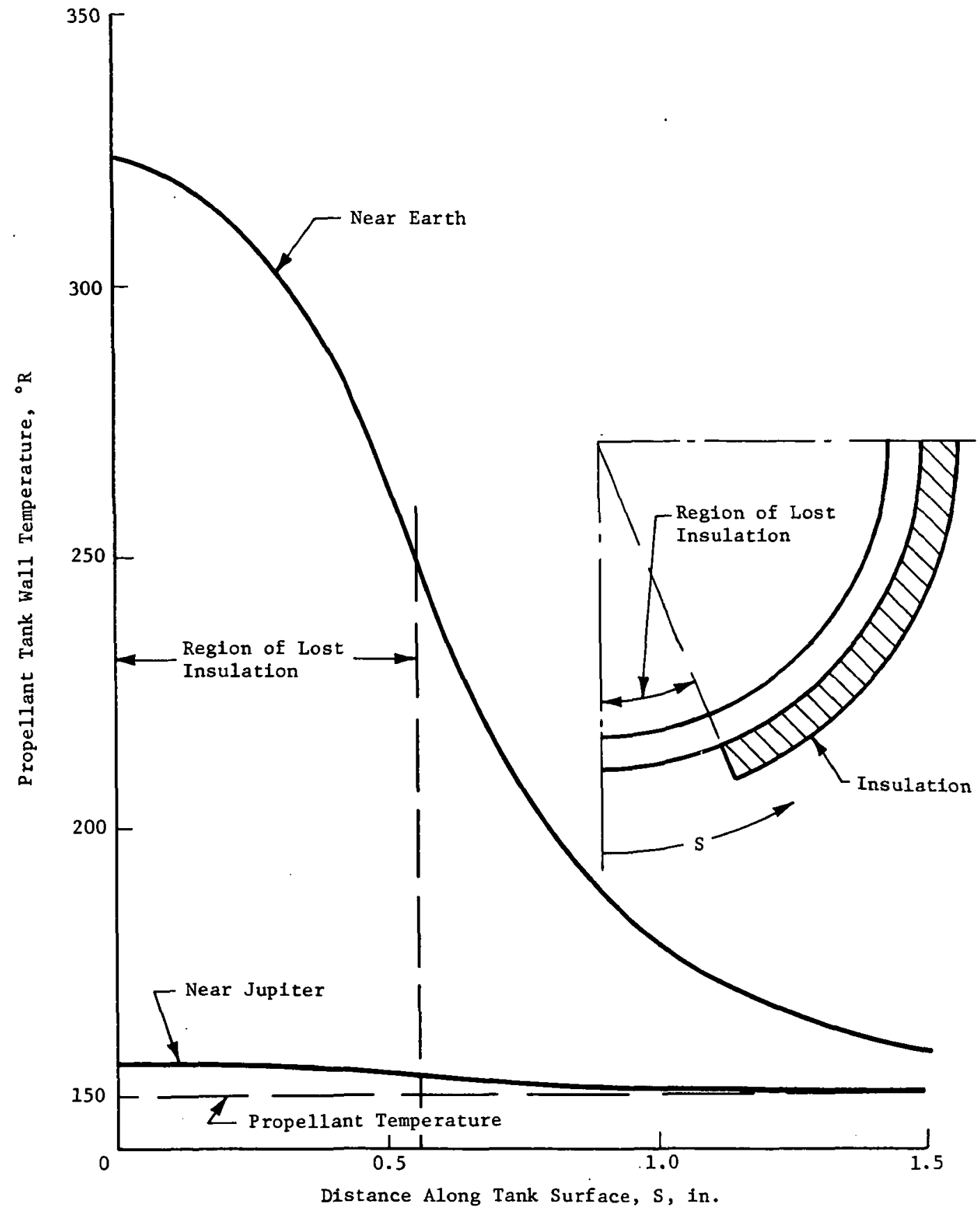

Fig. III-60 Propellant Tank Wall Temperature Profiles 
thermal control system. The thermal control system would reduce the severity of the temperature rise by rejecting at least a part of the entering heat.

The effect of time on propellant heating was also considered. It was assumed that all the energy entering the propellant tank through the exposed wall was absorbed by the propellant bulk. The propellant was assumed to be uniformly mixed so that no thermal stratification occurred. Near Earth, the following data were ass umed:

$\mathrm{q}_{\mathrm{S}}=430 \mathrm{Btu} / \mathrm{hr} \mathrm{ft}^{2}$, solar flux;

$\alpha=0.9$, absorptivity,

$\mathrm{M}=9401 \mathrm{~b}_{\mathrm{m}}$, propellant mass,

$\mathrm{C}_{\mathrm{P}}=0.362 \mathrm{Btu} / \mathrm{lb}_{\mathrm{m}}{ }^{\circ} \mathrm{F}$, propellant specific heat.

Under these conditions, the propellant bulk temperature would rise $1.14^{\circ} \mathrm{R}$ per hour of exposure to the sun. From the mission criteria, the maximum allowable time that the propulsion system may be in the sun, at one time, is 75 minutes. In this time, the propellant temperature would rise only $1.42^{\circ} \mathrm{R}$.

Near Jupiter, the solar constant approaches $16 \mathrm{Btu} / \mathrm{hr} \mathrm{ft} \mathrm{ft}^{2}$ and the propellant mass varies from $4901 \mathrm{~b}_{\mathrm{m}}$ prior to the plane change at apoapse to $1.4 \mathrm{lb}$ prior to the final orbit trim burn. Under these conditions, the bulk temperature change rate would be as low as $0.08^{\circ} \mathrm{R}$ per hour of solar exposure to as high as $10.3^{\circ} \mathrm{R}$ per hour of solar exposure.

Based on this lumped analysis and the maximum time that the propulsion system may be exposed to the sun, the loss of $1 \mathrm{sq} f t$ of insulation from the Flox tank does not appear to present a major problem. The thermal control system should provide a mitigating effect. In addition, rotation of the spacecraft to shade the propulsion system should be accomplished in the event of an unexplained pressure rise in the Flox tank. This maneuver will remove the heat load while still allowing the exposed tank wall to radiate to space. These results are applicable to the oxidizer tank in either the Flox/MMH system or the $\mathrm{F}_{2} / \mathrm{N}_{2} \mathrm{H}_{4}$ system. Similar results would be expected in both tanks of an $\mathrm{OF}_{2} / \mathrm{B}_{2} \mathrm{H}_{6}$ system. 
In conclusion, an anomalous operation only should result from the loss of $1 \mathrm{sq} \mathrm{ft}$ of insulation from the tank. It is felt that no catastrophic failure would result if the maximum exposure time of the bare tank wall to the sun is held to 75 minutes. This applies to both near-Earth and near-Jupiter operation. However, exposure for even this amount of time could result in problems during the final steps in the mission. The worst-case situation would occur just before the final orbit trim burn. The magnitude of the problem would depend to a major extent upon the location of the bare tank wall. Minimizing the solar exposure time may be required in this instance. Further evaluation with a detailed thermal model is indicated. 
C. SYSTEM FABRICATION, ASSEMBLY AND TANK INSTALLATION

As part of the comparative evaluation of the various candidate propellant acquisition concepts, the ability of each candidate to satisfy the required fabrication, assembly, and tank installation criteria was evaluated. This effort included identification of applicable assembly and tank installation procedures, evaluation of joining techniques, investigation of ground cooling coil integration in each candidate concept, and an investigation of the impact of loading and handling requirements.

1. Applicable Assembly and Installation Procedures

Three assembly and tank installation techniques were identified as possible methods for evaluation. Each of the candidate concepts could employ at least one of these techniques. The first technique required that the candidate devices be totally assembled outside of the tank and then installed in the tank by insertion through a tank access port. The base of the acquisition device would either be part of the tank outflow port assembly or so designed so that it can be mechanically attached to the outflow port assembly after device installation. Therefore, with this technique final tank closure would consist of bolting the outflow port assembly to the tank access port flange. With this technique, the device could also be removed.

The second technique would allow the acquisition device parts to be inserted through the access port and assembled inside the tank. The acquisition device could be joined either inside the tank or during insertion, depending on the specific details of each particular device. However, the devices could not be removed with this technique unless mechanical fastening methods were used. Final tank closure would be accomplished by bolting of the outflow port to the tank flange similar to the first technique.

For the third technique, no tank access port would be employed. The propellant tank and the candidate acquisition device would be assembled together as an integral unit. Final tank closure would be through a closure weld of the propellant tank.

The first assembly and tank installation technique would be the preferred procedure because of its greater flexibility. For the Category C Fruhof devices, at least two of the concepts (standpipe and post) appeared capable of fitting through a tank access port. However, for both the vaned concepts and the post-with-fingers concept, the ability of these devices to be inserted through the tank access port depends on whether or not the vanes or fingers could 
be elastically bent so they could pass through the access port. For the vaned Fruhof concepts, bending elastically or without damage, amounts to having the vanes rolled into a diameter smaller than the access port diameter (Fig. III-61).

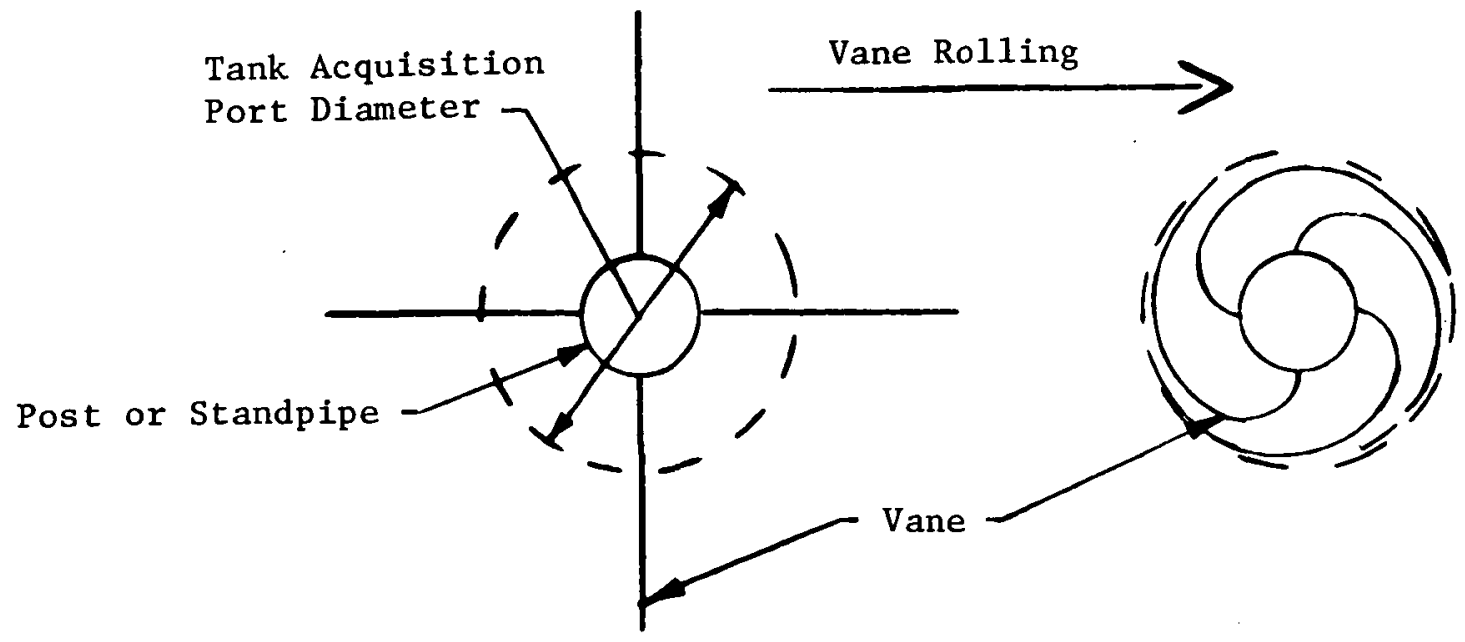

Fig. III-61 Installation of Four Vaned Acquisition Device by Rolling

To ascertain whether the vanes can be rolled into such a diameter, a structural analysis was conducted on the elastic bending capability of vanes. For the structural analysis, a pure bending model was employed. Therefore, for totally elastic behavior:

$\sigma=E \varepsilon$

where

$\sigma=$ stress;

$E$ = modulus of elasticity;

$\varepsilon=$ strain;

The strain was related to the curvature produced in the vanes due to rolling by:

$\varepsilon=\frac{\mathrm{tK}}{2}$ 
where

$K=$ curvature,

$t=$ thickness of vane.

Therefore:

$\sigma_{\max }=\frac{E t}{2} K_{\max }$

As the above equation shows, the maximum stress in the vane depends on the maximum rolled curvature. Therefore, with the allowable maximum stress known, the maximum rolled vane curvature for a particular vane thickness can be determined. To relate the maximum vane curvature to the size of the tank access port, a computer program was developed. This program solves the nonlinear elastic stress equations for thin vanes subject to two constraints. First, the outer edge of the vane must be tangent to a circle representing the access port. Second, the inner edge of the vane must be perpendicular to another circle representing the standpipe or post concentric to the first circle (see Fig. III-61). For any point on the resulting vane shape, the program also automatically computes vane curvature and internal forces. Due to the constraint of tangency to the outer circle the computer program's solutions are only valid when the ratio $L / \Delta R \geq 5 / 3$, where $L$ is defined as the maximum vane width and $\Delta R$ is $R-r$. $R$ is the tank access port radius while $r$ is the standpipe or post radius. For $L / \Delta R<5 / 3$, bending stresses are not severe; therefore the vanes can be readily installed. Figure III-62 shcrs the relation between $r, R$, and $K_{\max }$ obtained from the computer program. As $r$ approaches $R, K_{\max }$ approaches infinity.

From Figure III-62 and Equation [III-47] vane thickness was obtained as a function of standpipe of post diameter for the specific tank access port diameter of 9 in. Twelve representative vane materials were considered. These were:

$6 \mathrm{Al}-4 \mathrm{~V}$ Ti annealed;

$5 \mathrm{Al}-2.5 \mathrm{Sn} \mathrm{Ti}$ annealed;

7075-T6 Al;

$304 \mathrm{~L}$ annealed stainless;

$304 \mathrm{~L} 60 \%$ cold reduced stainless;

Inconel 718 heat treated and aged; 1100-0 Al.
17-4 PH (H1100) stainless;

$\mathrm{Be}-\mathrm{Cu}$ annealed;

Be-Cú cold reduced;

2219-T87 Al;

6061-T6 Al; 


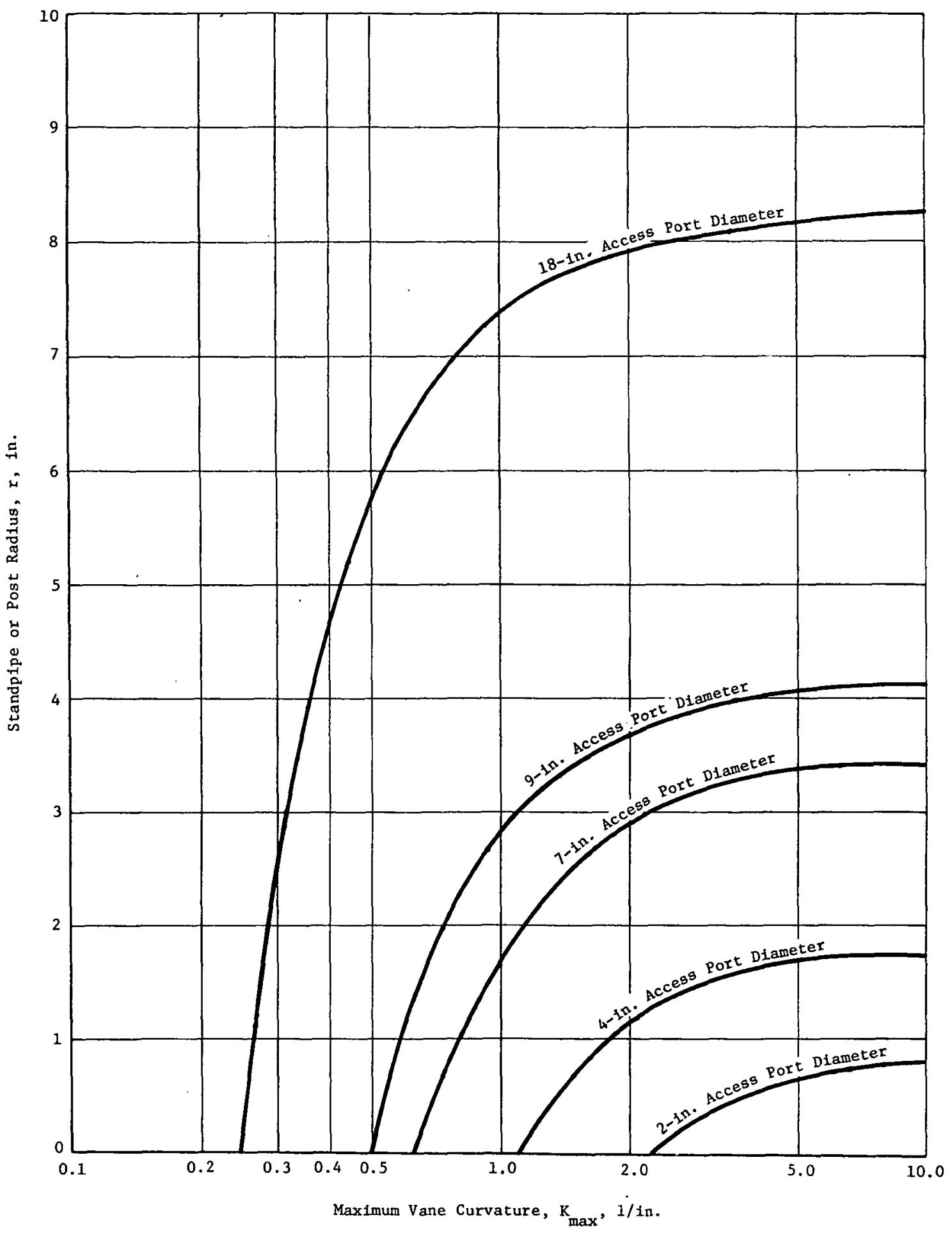

Fig. III-62 Maximum Vane Curvature Occurring during Instailation of Acquisition Device 
For each of these materials, the value of $\sigma_{\max }$, used in Equation [III-47], was determined by taking the material elastic limit and applying a $10 \%$ safety factor.

The results are presented in Fig. III-63. As can be seen, the ability of the large vanes to be inserted through the 9-in. tank access port is very dependent on the vane material and the standpipe or post diameter.

A structural analysis was also performed on the post-with-fingers concept to determine if the fingers could bend without damage (elastic bending) during installation through the tank access port. For this analysis an approximate thin beam, pure bending model was employed. Figure III 64 describes the approximations made in the analysis. It was assumed that the fingers have a straight portion in their shape near their base. This straight portion was assumed so that the thin beam bending model could be used for the analysis. It was also assumed that the fingers would have to be bent through the angle $\theta$ (defined in Fig. III-64) to accommodate tank installation. Therefore, the maximum stress developed in the fingers when bent through $\theta$ can be approximated from thin beam bending theory by (Ref III-17):

$\sigma_{\max }=\frac{E t}{L} \tan \theta$

where:

$t=$ finger thickness;

$\sigma_{\max }=\operatorname{maximum}$ elastic stress;

$E$ = modulus of elasticity,

and where $\theta$ and $L$ have been defined in Fig. III-64. Using Equation [III-48], finger thickness was calculated for two specific values of $\theta$. L was assumed equal to the radius of the Flox.tank ( $\mathrm{L}=17.75 \mathrm{in.}$ ). Table III-13 prësents these finger thicknesses for three reference materials based on a $\sigma_{\max }$ equal to $90 \%$ of the elastic limit. 


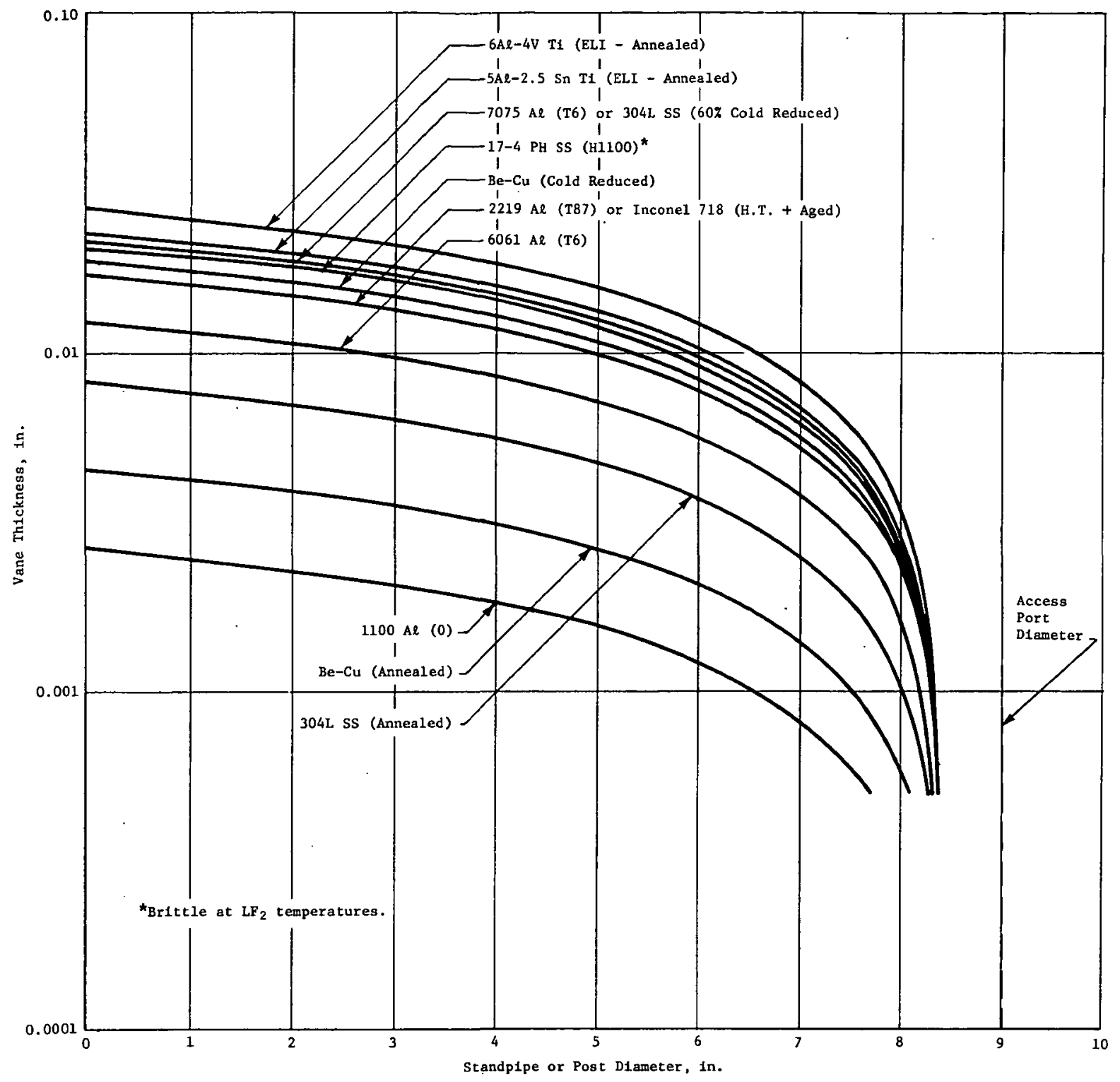

Fig. III-63 Marimm AlLowable Vane Thiokness to Install Acquisition Device 


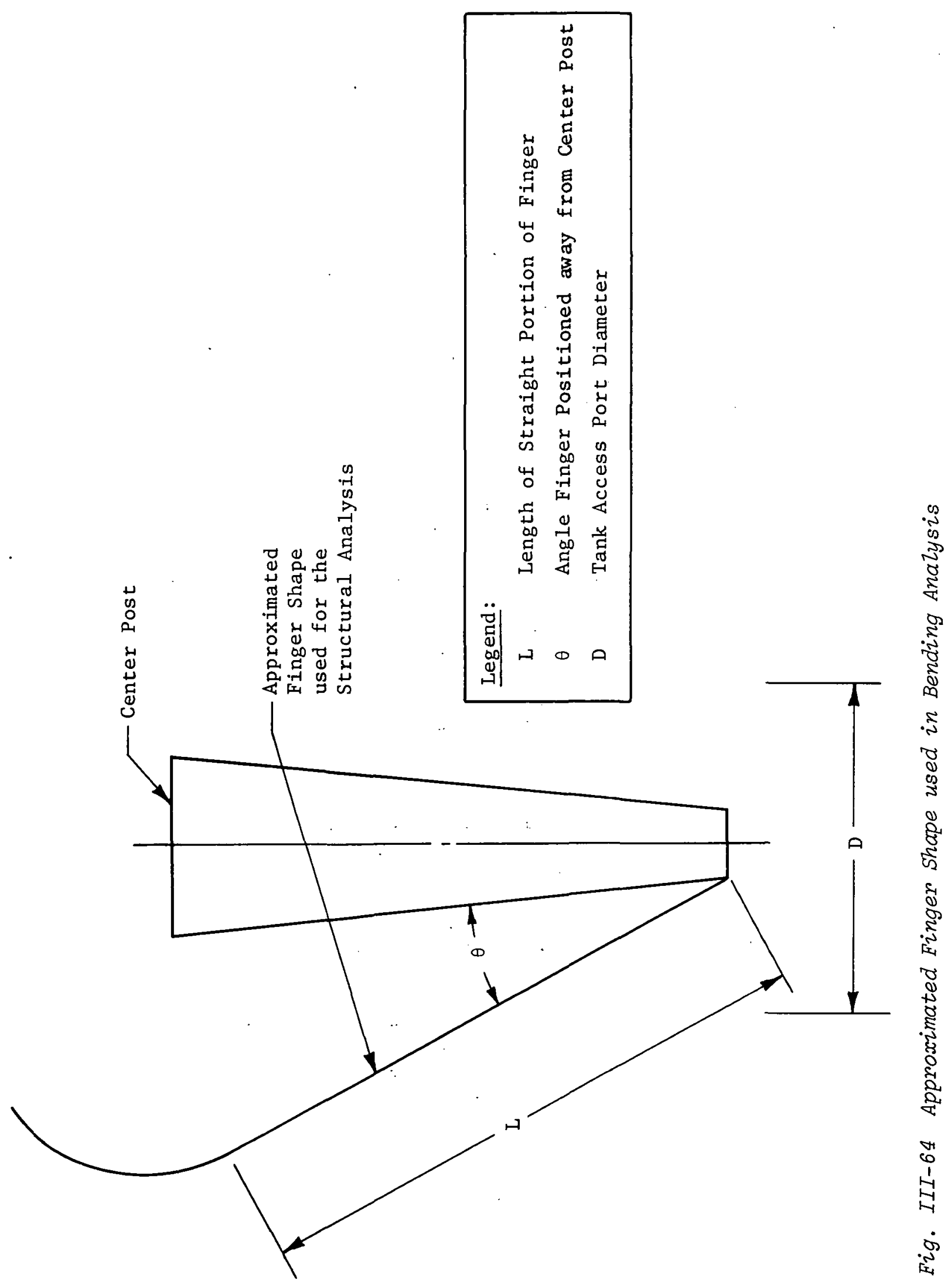

III-109 
Table III-13 Maximum Finger Thickness (in.)

\begin{tabular}{|l|l|l|}
\hline Material & $\theta=30^{\circ}$ & $\theta=45^{\circ}$ \\
\hline $2219 \mathrm{~T} 87 \mathrm{Al}$ & 0.130 & 0.075 \\
$304 \mathrm{~L}$ SS Cold Reduced & 0.165 & 0.094 \\
$6 \mathrm{Al}-4 \mathrm{~V} \mathrm{Ti}$ & 0.202 & 0.117 \\
\hline
\end{tabular}

On the basis of these finger thicknesses it would appear possible for the fingers concept to use the first installation procedure since a $30^{\circ}$ bending angle would be greater than would actually be needed for installation. A finger thickness between 0.13 and 0.2 in. would be reasonable values for design purposes.

\section{Joining Processes}

A major factor influencing selection of an assembly and tank installation procedure was the type of joining processes that could be used. Three general joining methods are available: welding, brazing, and mechanical fastening. Three welding techniques are: resistance, fusion and electron beam welding (EBW). Based on a previous study (Ref III-1), resistance welding is not applicable for any of the candidate concepts because of inherent contaminated trap areas. Fusion welding can only be used on relatively massive parts where material warpage is not a factor. EBW is mainly applicable to thin or delicate parts because the heataffected zone is kept to a minimum. Brazing seems applicable but strongly depends on the type of materials to be used for the candidate devices and the propellants to be used (Ref III-1). Mechanical fastening in general, like resistance welding, contains inherent contaminant trap areas (Ref III-1) although certain types of mechanical fasteners may be used.

Considering the applicable foining methods from the standpoint of propellant compatibility, further restrictions arise. For either MMH or $\mathrm{N}_{2} \mathrm{H}_{4}$, service compatibility considerations dictate the use of titanium (Ref III-18) as the material of construction for both the propellant tanks and the acquisition devices (baseline tanks are to be $6 \mathrm{Al}-4 \mathrm{~V}$ titanium). As far as fusion or EBW welding is concerned, both of these methods can be used on titanium. Brazing also seems applicable for the fuel tanks. Only two techniques for brazing titanium are apparently available. One uses a $48 \mathrm{Zr}-4 \mathrm{Be}$ braze alloy; the other uses 3003 aluminum. As far as braze integrity is concerned the $48 \mathrm{Zr}-4 \mathrm{Be}$ alloy appears excellent. Also, slight oxide coatings can be tolerated with this technique (Ref III-1). Zirconium has been rated as a compatible material for use 
with $\mathrm{N}_{2} \mathrm{H}_{4}$ or MMH (Ref III-18). Beryllium seems also to be compatible with hydrazine-type fuels even though no specific compatibility data exist. Due to beryllium's atomic structure it would theoretically not be a catalyst for decomposition of hydrazine fuels (Ref III-18). One problem that may restrict the use of $48 \mathrm{Zr}-4 \mathrm{Be}$ titanium braze is beryllium poisoning. Beryllium is very toxic. However, with proper precautions the poisoning hazard can be eliminated.

The 3003 aluminum braze appears even more applicable than the $48 \mathrm{Zr}-4 \mathrm{Be}$ braze. Aluminum, like titanium, is highly compatible with MMH and $\mathrm{N}_{2} \mathrm{H}_{4}$ (Ref III-18); therefore, there should not be any compatibility problems with this braze alloy. Based on preliminary data, the integrity of 3003 aluminum brazed titanium joints appears excellent. Also, any titanium alloy can be brazed to any other titanium alloy without lowering the quality of the braze joint. However, a completely clean, oxide-free surface is required for this procedure (Ref III-1).

The use of mechanical fasteners for assembly of the candidate concepts to be used inside either the $\mathrm{N}_{2} \mathrm{H}_{4}$ or $\mathrm{MMH}$ tanks, is limited to those fastening techniques that do not create any contaminated trap areas. Trap areas formed when parts are mechanically assembled, even if in a clean condition during assembly (parts cleaned before assembly and assembled under clean room conditions), can entrap either test fluids used in qualification tests or cleaning fluids used in final tank system cleanings. These types of fluids may accelerate fuel decomposition or degrade fuel contact angle. Therefore, any mechanically fastened parts must be fastened so that all contacting part surfaces are completely sealed off from contact with the propellants. This limits mechanical joining methods to those using crushable gaskets or seals so that sealing can be accomplished. Gasket materials compatible with either $\mathbb{M}$ or $\mathrm{N}_{2} \mathrm{H}_{4}$ are limited to Teflon, polyethelene, and soft aluminum.

The preferred joining procedures for the fuel candidate concepts are either EBW or fusion welding; or $48 \mathrm{Zr}-4 \mathrm{Be} \mathrm{Ti}$ or $3003 \mathrm{Al}$ brazing. However, crushable gasket mechanical fastening could probably be used if absolutely necessary.

For either the Flox or $\mathrm{LF}_{2}$ tanks, greater restrictions on acquisition device joining methods are imposed than those found with either the MMH or $\mathrm{N}_{2} \mathrm{H}_{4}$ tanks. For Flox or $\mathrm{LF}_{2}$, material compatibility dictates the use of either 300 series stainless steel or aluminum (baseline tanks are 2219-T87 aluminum) as a material of construction (Ref III-18). Fusion welding is applicable to both 300 series stainless as well as most of the aluminum alloys (1100, 
6061, and most of the 2000 series are weldable, 7075 is not). EBW can also be used on 300 series stainless steel. However, the use of EBW on aluminum alloys is more restricted. Most alloys of aluminum are subject to cracking with EBW. This problem seems especially severe with the 2000 series (Ref III-19). Therefore, if $E B W$ can be used at all with aluminum alloys it would probably be more applicable with alloys such as 1100 or 6061 .

Brazing of aluminum alloys is not applicable for Flox or $\mathrm{F}_{2}$ service and questionable for 300 series stainless steel alloys. This is because all aluminum brazing alloys are incompatible with Flox or $\mathrm{F}_{2}$ and stainless steel brazing alloys might cause galvanic corrosion problems with Flox for $\mathrm{F}_{2}$. All braze alloys used for aluminum brazing have a high silicon content. Alloy 718, for example, contains $13 \%$ silicon. In addition, for most of these brazes a practically pure silicon flux is used (Ref III-1). If silicon is present in a material in a quantity greater than $1 \%$, fluorine or flox will react with the silicon to form $\mathrm{SiF}_{4}$ (Ref III-20). Instead of adhering as a protective film, the $\mathrm{SiF}_{4}$ will vaporize if exposed to either $F$ lox or $F_{2}$ gas above $350^{\circ} \mathrm{R}\left(\mathrm{SiF}_{4}\right.$ melts at $321^{\circ} \mathrm{R}$, Ref III-20). $\mathrm{GF}_{2}$ at room temperature will be used to passivate the $\mathrm{F}_{2}$ or Flox tanks prior to propellant loading. Therefore, any brazed aluminum joints will be attacked and weakened by the passivation. Also, even at $\mathrm{LF}_{2}$ or liquid Flox temperatures the integrity and protective nature of the solid $\mathrm{SiF}_{4} \mathrm{film}_{\mathrm{m}}$ is questionable (Ref III-18).

For the brazing of 300 series stainless steel alloys, four types of filler metals or brazing alloys exist (Ref III-21). These alloy types are designated as $\mathrm{BAg}, \mathrm{BNi}, \mathrm{BCu}$, or $\mathrm{BAu}$. The second symbol designates the main metal component of each alloy type. For instance, the $\mathrm{BCu}$ types have as their major component copper, while BNi alloys have nickel. For the BAg or silver-alloy types, the other metal constituents used are $\mathrm{Cu}, \mathrm{Zn}, \mathrm{Cd}, \mathrm{Ni}$, and $\mathrm{Sn}$. Therefore, on the basis of the compatibility of these metals with $\mathrm{F}_{2}$ (Ref III-18), the BAg filler metals in themselves should be compatible with either $\mathrm{LF}_{2}$ or Flox. BCu and BAu alloys should also be compatible. The BCu alloys are practically pure copper and the one BAu alloy contains $80 \% \mathrm{Au}$ and $20 \%$ nickel; all compatible with $\mathrm{F}_{2}$. However, for the nickel brazing alloys (BNi), either 3 to $4 \%$ silicon or boron, or as much as $10 \%$ phosphorus is used as one of the other metal constituents. As stated earlier braze alloys containing over $1 \%$ silicon are incompatible with $\mathrm{F}_{2}$ because $\mathrm{SiF}_{4}$ is volatile at $\mathrm{GF}_{2}$ passivation temperatures. Both boron and phosphorus also form low melting point fluorides $\left(\mathrm{PF}_{3}\right.$,

III -112 
$\mathrm{PF}_{5}$ and $\mathrm{BF}_{3}$ have melting or sublimation points below $-70^{\circ} \mathrm{F}$, Ref III-18). Therefore brazing alloys containing large enough quantities $(>1 \%)$ of these metals are probably incompatible for service with either Flox or $\mathrm{F}_{2}$. This leaves only the silver, copper, and gold brazing alloys as possible $\mathrm{F}_{2}$ compatible filler metals for stainless steel.

Although the $\mathrm{BAu}, \mathrm{BCu}$, and $\mathrm{BAg}$ braze alloys are in themselves probably compatible with either Flox or $F_{2}$, when combined with 300 series stainless steel, higher corrosion rates may be encountered for these alloys. Fairly recently galvanic corrosion couples have been detected with $\mathrm{LF}_{2}$ (Ref III-22). In compatibility tests, various metal couples were exposed to $L F_{2}$. The results of these tests showed higher corrosion rates for the alloys used in testing than those usually reported for these alloys when tested uncoupled or alone in $\mathrm{LF}_{2}$. Considering these results, galvanic corrosion effects will definitely be present for metal couples exposed to $\mathrm{LF}_{2}$ if these couples are composed of metals differing in electrode potential by a fairly large amount. The following relative galvanic corrosion series was obtained based upon the metals tested ( $\operatorname{Ref}$ III-22):

\begin{tabular}{l|l}
$304 \mathrm{~L}$ stainless steel & $\begin{array}{l}\text { Increasing } \\
\text { Copper }\end{array}$ \\
Nickel-200 & Anodic \\
Silver & Behavior \\
$1100-0$ aluminum &
\end{tabular}

On the basis of this series, the use of either BAg or BAu brazing alloys with 300 series stainless steel is not recommended because of the potential of high corrosion rates. The use of $\mathrm{BCu}$ alloys is questionable. Over a period of 10 years any brazed stainless steel joints might be attacked and thus weakened by increased corrosion due to galvanic cells set up between the stainless steel and braze alloy.

The use of mechanical fasteners for $F$ lox or $F_{2}$ system components should be avoided because most mechanical fastening means create contaminant trap areas. As stated earlier, trap areas, even in a clean condition after device assembly, can present problems because contaminants can be entrapped when qualification tests or final propellant system cleanings are performed. For hydrazinetype fuels, these contaminants may degrade propellant contact angle or accelerate fuel decomposition. These are serious problems but 
not necessarily catastrophic. However, with the fluorine-based propellants, these contaminants may cause ignition of the entire propellant tank and its components. Therefore, based on these considerations, the only mechanical fastening methods that might be applicable for fluorine service are those that do not create any trap areas. This limits mechanical fastening techniques to those using crushable gaskets or seals so that all contacting component surfaces can be completely sealed off from propellant contact. These sealed-type fastening techniques have already been recommended as the only mechanical joining procedures applicable for $\mathrm{N}_{2} \mathrm{H}_{4}$ or MMH service earlier.

Although crushable gasket-type fastening methods can probably eliminate contaminant trap area problems, there are problems or limitations associated with their use with fluorine-based propellants. One such limitation is the type of gasket or seal material that can be used. Based on material compatibility considerations, no nonmetal seals can be used (Ref III-18). Therefore, only soft metal seals or gaskets can be used. Based on fluorine tests at Martin Marietta over the past decade, either soft copper or aluminum seems applicable.

A far more severe limitation on the use of a crushable gasket or seal-type fastening technique with $\mathrm{LF}_{2}$ or Flox, arises from the fact that both $\mathrm{LF}_{2}$ and Flox are cryogens. Unless properly designed, the gasket or seal material could shrink away from its seating surface because of thermal contraction during propellant loading and tank chilldown. If this happens, the $\mathrm{LF}_{2}$ or Flox will leak with catastrophic results. To prevent this, the gasket, fastener, and even the seating surfaces must not have their thermal expansion coefficients differ by too large an amount. Therefore, if the tank and acquisition devices are aluminum, a soft aluminum gasket or seal is advisable.

Welding seems to be the most reliable joining process available for fluorine-based propellant components. However, adequate mechanical fastening techniques can probably be developed for $\mathrm{F}_{2}$ or Flox service if necessary; although the reliability of such techniques for long-term use is questionable.

3. Recommended Concept Assembly and Installation

Based upon the above discussion, recommended joining and tank installation procedures were determined for each of the seven candidate propellant acquisition concepts. These recommendations are discussed in the following paragraphs.

III-114 
a. Compartmented Tank - For this concept the third or integral procedure seems the only applicable tank installation technique. To accomplish the first or preferred tank installation procedure, the compartmented tank acquisition concept would have to have its capillary barriers structured to act as umbrellas. They could then be inserted through the tank access port and opened up for positioning and attachment. Structuring the barriers to act as umbrelilas would require the use of mechanical joints which are undesirable from the standpoint of contaminant trap areas. Such joints would also be susceptible to metal-to-metal rubbing during the mission, which could ignite the Flox or $F_{2}$ tanks (nonmetal spacers or washers can not be used with fluorine-based propellants, Ref III-18). Regardless of these considerations, once the barriers have been inserted into the tank, the attachment of the umbrellatype capillary barriers to the tank, would be difficult if not impossible. An upper barrier could possibly be bolted to a flange provided in the tank wall through the tank access port, but attachment of the bottom barrier assembly or trap assembly with its liner would be nearly impossible because of the lack of space.

For the second installation technique, segments of the capillary barriers and liner could possibly be inserted through the tank access port. However, to then join them together inside the tank would either require welding or mechanical fastening. Brazing would not be possible because the tank would be annealed. Welding through the access port would not be possible because of space limitations. Although mechanical fastening would be extremely difficult, it could be done. Even if the device can be assembled, the problem still remains of how to attach the device to the tank. The third installation procedure does not present any of the problems associated with the other two techniques. Installation would be accomplished by having the barriers and liners,an integral part of the tank structure (for a more detailed description of such a design see Ref III-1). Welding would be either by fusion or electron beam. Fuel tank subassemblies could be brazed as long as such assemblies are not an integral part of the tank's pressure shell (brazing will anneal and thus weaken components). Brazing would not be applicable for either the Flox or $F_{2}$ tanks as discussed earlier.

b. Cruciform - The cruciform is capable of using the first or preferred tank installation technique. However, mechanical fastening techniques would have to be used. The installation procedure would be as follows. The cruciform would be assembled either by welding, brazing, or machining out of one piece. After assembly, 
it would be inserted into the tank through the access port. It would be attached to the top of the tank by a sealed type of mechanical fastening joint such as a bolt flange with a gasket. This could be built into the top of the cruciform before installation. After installation and attachment, the outflow port assembly would be bolted to the base of the cruciform as this assembly is being bolted to the tank. Having the base of the cruciform as part of the outflow port would not be practical because there would be little clearance for attaching the cruciform to the top portion of the tank.

The cruciform is capable of fitting through the tank access port, thus assembly within the tank is not needed. Therefore, the second installation procedure would not be applicable to the cruciform concept. However, the third or integral procedure would be applicable if an all-welded design became necessary. As stated earlier, an all-welded design is usually preferred for fluorinebased propellant systems. Thus, the third installation procedure might be more desirable for the Flox or $\mathrm{LF}_{2}$ cruciform concepts.

c. Post or Standpipe - For both of these concepts the first installation procedure can be used as long as the outer diameter of the devices is not larger than the diameter of the tank access port. Also, since the top of these devices is not attached to the tank, as is the cruciform concept, the outflow port assembly can be part of or welded to the base of the standpipe or post. Space is not needed to attach the device to the tank as it is being installed. However, to install the communication channels, mechanical fastening seems to be required. To be able to install and remove the communication channels requires attaching these channels to the tank wall by mechanical means. Bolts with washers or gaskets around their heads might be attractive. With this type of fastener, sealing around the bolt head might be possible, thus eliminating any contaminant trap areas. Therefore, with either the post or standpipe designs, the first installation procedure can be used with mechanical fastening of the communication channels.

The second installation procedure was not considered because both the post and standpipes can be inserted through the tank outlet in an assembled form. However, if an all-welded design becomes necessary (communication channels welded to tank wall), the third or integral procedure could be used with the post or standpipe concepts. 
d. Post with Fingers - For this concept the discussion about the installation and assembly procedures for the post and standpipe designs also applies. However, there would be one difference in the first installation procedure for the fingers concept. To fit this device through the tank access port requires that the fingers bend elastically toward the post during device insertion. This seems to be possible, thus making the first installation procedure applicable. Welding of the fingers to the base of the post would probably be the fingers joining process.

e. Vaned Concepts - For these concepts, the ability to use the first or preferred tarik installation procedure depends on two considerations. The first is whether the vanes can be rolled into a diameter smaller than the tank access port diameter. The vane rolling analysis presented earlier showed that the ability of the vanes to do this depends on the material used, the minimum vane thickness allowable, and the post or standpipe diameter used. Figure III-63 shows that practically any material can be used if the vane is thin enough. However, one additional restriction on vane thickness, beside manufacturability, is imposed by the mission requirement of a 10-year life. The restriction is one of possible fluorine corrosion of the vane material. Extensive corrosion data are not now available. Therefore, the vanes should be as thick as possible. Since only aluminum and 300 series stainless steel seem applicable construction materials with $\mathrm{F}_{2}$ (Ref III-18), this would probably limit the vane material to either $304 \mathrm{~L}$ cold reduced stainless steel or 2219-T87 aluminum. If these materials were used, there would seem to be no minimum vane thickness problems for the pure-vaned concept. However, based on Fig. III-63, for the standpipe concept, standpipe diameters would have to be kept below 5 in. to keep vane thickness at least 0.010 in. thick. For use with either $\mathrm{MMH}$ of $\mathrm{N}_{2} \mathrm{H}_{4}$ the vaned concepts could have their vane thickness less than those needed for either Flox or $\mathrm{LF}_{2}$ because no corrosion problems would exist. As discussed previously, titanium would probably be the vane material for either MMH of $\mathrm{N}_{2} \mathrm{H}_{4}$ because of compatibility considerations.

The second consideration is the type of procedure to roll the vanes into the required installation diameter. Some sort of rolling tool would have to be used. During device installation, this tool would have to be inserted into the tank along with the device to keep the vanes in the rolled position. To remove this tool after the acquisition device has been inserted into the tank and its vanes unrolled, would require the rolling tool to either slip over the base of the acquisition device or to telescope so that is could be taken apart and removed from around the base. The precise design will influence what type of joining operations can be used to 
satisfy the first installation procedure. If the rolling tool is of a telescoping design, the outflow port assembly can then be part of the acquisition device base; welding or brazing of the outflow port assembly to the base of the device could be done. However, the design of the vaned acquisition devices would have to allow for enough space to remove the tool. If the rolling tool is not designed to telescope, the outflow port can not be part of the acquisition device base because of inadequate clearance. After the device is installed, the rolling tool would have to be removed by slipping over the base of the device. The outflow port assembly would then be mechanically attached to the acquisition device base after tool removal.

As the above discussion shows, the ability of the vaned concepts to use the first tank installation technique depends on whether the vanes can be rolled into the required installation diameter, and the methods by which this rolling is done. Assuming that the vanes can be rolled into the required diameter, the choice of using either a welded or brazed assembly or mechanically fastened assembly depends on the type of rolling tool design which is applicable. Regardless, the device's communication channels must still be attached by mechanical fastening, as discussed under the post and standpipe concepts. Therefore, some form of mechanical fastening must still be used with the vaned concepts for these concepts to use the first installation procedure. To eliminate all mechanical fastening, the third or integral installation procedure would have to be used for the vaned concepts.

\section{Cooling Coil Integration}

A requirement that the Flox and $F_{2}$ propellant acquisition devices must meet is the integration of a $\mathrm{LN}_{2}$ ground-hold cooling coil to be located within the propellant tank. This requirement also applies to $\mathrm{OF}_{2}$ and $\mathrm{B}_{2} \mathrm{H}_{6}$ tanks. Cooling must be maintained on the ground during propellant loading and subsequent ground operations before launch to prevent oxidizer venting. The ground cooling coils must not interfere with the normal operation of the acquisition device. For all seven candidate concepts, integration with an $\mathrm{LN}_{2}$ cooling system is possible. However, for at least some of the concepts, cooling coil integration may impact the type of tank installation procedure that can be used to install the acquisition device. 
a. Compartmented Tank - For the compartmented tank concept, the $\mathrm{LN}_{2}$ cooling coil can be attached to the lower capillary barrier as structural members. This attachment will not affect either the operation or installation of the concept. Since the third or integral device installation procedure would probably be used with the concept, no major impact would be imposed on this tank installation procedure by having the cooling coils part of the lower capillary barrier. The cooling coil and lower capillary barrier assembly would simply be welded into position as if there were no cooling coils. Inflow and outflow tubing to the coil could be provided through the tank outflow port during assembly.

b. Cruciform - For the cruciform concept, the only practical way of integrating a coil would be to design the cruciform to operate as the cooling coil itself. Wrapping a cooling coil around the cruciform in either an attached or free-floating manner may reduce expulsion efficiency, increase flow losses, or create contaminant traps. However, cooling channels in the cruciform vanes would not introduce such problems. $\mathrm{LN}_{2}$ flowing through these channels could provide the required ground cooling. The vane channels could be connected by manifolds located at the base and top portion of the cruciform. With this design no major impact would be imposed on either the first or third tank installation procedure. of course, the exact sizing of the channels would depend on the cooling requirements defined by thermal analysis.

c. Post and Standpipe - For both the post and standpipe concepts, the most practical way of integrating a cooling coil would be to either wrap the coil around the outer surface of the devices or incorporate cooling channels within the devices. These two methods provide a sound acquisition system design from the standpoint of structural integrity and, at the same time, have minimum influence on the fluid dynamic design. Installation of a cooling coil in the bottom of the tank, but not in contact with the post or standpipe, could also be a workable design approach. However, support of the coil would be difficult and presentia structural design problem. Furthermore, the arrangement and location of the coil could degrade the fluid dynamic performance of the device if.the design is not developed carefully. Location of the cooling coil in the top of the tank in the ullage region is impractical because of poor cooling heat transfer during ground operations and because of the effect the coil would have on ullage orientation and shape under zero-g condition. 
The integral.cooling channel methods are preferred over the wrapped coil. With a wrapped coil there is a possibility of creating propellant trap areas between coil.windings, which may hold some propellant away from the tank outlet. The small areas would also be difficult to clean and passivate. With the integral method no such operating problems would exist.

Another factor to consider when comparing the two methods is the amount of cooling effectiveness available. The: cooling effectiveness depends on the amount of surface area the post or standpipe affords. If this area is not sufficient to satisfy cooling requirements, the Flox or $\mathrm{LF}_{2}$ will not be properly cooled. Therefore, although these cooling coil integration methods may be attractive from the standpoint of noninterference with device.operation, they may.not satisfy cooling requirements. The post concept seems to be more of a problem in this regard, because its surface is less than that of the standpipe:

d. Post with Fingers -. For the post-with-fingers concept the discussion:for the post concept generally applies. However, with this concept there is one additional way of integrating a cooling coil into the design without affecting its operation. This method would be to design the fingers as the cooling coil. Each finger could be constructed to consist of an inflow and outflow set of tubes. These cooling tube fingers could be connected through the center post. With this type of design, adequate cooling would seem to be available to satisfy the cooling requirements. The only problem is that the bending capability of these cooling tube fingers may be-reduced preventing. use of the first.installation procedure.

e. Vaned Concepts - For the vanes alone, one way of integrating a cooling coil without creating either device installation or operating problems would be to locate the coil as a channel inside the center post. Upon $\mathrm{LN}_{2}$ circulation, the center post and its attached vanes would be chilled to $\mathrm{LN}_{2}$ temperatures. The vanes would' act as large fins to provide cooling. The major difficulty would be providing tube diameters inside the post that are sufficient to carry the required $\mathrm{LN}_{2}$ flow rate. An alternative integration scheme would be to design the vanes with cooling channels. However-vane stiffness would be increased and bending capability reduced so that the first installation procedure could not be used. To locate a coil around the device or coiled in the upper half of the tank would interfere with the device's normal operation. 
For the standpipe with vanes concept, integration of $\mathrm{LN}_{2}$ cooling channels within the standpipe appeared to be best. As stated previously for the vane concept, incorporation of a cooling coil either around or above the acquisition device would create operational problems, while inclusion of cooling tubes in the vanes would limit bending or rolling of the vanes and therefore, would modify tank installation procedures. Thus, the only integration scheme that appears reasonable and workable is to incorporate cooling channels in the body of the standpipe itself. Advantages to this approach are: (1) large areas for $\mathrm{LN}_{2}$ flow can be provided; (2) standpipe surface can be used for heat transfer; and (3) vanes provide fin surfaces for heat transfer.

f. Altemative Cooling Coil Integration Method - All of the cooling coil integration methods discussed previously were concerned with incorporating the cooling tubes physically into the design of the propellant acquisition device. Two reasons for this approach were to use a modular installation and assembly technique and to minimize the impact of propellant acquisition system requirements on tank design and assmebly. For Category C devices requiring propellant communication, an alternative procedure could be used in which cooling coils placed on the tank wall would be designed to also function as communication channels. The advantage to this approach is that the propellant acquisition device design would be simplified. Modular installation of the device could still be done. However, the cooling coils would have to be attached to the tank walls during tank assembly. Installation of the cooling coils by parts into a finished propellant tank with subsequent assembly inside the tank is impractical because of the inability to produce reliable joints. Furthermore, if the tube joints could be welded inside the tank, the cooling tubes could not be removed without permanently damaging them. Therefore, the alternative cooling coil system must be installed in the propellant tank during the manufacturing process.

5. Loading and Handling Considerations

Prelaunch operations associated with propellant loading and spacecraft mating with the launch vehicle can impact the design and operation of the propellant acquisition device. These operations are discussed in the following paragraphs.

a. Tank Cleanliness and Passivation - A prime consideration in propellant loading is the requirement for tank cleaning. This requirement is applicable to both fuel and oxidizer tanks. No contaminants should be present that would provide catalytic action for decomposition or would degrade surface tension or contact angle 
properties over the ten-year mission life. For the oxidizers, the contaminant problem is more acute because of their highly reactive nature. The influence of the cleaning requirement on the design of the acquisition system is to minimize the number of possible contaminant traps. As discussed previously, use of mechanical joining techniques is especially critical in this regard. In the case of Fruhof systems using vanes, spacing and small angles between the vanes are also possible trap areas. In compartmented tank systems parallel perforated plates for barriers provide contaminant traps.

After tank cleaning, passivation of the oxidizer tank is required. Acquisition systems having the most surface area or more complex geometry would be the most difficult to passivate. Vaned systems and compartmented tanks would, therefore, present a more difficult passivation problem than simple standpipe or post-with-finger concepts.

b. Loading Techniques - From two other Martin Marietta-conducted programs (Ref III-1 and III-23), two propellant loading techniques have been identified as applicable methods for the space storable propellants. The methods are designated as vented and nonvented processes. Both would require operation of the $\mathrm{LN}_{2}$ ground cooling system to precool the propellant acquisition and tank system before loading. The requirement to precool the acquisition system could create transient thermal stresses in the acquisition device that would significantly affect its mechanical design. The problem could be especially severe in the cruciform concept where both ends of the device are attached to the propellant tanks. Contraction of the cruciform would induce loads on the propellant tank unless the forward end of the device had a slip fit joint (undesirable from a cleaning and passivation standpoint). The compartmented tank concept would alșo be susceptible to similar thermal contractions and stresses if uniform cooling was not maintained during the cooldown period. One additional problem with the compartmented tank is the possibility of trapping gas in the propellant reservoir at the bottom of the tank. Of the three categories of devices, only the compartmented tank would be susceptible to gas entrapment. Category $B$ and $C$ devices now envisioned do not contain bubble strainers or other regions capable of retaining gas.

c. Handling Considerations - Results from a previous Martin Marietta program (Ref III-23) indicated that loading of fluorine-type propellants should be accomplished prior to spacecraft installation on the launch vehicle. Loading would actually be performed at a remote area away from the launch pad for safety reasons. After loading, the system would then be transported to and installed on the launch vehicle. During this time, the system would be subjected to transportation loads and possible tipping or tilting during mating.

III -122 
Category $\mathrm{B}$ or $\mathrm{C}$ devices are not sensitive to possible accelerations or loads that may occur during transit. Nor are they susceptible to propellant tank tipping or tilting during elevation and installation on the launch vehicle. Category A devices, however, could ingest gas in the propellant reservoir if the tanks were tilted beyond certain limits during installation. In addition, during transport from the loading area to the launch pad, the Category A devices would also be sensitive to a combination of propellant slosh induced by transport loads and tipping on the transport vehicle. 
As part of the evaluation of the candidate propellant acquisition concepts, an experimental program was conducted in the Martin Marietta drop tower. The program was conducted to verify analytical predictions, to obtain information that can on $1 y$ be roughly estimated analytically, and to provide an experimental comparison of the performance of each candidate concept.

Small-scale glass tanks, each containing a scale model of cne of the candidate acquisition devices and filled to the desired level with the tes: liquid, were subjected to the desired low-g conditions in the 2.1-sec free-fall facility. Data on the fluid behavior in the test environment was provided by high-speed motion pictures taken during each drop test. A total of 34 drop tests were accomplished with five or six devices being tested in each drop.

TEST PLAN

The experimental program was progressive in nature, with the devices tested and the test conditions being varied based on the information obtained from previous testing and analysis. The acceleration environment for most of the tests was the near zero-g provided by the drop capsule. For the six stability tests performed, spring motors were used to apply acceleration to the test fixture.

Conditions varied in the zero-g tests were fill level and the orientation of the device with respect to the initial one-g. Fill levels from 5 to $95 \%$ liquid volume were evaluated. Methanol was used as the test fluid. Orientations of $0^{\circ}, 90^{\circ}$, and $180^{\circ}$ were used. At $0^{\circ}$ the outlet is oriented downward. Conditions varied in the stability tests were the force of the spring motor, fill level, and orientation.

Six sets of devices were tested. Based on the results of tests with one set, devices were eliminated or modified and new devices were added to form a new set. Devices were also eliminated when sufficient information'had been obtained concerning their operation. A list of the conditions and the device set used for each test are 1isted in Table IV-1. The six device sets are illus: trated in Fig: IV-1 thru IV-6. With regard to the illustrations of the vane type devices, only the profile of two of the vanes is shown, rather than an actual side view. 
Table IV-1 Drop Tower Tests

\begin{tabular}{|c|c|c|c|c|}
\hline $\begin{array}{l}\text { Test } \\
\text { Number }\end{array}$ & $\begin{array}{l}\text { Device } \\
\text { Set }\end{array}$ & $\begin{array}{l}\text { Liquid } \\
\text { Volume, \% }\end{array}$ & $\begin{array}{l}\text { Orientation, } \\
\text { deg* }\end{array}$ & $\mathrm{g}$-Level \\
\hline 1 & A & 20 & 0 & 0 \\
\hline 2 & 1 & 85 & 0 & \\
\hline 3 & & 85 & 180 & \\
\hline 4 & & 95 & 0 & \\
\hline 5 & & 95 & 180 & \\
\hline 6 & 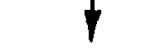 & 5 & 0 & \\
\hline 7 & $\dot{A}$ & 5 & 180 & \\
\hline 8 & B & .15 & 0 & \\
\hline 9 & 1 & 15 & 180 & \\
\hline 10 & & $80-95$ & 0 & \\
\hline 11 & & $80-95$ & 180 & \\
\hline 12 & $\phi$ & 50 & 0 & \\
\hline 13 & B & 50 & 90 & \\
\hline 14 & C & 20 & 0 & \\
\hline 15 & 1 & 20 & 180 & \\
\hline 16 & & $80-95$ & 0 & \\
\hline 17 & 1 & $80-9.5$ & 180 & \\
\hline 18 & $\dagger$ & 50 & 90 & $\dagger$ \\
\hline 19 & C & $80-95$ & 90 & 0 \\
\hline 20 & D & 15 & 0 & $5.88 \times 10^{-3}$ \\
\hline 21 & 1 & 15 & 0 & $8.81 \times 10^{-3}$ \\
\hline 22 & & 15 & 0 & $17.65 \times 10^{-3}$ \\
\hline 23 & & 85 & 90 & $5.88 \times 10^{-3}$ \\
\hline 24 & 1 & 85 & 90 & $8.81 \times 10^{-3}$ \\
\hline 25 & D & 85 & 90 & $17.65 \times 10^{-3}$ \\
\hline 26 & $\mathrm{E}$ & 17 & 0 & 0 \\
\hline 27 & 1 & 17 & 180 & \\
\hline 28 & & 95 & 0 & \\
\hline 29 & & 95 & 180 & \\
\hline 30 & 1 & 50 & 90 & \\
\hline 31 & $\mathrm{E}$ & 95 & 90 & \\
\hline 32 & $F$ & 10 & 0 & \\
\hline 33 & F & 20 & 0 & $\dagger$ \\
\hline 34 & $\mathrm{~F}$ & 95 & 180 & 0 \\
\hline
\end{tabular}

IV-2 

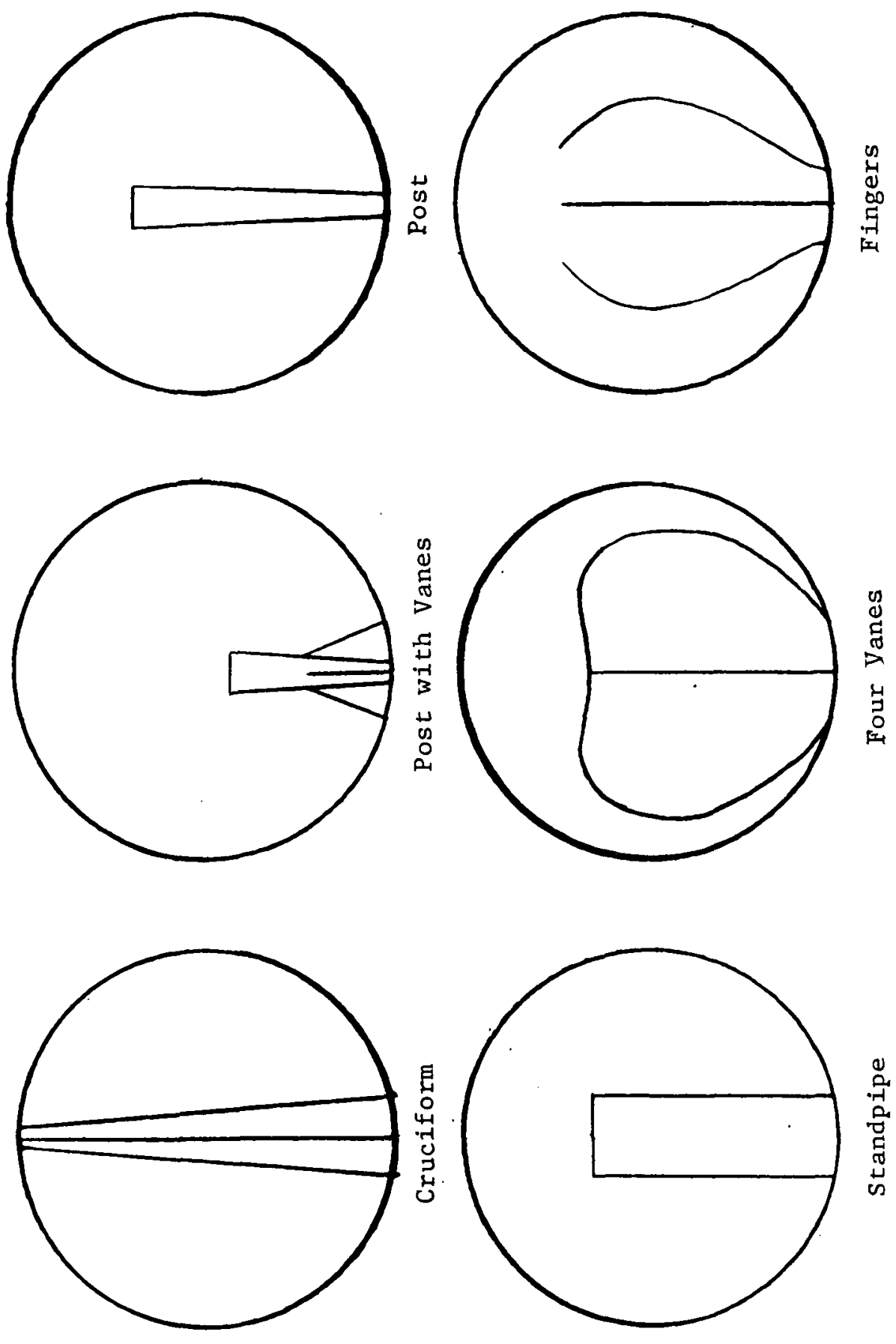

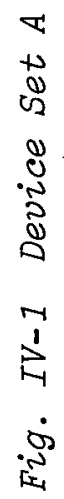



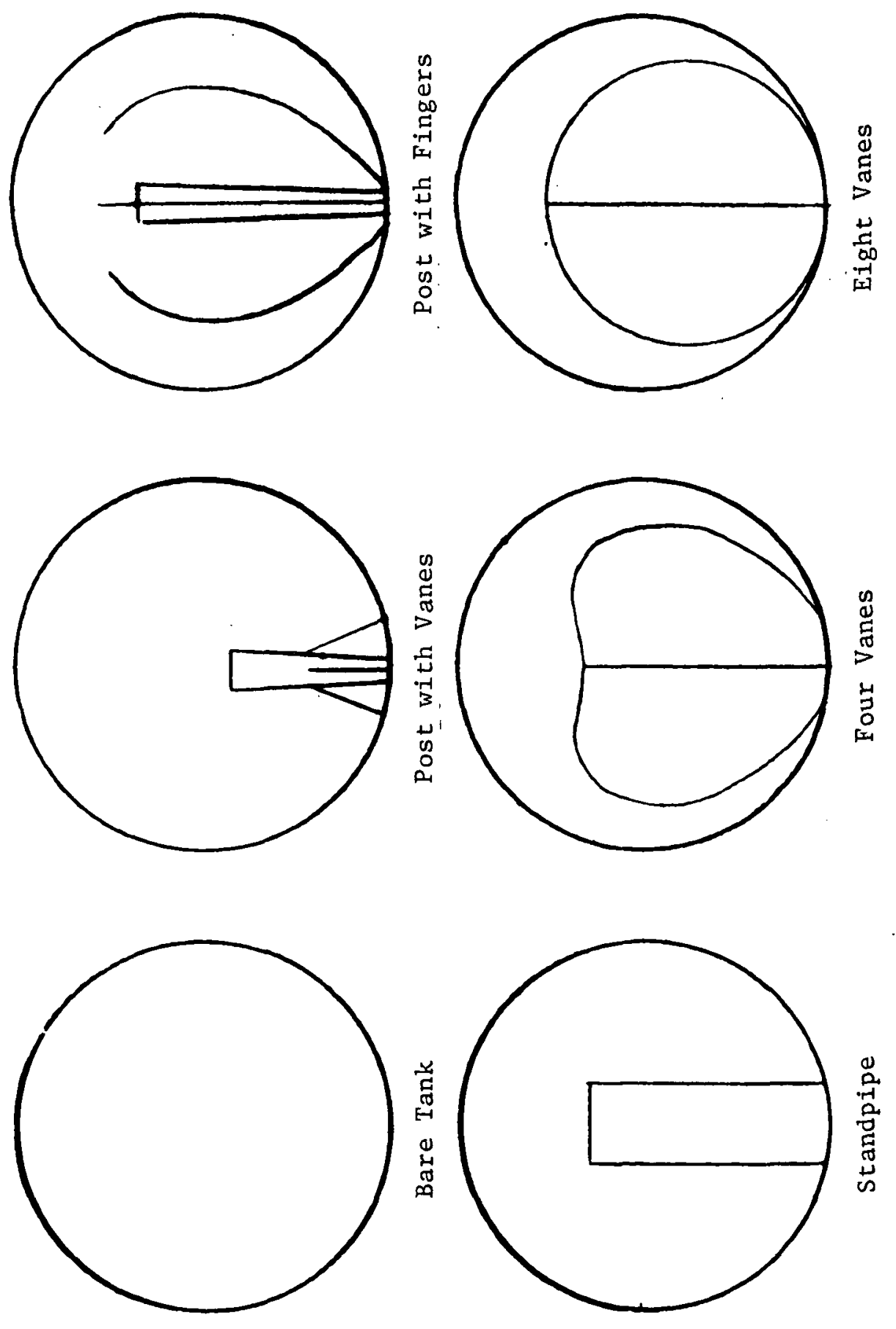

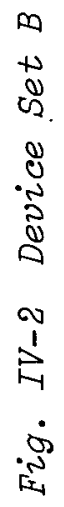

IV-4 

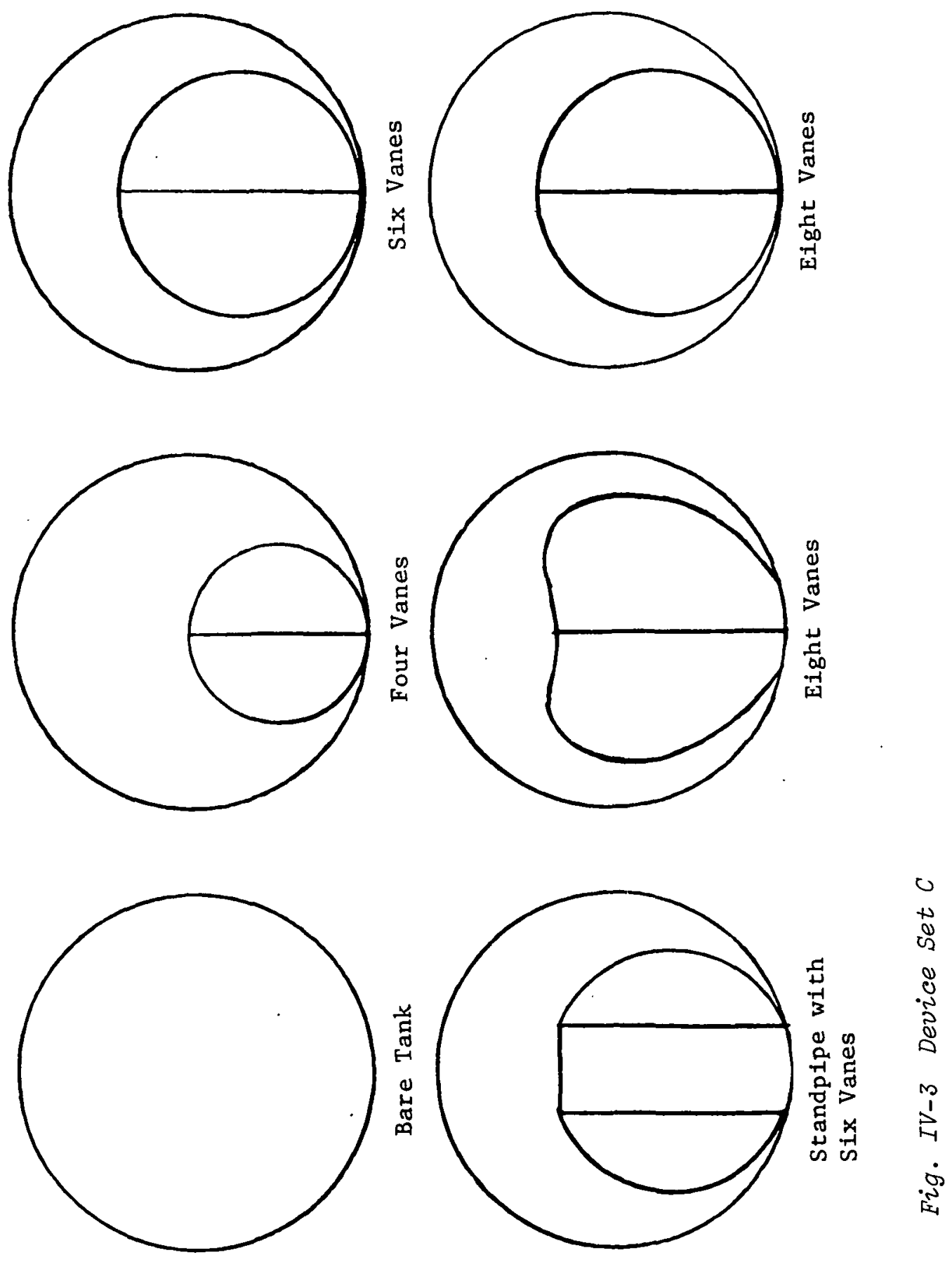

IV-5 

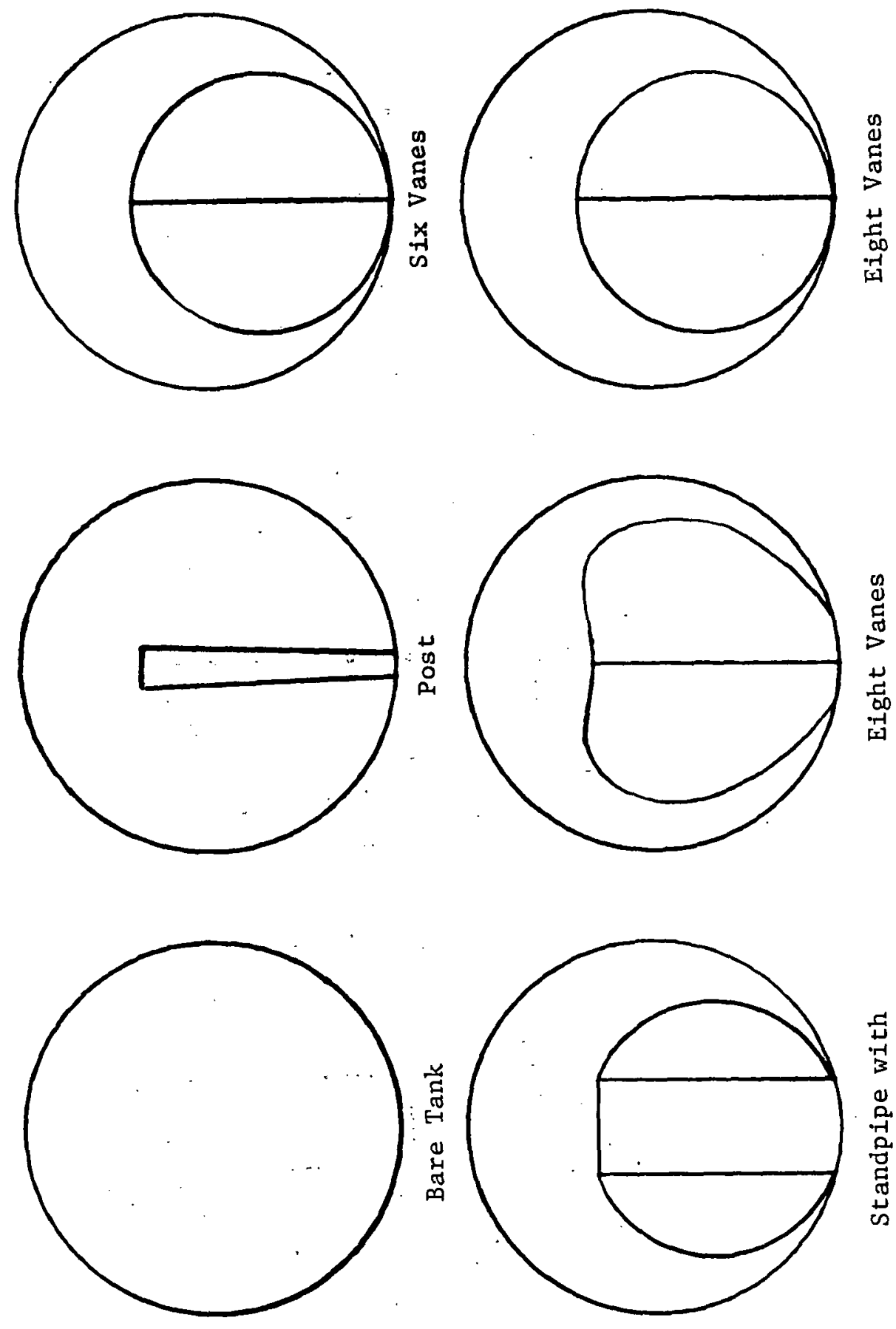

焉

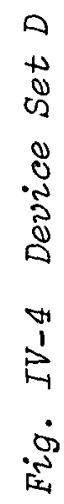



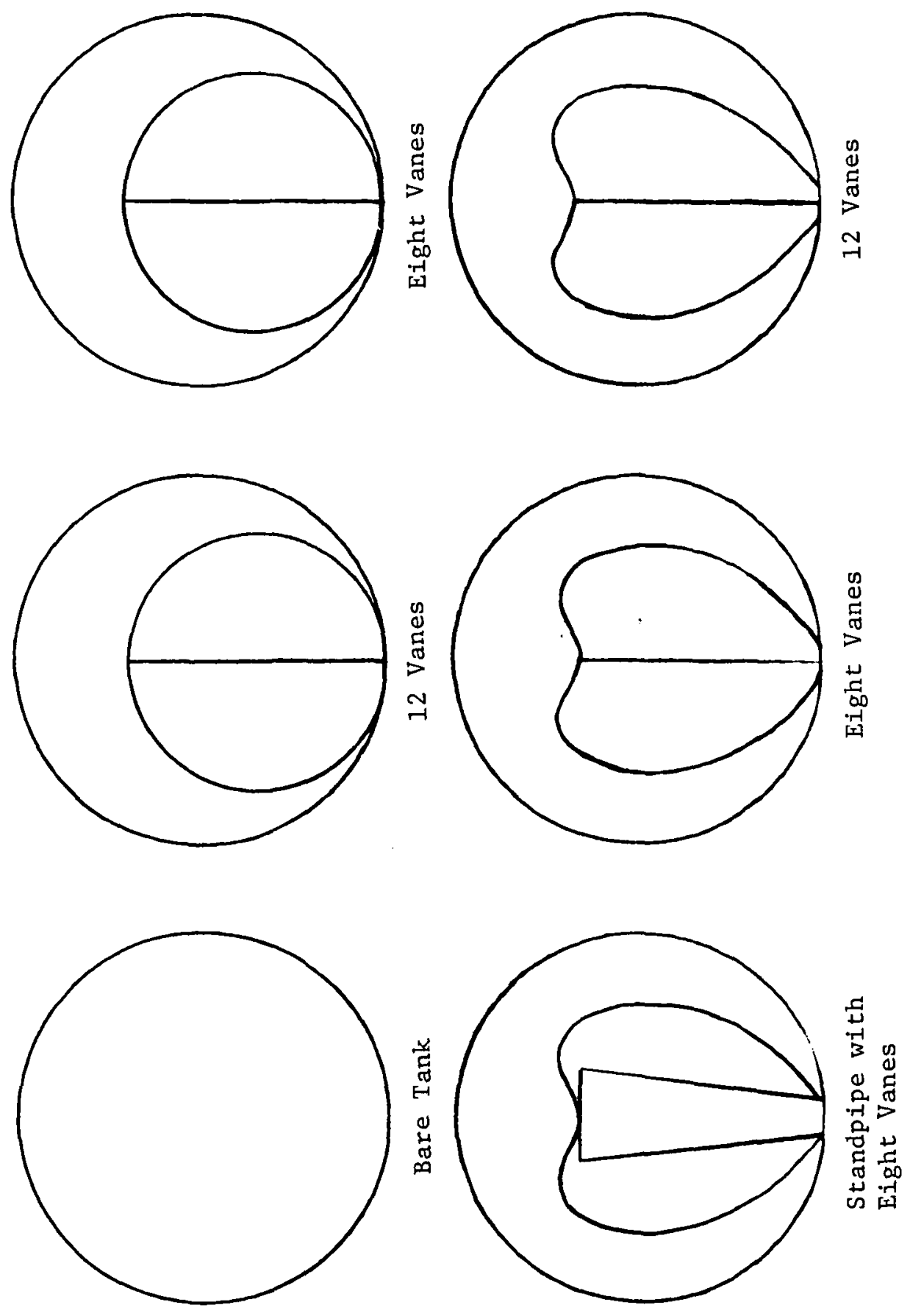

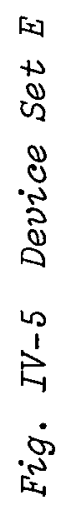



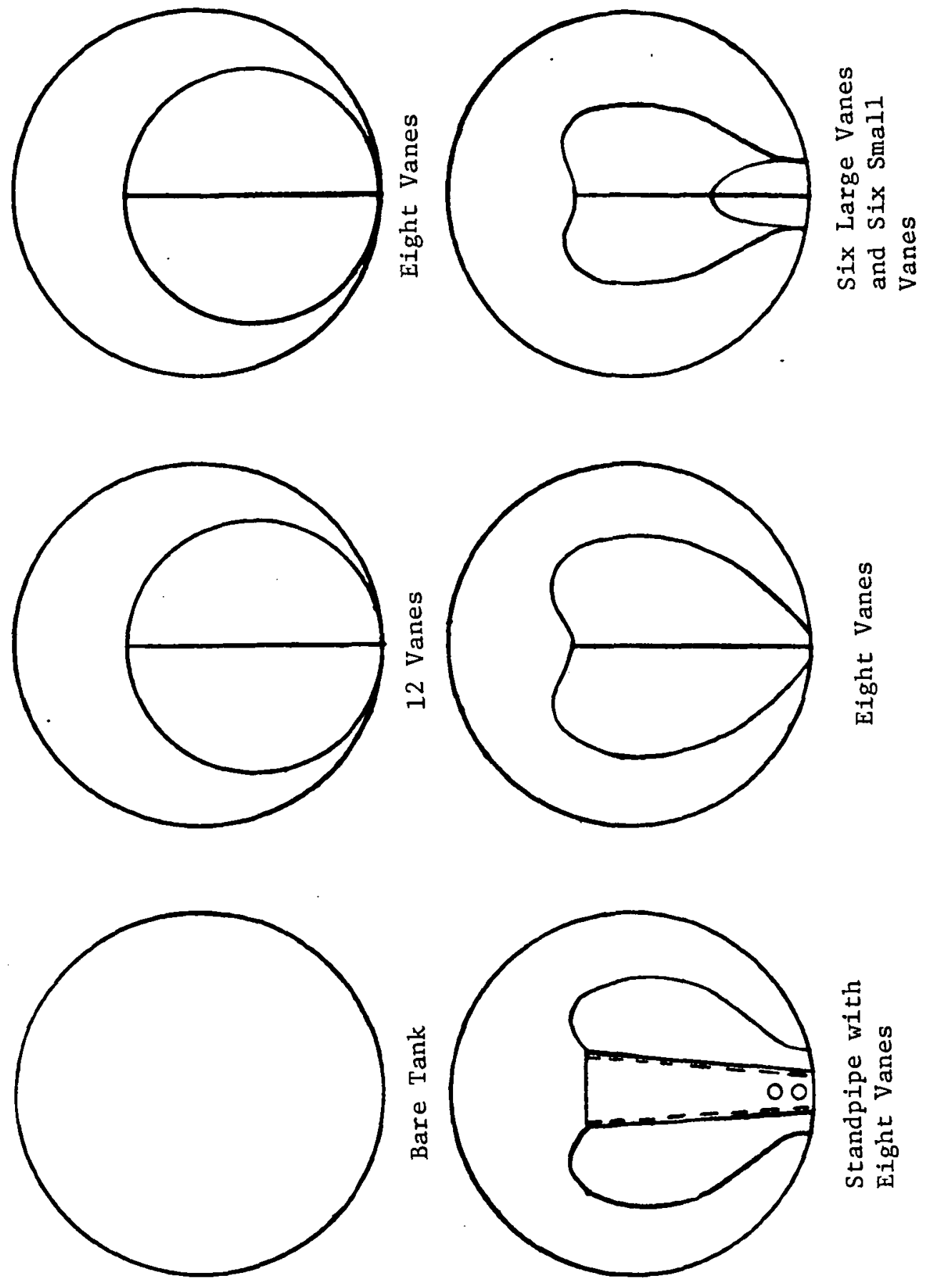

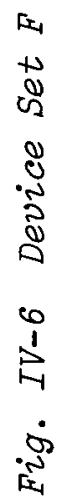


B. TEST APPARATUS

The test fixture used in the drop tests is shown in Figure IV-7 and IV-8. To facilitate the direct comparison of propellant acquisition candidates, the fixture was designed so that up to six scaled devices could be tested simultaneously. Each scaled device was contained within a spherical test container (small-scale propellant tank) formed by the metal test fixture sandwiched between two spherical glass segments. A thin rubber gasket provided a seal between the glass segments and the metal fixture. The glass segments were held and supported by a rubber 0 -ring and metal retainer ring combination.

The inside diameter of each scaled propellant tank was approximately 2 in. This size was selected to provide rapid reorientation of the liquid-gas interface from the flat one-g configuration to the low-g configuration (minimize transition time and maximize low-g time). The selection of glass for the test containers was based on a material survey which included glass, plexiglass, and polycarbonate. Based on chemical resistance to methanol, cost, and availability, glass was superior. Finally, methanol was selected as the test 1 iquid because it was readily available, easy to work with, and has a reasonably high kinematic surface tension which is desirable for reducing reorientation time.

The devices were fabricated from metal and plastic. Clear sheet Lexan was used to make the vane-type devices because it allowed an unrestricted view of the interface about the device. Some of the devices fabricated are shown in Figure IV-9. 


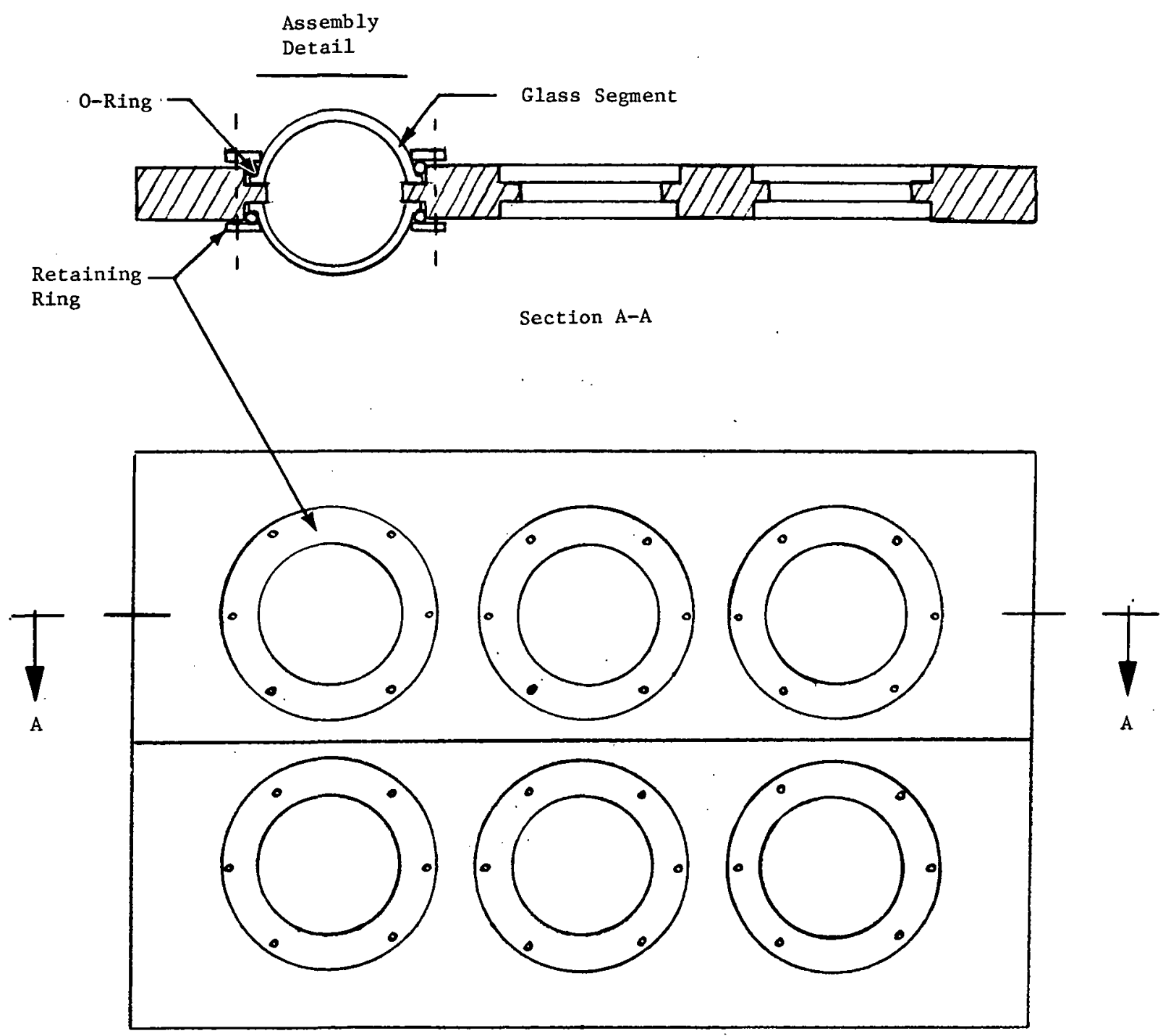

Fig. IV-7 Test Fixture

IV-10 


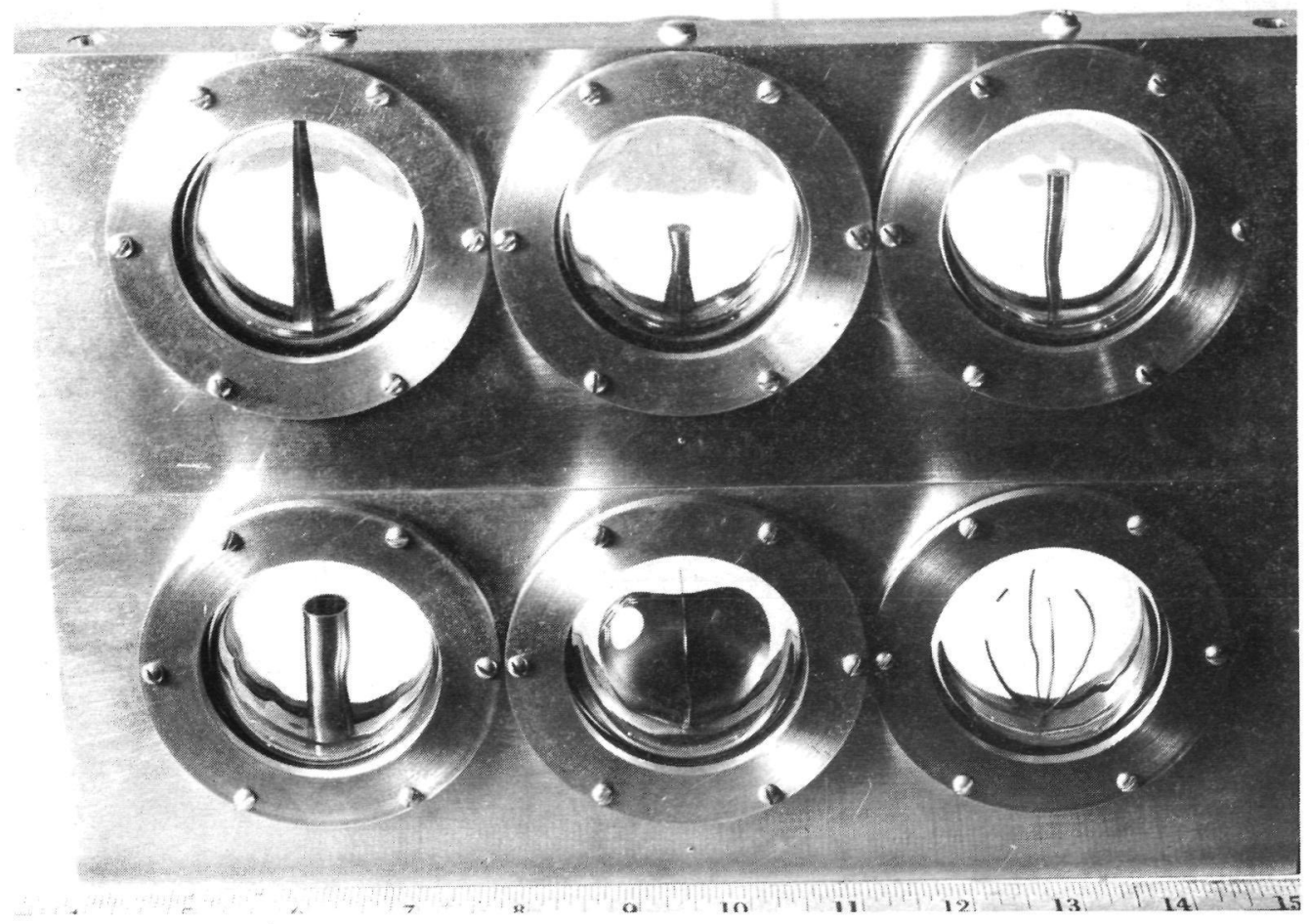

Fig. IV-8 Test Fixture with Devices Installed

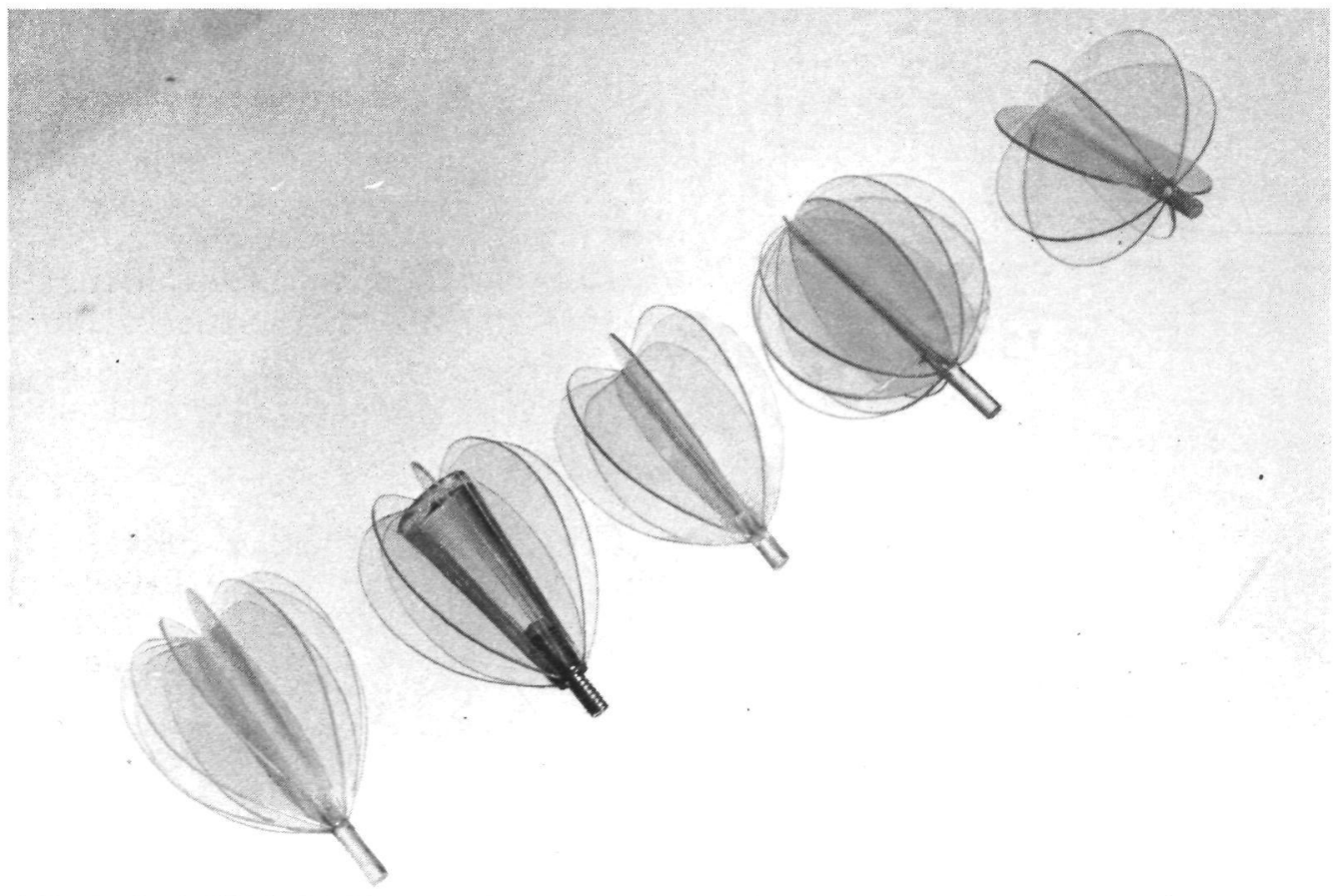

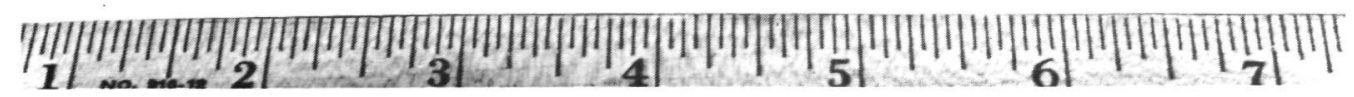

Fig. IV-9 Vane-Type Device Models 
C.

TEST RESULTS

A different aspect of the operation of the acquisition devices was tested with each of the three fixture orientations. At the $0^{\circ}$ orientation, the transition from high to low Bond number represents the termination of an engine burn. For low ullage volumes, positioning of the bubble during the test is of interest. The orientation of the liquid about the device is demonstrated at the lower liquid volumes.

The $90^{\circ}$ orientation represents the reorientation of the liquid following a strong lateral disturbance. At $180^{\circ}$ orientation, the worst-case conditions for the reorientation of the liquid are imposed. At low liquid levels, the operation of the communication channels can be observed with this orientation.

The results of the tests performed on each set of devices are shown in Fig. IV-10 thru IV-15. In each case, the photograph shows the liquid orientation at the end of the 2.1-sec test period. By referring to Fig. IV-1 thru IV-6 the devices being tested can be determined; Table IV-1 provides the test conditions.

1. Zero-g Tests

a. Cruciform - A scale model of the candidate cruciform device was evaluated in tests 1 through 7. At low liquid volumes (tests 1 and 6 ), the device positions liquid at the outlet. Liquid can be seen filling the length of the device during the test. A static interface shape had not been reached by the end of the test. In test 7 , there was neither enough liquid nor test time to observe where the liquid was being positioned. At the end of the test, most of the liquid was in the communication channels. Test 6 demonstrated the positioning capability of the cruciform relative to the other devices in the set. Since the cruciform fills by pumping liquid away from its base, it held only a part of the liquid over the outlet.

In tests 2, 3, 4, and 5, the positioning of the liquid at small ullage volumes was observed. Initially, the bubble was toriodal as it was pushed away from the outlet. But in all cases, the bubble became spherical and was positioned off-axis to one side of the device. 

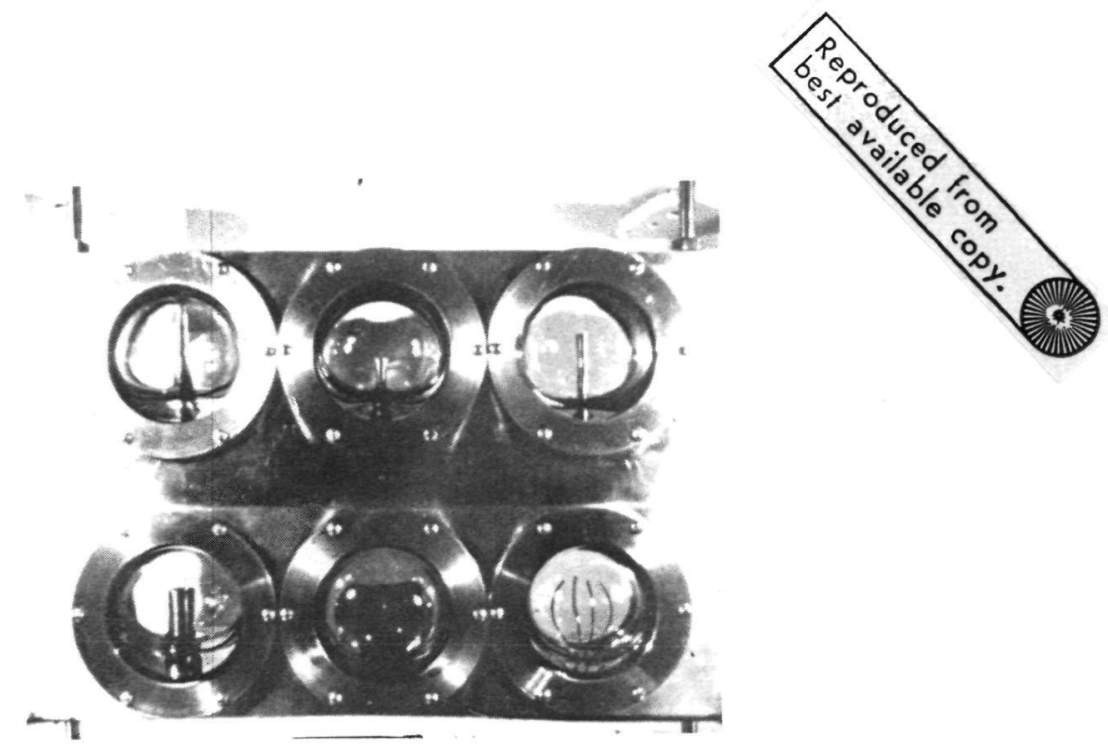

(a) Test 1

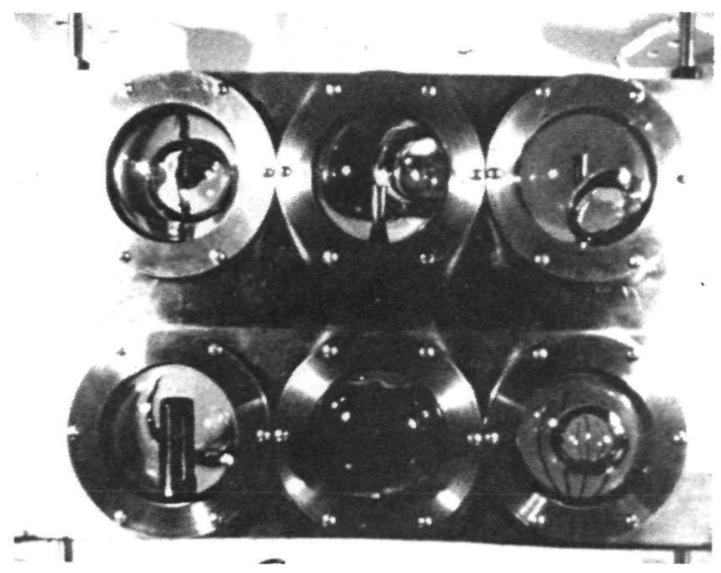

(b) Test 2

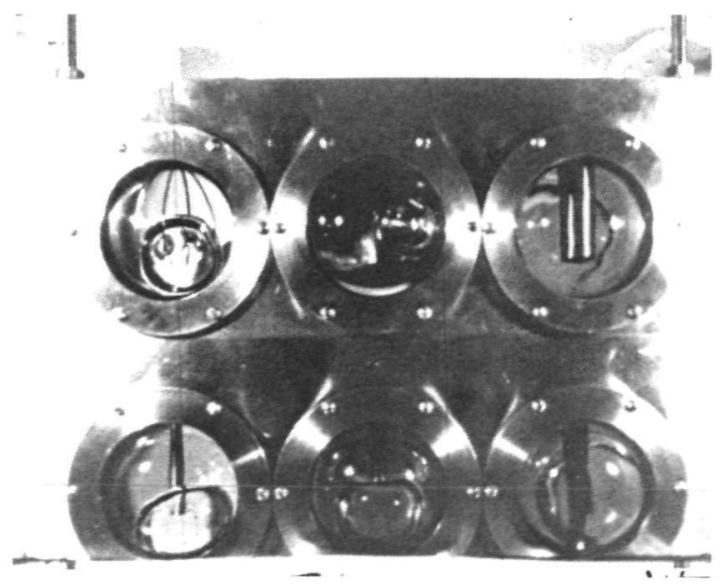

(c) Test 3

Fig. IV-10 Device Set A Tests 


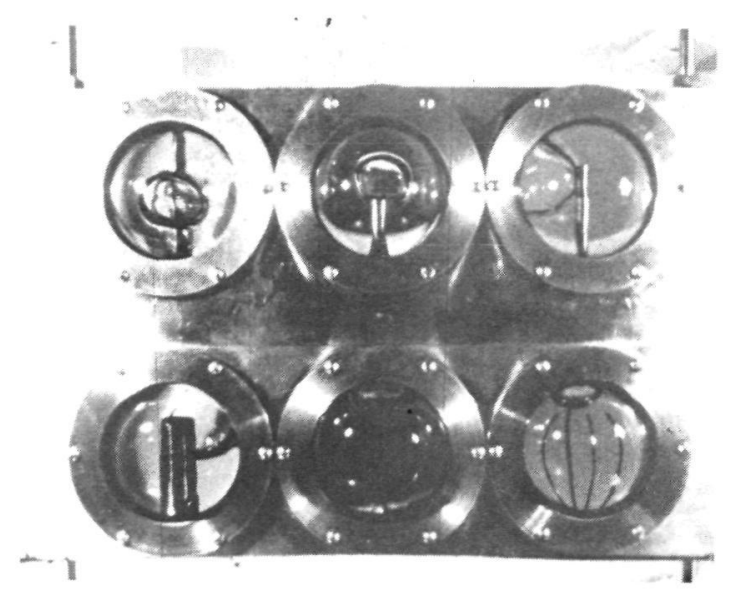

(d) Test 4

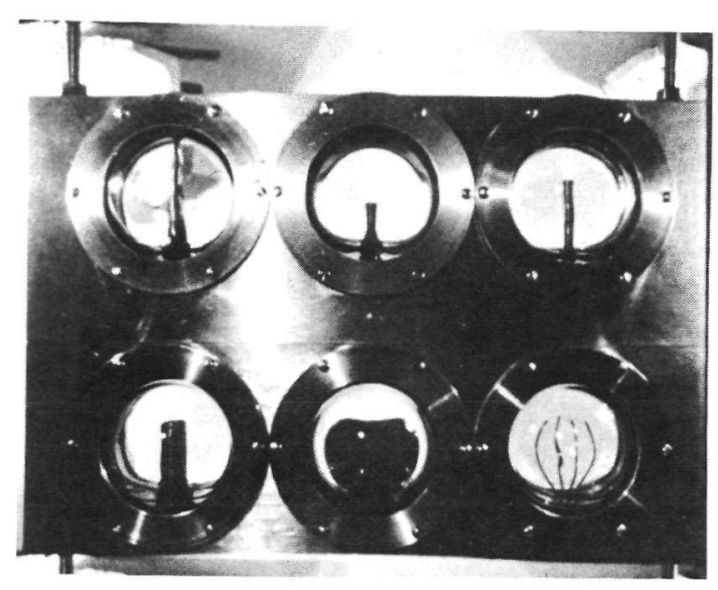

(f) Test 6

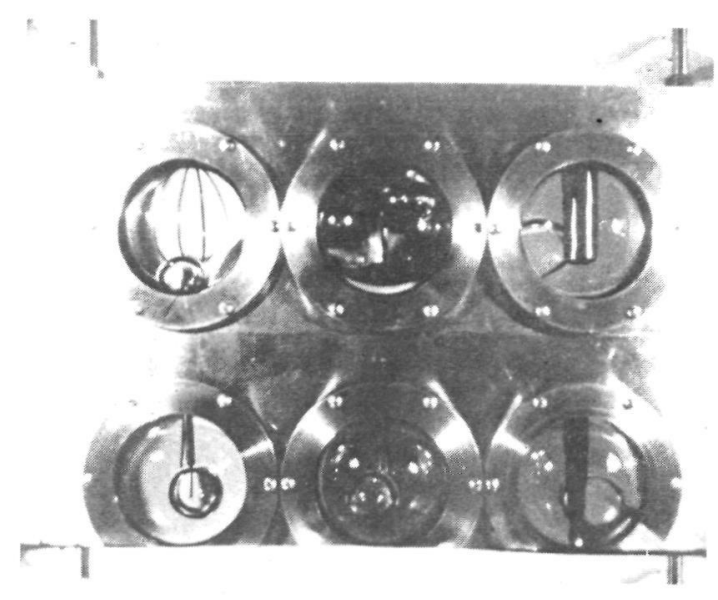

(e) Test 5

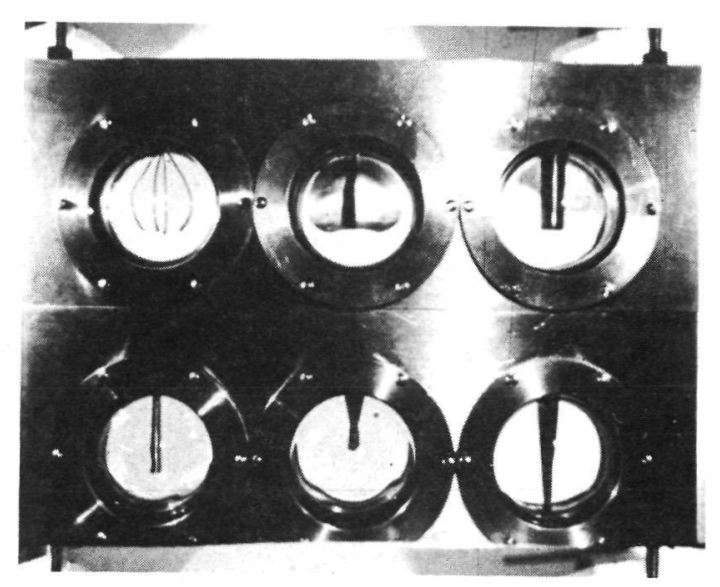

(g) Test 7

Fig. IV-10 (conel) 


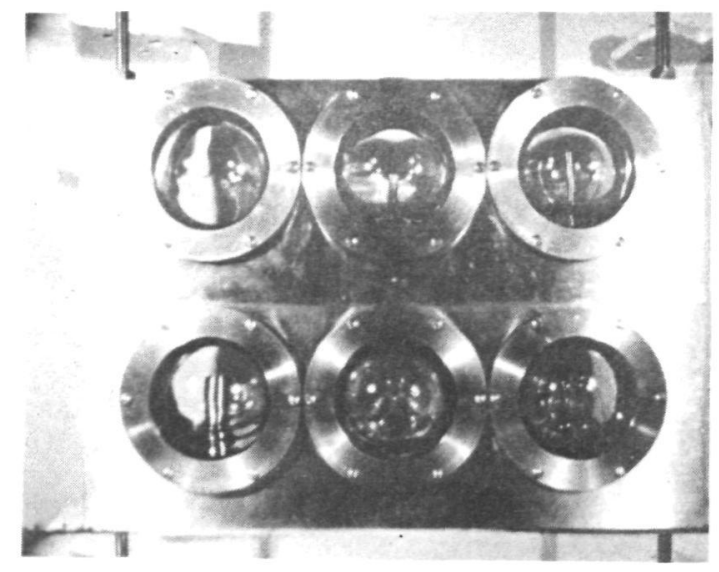

(a) Test 8

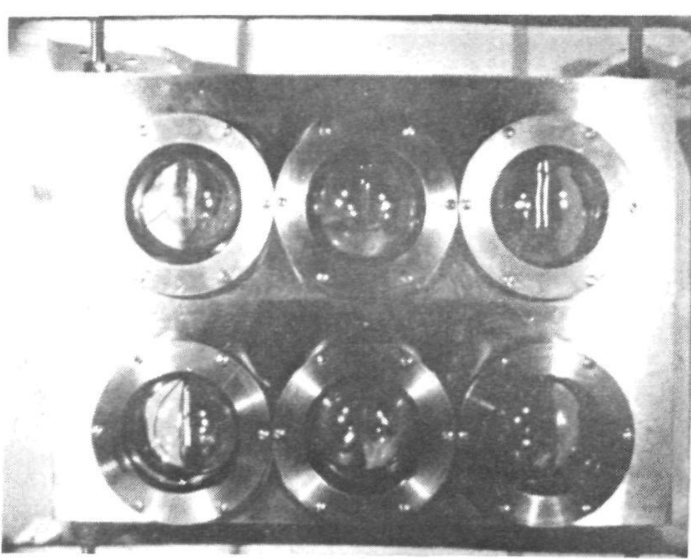

(b) Test 9

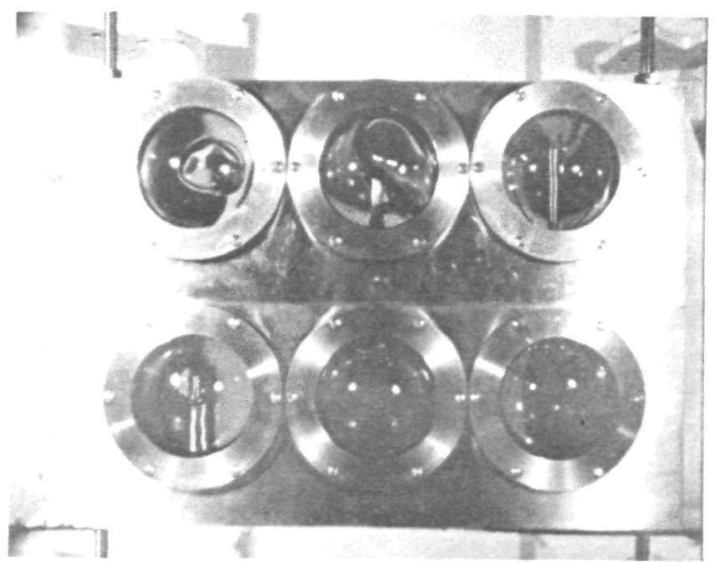

(c) Test 10

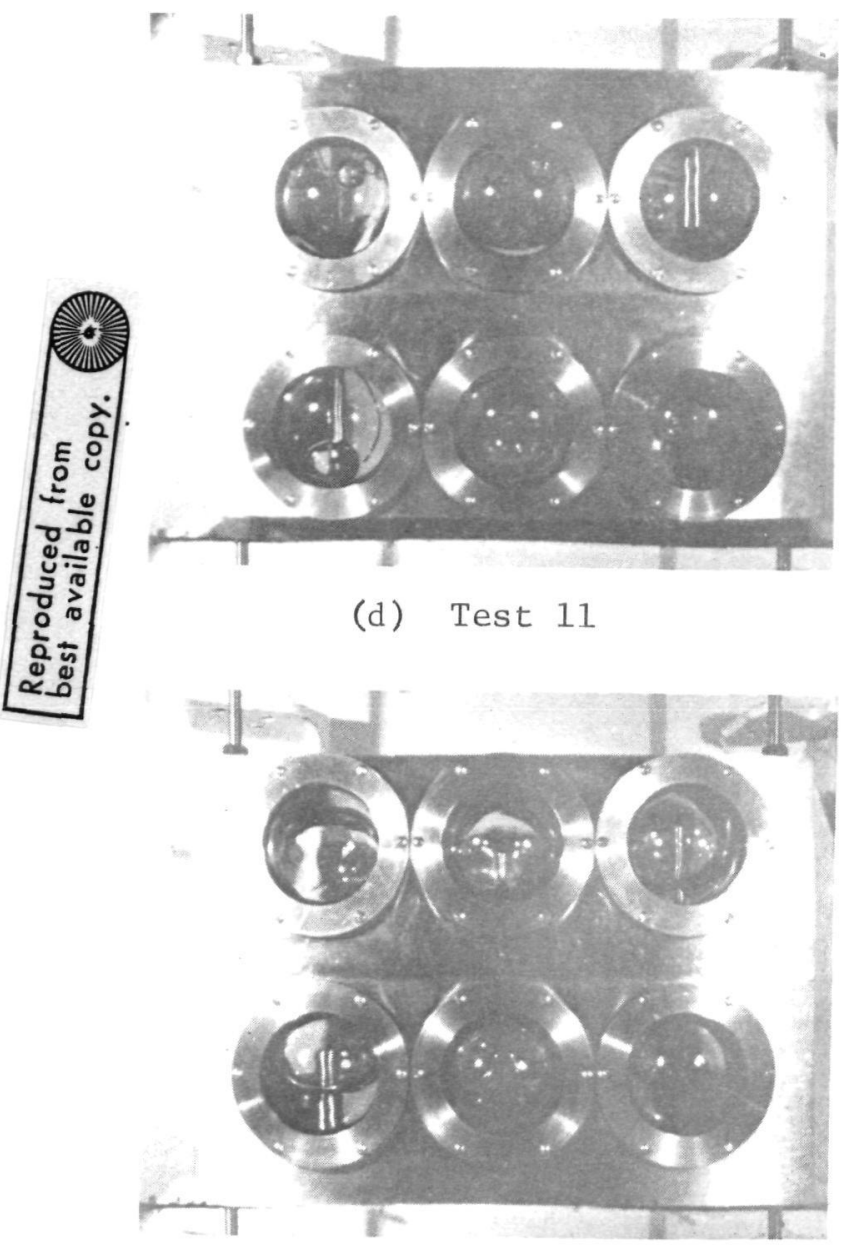

(e) Test 12

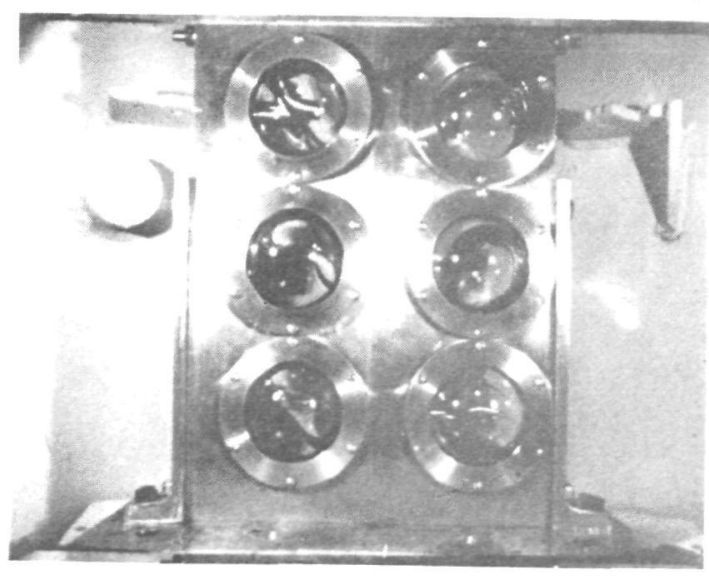

(f) Test 13

Fig. IV-11 Device Set B Tests 


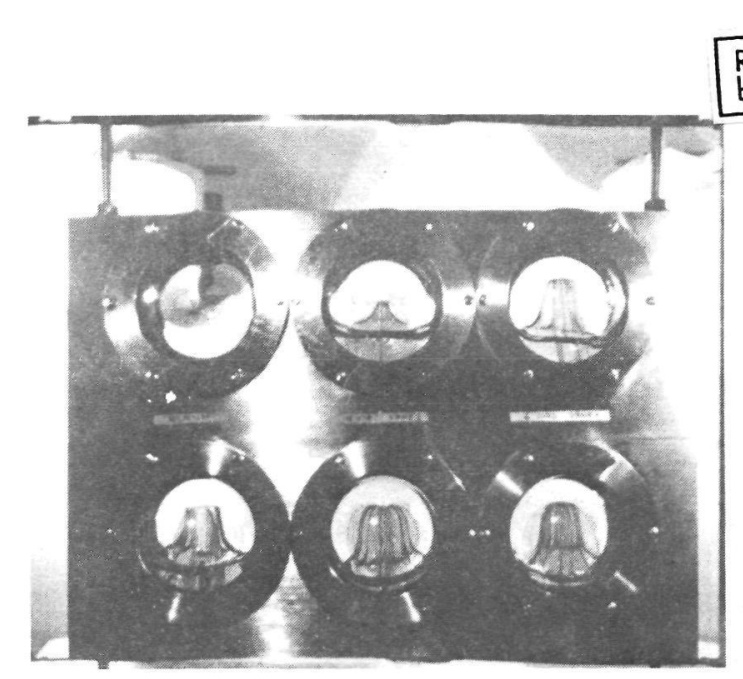

(a) Test 14

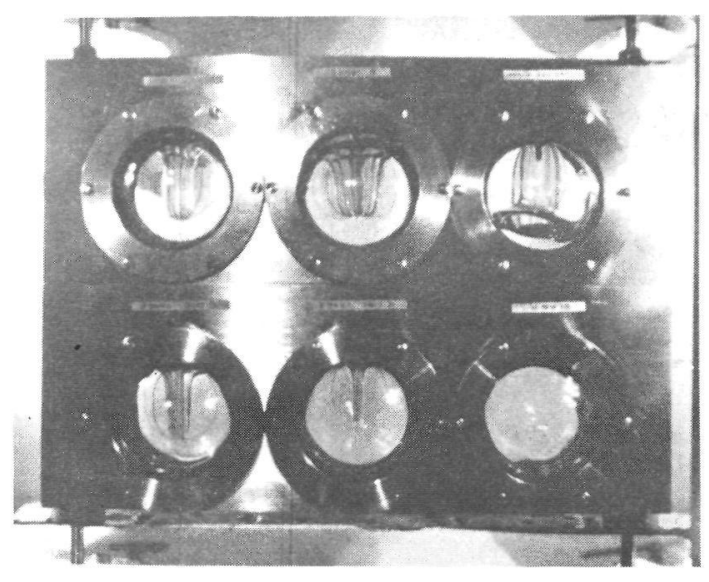

(b) Test 15

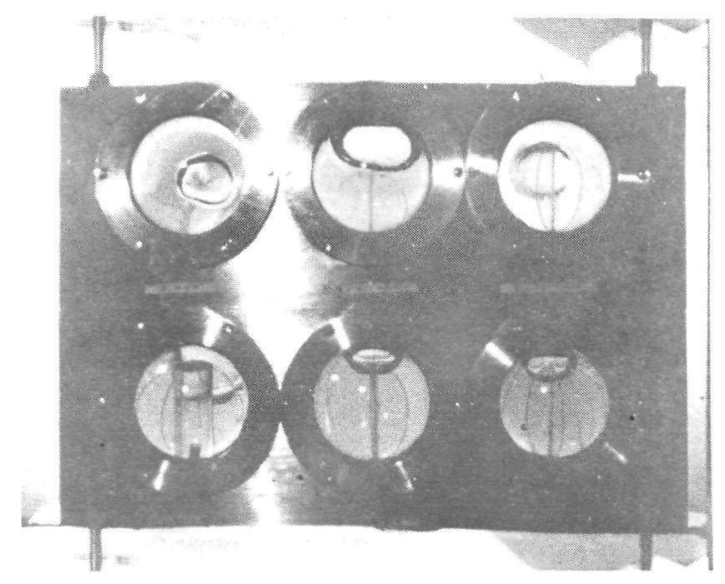

(c) Test 16
Reproduced from

best available copy.

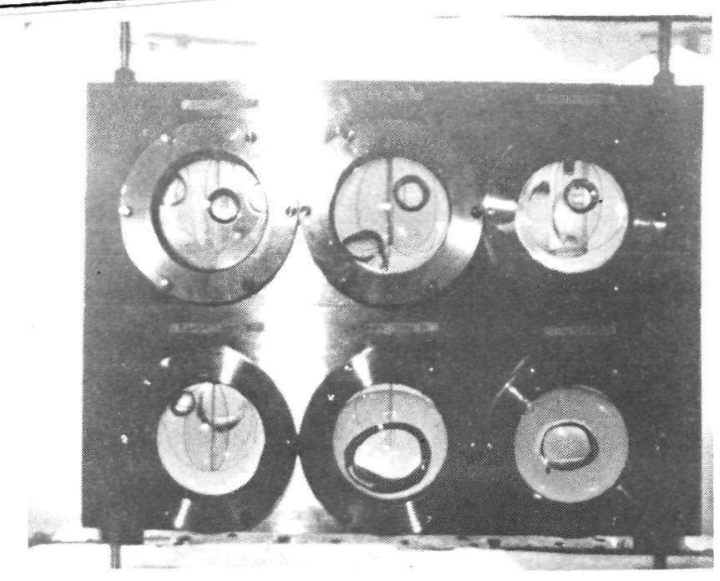

(d) Test 17

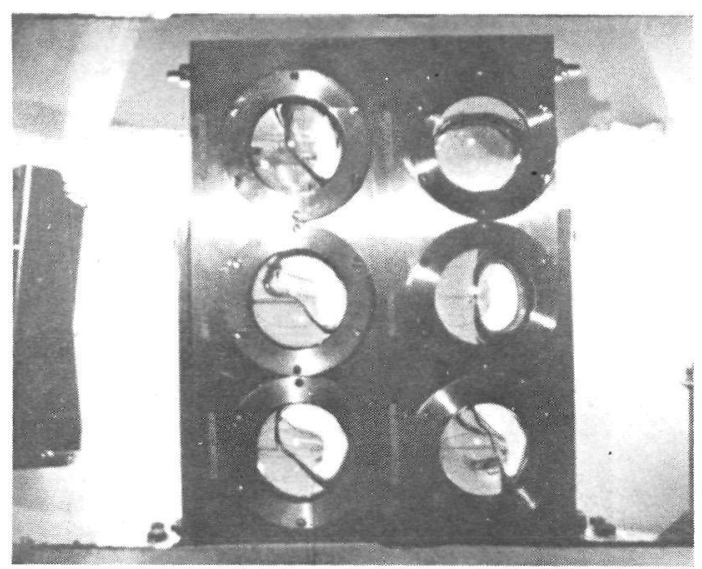

(e) Test 18

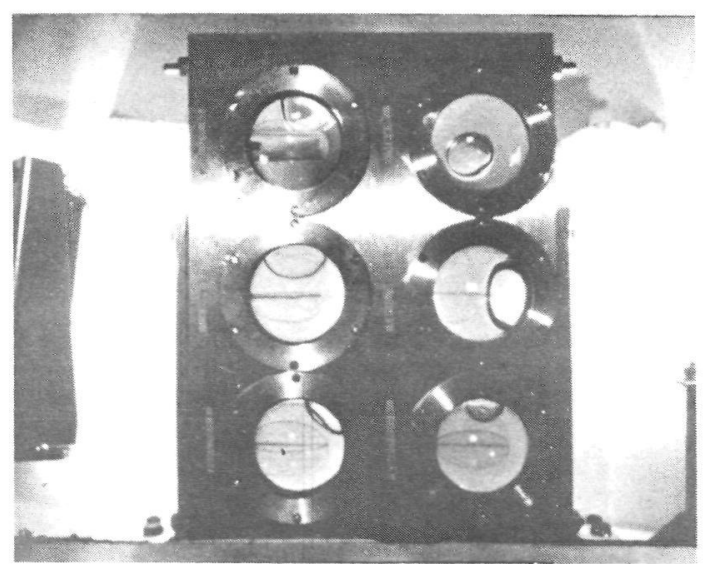

(f) $\quad$ Test 19

Fig. IV-12 Device Set C Tests 


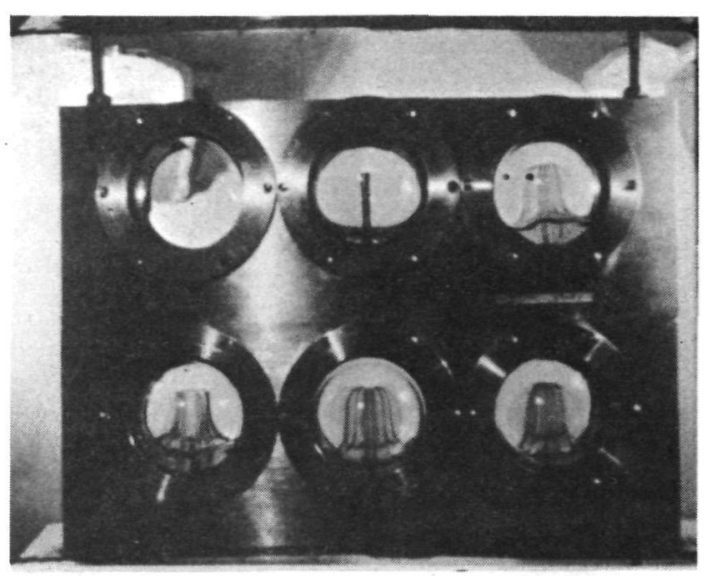

(a) Test 20

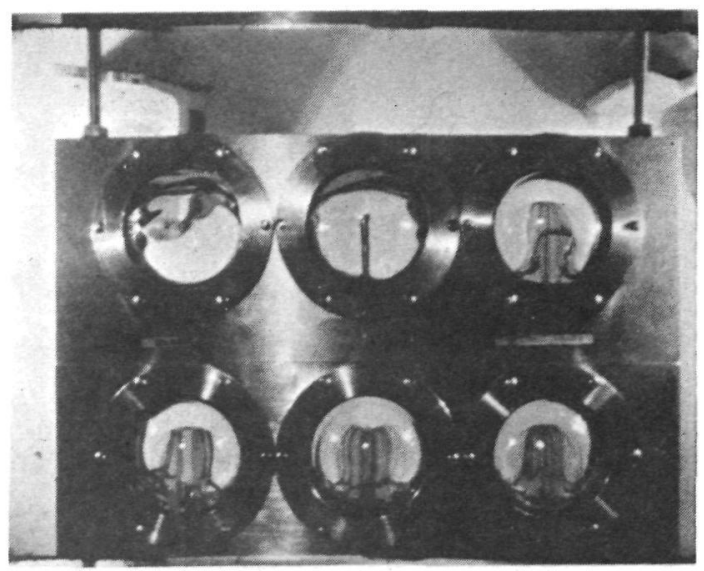

(b) Test 21

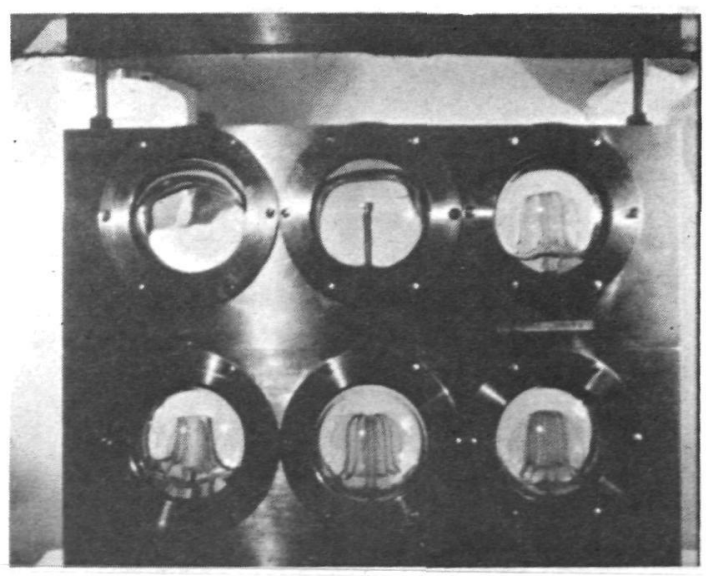

(c) Test 22

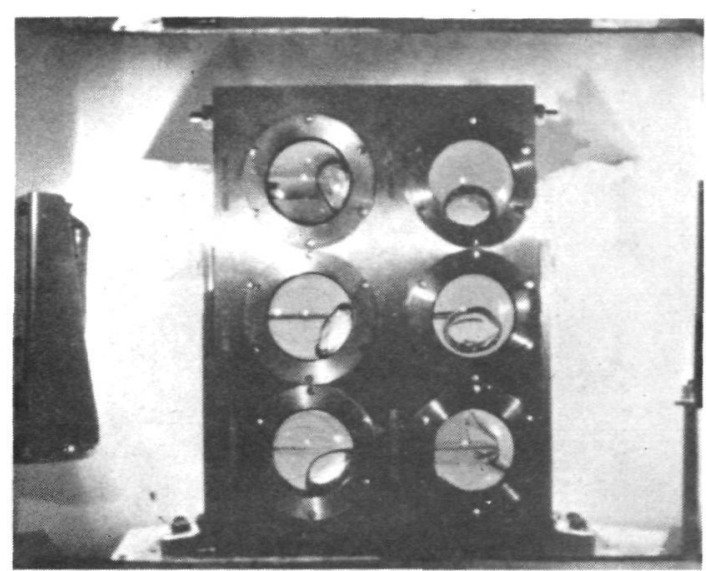

(d) Test 23

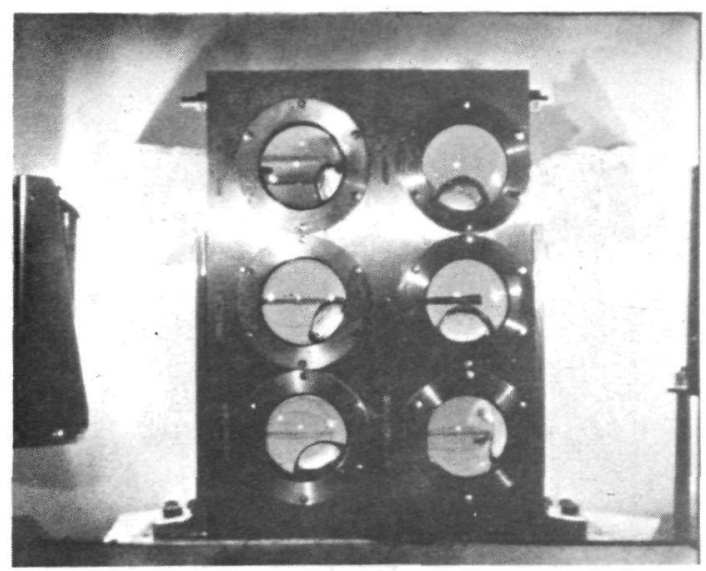

(e) Test 24

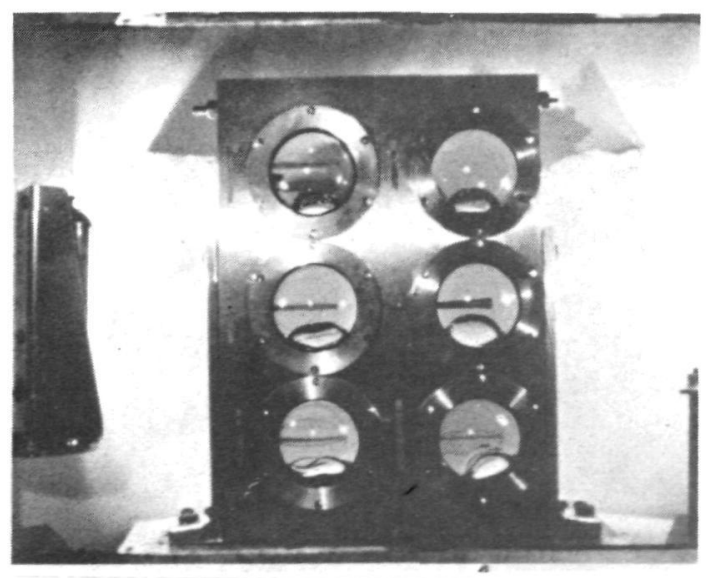

(f) Test 25

Fig. IV-13 Device Set D Tests 


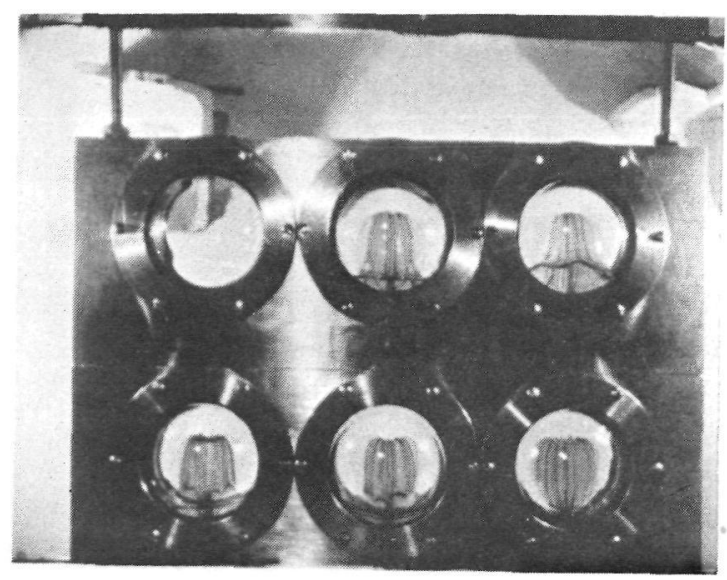

(a) Test 26

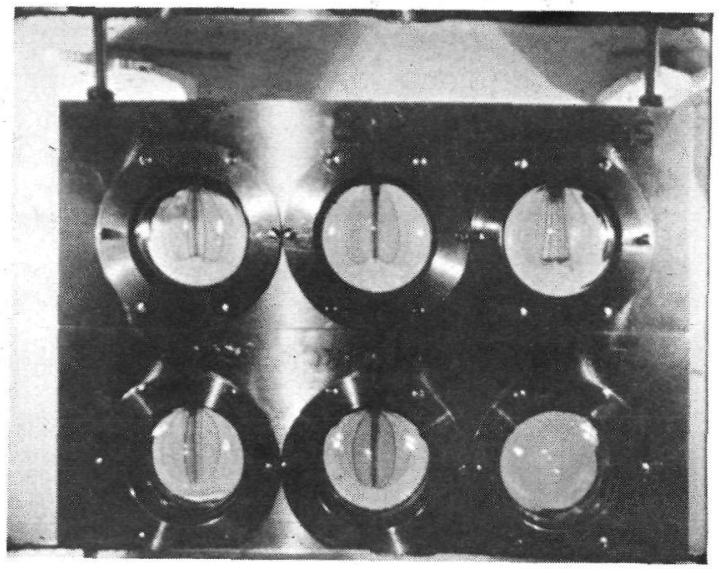

(b) Test 27

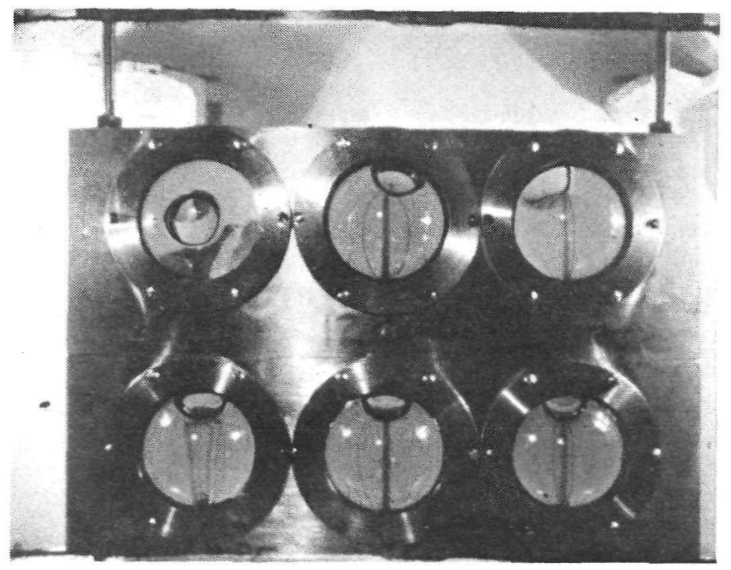

(c) Test 28

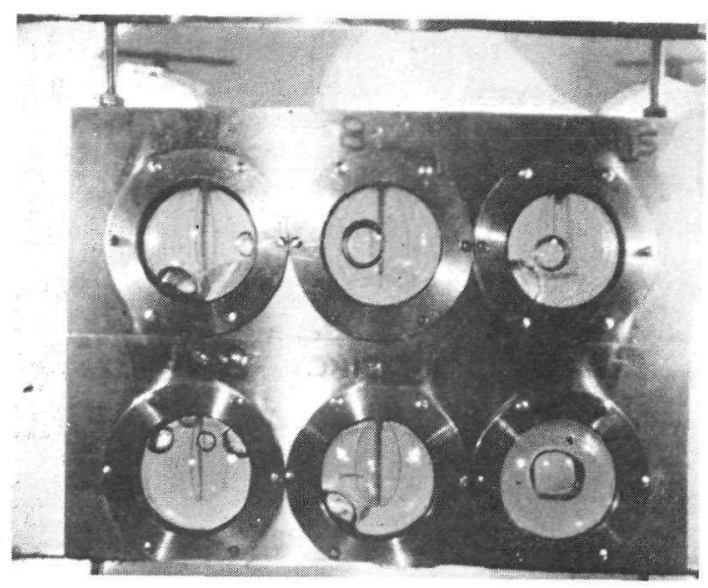

(d) Test 29

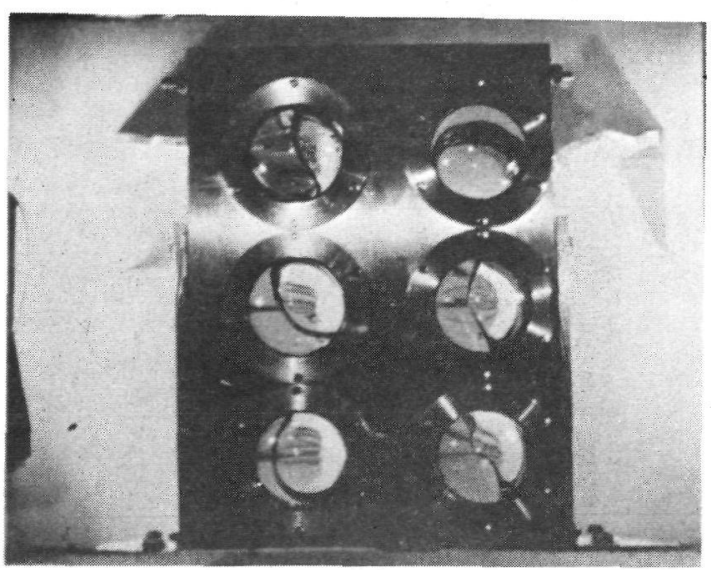

(e) Test 30

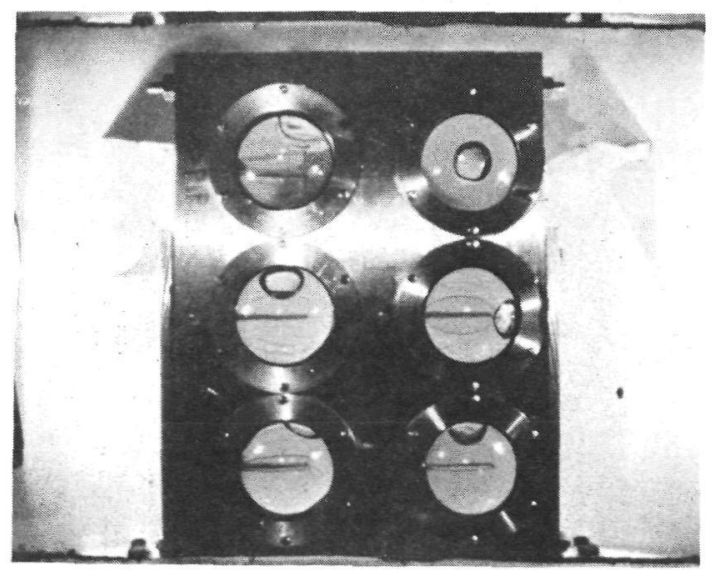

(f) Test 31

Fig. IV-14 Device Set E Tests 


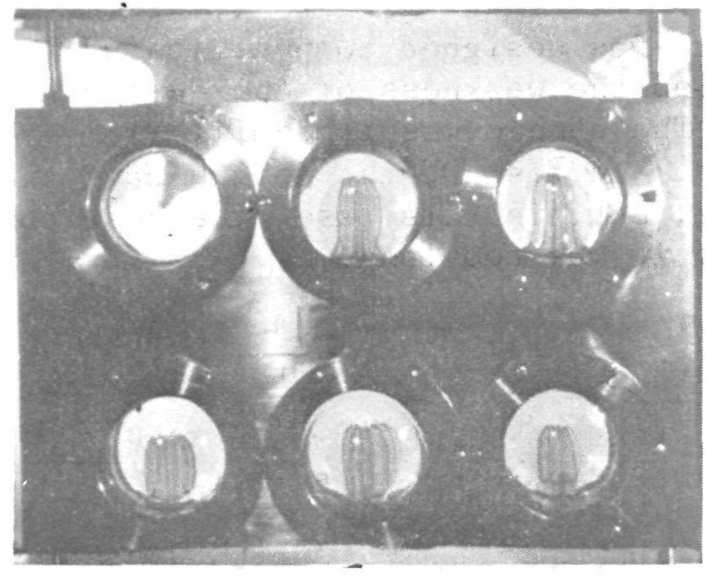

(a) Test 32
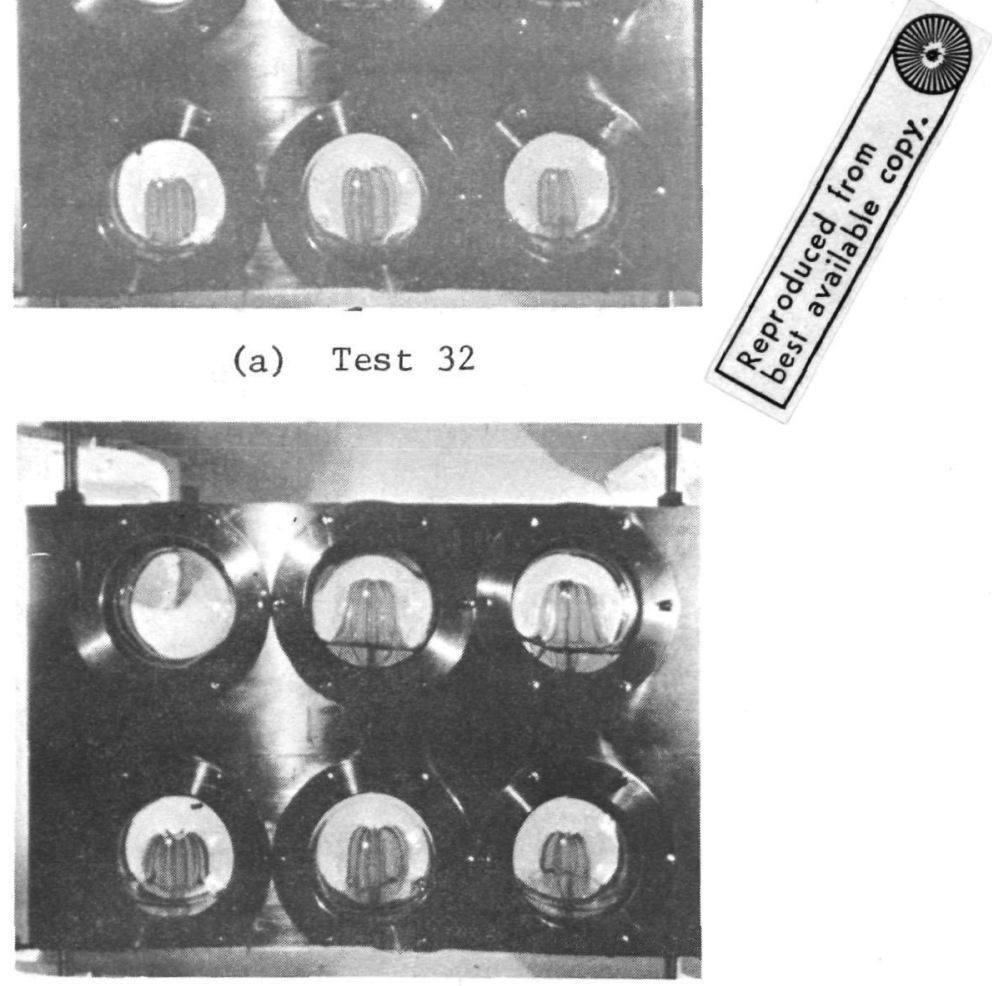

(b) Test 33

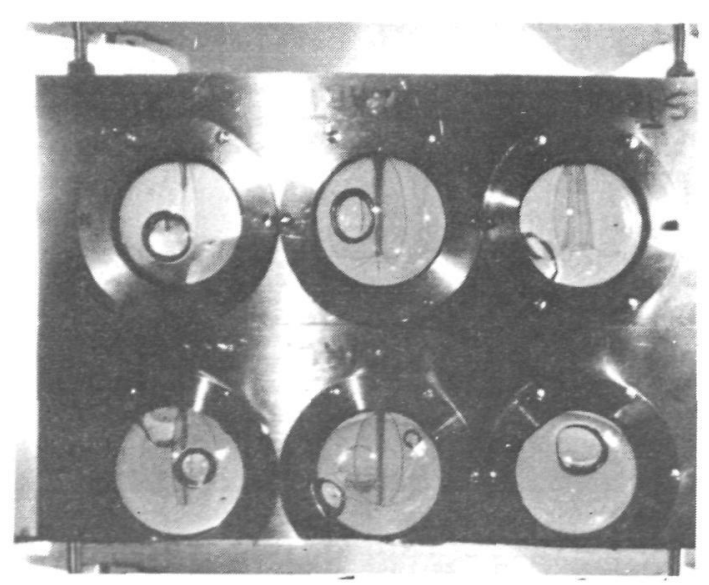

(c) Test 34

Fig. IV-15 Device Set F Test 
¿. Eost - The post device was designed to position a $5 \%$ ullage bubble. Test 5 illustrated the weakness of the design as discussed in the analysis. The bubble was in equilibrium, positioned to the side of the device. In test 3, the device came the closest to positioning the bubble, but, in this case, the post could not center the bubble because it was too long.

The post device with small vanes at the base was designed for a $20 \%$ ullage volume. In tests 4,5 , and 6 , it centered smaller ullage bubbles, but the bubbles had no stability. The slightest disturbance would position the bubble elsewhere. Test 2 produced a more typical result. In test 10, the device was still in the process of centering a $20 \%$ bubble when the test ended.

c. Standpipe - The standpipe tested had a $0.4 \mathrm{R}$ diameter and a height necessary to position a 5\% ullage bubble. It was hollow but did not have any openings at the base; at low liquid volumes there was no liquid inside the standpipe. At low iill levels, the device positioned most of the liquid over the outlet (see tests 1,6 , and 8 ). The other stable state, with the bubble offset to the side of the device, was demonstrated in the tests with ullage volumes of $85 \%$ and greater. The bubble was not centered in any of the tests. In tests 2,3 and 4, the bubble was behind the standpipe, as viewed by the camera.

d. Post with Fingers - In device set A, the fingers were tested by themselves. Test 2 illustrated one of their disadvantages; the bubble became entrapped within the fingers. In Tests 3 and 5 , the device was capable of holding the bubble centered. The post was then added to the fingers to improve the positioning of the bubble. Centering of the bubble occurred in tests 10 and 11 .

The motion of the ullage bubble during the test is of interest in the case of some of the devices. As the bubble formed at high fill levels, a downward force was applied to the bubble. For example, with the fingers in test 4, the bubble formed, traveled to the bottom of the tank, rebounded, and returned to the top of the tank yielding the final position shown in the photograph. In test 2 , the fingers entrapped the bubble after it rebounded from the bottom of the tank. The initial force applied to the bubble probably accounts for the bubble moving to the position on the top of the fingers in test 3 . 
e. Vones - A number of vane devices with various profiles and numbers of vanes were tested. Operation of the devices at lowand high-fill levels, and the vane pumping capability are discussed.

1) Low-Fill Levels - The number of vanes has a significant effect on the amount of liquid that is positioned directly over the outlet. Test 14 offers a good relative comparison. The devices with eight vanes pulled liquid up within the vanes while the devices with four and six vanes held most of the liquid over the outlet. The difference between and eight-vane and 12-vane device is not as significant, as can be seen in tests 32 and 33 . The vane profile near the outlet will influence the amount of liquid held over the outlet. An example is shown by the results from test 26. Due to the heart-shaped profile of the 12-vane device, all of the liquid was pumped into and held within the vanes. In the same test, however, an eight-vane device with the same profile allowed some liquid to be over the outlet. In comparison, the circular profile vanes, which are closer to the tank wall, allowed more liquid to be over the outlet. Similar results were obtained in tests 32 and 33 with the device composed of large and small vanes.

2) High Fill Levels - With a $0^{\circ}$ orientation and small ullage volumes, the bubble should form and remain centered. Again, the profile and number of vanes influenced device performance. In test 16, the bubble was displaced to the side of the device with six circular vanes, while the device with eight circular vanes exhibits better ullage bubble centering. The eight-vane device with the heart-shaped profile centered the bubble best in that test. The same result was obtained in test 28 ; both of the devices with circular vanes had offset bubbles, while the heartshaped vane devices centered the bubble.

3) Pumping Capability - The 90 and $180^{\circ}$ orientation tests were conducted to demonstrate device pumping capability. Given sufficient time, the devices should be able to center the ullàge bubble from its initially displaced position. In the $90^{\circ}$ orientation tests with $50 \%$ fill, most of the vane devices were still in the process of orienting the bubble at the end of the test. The sharp curvature of the interfaces in tests 18 and 30 showed that equilibrium had not been established. In test 18, the four-vane device centered the bubble before the end of the test. 
The existence of other stable locations for the bubble was demonstrated when the devices were tested with small ullage volumes and $90^{\circ}$ orientation.

In tests 19 and 31 , the bubbles remained in an equilibrium position to the side of the devices, except for one device in each test. In test 19 , the small device with four circular vanes did center the bubble. The same was true of the 12-circular-vaned device in test 31 . The reason that this other stable position exists is discussed in the Chapter III analysis.

Breakup of the ullage bubble occurred with some of the vane profiles when they were tested at an orientation of $180^{\circ}$. The effect of the breakup can be seen in tests 17, 29, and 34. Devices were designed, based on the analysis, to eliminate the breakup. The six- and eight-vane devices with a heart-shaped profile, employed in test 34 , showed that breakup will not occur if that profile is used.

f. Standpipe with Vones - Device sets C, E, and F each had a standpipe with vanes and a vane device having the same vane profile and number vanes. This allowed a comparison of the operation of a vane device with and without a standpipe. The standpipe in set $F$ is hollow and has openings near the base, while the other standpipes were solid posts.

In test 14, the standpipe with vanes held more liquid closely about the outlet than the device with vanes alone. The same was true for these two devices in test 33. Centering of the ullage bubble was still a problem with the circular-vaned standpipe, as shown by test 16 . Centering occurred with the standpipe having heart-shaped vanes (test 28). Pumping of the bubble was not improved by the standpipe and other stable locations for the bubble can exist (test 31). Bubble breakup was still a problem with the vaned standpipe (tests 17 and 29), but can be eliminated with the properly designed vane profile (test 34 ).

2. Communication Channels

The crevices formed at the joints of the glass spherical segments and the flange of the test fixture served as communication channels. Tests were run at low fill levels and a $180^{\circ}$ orientation to demonstrate the operation of the channels. The channels were quickly filled with liquid, and, toward the end of the test, liquid was observed filling the base of some of the devices. This was true in test 27 . In test 15, the liquid was initially in contact with the top of the devices, so liquid was oriented both by pumping along the device itself and by pumping along the channels. A 
gap between the base of the standpipe with vanes and the flange of the fixture caused the liquid to remain oriented at the top of the tank. Once the communication channels filled that gap with liquid, orientation of the liquid would continue until all of the liquid was pumped to the device.

3. Stability Tests

a. Axial Stability - The first three stability tests were aimed at establishing the relative axial stability of the five devices in set $D$. A post device was included in this set to serve as a reference, since the axial stability of this device has been analyzed in detail.

Bond number was used as the scaling parameter to determine the spring force that would give the test fixture the required acceleration. The effect of inertial forces with respect to capillary forces is the same in both the model and the full-scale tank when the Bond numbers are identical. Selection of the three Bond numbers to be used was based on the stability curve for the post device (Fig. III-10) and available spring motors. Table IV-2 lists the forces and accelerations applied to the fixture in each test:

Table IV-2 Bond Numbers for Stability Tests

\begin{tabular}{|l|l|c|l|}
\hline $\begin{array}{l}\text { Test } \\
\text { No. }\end{array}$ & $\begin{array}{l}\text { Spring Motor } \\
\text { Force, } 1 b_{\mathrm{f}}\end{array}$ & Acceleration, g & $\begin{array}{l}\text { Bond } \\
\text { Number }\end{array}$ \\
\hline 20 & 2 & $5.88 \times 10^{-3}$ & 1.30 \\
21 & 3 & $8.82 \times 10^{-3}$ & 1.95 \\
22. & 6 & $17.70 \times 10^{-3}$ & 3.90 \\
\hline
\end{tabular}

Scaling factors, between the model and the full-size tanks are listed in Table IV-3.

For the fuel tanks, the accelerations modeled by the subscale tanks were on the order of $10^{-4} \mathrm{~g}$. For the oxidizer tanks, the testing simulated an actual acceleration of about $10^{-5} \mathrm{~g}$. These accelerations correspond to the adverse axial disturbances expected during the baseline and other typical missions. 
Table IV-3 Scaling Factors

\begin{tabular}{|c|c|c|}
\hline Propellant & $\begin{array}{l}\text { Tank Radius, } \\
\text { Actual } \\
\text { Model }\end{array}$ & $\begin{array}{l}\text { Acceleration } \\
\text { Model } \\
\text { Actual }\end{array}$ \\
\hline Flox & 17.7 & 965 \\
\hline $\mathrm{MMH}$ & 14.4 & 151 \\
\hline$F_{2}$ & 16.2 & 840 \\
\hline $\mathrm{N}_{2} \mathrm{H}_{4}$ & 16.2 & 113 \\
\hline
\end{tabular}

For each device and each Bond number there is a liquid volume that will remain oriented in a stable fashion at the bottom of the tank. When the acceleration acting on the tank is constant, liquid will continue to flow away from the device until this equilibrium volume is reached.

The post had a decreasing stable amount of liquid held about the device as the test acceleration was increased. These volumes corresponded to the volumes predicted by the analysis. In comparison, the vane devices have a much higher stability. Devices with eight vanes demonstrated more stability than devices with six vanes. The standpipe with vanes had the most stability of the five devices.

b. Lateral Stability - The results obtained from tests 23, 24, and 25 demonstrated the lateral stability of the five devices in set $D$. The same accelerations were used in this series of tests, so the scaling factors listed in Table IV-3 apply.

The post had very little lateral stability; the bubble was displaced to the side of the device.in all three tests. In test 25, the acceleration produced a hydrostatic head which exceeded the capillary force acting to center the bubble for all the devices. Comparing the relative displacement of the bubble in tests 23 and 24 , the relative pumping capability of the devices was established. It was found that the standpipe with vanes had the highest lateral stability of all the devices. 
V. COMPARATIVE EVALUATION

Two comparative evaluations were performed during the program. The first evaluation was devoted to comparing and rating the candidate propellant acquisition systems to select a system for recommendation to the Jet Propulsion Laboratory for further study and design. The second evaluation was concerned with comparing the relative properties and characteristics of three specified propellant combinations in regard to propellant acquisition system operation. The purpose of the second evaluation was to determine if the use of a particular propellant combination offered any advantages in regard to propellant acquisition system design. A description of the approaches taken in each evaluation and the results obtained are presented in the following sections.

A. PROPELLANT ACQUISITION SYSTEM EVALUATION

The propellant acquisition system evaluation was based on data and information obtained from the analytical and experimental studies discussed in previous chapters of this report. Six candidate systems, also discussed in previous chapters, were selected for evaluation. These candidates were:

Category A - Compartmented tank;

Category B - Cruciform;

Category C - Standpipe,

Post with fingers,

Vanes,

Vaned standpipe.

The evaluation process consisted of rating the ability of each candidate to satisfy various criteria that included both operational and fabricational considerations. A discussion of the evaluation criteria, the rating system, and the results obtained are presented in this section. 
The candidate system evaluation criteria were generally categorized into two areas: operational, and fabrication and assembly. The operational considerations included those factors associated with the operation and function of the propellant acquisition system. Fabrication and assembly considerations included those factors affecting or influencing manufacture of the system and its installation into the propellant tank. The criteria are discussed in the following paragraphs. In defining criteria, it is desirable that each criterion should be independent of all others. However, it will be apparent that considerable interaction or interdependence will exist between various criteria. This condition results from the fact that the various requirements for the acquisition system are not always independent of one another.

\section{a. Operational Considerations}

Gas-Free Liquid Expulsion - Gas-free liquid expulsion is one of the most important criteria on which the propellant acquisition system is evaluated. This criterion is the primary function or objective of the propellant acquisition system. Influences that were considered in determining the ability of the acquisition system to provide gas-free liquid expulsion were: (1) the manner in which liquid is oriented about the device in low $g$; (2) stability of the liquid orientation; (3) susceptibility to gas ingestion due to dynamic effects such as slosh during settling and suction dip; (4) communication provided between displaced liquid and outlet region; (5) simplicity of outlet design; and (6) relative expulsion efficiency.

Slosh Control - Slosh control is a desirable acquisition system characteristic, not only because of gas-free liquid expulsion considerations, but because of the influence slosh might have on the operation of the vehicle attitude and guidance and control systems. Factors considered in evaluating the acquisition systems slosh control capability were: (1) effectiveness of the device as a baffle; (2) quantity of liquid retained in restart reservoirs; and (3) stability of the interface under to lateral and axial disturbances.

Center of Gravity Control - The ability of the propellant acquisition system to maintain a predictable vehicle $\mathrm{cg}$ during mission coast phases is important in order to minimize attitude control system requirements. It is also important that the system maintain uniform or symmetrical propellant distribution during the settling that occurs when the main engine is started. Unsymmetrical settling 
could cause dynamic loads in excess of guidance and control system capabilities. Factors that affect the cg control provided by the acquisition system invole the quantity of liquid retained in restart reservoirs, the bubble centering and retention capability, the actual ullage volume considered, and communication capability.

Emergency Pressure Relief - It is a system requirement that emergency pressure relief be provided the propellant tank. From the standpoint of both chemical reactivity, propellant conservation, and venting efficiency, it is necessary that only gas be vented. Therefore, the propellant acquisition system must provide sufficient bubble orientation to assure that the ullage is always located at the vent port. This requirement would apply to the entire range of ullage volumes expected during the mission.

Thermal Gradient Sensitivity - Normal system heat leaks through tank supports and propellant feed lines may establish thermal gradients across the propellant tank. The propellant acquisition system should be insensitive to these gradients. Considerations in evaluating the ability of the acquisition system to tolerate thermal gradients were: (1) quantity of propellant trapped forming heat reservoirs or sinks; (2) thermal effect on capillary pumping method employed; (3) thermal effect on liquid interface stability; and (4) effect of propellant vaporization.

Pressurization Sensitivity - The impact of pressurization system requirements on the evaluation of propellant acquisition system operation included the following considerations: (1) ullage breakup due to inlet gas impingement on interface; (2) regulator operation including maximum flow rate and response time; and (3) bubble orientation to prevent liquid from entering the lines and disrupting regulator and vent value operation.

Ground Cooling Integration - For the oxidizer propellant systems, incorporation of ground cooling of the propellant is required. For each acquisition system concept, it is desirable from an installation standpoint that the coolant circuit be incorporated as part of the device. However, this requirement may complicate the design to the point where manufacturing, cleaning, and even operation may be impaired. Factors such as size of the acquisition device would determine whether cooling coils could be integrated. In those devices employing vanes, it may be possible to use the vane surface as a fin to increase cooling efficiency. 
Operating Flexibility - Evaluation of the operating flexibility of each candidate system was based on the system's ability to tolerate normal variations in operating parameters. Consideration must be given to: (1) sensitivity to contact angle and surface tension degradation; (2) sensitivity to ullage volume variations; (3) changes in propellant properties; (4) capability of withstanding acceleration environments exceeding mission criteria; and (5) sensitivity to normal performance tolerances on parameters, such as thrust, flow rate, and impulse bits.

System Flexibility - System flexibility of a propellant acquisition system, as opposed to operating flexibility, is concerned with the effect of major changes in system requirements. Such changes would include modified burn schedules, repackaging of the propulsion system, tank geometry modifications, and complete mission profile changes.

Relative Development Required - This criterion was based on: (1) previous design, development, and manufacturing experience associated with each concept; (2) the complexity of each conceptual design; and (3) additional testing that is required to prove and develop the concept.

Relative Weight - A relative weight assessment was developed for a11 candidate concepts.

Relative Reliability - A comparison of relative reliability was developed for each candidate concept. The following factors were considered: (1) possible loss of liquid from annulus or trap volumes; (2) susceptability of barriers to dry out; (3) possibility of liquid displacement from outlet; (4) material compatibility problems; (5) potential material failure; (6) pore clogging; and (7) thermal stresses created by low operating temperature.

Structural Integrity - Evaluation of each concept in regard to structural integrity considered the following factors: (1) operational load constraints; (2) vibration sensitivity of the concept; (3) thermal shocks associated with propellant loading; (4) loads created by slosh; and (5) potential structural problems associated with system installation in tank.

Loading and Handling - Loading and handling considerations affecting the acquisition system include those ground operations required to load propellants in the spacecraft and then transport and install the spacecraft on the launch vehicle. Factors to be included in the evaluation are: (1) the necessity of vacuum loading the tanks; (2) oxidizer tank passivation and cleanliness requirements; (3) system cooldown prior to loading; and (4) handling limits such as tip angles.

$\mathrm{V}-4$ 
Flight Acceptance Test Constraints - Since any system selected for design and development must ultimately pass flight acceptance testing, a relative evaluation of the difficulty of each candidate system to successfully complete flight acceptance was made. Various test considerations that might be part of a flight acceptance test program were included in the evaluation. The test areas included system leak checks, proof pressure testing, measurement of system weights, system dimensional checks, verification of system flow pressure losses, and environmental considerations such as vibration and thermal.

\section{b. Fabrication and Assembly Considerations}

Material Limitations - Because of the required ten-year mission life and the reactive nature of the propellants, material characteristics present a severe problem for system fabrication. In evaluating the candidate system concepts in regard to material limitations the following factors were considered: (1) propellant compatibility; (2) material strength limitations particularly in bending; (3) required use of dissimilar metals; and (4) quality assurance of postassembly, integrity, and cleanliness.

Joining Processes - Each candidate system was evaluated in regard to joining processes. An assessment was made as to the type of joints required and also if new joining techniques were required. An estimate of the compatibility of the joining methods used in the design with cleanliness requirements was also considered for each concept. Finally, the inspectability of the joints used in the system design was also considered.

Modular Instalzation - One desirable feature in the design of an acquisition system is that it be capable of installation and removal through a 9-in. port on the propellant tank. An evaluation of the capability of each concept to permit the modular installation concept was made. Factors considered in the modular installation evaluation were: (1) constraints on the size of the device; (2) constraints on tank design; (3) ease of installation and removal; (4) special cleaning requirement; and (5) effect on device reliability.

Cleaning Difficulty - The final consideration in regard to system fabrication and assembly evaluation was cleaning difficulty. Factors included were the number and size of cavities and crevices inherent in the concept design. The number and type of connections and joints used in the concept design were also evaluated. 
2. Candidate Concept Rating

The rating system used to evaluate and compare the candidate concepts was based on a quantitative procedure by which a figure of merit (FOM) was calculated for each concept. The candidate with the highest FOM was the system recommended for further study. This procedure, previously used in completing Contract NAS7-754 (Ref $\mathrm{V}-1)$, is described in the following section.

a. Rating Procedure - The first step in the procedure was to define the necessary criteria to support the evaluation (discussed in preceding paragraphs). The next step was to assign a weighting factor to each of the defined criteria. This weighting factor is a measure of the relative importance of each criterion on a scale from 1 to 10 where 1 is least and 10 is most important. Table V-1 presents the weighting factors established for the evaluation criteria.

Table V-1 Evaluation Criteria Weighting Factors

\begin{tabular}{|l|c|}
\hline Criterion & $\begin{array}{l}\text { Weighting } \\
\text { Factor }\end{array}$ \\
\hline Operational Considerations & \\
1. Gas-Free Liquid Expulsion & 10 \\
2. Slosh Control & 7 \\
3. Center of Gravity Control & 8 \\
4. Emergency Pressure Relief & 9 \\
5. Thermal Gradient Sensitivity & 7 \\
6. Pressurization Sensitivity & 8 \\
7. Ground Cooling Integration & 6 \\
8. Operating Flexibility & 3 \\
9. System Flexibility & 1 \\
10. Relative Development Required & 2 \\
11. Relative Weight & 5 \\
12. Relative Reliability & 10 \\
13. Structural Integrity & 6 \\
14. Loading and Handling & 2 \\
15. Flight Acceptance Test Constraints & \\
\hline Fabrication and Assembly Considerations & \\
1. Material Limitations & 3 \\
2. Joining Processes & 5 \\
3. Modular Installation & \\
4. Cleaning Difficulty & \\
\hline
\end{tabular}


The third step in the procedure was to define rating factors for each candidate concept in regard to its ability to satisfy each of the evaluation criteria. The rating factors were based on a scale of 1 to 10 where 1 indicated the poorest and 10 the best compliance with the criterion requirements. Table V-2 presents the rating factors of each candidate concept as established for each evaluation criterion. These rating factors are discussed in paragraph $b$ on the following page.

Table V-2 Condidate Concept Rating Factors

\begin{tabular}{|c|c|c|c|c|c|c|}
\hline \multirow[b]{3}{*}{ Evaluation Criteria } & \multicolumn{6}{|l|}{ Category } \\
\hline & \multirow{2}{*}{$\begin{array}{l}\text { A } \\
\text { Compart- } \\
\text { mented } \\
\text { Tank }\end{array}$} & \multirow{2}{*}{$\begin{array}{l}\text { B } \\
\text { Cruci- } \\
\text { form }\end{array}$} & \multicolumn{4}{|l|}{$\mathrm{C}$} \\
\hline & & & $\begin{array}{l}\text { Stand- } \\
\text { pipe }\end{array}$ & $\begin{array}{l}\text { Post } \\
\text { with } \\
\text { Fingers }\end{array}$ & Vanes & $\begin{array}{l}\text { Vaned } \\
\text { Stand- } \\
\text { pipe }\end{array}$ \\
\hline \multicolumn{7}{|l|}{ Operational Considerations } \\
\hline $\begin{array}{l}\text { 1. Gas-Free Liquid Expulsion } \\
\text { 2. Slosh Control } \\
\text { 3. Center of Gravity Control } \\
\text { 4. Emergency Pressure Relief } \\
\text { 5. Thermal Gradient Sensi- } \\
\text { tivity } \\
\text { 6. Pressurization Sensitiv- } \\
\text { ity } \\
\text { 7. Ground Cooling Integra- } \\
\text { 8. tion } \\
\text { 9. Sperating Flexibility } \\
\text { 10. Relative Development Re- } \\
\text { 11. Ruired } \\
\text { 12. Relative Weight } \\
\text { 13. Structural Integrity } \\
\text { 14. Loading and Handling } \\
\text { 15. Flight Acceptance Test } \\
\text { Constraints }\end{array}$ & $\begin{array}{r}4 \\
8 \\
2 \\
8 \\
1 \\
10 \\
10 \\
3 \\
3 \\
10 \\
3 \\
1 \\
8 \\
1 \\
7\end{array}$ & $\begin{array}{l}3 \\
3 \\
1 \\
1 \\
5 \\
1 \\
1 \\
7 \\
5 \\
5 \\
2 \\
2 \\
5 \\
6 \\
8 \\
9\end{array}$ & $\begin{array}{r}7 \\
3 \\
5 \\
5 \\
7 \\
\\
5 \\
\\
7 \\
6 \\
6 \\
\\
8 \\
9 \\
7 \\
10 \\
10 \\
10\end{array}$ & $\begin{array}{r}1 \\
1 \\
3 \\
3 \\
2 \\
2 \\
3 \\
\\
5 \\
1 \\
1 \\
\\
1 \\
10 \\
3 \\
7 \\
10\end{array}$ & $\begin{array}{r}8 \\
9 \\
10 \\
10 \\
9 \\
9 \\
9 \\
5 \\
8 \\
8 \\
\\
6 \\
7 \\
9 \\
5 \\
5 \\
3\end{array}$ & $\begin{array}{r}10 \\
10 \\
10 \\
10 \\
\\
10 \\
9 \\
\\
8 \\
10 \\
10 \\
\\
5 \\
6 \\
10 \\
5 \\
5 \\
\\
1\end{array}$ \\
\hline \multicolumn{7}{|l|}{$\begin{array}{l}\text { Fabrication and Assembly } \\
\text { Considerations }\end{array}$} \\
\hline $\begin{array}{l}\text { 1. Material Limitations } \\
\text { 2. Joining Processes } \\
\text { 3. Modular Installation } \\
\text { 4. Cleaning Difficulty }\end{array}$ & $\begin{array}{l}6 \\
3 \\
1 \\
1\end{array}$ & $\begin{array}{l}8 \\
8 \\
9 \\
9\end{array}$ & $\begin{array}{l}10 \\
10 \\
10 \\
10\end{array}$ & $\begin{array}{r}9 \\
10 \\
9 \\
10\end{array}$ & $\begin{array}{l}4 \\
6 \\
7 \\
6\end{array}$ & $\begin{array}{l}4 \\
5 \\
6 \\
5\end{array}$ \\
\hline
\end{tabular}


The final step in the rating process was to calculate the figure of merit (FOM) for each concept. This was done by first calculating the product of all concept rating factors and criterion weighting factors. The FOM for each concept was then obtained by taking the sum of the calculated products. The calculated FOM and the evaluation results are discussed in paragraph $c$ following.

b. Concept Rating Factors - In arriving at the rating factors for each concept, a relative comparison of the six candidate concepts was made for each evaluation criterion. In the following paragraphs, a brief discussion of the factors influencing the rating of concept is given.

Operational Considerations - For gas-free liquid expulsion, the vaned standpipe device was judged best because of its ability to provide interface stability, bubble orientation, and symmetrical propellant settling. In addition, the standpipe provides a trap for holding restart propellant near the outlet. The vane concept was considered almost as good as the vaned standpipe except that it lacks the restart trap volume. A standpipe without vanes was downgraded slightly because it does not provide the interface and bubble orientation stability that the vaned systems do. The compartmented tank was downrated primarily because of the lack of communication means to restore displaced propellant to the outlet trap region. The primary objection to the cruciform system was the large quantity of propellant maintained in the device above the outlet which, during settling, moves rapidly down toward the outlet inducing unsymmetrical settling and possible bubble breakup and gas ingestion. Finally, the post with fingers was rated last because it offered the least interface stability of all the candidates.

In regard to slosh control, the systems employing vanes were again rated highest because of the large surface area available for damping. The vaned standpipe, because of the trap volume, was judged better than the vane system. The compartmented tank was rated high because of the separated propellant volumes and the damping characteristics of perforated plates. The standpipe and cruciforms were considered about equal in slosh damping, both having relatively smal1 surface areas. The post with fingers was judged the poorest because of no slosh damping capability at all.

For cg control, both vaned systems were rated a maximum value of 10 because of the control of the ullage bubble provided. The standpipe was rated next because of its ability to retain a quantity of trapped propellant and some control of the ullage bubble. The post with fingers was rated next because of the limited control 
of the ullage bubble. The compartmented tank and the cruciform were rated last in regard to cg control. The compartmented tank was slightly better because of the division of the propellant into smaller volumes. The major disadvantage to the cruciform was its inherent characteristic of providing a displaced or off-centered ullage bubble.

For emergency relief, the vaned systems were again rated highest because of their ability to maintain the ullage bubble in the vicinity of the vent port. The compartmented tank was rated next highest because of the ullage barrier provided by the design in the top of the tank. The remaining concepts were rated in order: standpipe, post with fingers, and cruciform, based on their relative ability to control the ullage.

The primary consideration in evaluating sensitivity to thermal gradients is the quantity of propellant maintained in a reservoir or heat sink. Based on this consideration, the vaned systems were determined to better fulfill the criterion, with the vaned standpipe being the best because of the trapped volume. The standpipe was judged next best, followed by the cruciform which tended to maintain liquid along the center of the tank. The compartmented tank was rated the system most susceptible to thermal effects. Although a significant reservoir is available to absorb heat leaks, the compartmented tank lacks the communication means necessary to pump liquid into the outlet compartment to replace vaporized propellant. All other concepts have a means of pumping propellant to the outlet region to replace any propellant that may have been vaporized.

The sensitivity of the acquisition system concepts to pressurization system operation is based on similar considerations as those for the emergency pressure relief capability. The compartmented tank in this case was rated above the vaned systems because of ullage volume maintenance at the pressurization inlet port. The ullage volume, in addition to protecting the tank pressure regulator lines from.filling with propellant, also tends to act as a diffuser and prevent high velocity gas impingement on the bulk liquid. The vaned systems, while normally maintaining the ullage orientation, do have the potential risk of allowing liquid to" enter the pressurization lines and interfering with regulator operation. The standpipe was down rated further because the same problem could occur, but to a greater degree. The cruciform was rated lowest because of its inability to control ullage adequately. 
The requirement to integrate ground cooling coils in the oxidizer tank appeared to be best accommodated by the compartmented tank system. The required tubing could be incorporated in the cover plate design as structural members without imparing operation of the trap. Therefore, the compartmented tank system was rated highest. The standpipe with vanes in which the standpipe contained the cooling $f$ low channels was rated as the next best concept. In this arrangement the vanes would act as fins to increase heat transfer surface. The cruciform and the standpipe were judged the same with respect to cooling coil integration. Both concepts could accommodate cooling tubes but would not have much additional surface for heat transfer. The post-with-fingers concept was rated next on the assumption that the fingers would, themselves, be made of tubing. The vaned post concept was rated the same as the fingers because of the uncertainty of being able to integrate tubes in the post of a size sufficient to support the cooling needs.

Operating flexibility of the vaned systems was considered best because these systems have greater stability over the normal operation conditions expected. The vaned standpipe was judged better of the two concepts because of the included trap. The standpipe and the cruciform were selected in that order as the next best. These systems were considered to be more sensitive to acceleration environments than the vaned systems and, therefore, were downgraded somewhat. Compartmented tanks were rated lower because the design is not as flexible as far as ullage variation is concerned. Furthermore, the volume of the propellant trap would tend to limit the number of impulse bits before main propellant settling would be required to refill the trap. The post with fingers was rated last because of the lack of stability under lateral or adverse accelerations.

System flexibility of the concepts were given the same relative ratings as those under the previous operating flexibility criterion. The vaned system concepts were found to be less influenced by changes in mission requirements than the other concepts. Sensitivity to tank volume changes or burn schedules did not impact as greatly on the vane systems as on compartmented tank, which must be designed for operation over a limited range of parameters.

Relative development required for the compartmented tank is less than for the other concepts. Therefore, this concept was given the highest rating. Trap systems have the greatest amount of design, drop tower test, and operational experience of all the concepts. A standpipe, because of its simplicity, would require less development than the remaining concepts. Ratings of the remaining concepts are related to their estimated development requirements. 
The estimated relative weights of the concepts are presented in the ratings. The post-with-fingers concept was lightest while the compartmented tank was heaviest.

In regard to relative reliability, the vaned sys tems were rated highest because of their ability to maintain liquid at the out let under both adverse acceleration and thermal conditions. The vaned standpipe was judged somewhat better because of the trapped liquid within the standpipe. The rating of the standpipe alone was reduced because, without vanes, its liquid holding capacity is reduced. However, it still scored higher than the remaining concepts. The compartmented tanks were given the lowest reliability rating because of potential losses of fluid from the trap due to propellant vaporization, barrier dry out, or pore clogging. All the concepts except the compartmented tank have communication capabilities that allow the pumping of displaced liquid to the outlet.

The structural integrity of the standpipe was considered best because of the simplicity of the design together with its ruggedness to withstand vibrational and thermal shocks. The system was also easiest to install in the propellant tank. The compartmented tank was considered second best because of durability and strength. However, the barriers are more difficult to install in the tank and, therefore, the compartmented tank was downrated slightly. The post with fingers was rated as third in regard to structural integrity. Because of small surface areas involved, the effects of slosh is negligible on this concept. Thermal effects should also be insignificant. However, because the fingers are cantilevered rods, some vibrational effects may be significant. The cruciform system was rated about the same as the post with fingers. The cruciform does not have the vibrational problem associated with the post with fingers because it is fastened to the tank at both ends. However, the fact that the device is attached at both ends does introduce problems associated with thermal expansion, particularly in the oxidizer tank where significant temperature differences will occur during cooldown and propellant loading. For this reason, the cruciform was rated slightly lower than the fingers. The vaned systems were rated equal but lower than the other concepts. The low rating was due to slosh loading on vane surfaces, possible vibration sensitivity, and tank installation problems.

In regard to loading and handling, the standpipe and post-withfinger concepts were given high ratings because they are relatively insensitive to the propellant loading requirements, passivation does not appear to be a difficult problem, and they do not require handling limits such as tip angles. The cruciform system was downrated slightly because of possible thermal expansion problems during cooldown and propellant loading. Passivation of the cruciform 
may also be more difficult because of more corners and welds in the design. Vane systems were downrated because of passivation and cleanliness problems associated with the oxidizer requirements. The vane systems have large surfaces and many corners that are difficult to clean and passivate. The compartmented tank was rated last because of cleaning and passivating problems, possible need for vacuum loading of the tank, and susceptibility of partially emptying propellant traps if the system is tipped during installation on the launch vehicle.

In regard to flight acceptance testing, the standpipe, cruciform, post with fingers, and compartmented tank were all rated high and in that order. The standpipe was rated highest because of its simplicity in both design and operation. The cruciform and compartmented tank were downrated because the greater complexity of these systems would result in some difficulty in verifying weight, dimensional, and flow characteristics. Vibrational testing requirements may create problems for flight acceptance of the postwith-finger concept. The vaned systems were rated lowest because they are the most complex in design. Dimensional checks are more difficult because of a large number of tolerances involved in the design.

Fabrication and Assembly Considerations - In regard to material limitations, the standpipe was rated best because of its design simplicity and ease of operation. The post-with-fingers and cruciform concepts were rated next in that order, also because of their simplicity. None of these three systems require the use of dissimilar metals. The compartmented tank was downrated because the perforated plate would complicate postassembly cleaning and structural integrity. The vaned systems were rated lowest because of the bending problems associated with installation and removal. The resulting bending stresses impose severe restrictions on material and material thickness required in the design.

The different joining processes for each concept were evaluated. Both the standpipe and post-with-finger concepts were given the maximum rating because they require the least number of joints. Furthermore, there are no requirements for development of new joining techniques. The cruciform was rated slightly lower because of the necessity to attach both ends of the device to the propellant tank. The vaned systems were rated next with the vanes concept given a little higher rating than the vaned standpipe. The difference in rating is attributed to the more complicated design of the vaned standpipe, which requires more joints. The compartmented tank was rated last because of the large number of welds in the design. The vaned systems and the compartmented tank have joints that might be difficult to inspect.

$\mathrm{V}-12$ 
In regard to modular installation, the standpipe was judged best because it can be installed and removed through the 9-in. hole without any major difficulty. The system is reusable and interchangeable with other propellant tanks. The cruciform and postwith-fingers concepts were next in rating. Both systems require a more complicated installation procedure. The cruciform system must be attached at both ends of the tank while the fingers must be bent or sprung slightly to enter the tank. The vaned systems were rated next in ability to comply with modular installation. The major difficulty with both vanes and vaned standpipes is that special tooling must be developed to roll and unroll the vanes without permanent bending or tearing. The installation procedures required by these systems also has a significant influence on the design of the tank outlet. The vanes were considered easier to install than the vaned standpipe and, therefore, was given a higher rating. The compartmented tank system could not use a modular installation concept and, therefore, was given the lowest rating.

Rating of the systems in regard to cleaning difficulty indicated that the standpipe and post-with-fingers concepts provided the fewest cavities and cracks in which to accumulate contaminants. They also have the fewest number of joints and connections. The cruciform was rated slightly lower because of a mechanical connection at the forward end of the propellant tank. The mechanical connection would present a cleaning problem. Ratings of the vaned systems were significantly lower because of the cavities and cracks inherent in the design. The vanes were given a slightly higher rating than the vaned standpipe because the design is somewhat simpler. The compartmented tank was given the lowest rating because it has the largest number of potential contaminant traps.

c. Rating Results and Concept Recommendations - The figures of merit (FOM) calculated for each concept are presented in Table $\mathrm{V}-3$. Three FOMs were calculated for each concept. The first was an operational FOM based on the operational evaluation criteria. The second was a fabrication FOM based on fabrication and assembly evaluation criteria. The third was an overall concept FOM based on the summation of the first two.

From the operational FOM data given in Table V-3, the vaned standpipe has the highest value while the post-with-fingers concept has the lowest value. Therefore, f rom operational considerations, the vaned standpipe is superior. The vanes concept was second best. 
Table V-3 Calculated Figures of Merit for Candidate Concepts

\begin{tabular}{|c|c|c|c|c|c|c|}
\hline \multirow[b]{3}{*}{ Evaluation Criteria } & \multicolumn{6}{|l|}{ Category } \\
\hline & \multirow{2}{*}{\begin{tabular}{|l} 
A \\
Compart- \\
mented \\
Tank
\end{tabular}} & \multirow{2}{*}{$\begin{array}{l}\text { B } \\
\begin{array}{l}\text { Cruci- } \\
\text { form }\end{array}\end{array}$} & \multicolumn{4}{|l|}{$\mathrm{C}$} \\
\hline & & & $\begin{array}{l}\text { Stand- } \\
\text { pipe }\end{array}$ & $\begin{array}{l}\text { Post } \\
\text { with } \\
\text { Fingers }\end{array}$ & Vanes & $\begin{array}{l}\text { Vaned } \\
\text { Stand- } \\
\text { pipe }\end{array}$ \\
\hline \multicolumn{7}{|l|}{ Operational Considerations } \\
\hline 1. Gas-Free Liquid Expulsion & 40 & 30 & 70 & 10 & 80 & 100 \\
\hline 2. Slosh Control & 56 & 21 & 21 & 7 & 63 & 70 \\
\hline 3. Center of Gravity Control & 16 & 8 & 40 & 24 & 80 & 80 \\
\hline 4. Emergency Pressure Relief & 72 & 9 & 45 & 27 & 90 & 90 \\
\hline $\begin{array}{l}\text { 5. Thermal Gradient Sensi- } \\
\text { tivity }\end{array}$ & 7 & 35 & 49 & 14 & 63 & 70 \\
\hline $\begin{array}{l}\text { 6. Pressurization Sensitiv- } \\
\text { ity }\end{array}$ & 80 & 8 & 40 & 24 & 72 & 72 \\
\hline $\begin{array}{l}\text { 7. Ground Cooling Integra- } \\
\text { tion }\end{array}$ & 60 & 42 & 42 & 30 & 30 & 48 \\
\hline 8. Operating Flexibility & 9 & 15 & 18 & 3 & 24 & 30 \\
\hline 9. System Flexibility & 3 & 5 & 6 & 1 & 8 & 10 \\
\hline $\begin{array}{l}\text { 10. Relative Development } \\
\text { Required }\end{array}$ & 20 & 4 & 16 & 2 & 12 & 10 \\
\hline 11. Relative Weight & 15 & 40 & 45 & 50 & 35 & 30 \\
\hline 12.1 Relative Reliability & 10 & 50 & 70 & 30 & 90 & 100 \\
\hline 13. Structural Integrity & 48 & 36 & 60 & 42 & 30 & 30 \\
\hline 14. Loading and Handling & 4 & 32 & 40 & 40 & 20 & 20 \\
\hline $\begin{array}{l}\text { 15. Flight Acceptance Test } \\
\text { Constraints }\end{array}$ & 14 & 18 & 20 & 16 & 6 & 2 \\
\hline Operational FOM & 454 & 353 & 582 & 320 & 703 & 762 \\
\hline \multicolumn{7}{|l|}{$\begin{array}{l}\text { Fabrication and Assembly } \\
\text { Considerations }\end{array}$} \\
\hline 1. Material Limitations & 18 & 24 & 30 & 27 & 12 & 12 \\
\hline 2. Joining Processes & 9 & 24 & 30 & 30 & 18 & 15 \\
\hline 3. Modular Installation & 7 & 63 & 70 & 63 & 49 & 42 \\
\hline 4. Cleaning Difficulty & 5 & 45 & 50 & 50 & 30 & 25 \\
\hline Fabrication FOM & 39 & 156 & 180 & 170 & 109 & 94 \\
\hline Concept Figure of Merit & 493 & 509 & 762 & 490 & 812 & 856 \\
\hline
\end{tabular}

$\mathrm{V}-14$ 
In regard to fabrication and assembly, the standpipe concept had the highest FOM and was the most desirable. In that respect the vaned standpipe system rated next to last as far as fabrication and assembly considerations were concerned. The low rating was attributed to the concept's design complexity.

The overall concept FOM for the vaned standpipe was the highest. The high operational rating of this concept offset the lower fabrication FOM rating. The lowest overall FOM was given to the postwith-fingers concept. Although fabrication and assembly ratings of the finger concept were high, the overall concept FOM was low because of poor performance.

The overall ranking of the candidate concepts as defined by the concept FOM is:

1) Standpipe with vanes;

2) Vanes;

3) Standpipe;
4) Cruciform;

5) Compartmented tank;

6) Post with fingers.

Based on these evaluation results, the vaned standpipe was recommended to JPL for approval as the preferred concept. Approval of the concept recommendation was received on April 26, 1972.

B. PROPELLANT COMBINATION COMPARISON

The propellant combinations specified for evaluation were Flox/ monomethylhydrazine, fluorine/hydrazine, and oxygen difluoride/ diborane. In these combinations both earth-storable and spacestorable fuels were used. To compare earth- and space-storable oxidizers as a matter of interest, nitrogen tetroxide was also included in the evaluation. The performance factors included in the evaluation were propellant physical property variations, liquid-gas interface stability, capillary pumping capability, ullage variations with temperature, and tank pressure variations from ullage and temperature changes. Each of these considerations are discussed in the following subsections. 
1. Propellant Property Comparison

The first step in the evaluation process was to assemble over the applicable temperature ranges those propellant physical properties pertinent to acquisition device performance. These properties, in addition to providing a means of propellant comparison, were also used in calculations supporting other evaluations. Table V-4 presents the assembled data for each propellant. In general, space storable properties tend to vary more over their applicable temperature ranges than do earth storables. Therefore, it would be expected that performance of a propellant acquisition device would vary more when used with space storables than with earth storables. One of the most important parameters affecting design and operation of a propellant acquisition system is the propellant surface tension. It is desirable that this parameter be as large as possible. Table V-4 shows that space storable fuels and oxidizers have lower surface tension properties and would, therefore, present a more difficult design problem. Other comparisons involving combinations of propellant properties will be discussed in the following subsections dealing with interface stability and capillary pumping characteristics.

2. Gas-Liquid Interface Stability

In any propellant acquisition system using propellant surface tension to position ullage volumes in low-g, the stability of the resulting liquid/gas interface is indicated by the dimensionless Bond number:

Bo $=\frac{\rho a R^{2}}{\sigma}$

where

$\rho=$ propellant density,

$a$ = system acceleration,

$R=$ characteristic system dimension such as tank radius,

$\sigma=$ propellant surface tension, 


\begin{tabular}{|c|c|c|c|c|c|c|c|c|}
\hline 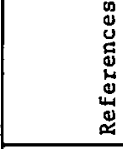 & $*$ & $\mid \begin{array}{l}3 \\
3 \\
i \\
i \\
z\end{array}$ & 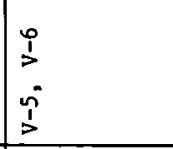 & $\begin{array}{l}0 \\
\vdots \\
\vdots \\
\vdots \\
j\end{array}$ & $\stackrel{o}{i}$ & 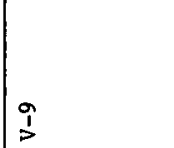 & $\frac{7}{3}$ & 号 \\
\hline 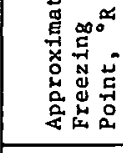 & & : & $\infty$ & $\tilde{\xi}$ & gू & $\stackrel{\sim}{q}$ & $\Xi$ & 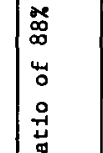 \\
\hline 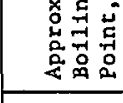 & & $\lg _{g}^{+}$ & $\stackrel{\overbrace{}}{\beth}$ & 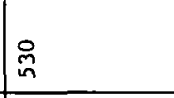 & 高 & $\stackrel{\leftrightarrow}{\Omega}$ & $\stackrel{\sim}{\mu}$ & $\mid$ \\
\hline 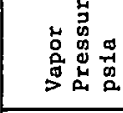 & $\begin{array}{c}0 \\
0 \\
0\end{array}$ & 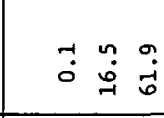 & 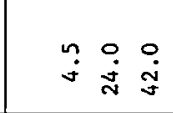 & 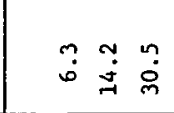 & : & 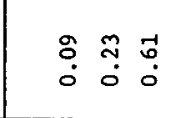 & 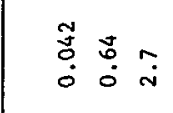 & 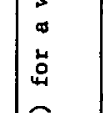 \\
\hline 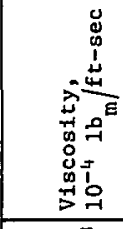 & 象 & 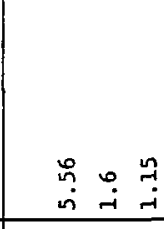 & 乎 & 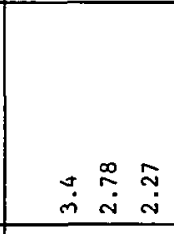 & 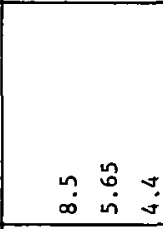 & 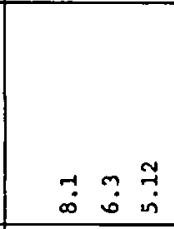 & 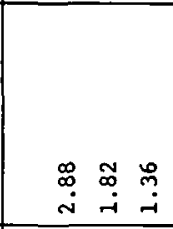 & 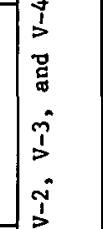 \\
\hline 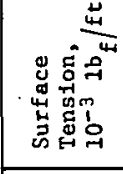 & 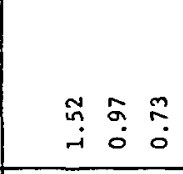 & 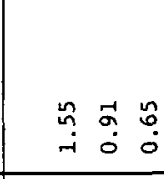 & 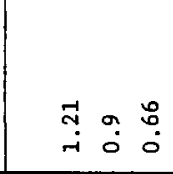 & 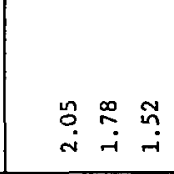 & 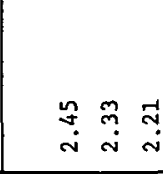 & 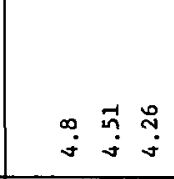 & نج & $\mid$ \\
\hline 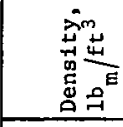 & 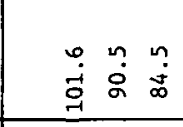 & 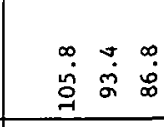 & 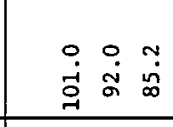 & 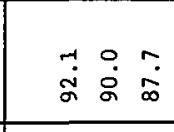 & 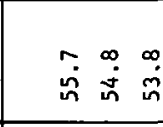 & 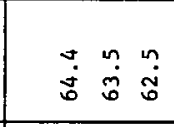 & 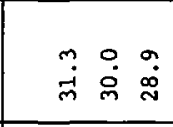 & 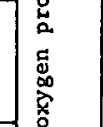 \\
\hline 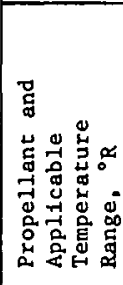 & $g_{\tilde{u}}^{\circ}$ & 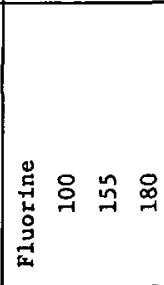 & 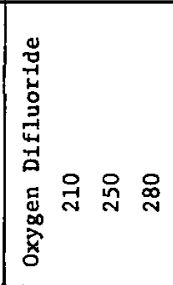 & 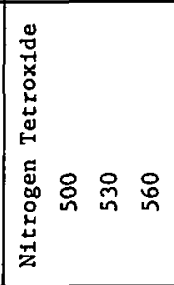 & 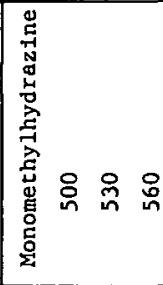 & 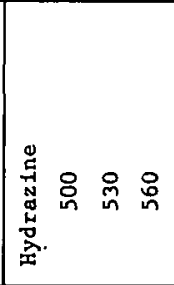 & 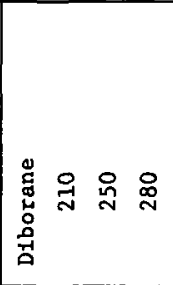 & 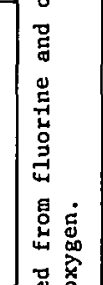 \\
\hline & 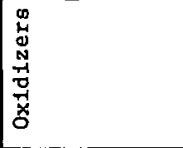 & & & & $\frac{g}{d}$ & & & 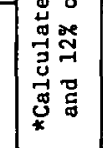 \\
\hline
\end{tabular}


To evaluate and compare the effect of propellant properties on the Bond number and, therefore, the liquid/gas interface stability, all terms not associated with fluid properties were lumped into a constant, $\mathrm{K}$, as follows:

Bo $-K\left(\frac{\rho}{\sigma}\right)$.

The parameter, $\rho / \sigma$, called the interface stability index, is defined by the equation. Evaluation of this parameter provides a way of comparing interface stability for different propellants and propellant combinations on the basis of fluid properties only.

The interface stability index was calculated for each candidate propellant and nitrogen tetroxide at the nominal operating temperature. The results are summarized in $\mathrm{T}$ able $\mathrm{V}-5$.

Table V-5 Interface Stability Index

\begin{tabular}{|c|c|c|c|c|c|c|c|}
\hline & \multicolumn{4}{|c|}{ Oxidizers } & \multicolumn{3}{|l|}{ Fuels } \\
\hline & Flox & Fluorine & $\begin{array}{l}\text { Oxygen } \\
\text { Difluoride }\end{array}$ & $\begin{array}{l}\text { Nitrogen } \\
\text { Tetroxide }\end{array}$ & $\begin{array}{l}\text { Monomethy } 1 \\
\text { Hydrazine }\end{array}$ & Hydrazine & Diborane \\
\hline $\begin{array}{l}\text { Tempera- } \\
\text { ture, }{ }^{\circ} \mathrm{R} \\
\text { Interface } \\
\text { Stability } \\
\text { Index, * } \\
10^{4} 1 \mathrm{~b}_{\mathrm{m}} / \\
\left(1 \mathrm{~b}_{\mathrm{f}}-\mathrm{ft}^{2}\right)\end{array}$ & 155 & 10.4 & 250 & 5.05 & 530 & 530 & $\begin{array}{c}250 \\
2.25\end{array}$ \\
\hline
\end{tabular}

It is desirable that the stability index be as low as possible since a low index value would permit higher accelerations while maintaining a stable interface. The results in Table V-5 indicate that space storable oxidizers have stability indexes approximately twice that of the earth-storable nitrogen tetroxide. No such generalization can be made for fuels because monomethylhydrazine and diborane stability indexes are about the same while hydrazine has a significantly smaller index. The fuel stability indexes are less than those of the oxidizers. 
To compare the interface stability characteristics of the propellant combinations, the sum of oxidizer and fuel indexes for each combination was obtained from the data in Table V-5 and is presented below.

Propellant Combination

Summation of Interface Stability Indexes

F1Ox/MMH

$11.9 \times 10^{4}$

$\mathrm{F}_{2} / \mathrm{N}_{2} \mathrm{H}_{4}$

$11.9 \times 10^{4}$

$\mathrm{OF}_{2} / \mathrm{B}_{2} \mathrm{H}_{6}$

$12.5 \times 10^{4}$

The above data indicate that either Flox/MMH or $\mathrm{F}_{2} / \mathrm{N}_{2} \mathrm{H}_{4}$ propellant combinations would be preferable from interface stability considerations.

Calculations of interface stability indexes were also made for each propellant over the operating temperature ranges presented in $\mathrm{Tab} l \mathrm{e}$ V-4. These calculations were made to assess the effect of temperature on interface stability. The resulting stability indexes are presented in Fig. $\mathrm{V}-1$ and $\mathrm{V}-2$ for oxidizers and fuels, respectively. Figure $V-1$ shows that the interface stability of the earth-storable nitrogen tetroxide is significantly higher (lower index) than that for either of the space storable oxidizers. Furthermore, the sensitivity of the index to propellant temperature changes is much greater for the space storables than it is for $\mathrm{N}_{2} \mathrm{O}_{4}$. Thus, propellant acquisition devices, specifically designed for use with earth-storable $\mathrm{N}_{2} \mathrm{O}_{4}$ may not provide sufficient liquid/gas interface stability if used with space-storable propellants. The large variation of the stability index with temperature for the space-storable propellants also implies that the acquisition device should be designed for the maximum propellant temperature rather than a nominal value. This criterion would prevent the system Bond number from exceeding a maximum allowable value for stable interface conditions if the propellant temperature should rise above the nominal operating temperature. A similar criterion should probably be applied to earth-storable oxidizers although the temperature effect on the stability index is not nearly as great as the space-storable oxidizers. 


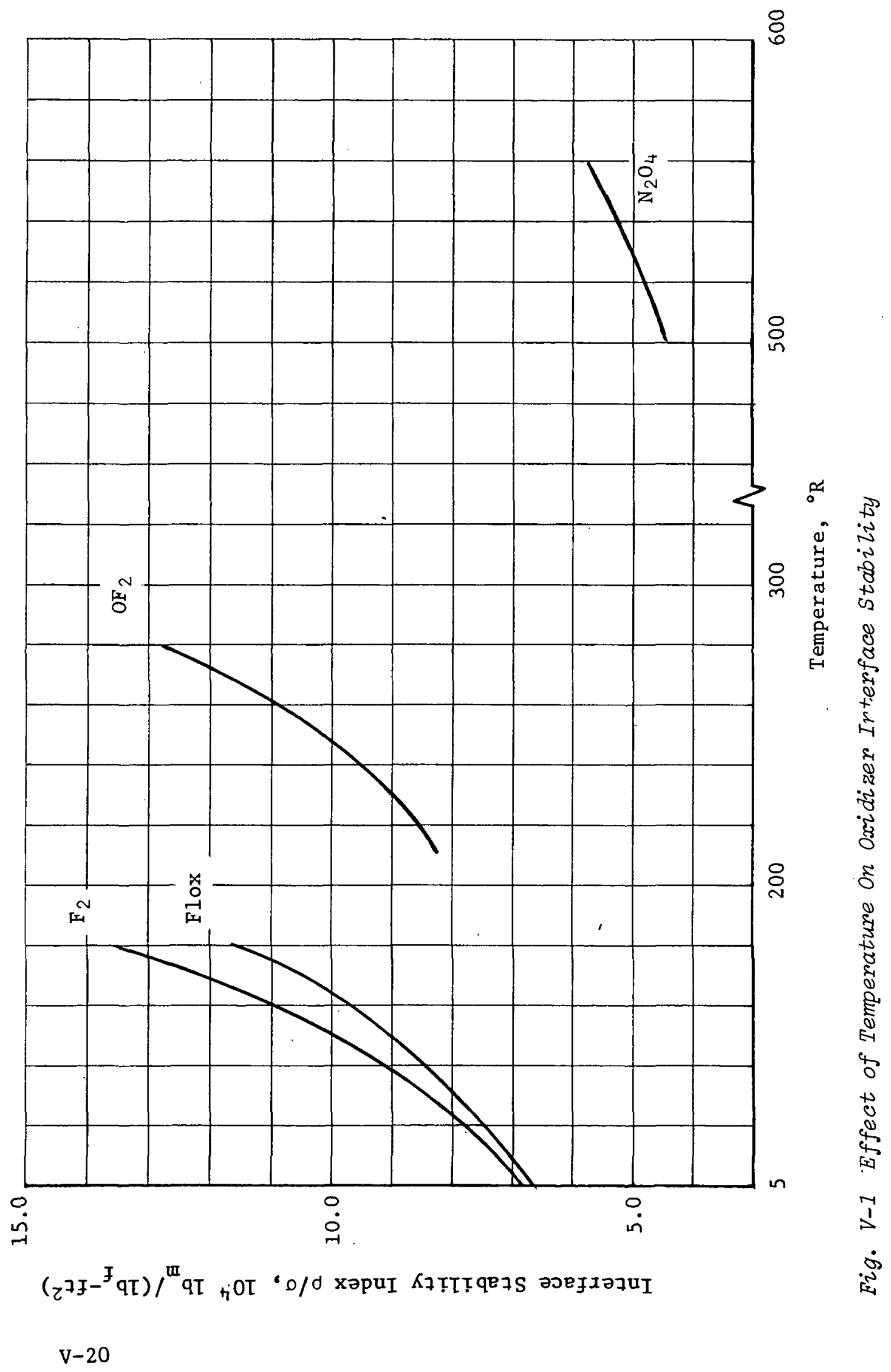




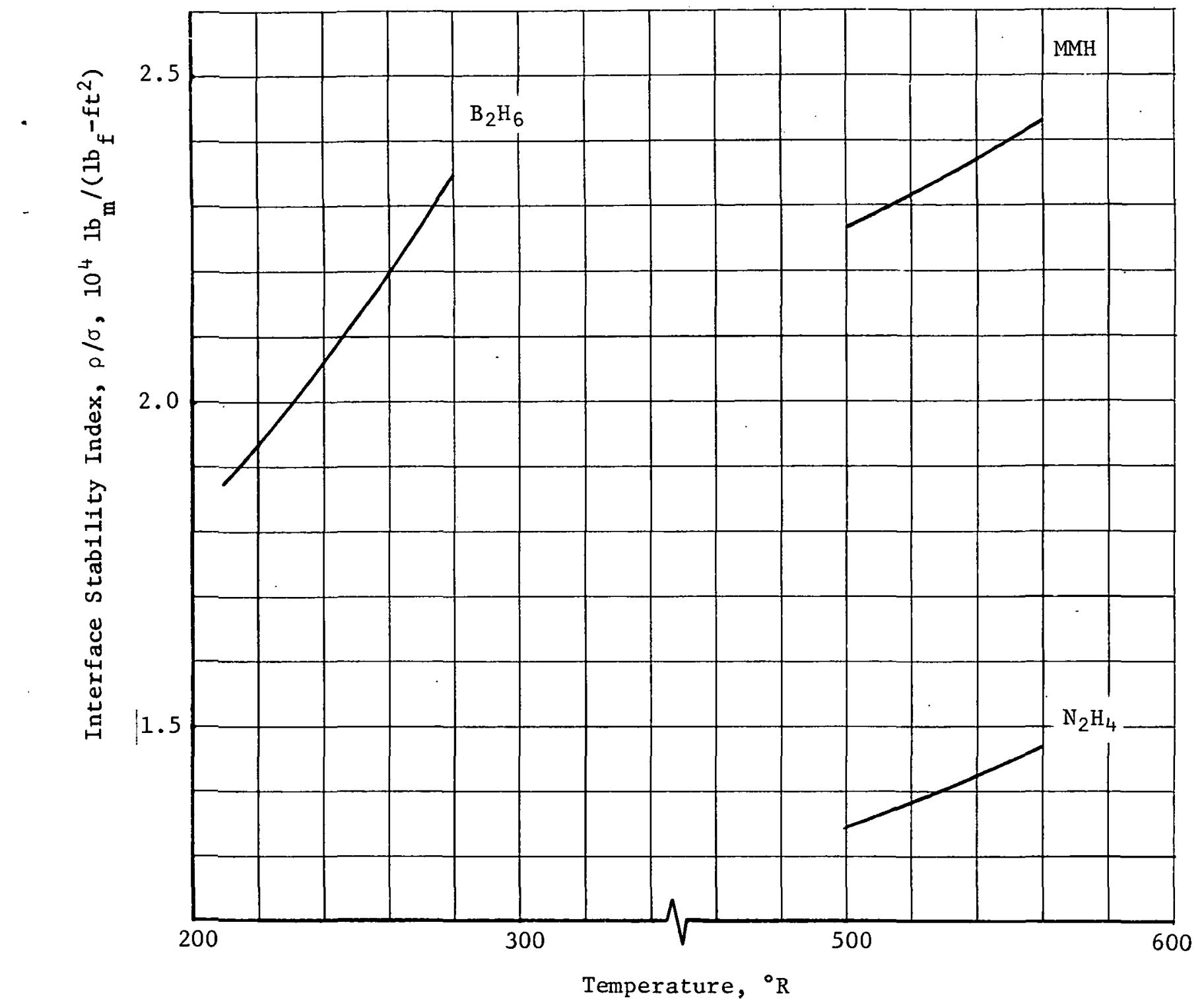

Fig. V-2 Effect of Temperature on Fuel Interface Stability 
The interface stability indexes for the fuels are shown in Fig. $\mathrm{V}-2$. The magnitude of the stability index of space-storable diborane and earth-storable monomethyl hydrazine is about the same while that for earth-storable hydrazine is about $40 \%$ less. Therefore, no generalizations can be made regarding relative levels of earth-storable and space-storable indexes. As far as temperature sensitivity is concerned, the space storables again exhibit a greater variation with temperature than do earth storables. The same temperature design criterion, previously discussed for the oxidizers, would apply to the fuels.

3. Capillary Pumping Index

To determine the effect of propellant properties on capillary pumping capability, the Hagen Poiseuille equation for laminar flow (Ref V-11) was combined with the Young-Laplace equation to produce a characteristic equation containing all pertinent fluid properties controlling capillary flow. The steps and assumptions made in the development of the characteristic capillary pumping equation were as follows. The Hagen-Poiseuille equation is given as:

$\mathrm{Q}=\frac{\pi\left(\mathrm{P}_{0}-\mathrm{P}_{z}\right) \mathrm{R}^{4}}{4 \mu \mathrm{z}}$

where

$$
\begin{aligned}
& Q=\text { volumetric flow rate, } \\
& P_{0}=\text { propellant pressure at channel inlet, } \\
& P_{2}=\text { propellant pressure at channel exit, } \\
& R=\text { channel radius, } \\
& \mu=\text { propellant viscosity, } \\
& z=\text { channel length. }
\end{aligned}
$$


Lumping constants and geometric factors, the equation is simplified to:

$Q=C_{1} \frac{\left(P_{0}-P_{2}\right)}{\mu}$.

A modified Young-Laplace equation for pressure difference across the liquid gas interface can be written as:

$P_{G}-P_{L}=K \sigma \cos \theta$

where

$P_{G}=$ gas pressure,

$P_{L}=1$ iquid pressure,

$\mathrm{K}$ = curvature of liquid gas interface,

$\theta=$ propellant contact angle.

Solving for $\mathrm{P}_{\mathrm{L}}$,

$P_{L}=P_{G}-K \sigma \cos \theta$.

Solving for the liquid pressure at the channel outlet and inlet and taking the difference yields:

$P_{0}-P_{z}=\sigma \cos \theta\left[K_{z}-K_{0}\right]$

where

$\mathrm{K}_{\mathrm{z}}=$ interface curvature at channel exit,

$\mathrm{K}_{\mathrm{O}}=$ interface curvature at channel inlet.

It is assumed that the fluid temperature is constant in the channel so that $\sigma$ and $\theta$ are constant. Substituting the pressure difference expression in the flow equation yields,

$Q=C_{1}\left[K_{2}-K_{0}\right]\left[\frac{\sigma \cos \theta}{\mu}\right]$,

which can be rewritten as:

$Q=C_{2}\left[\frac{\sigma \cos \theta}{\mu}\right]$. 
The parameter, $\sigma \cos \theta / \mu$, called the capillary pumping index, is defined by this equation. Since this parameter is a function only of propellant properties, it provides a means of comparing capillary pumping capabilities of different propellants.

Calculations of the capillary pumping indexes were made for the candidate propellants and nitrogen tetroxide at their nominal operating temperatures. The results are presented in Table V-6. The capillary pumping index should be as large as possible because higher pumping rates reduce the time to reorient propellant should displacement from the tank outlet occur. From the table, it can be seen that the nitrogen tetroxide capillary pumping index is approximately 5 to $18 \%$ greater than that for the space-storable oxidizers. For the fuels, the space-storable diborane is from 8 to $45 \%$ greater respectively than $\mathrm{MMH}$ or hydrazine in capillary pumping capability.

Table V-6 Capizlary Pumping Index, Nominal Temperature

\begin{tabular}{|c|c|c|c|c|c|c|c|}
\hline & \multicolumn{4}{|c|}{ Oxidizers } & \multicolumn{3}{|l|}{ Fuels } \\
\hline & Flox & Fluorine & $\begin{array}{l}\text { Oxygen } \\
\text { Difluoride }\end{array}$ & $\begin{array}{l}\text { Nitrogen } \\
\text { Tetroxide }\end{array}$ & $\begin{array}{l}\text { Monomethyl } \\
\text { Hydrazine }\end{array}$ & Hydrazine & Diborane \\
\hline $\begin{array}{l}\text { Tempera- } \\
\text { ture, }{ }^{\circ} \mathrm{R}\end{array}$ & 155 & 155 & 250 & 530 & 530 & 530 & 250 \\
\hline $\begin{array}{l}\text { Capillary } \\
\text { Pumping } \\
\text { Index } \\
\text { (Volume } \\
\text { Basis)* }\end{array}$ & $\begin{array}{l}1.97 \\
\times 10^{2}\end{array}$ & $\begin{array}{l}1.82 \\
\times 10^{2}\end{array}$ & $\begin{array}{l}1.75 \\
\times 10^{2}\end{array}$ & $\begin{array}{l}2.06 \\
\times 10^{2}\end{array}$ & $\begin{array}{l}1.34 \\
\times 10^{2}\end{array}$ & $\begin{array}{l}2.32 \\
\times 10^{2}\end{array}$ & $\begin{array}{l}2.5 \\
\times 10^{2}\end{array}$ \\
\hline
\end{tabular}

The capillary pumping characteristics of each propellant combination were analyzed in a manner similar to that for interface stability. Summation of the capillary pumping indexes for each propellant combination is tabulated below.

Propellant Combination Summation of Capillary Pumping Indexes

Flox/MMH $3.31 \times 10^{4}$

$\mathrm{F}_{2} / \mathrm{N}_{2} \mathrm{H}_{4} \quad 4.14 \times 10^{4}$

$\mathrm{OF}_{2} / \mathrm{B}_{2} \mathrm{H}_{6} \quad 4.25 \times 10^{4}$

The $\mathrm{OF}_{2} / \mathrm{B}_{2} \mathrm{H}_{6}$ propellant combination has a slightly higher pumping index than the $\mathrm{F}_{2} / \mathrm{N}_{2} \mathrm{H}_{4}$ system. 
Capillary pumping indexes were also calculated for each propellant over the operating temperature ranges specified in Table V-4 for contact angles of 0 and $20^{\circ}$. The capillary pumping indexes are plotted in Fig. V-3 and V-4 for the oxidizers and fuels, respectively. Figure $V-3$ shows that the maximum values of the pumping indexes for Flox and fluorine are about the same as that for nitrogen tetroxide while the oxygen difluoride value is somewhat less. However, the variation of the index for fluorine or Flox is much more sensitive to temperature changes than for either oxygen difluoride or nitrogen tetroxide. The magnitude of this variation suggests that propellant communication channels used in Flox or fluorine systems should be designed for the low temperature limit. This criterion is necessary to assure adequate liquid pumping over the entire allowable temperature range. For a given communication channel design, pumping would tend to increase as the propellant temperature increased.

In regard to oxidizer sensitivity to contact angle variations, the nitrogen tetroxide pumping index appears to vary the most for a $20^{\circ}$ contact angle change. This 6 to $7 \%$ variation in pumping index is only slightly larger than the variation due to temperature change.

Analysis of the capillary pumping indexes for the fuels in Fig. V-4 does not indicate any major or significant differences in regard to either temperature or contact angle effects for either space- or earth-storable fuels. Design of communication channels for both space- and earth-storable fuels should be based on minimum operating temperature as discussed above for the oxidizers. The magnitude of the pumping indexes for diborane and hydrazine are the same while monomethyl hydrazine is about half as much as the hydrazine. The difference between the hydrazine and monomethyl hydrazine is primarily attributed to the difference in surface tension of the two fuels.

\section{Ullage Variation with Temperature}

The volume of the ullage bubble is a significant parameter in the design and operation of a propellant acquisition device. The Jupiter mission used in this program as a study guideline employs two short burns during transfer phase from Earth to Jupiter orbit. The transfer phase is approximately two-years long. During this period, heat additions or losses would tend to reduce or increase the ullage because of expansion or contraction of the propellant. 


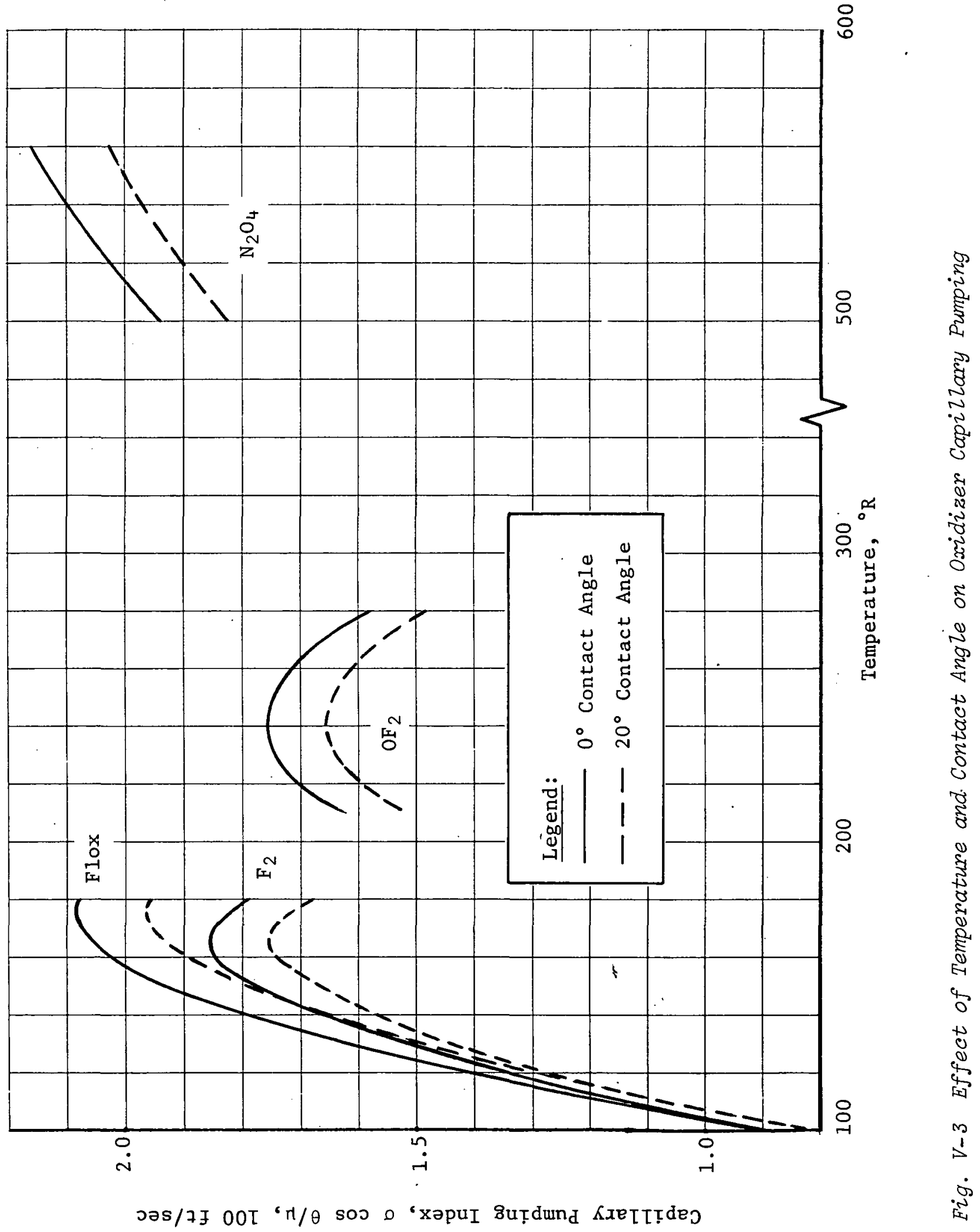




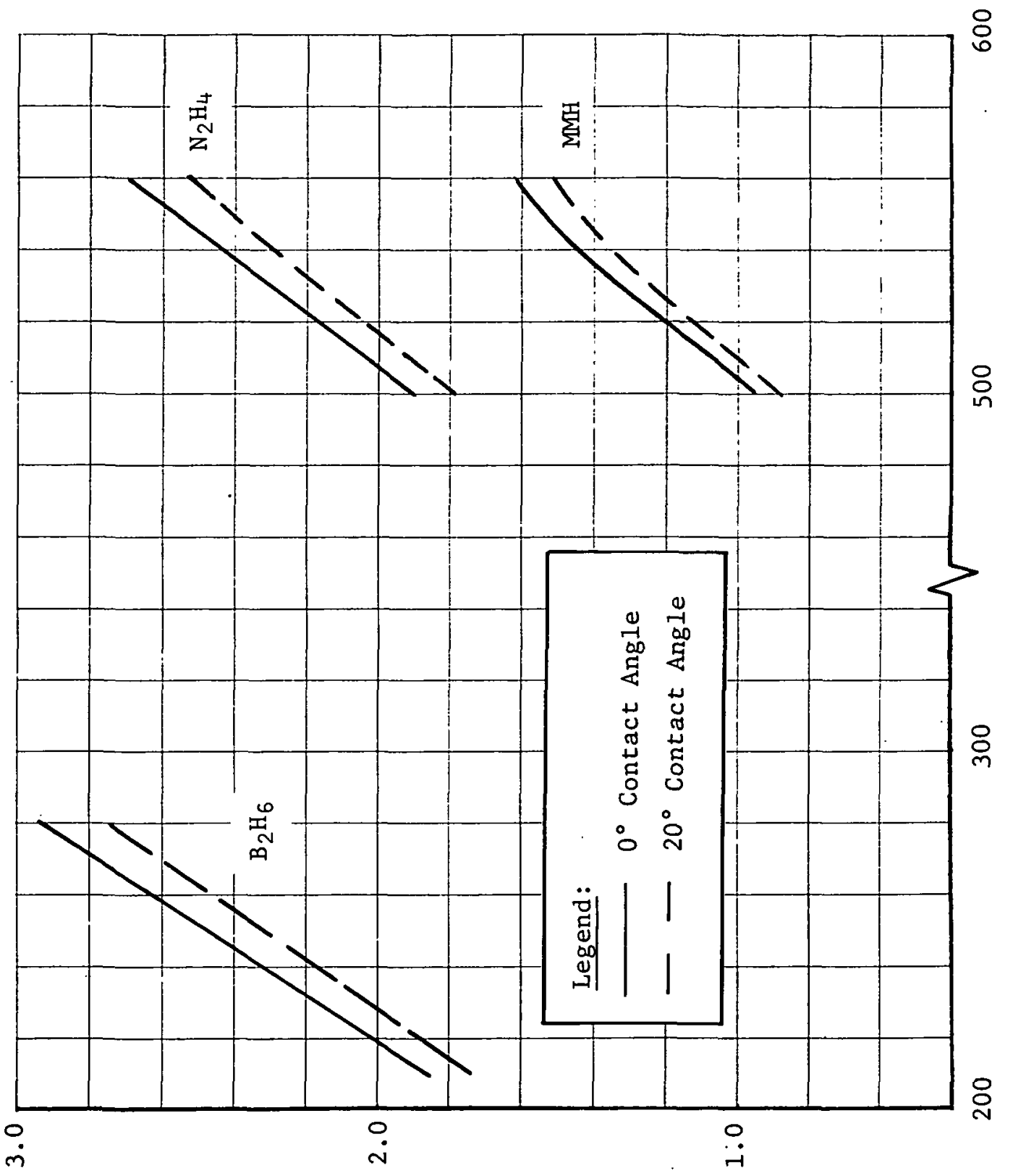

8

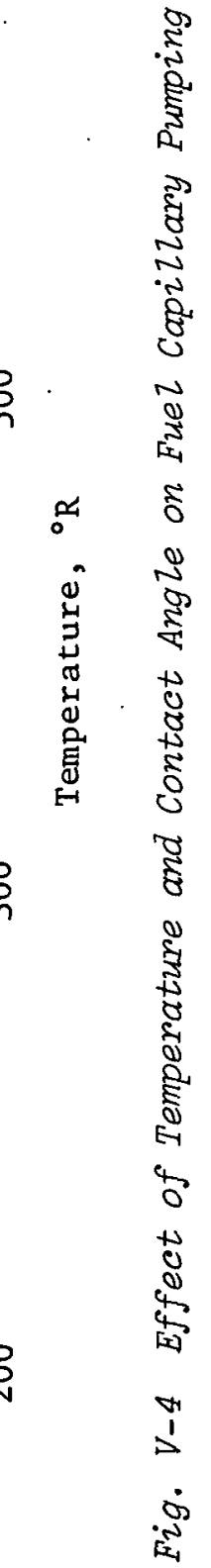


To evaluate the sensitivity of the tank ullage to propellant temperature changes, calculation of ullage fraction changes from an assumed initial ullage fxaction value at the nominal propellant temperature were made over the specified temperature range. The development of the equation used to calculate the ullage fraction as a function of temperature is presented briefly as follows. It is assumed that the mass of the propellant is constant (i.e., vaporization or condensation of propellant during temperature change is neglected). The propellant tank volume is also assumed constant. Equilibrium temperature conditions are also assumed between gas, 1iquid, and tank. Based on these assumptions,

$\mathrm{V}_{\mathrm{T}}=\mathrm{v}_{\mathrm{u}}+\mathrm{v}_{\mathrm{p}}$

where

$\mathrm{V}_{\mathrm{T}}=$ tank volume,

$v_{u}=u 1 l$ age volume,

$\mathrm{V}_{\mathrm{p}}=$ propellant volume.

The propellant volume is:

$\mathrm{V}_{\mathrm{p}}=\mathrm{M}_{\mathrm{p}} / \rho_{\mathrm{p}}$

where

$M_{p}=$ propellant mass,

$\rho_{p}=$ propellant density.

Then,

$v_{T}=v_{u}+\frac{M_{p}}{\rho_{p}}$

Solving for $M_{p}$,

$\mathrm{M}_{\mathrm{p}}=\rho_{\mathrm{p}}\left(\mathrm{V}_{\mathrm{T}}-\mathrm{V}_{\mathrm{u}}\right)$. 
Since $M_{p}$ is assumed constant,

$\rho_{\text {po }}\left(\mathrm{v}_{\mathrm{T}}-\mathrm{v}_{\mathrm{uo}}\right)=\rho_{\mathrm{p}}\left(\mathrm{v}_{\mathrm{T}}-\mathrm{v}_{\mathrm{u}}\right)$

where subscript o refers to nominal conditions. Solving for $V_{u}$, the ullage volume at any temperature,

$v_{u}=v_{T}-\left(\frac{\rho_{p o}}{\rho_{p}}\right)\left(v_{T}-v_{u o}\right)$.

Dividing by the tank volume which is assumed constant,

$\frac{\mathrm{v}_{\mathrm{u}}}{\mathrm{v}_{\mathrm{T}}}=1-\left(\frac{\rho_{\mathrm{po}}}{\rho_{\mathrm{p}}}\right)\left(1-\frac{\mathrm{v}_{\mathrm{uo}}}{\mathrm{V}_{\mathrm{T}}}\right)$.

The ratio of $\mathrm{V}_{\mathrm{u}} / \mathrm{V}_{\mathrm{T}}$ is the ullage fraction at temperature, $\mathrm{T}$, while $\mathrm{V}_{\mathrm{uo}} / \mathrm{V}_{\mathrm{T}}$ is the initial ullage fraction at nominal temperature $\mathrm{T}_{0}$. Values of $\mathrm{V}_{\mathrm{uo}} / \mathrm{V}_{\mathrm{T}}$ of $0.05,0.10,0.15,0.20$, and 0.50 were assumed for each propellant at its nominal temperature, $\mathrm{T}_{0} \cdot$ Various values of $\mathrm{T}$ were assumed over the propellant operating temperature range and the corresponding value of $V_{u} / V_{T}$ computed from the above equation. Figures $\mathrm{V}-5$ and $\mathrm{V}-6$ are plotted values of $\mathrm{V}_{\mathrm{u}} / \mathrm{V}_{\mathrm{T}}$ as a function of temperature for oxidizers and fuels, respectively. Since the Flox and fluorine densities are very nearly equal, the calculated ullage fractions are nearly equal so that only a single set of curves are shown. It can be seen from the Fig. V-5 that the slope of the ullage fraction curves for the space storable are steeper than those for nitrogen tetroxide. Therefore, ullages for the space-storable propellants are more sensitive to temperature variations. It is concluded that space-storable propellants present a more critical design problem for propellant acquisition devices because of the greater potential ullage variations.

In Fig. V-6, the effect of temperature on ullage for the fuels is shown. Only one set of curves is shown for monomethylhydrazine and hydrazine since the density and density variations for the two propellants are about the same. The ullage variation with temperature for both space-storable and earth-storable fuels is about the same so that no distinction can be made regarding propellant acquisition design differences. 


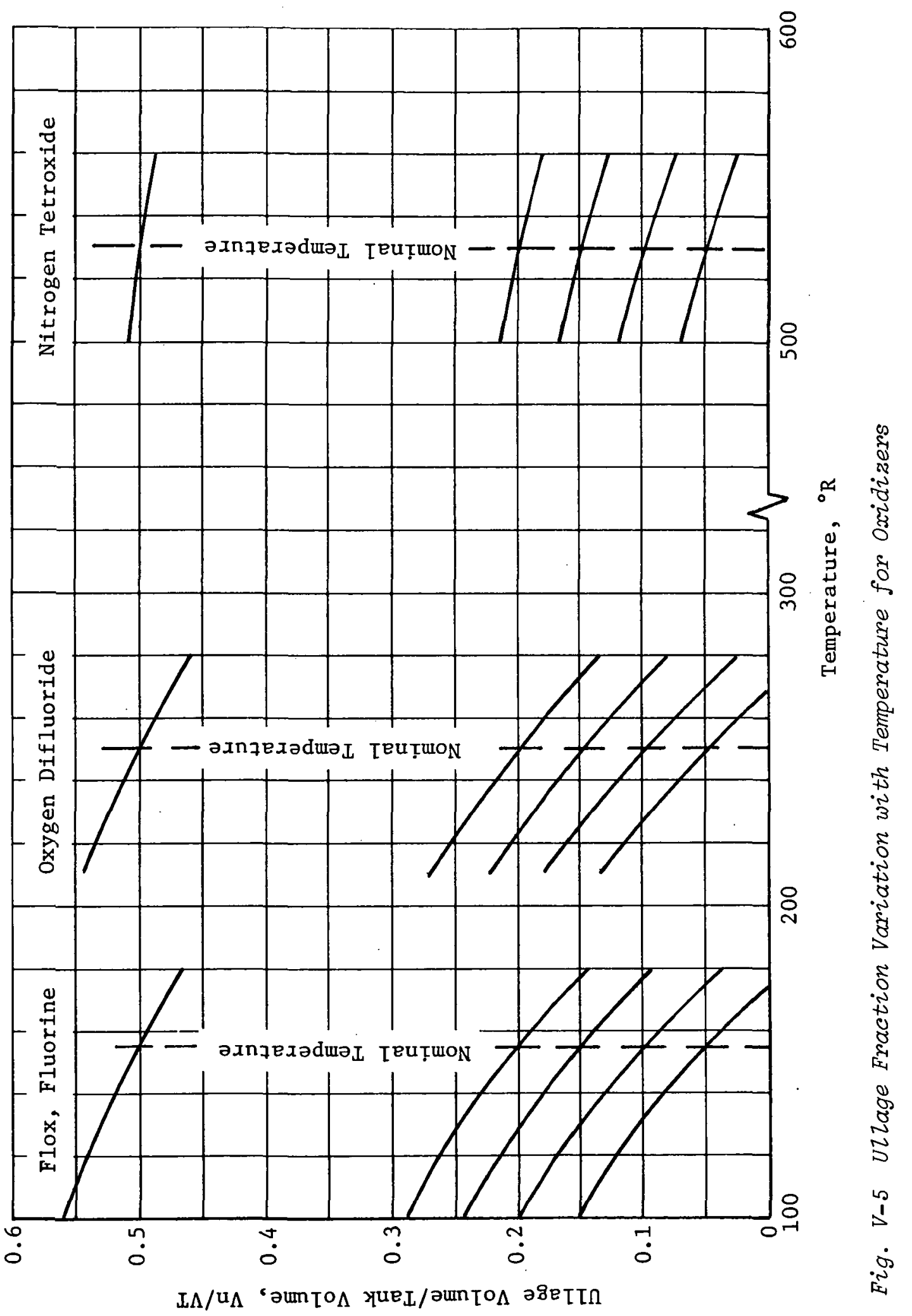




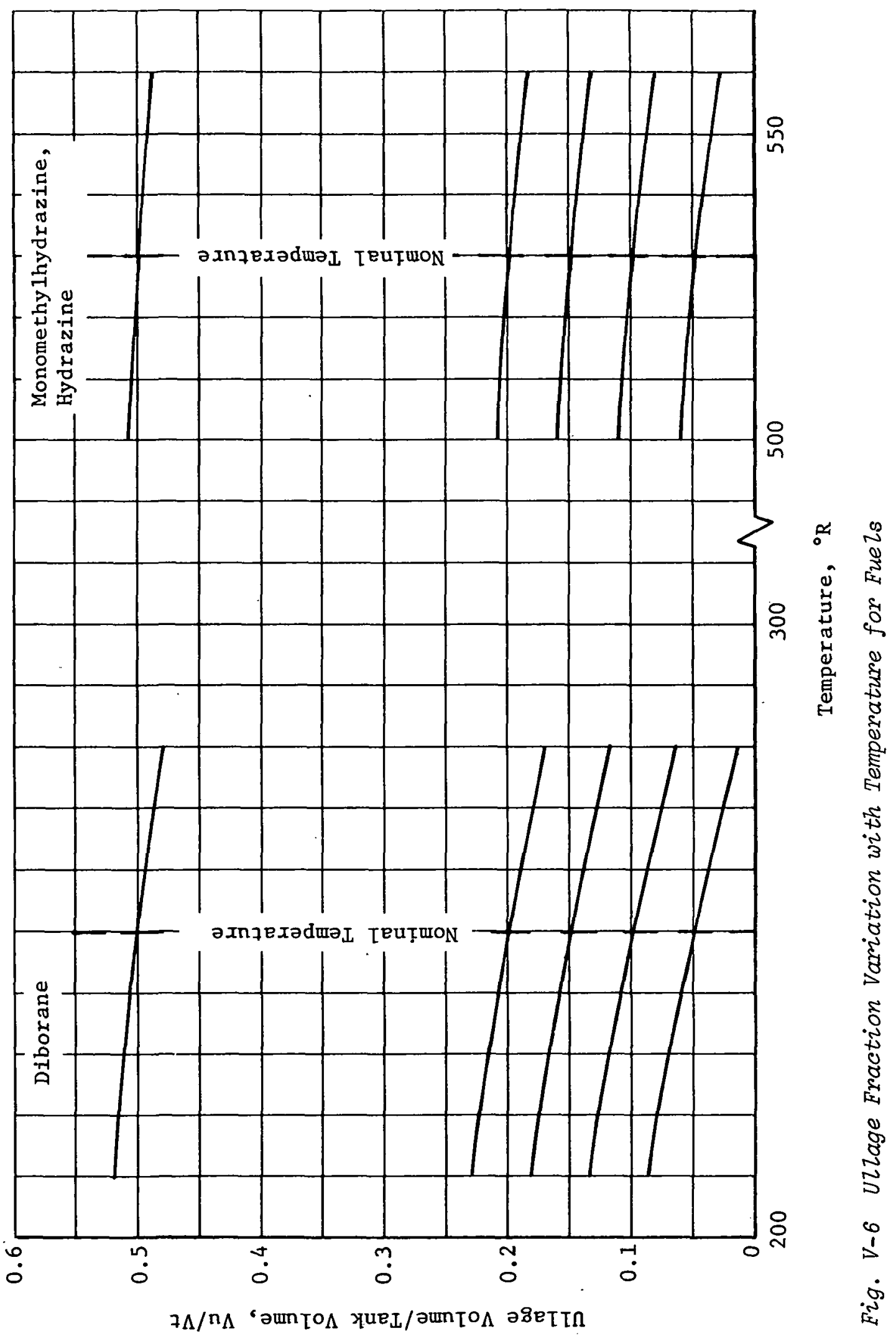


In the previous section, the effect of temperature on ullage volume was discussed. Associated with the ullage and temperature change is a tank pressure change. Tank pressure variations can impact on the design of propellant acquisition systems in several ways. First, if large overpressure conditions develop with temperature changes so that venting is required, the propellant acquisition device must orient the ullage so that gas only is released through the vent port. A second effect on propellant acquisition system performance that results from overpressure conditions is the excessive startup flow rate conditions with associated off-nominal thrust and mixture ratio. These conditions were previously discussed in the section on pressurization system interactions.

The purpose of this study is to evaluate the tank pressure rises that occur when propellant temperature and, therefore, tank ullages vary during the long orbit transfer phase of the mission. The assumptions made in the previous section are also applicable in this study. An additional assumption is that ideal gas behavior is followed. The tank pressure is given by Dalton's Law as:

$P_{T}=P_{G}+P_{V}$

where

$\mathrm{P}_{\mathrm{T}}=$ tank pressure,

$P_{G}=$ noncondensible gas (helium) pressure,

$\mathrm{P}_{\mathrm{V}}=$ propellant vapor pressure.

Assuming no change in noncondensible gas mass and applying the perfect gas equation,

$\frac{\mathrm{P}_{\mathrm{GO}} \mathrm{V}_{\mathrm{uo}}}{\mathrm{T}_{\mathrm{O}}}=\frac{\mathrm{P}_{G} \mathrm{~V}_{\mathrm{u}}}{\mathrm{T}}$

where

$\mathrm{v}_{\mathrm{u}}=$ tank ullage,

$\mathrm{T}=$ system temperature. 
and subscript o refers to nominal condition. Solving for $P_{G}$,

$P_{G}=P_{G O}\left(\frac{T}{T_{0}}\right)\left(\frac{V_{u o}}{V_{u}}\right)$

Dividing $\mathrm{v}_{\mathrm{uo}}$ and $\mathrm{v}_{\mathrm{u}}$ by the tank volume, $\mathrm{V}_{\mathrm{T}}$,

$P_{G}=P_{G O}\left(\frac{T}{T_{0}}\right) \frac{\left(\mathrm{V}_{\mathrm{uo}} / \mathrm{V}_{\mathrm{T}}\right)}{\left(\mathrm{V}_{\mathrm{u}} / \mathrm{V}_{\mathrm{T}}\right)}$

The terms $\mathrm{V}_{\mathrm{uo}} / \mathrm{V}_{\mathrm{T}}$ and $\mathrm{V}_{\mathrm{u}} / \mathrm{V}_{\mathrm{T}}$ are the ullage fractions at $\mathrm{T}_{\mathrm{o}}$ and $\mathrm{T}$, respectively. These values were obtained from Fig. V-5 and V-6.

The propellant vapor pressure is a function of the temperature only. That is,

$P_{V}=P_{V}(T)$

Combining $\mathrm{P}_{G}$ and $\mathrm{P}_{V}$ in the total tank pressure equation,

$P_{T}=P_{G O}\left(\frac{T}{T_{0}}\right) \frac{\left(V_{u_{0}} / V_{T}\right)}{\left(V_{u} / V_{T}\right)}+P_{V}(T)$.

This equation was used to calculate the tank pressures resulting from temperature and ullage changes during orbital transfer. To use this equation, a value for $\mathrm{P}_{G O}$, the partial pressure of the noncondensible gas at nominal temperature, had to be defined. This value was obtained from Dalton's Law assuming a nominal tank pressure of 260 psia and a propellant vapor pressure corresponding to the nominal temperature. The calculated tank pressure versus temperature curves are plotted in Fig. $\mathrm{V}-7$ and $\mathrm{V}-8$ for oxidizers and fuels, respectively. The curves were based on a nominal initial ullage fraction of $0.05,0.10,0.15,0.20$, and 0.50 . Only one set of curves is shown for Flox and fluorine and for monomethylhydrazine and hydrazine. Because of the similarity in density and vapor pressure of the two oxidizers and the two fuels, the calculated pressures were within a few percent of one another. Plotting the results on the scales used in the graph made it difficult to distinguish the curves for each propellant. However, the data are to be used as a relative comparison only so a single curve for the two propellants does indicate trends and characteristics for both. 


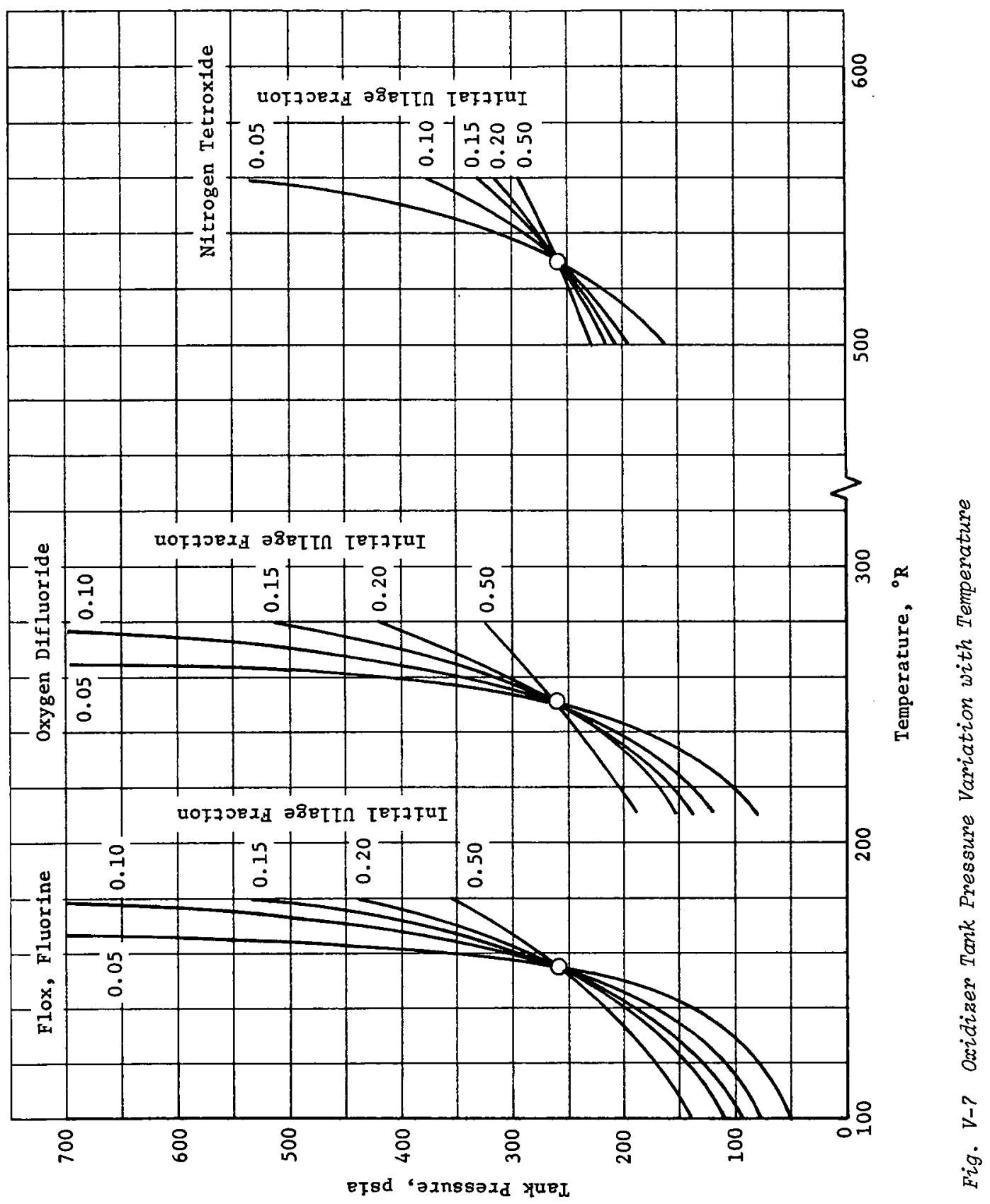




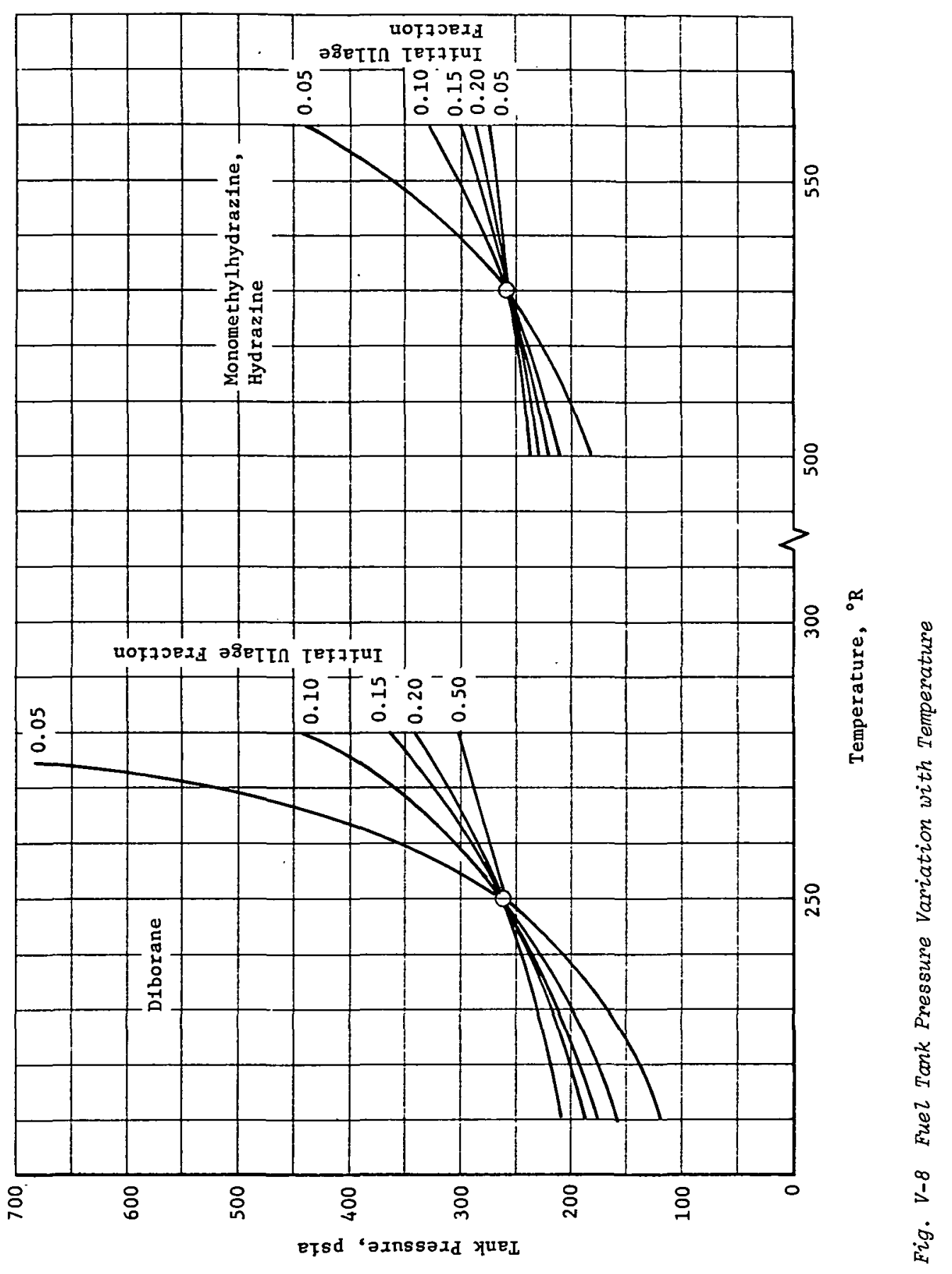


Figure V-7 indicates that the space-storable oxidizers exhibit a greater sensitivity to pressure variations than the earth-storable class. Two factors contribute to this condition: (1) greater expansion of space-storable liquid volumes with temperature, and (2) higher space storable vapor pressures. From the figure, it can be seen that initial ullage fractions less than $20 \%$ in space storable oxidizer tanks create extremely high pressure conditions. To accommodate these pressures, prohibitively heavy tanks would be required. For earth-storable oxidizers, ullage Eractions between 0.05 and 0.10 could be used without the potentiality of extremely high pressures developing.

Comparison of the data for fuels in Fig. V-8 shows that an initial ullage fraction of 0.10 produces maximum tank pressure conditions in the space-storable tanks equivalent to those in earth-storable tanks employing an initial ullage fraction of 0.05 . In general, tank pressures occurring in space-storable fuel tanks are higher than those in earth-storable fuels. However, the differences are not as great as in the oxidizer tanks.

6. Propellant Comparison Conclusions

The evaluations and comparisons showed that no major differences existed between the three specified propellant combinations with respect to propellant acquisition. For propellant nominal operating temperature conditions, the Flox/MMH and $\mathrm{F}_{2} / \mathrm{N}_{2} \mathrm{H}_{4}$ combinations exhibited slightly better interface stability characteristics than the $\mathrm{OF}_{2} / \mathrm{B}_{2} \mathrm{H}_{6}$ combination. However, the $\mathrm{OF}_{2} / \mathrm{B}_{2} \mathrm{H}_{6}$ combination possessed the highest capillary pumping capability. The differences between these characteristics were small so that no distinct recommendations of the best propellant combination for propellant acquisition could be made.

A comparison of individual propellants on the basis of their storability and nominal temperature ranges was also made. This comparison indicated that space-storable propellants would present more difficult design problems in developing propellant acquisition systems. In general, spacerstorable propellants possess lower surface tension properties, possess lower interface stability characteristics, and provide lower capillary pumping capability than do earth storables. Furthermore, the interface stability and capillary pumping ability of space-storable propellants are more sensitive to temperature variations than those for earth storables. 
Ullage volume is a critical parameter in the design of propellant acquisition systems. Because the liquid thermal expansion of space-storable propellants over their normal temperature range is greater than the corresponding expansions of earth-storable propellants, greater variations in ullage volumes with temperature will occur with space storables. Therefore, if the acquisition system is to be designed for a specific range of ullage volume, space-storable propellants present a more difficult design problem because of ullage sensitivity to temperature. 
VI. DETAIL DESIGN OF SELECTED SYSTEM

The various candidate surface tension propellant acquisition devices were analyzed and evaluated to arrive at the one best concept. A standpipe with vanes was the concept most capable of meeting the acquisition system requirements for the baseline spacecraft and mission. In this portion of the program the analysis and design of the standpipe with vanes was continued to further establish the capabilities and performance of the system. Evaluation of the interaction of the propellant acquisition system with the other spacecraft subsystems was also continued. Details concerning the materials of construction, fabrication and installation of the device, testing, cleaning, loading and handling, and mission operation were considered. The result is a preliminary design of the surface tension propellant acquisition system for the baseline Space Storable Propulsion Module and the Jupiter mission. The basic design of this Fruhof-type system, however, is applicable to most envisioned interplanetary spacecraft. The design of the system and its operation are discussed in this chapter.

A. SYSTEM DESIGN

As a result of the effort in this phase of the program, engineering drawings of the Fruhof standpipe with vanes propellant acquisition concept were produced. Figures VI-1 and VI-2 show the concept as it would be applied to a typical oxidizer and fuel tank, respectively. The specific dimensions on the drawings are for the propulsion system using the Flox/MMH propellant combination, but the same devices could be scaled to apply to the system with the $\mathrm{F}_{2} / \mathrm{N}_{2} \mathrm{H}_{4}$ combination.

The drawings represent only a preliminary evaluation of the design concept. The design and analysis were based on typical mission requirements and criteria. Further design, analysis, and definition of requirements would be necessary before the drawings could be upgraded, leading to the actual fabrication of the device. Within the limits of the program, the illustrated devices are believed to best meet the acquisition system operation, integration, and fabrication requirements. The devices will provide gasfree propellant to the engine on demand and will not hinder the 
operation of any spacecraft subsystems, i.e., vent, pressurization, thermal control, ACS. An attempt was made to provide a design that is compatible with a 10-year life requirement and is capable of modular installation or removal through a 9-in.-diameter tank access port. A ground hold cooling system was integrated into the design for the oxidizer tank propellant acquisition system.

Two alternative acquisition system designs are also presented for the oxidizer tank. Rather than integrating the ground hold cooling system into the device, the coolant coils could be mounted on the internal tank wall, as shown in Fig. VI-3. The cooling coils would be welded as part of the tank structure and would also serve as communication channels. The device, however, could still be modularly installed.

If the requirements for modular installation and internal cooling coils were not essential, the same device could be fabricated as an integral part of the propellant tanks, as shown in Fig. VI-4. The device and the communication channel would both be welded to the tank wall during the tank fabrication operation.

Details of the devices are discussed in the following paragraphs. 


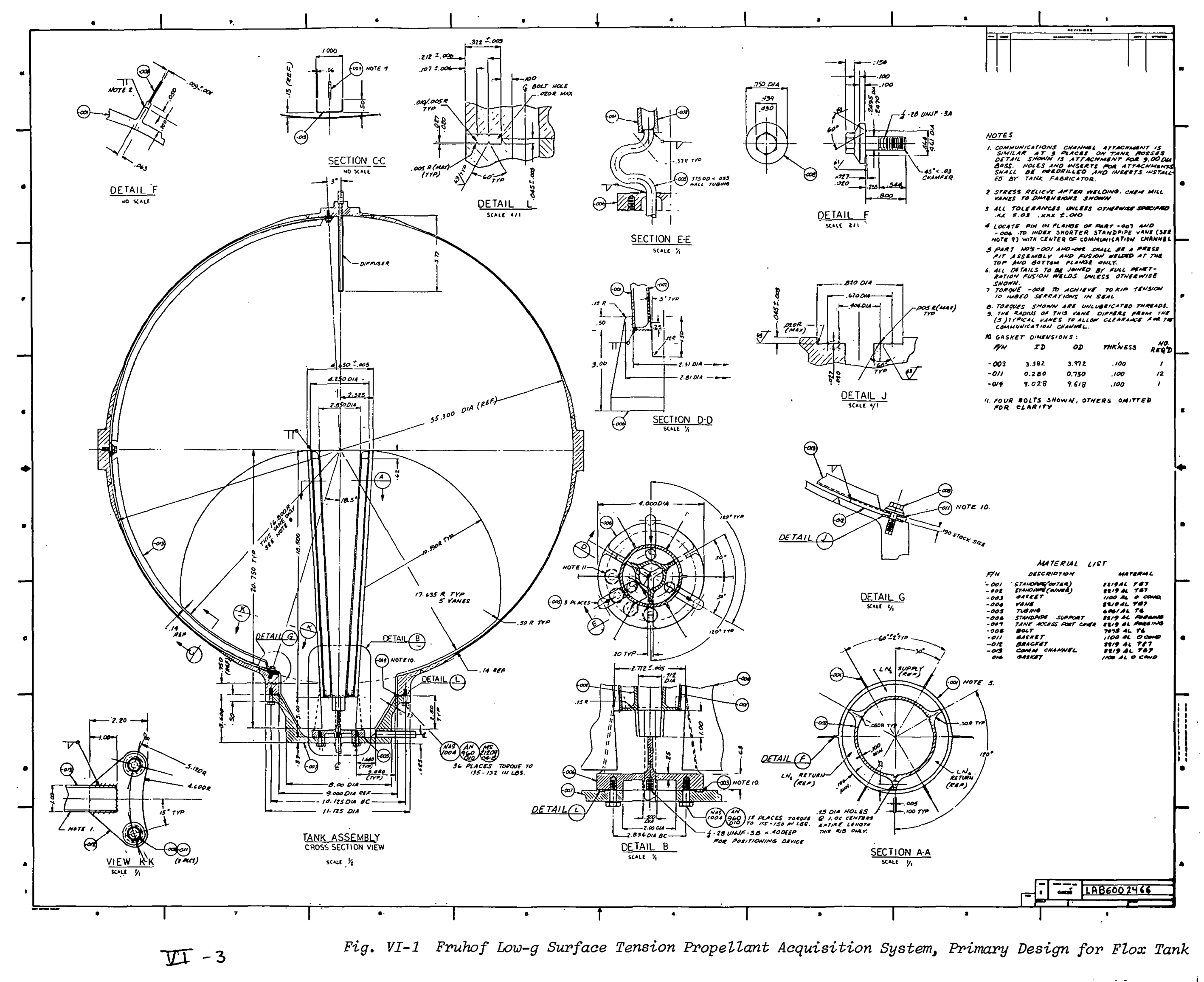

FOLDOUT ERAME | 


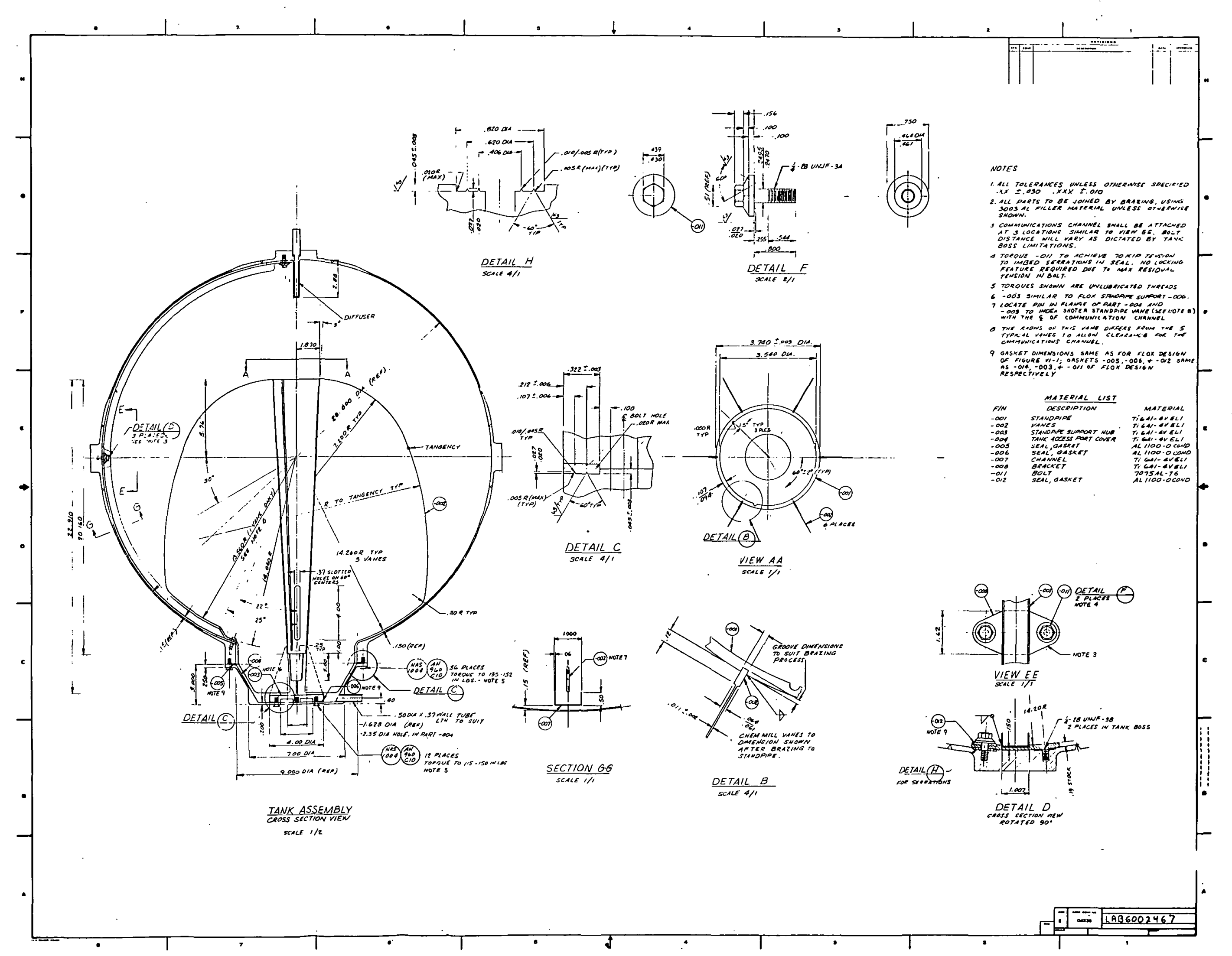

IVE5 Preceding page blank

EOLDOUT FRAME |

FOLDOUT FRAME 2 


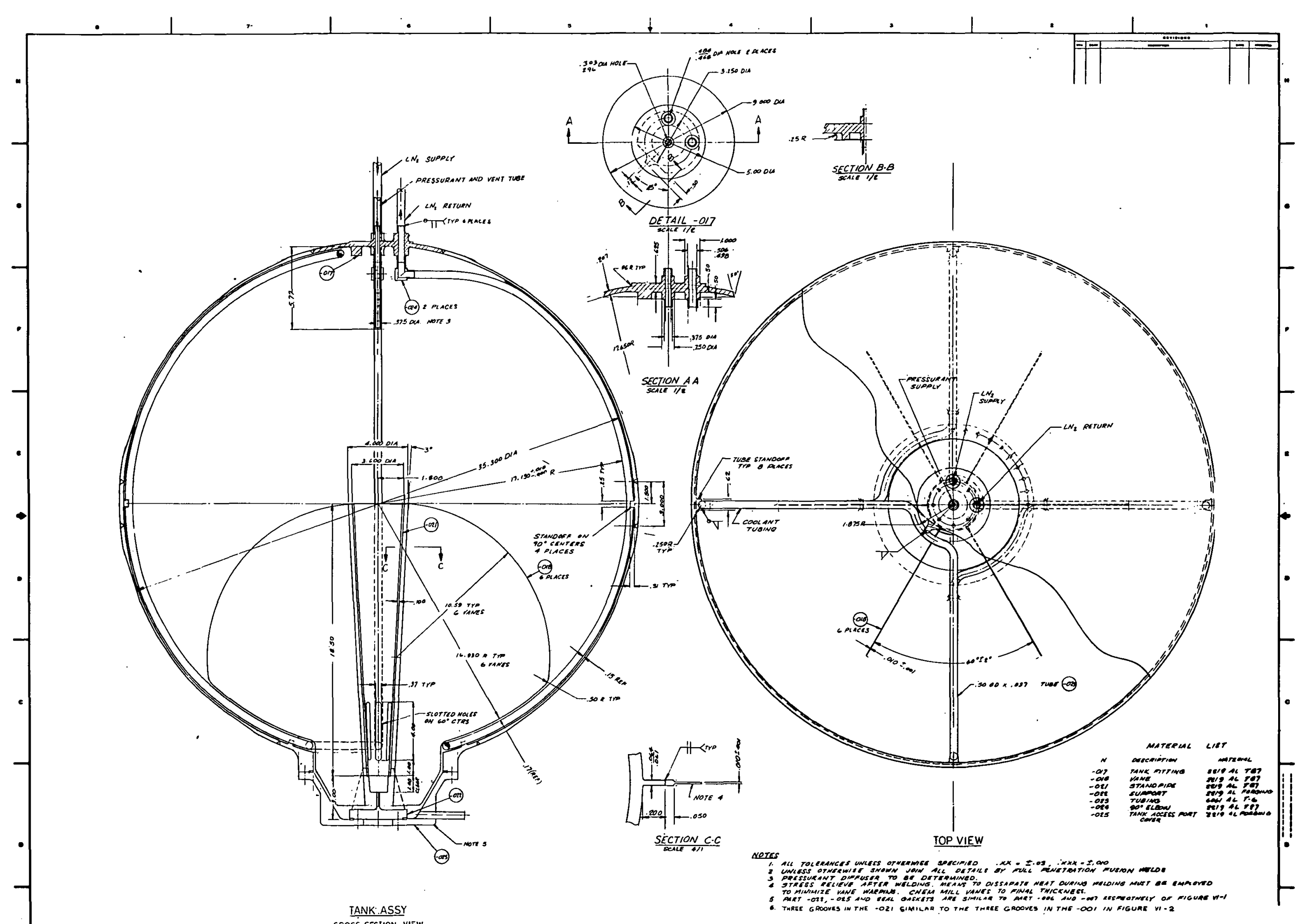




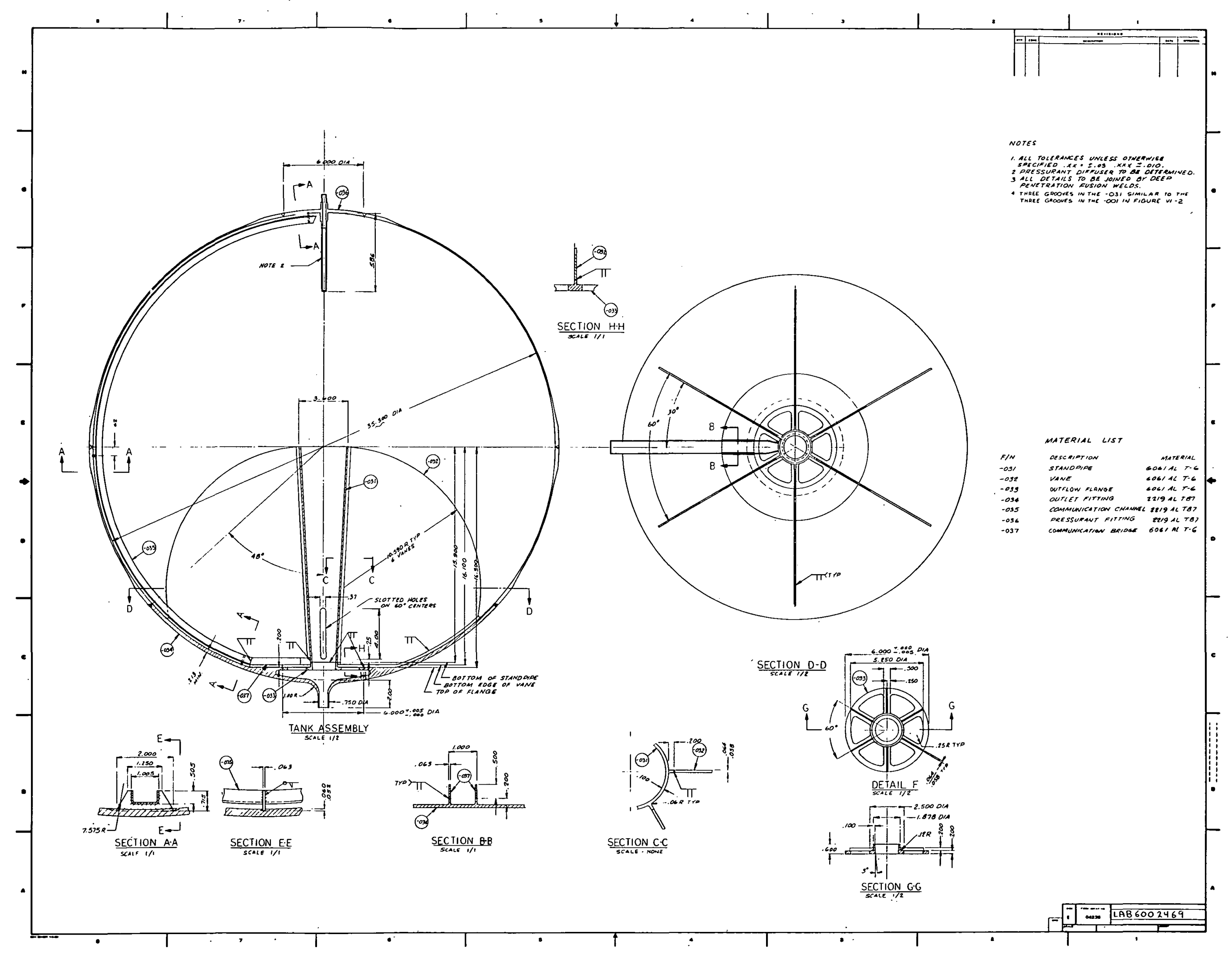

DI-9

Fig. VI-4 Fruhol Low-g Surface Tension Propellant Acquisition System, All-Welded Design for Flox Tank FOLDOUI ERAME 
1. Oxidizer Acquisition System Design - Three specific designs were prepared for the oxidizer system and designated as primary, first alternative, and second alternative designs. All three designs are capable of meeting the mission's structural loading environments. In addition, primary emphasis was given to satisfying the fluorine-based propellant fabrication requirements of maximum system cleanliness with zero contaminant trap area joining processes.

Fabrication, assembly, installation, and cleaning procedures will be discussed in the following paragraphs for each of the three oxidizer designs.

a. Primary Design - Three major objectives were considered in developing the primary design. First, the design was to satisfy a modular installation technique described in Chapter III with minimum influence on the fabrication of the oxidizer tank. Therefore, all fabrication and assembly procedures have been designed to allow for the installation and removal of all acquisition device components through a 9-in.-diameter tank access port. Special tank fabrication requirements to accommodate this installation procedure were kept to a minimum. The second design objective was to integrate a $\mathrm{LN}_{2}$ ground hold cooling coil into the acquisition device. The third objective was to employ only acceptable fluorine-based propellant joining procedures in the design.

1) Material Selection - Based on the spacecraft criteria supplied, the tank material for both the Flox and $L F_{2}$ baseline tanks was 2219-T87 aluminum. Therefore, for the primary design, aluminum alloys were specified for all of the acquisition device components to avoid any dissimilar material problems. Dissimilar material problems, such as $F_{2}$ galvanic corrosion, are discussed in Chapter III.

2) Vane Fabrication and Assembly - To accommodate the modular tank installation procedure, the vanes of the primary design must be rolled into a diameter less than 9 in. However, due to the highly corrosive nature of $F_{2}$ or Flox and the long mission life required, vanes should be as thick as possible. Thus, these considerations, together with the results of the vane rolling structural analysis in Chapter III, require that only 7075-T6 or 2219T87 aluminum be used for the vanes. Based on the previous discussion of fluorine compatible joining processes presented in Chapter III, the vanes can only be attached to the standpipe assemblies by either fusion welding or by a completely sealed mechanical joint. Because a sealed mechanical attachment scheme is unworkable, 
it was decided to fusion weld the vanes to the standpipe. Fusion welding then limits the vane material to 2219-T87 aluminum, because 7075-T6 cannot be welded.

A butt welding technique using an increased vane thickness in the area of the weld is specified for attaching the vanes to the standpipe (see Fig. VI-1). This technique offers the best chance of successfully welding the vanes to the standpipe assembly without vane warping or burnthrough. Thickened vanes in the weld area has been specified for two reasons: (1) they tend to reduce vane burnthrough or warping during the welding process; and (2) the bending point is shifted away from the welded joint to a region on the vane having greater strength. When the vanes are welded to the standpipe, the vane material will be annealed in the area of the weld. Based upon the vane rolling structural analysis presented in Chapter III, a uniformly thick vane will have its maximum bending point occur, during rolling, at its attachment point to the standpipe. Therefore, welding of a uniformly thick vane would reduce the bending capability of the vane because of the anneal involved. By increasing the vane thickness at the attachement point, the region of maximum bending stress shifted from this annealed area to the thinner T87 heat treated material. Based on the vane structural analyses using 2219-T87 aluminum and the standpipe dimensions shown in Fig. VI-1, the vane thickness of the primary design would be approximately 0.009 in. to accomplish modular installation. For an annealed 2219 aluminum, the vane thickness would have to be less than 0.002 in. These values are based on a tank access port diameter of 8.7 in. instead of 9.0 in. to allow for some clearance of a vane rolling tool. The 0.009-in. thickness appears to be a marginal minimum value for ten years' service in fluorine propellants based on present compatibility knowledge. A vane thickness of 0.002 in. is unacceptable; a vane with this thickness could not be welded and would not be compatible with a ten-year service requirement.

To provide the dual thickness vanes needed for the primary design, a chemical milling process is shown. This milling process can be done before or after the vanes are welded to the standpipe assembly. If done after welding, the likelihood of warping or annealing the vanes in the thin bending regions would be reduced. However, if a vane is damaged in the milling process; the entire standpipe assembly would be rejected. If the vanes are milled before welding, vane damage would not require rejection of the standpipe assembly. However, the possibility of vane warping or annealing would be greater. 
Butt welding the vanes to the standpipe assembly would be extremely difficult because the areas to be welded are relatively thin. Proper heat sinks must be provided to avoid warping or annealing of the adjacent areas near the weld. Usually electron beam welding is employed to weld thin components. However, as stated in Chapter III, electron beam welding is not applicable on aluminum alloys due to weld joint cracking. To maintain operational characteristics of the acquisition device, a tolerance of $\pm 2^{\circ}$ was placed on the angle between the vanes. The welding process must be able to maintain this limit.

Other materials were considered to avoid the use of the proposed 2219 aluminum butt weld. One approach would be to use 6061-T6 in place of 2219-T87. The 6061 aluminum is generally considered easier to weld than 2219 (Ref VI-1). Therefore if 6061 was used, the possibility of vane warping would be reduced. Annealing of the thin bending regions of vanes could probably be permitted since the $\mathrm{T} 6$ heat treated condition could be regained by re-heat treating the completed standpipe with vanes assembly after welding. The T87 condition for 2219 aluminum could not be regained after welding because this conditon requires application of a stress load during the heat treating process. The major problem associated with $6061-\mathrm{T} 6$ is that the bending capability of the vanes would be drastically reduced. For the dimensions of the primary design shown in Fig. VI-1, the vane thickness would have to be less than 0.006 in. to accommodate tank installation without plastically deforming. This value is based on the vane rolling structural analysis presented in Chapter III. Vane thicknesses below 0.010 in. are undesirable because of fluorine compatibility considerations. Therefore, although using 6061-T6 would improve the chances of a successful vane weld, vane thicknesses too thin to satisfy mission life requirements would result.

0ther materials that might be used to replace the 2219-T87 aluminum are the 300 series stainless steels. The 300 series of stainless steel is highly compatible with the fluorine-based propellants. These alloys can also be electron beam welded. Therefore, if the material for the primary design were changed to a 300 series stainless steel such as 304L, no vane welding problems would exist. Also, based on the vane rolling structural analysis presented in Chapter III, vane thicknesses can be as high as 0.012 in. and still accommodate tank installation. This value is for a $304 \mathrm{~L} 60 \%$ cold reduced stainless steel using the standpipe dimensions of the primary design shown in Fig. VI-1 with a tank access port diameter of 8.7 in. (rolling tool clearance). The only major problem with using 
$304 \mathrm{~L}$ is that the tank material would also have to be changed to a 300 series stainless steel. A 304L device with a 2219 aluminum tank would not be advisable because of the dissimilar metal problems that would be created. These problems (galvanic corrosion) are discussed in detail in Chapter III. The only 300 series stainless steel that would be applicable as a lightweight propellant tank material would be 301 cryoform. With this material, tank weights lighter than those fabricated out of 2219-T87 aluminum could be attained (Ref VI-2).

3) Standpipe Fabrication and Assembly - One of the major objectives of the primary design was to satisfy modular tank installation with a minimum of special tank fabrication requirements. Based upon the cooling coil integration discussion presented in Chapter III, this objective can only be satisfied for the standpipe-with-vanes concept by incorporating a cooling coil into the body of the standpipe.

Two methods are available for incorporating a cooling provision into the standpipe body. The first would be to install cooling tubes or channels between the vanes, running the length of the standpipe, on the standpipe's outer surface. Coiling a tube or channel inside or outside the standpipe body is impractical because it would interfere with device operation, create contaminant traps, and, for the external coil, interfere with the attachment of the vanes to the standpipe. Inflow and outflow to the channels or tubes could be provided through the base of the standpipe. This cooling coil integration scheme was not selected for the primary design for two basic reasons. First, to attach a channel or tube to the standpipe would require a fusion weld. A sealed mechanical attachment would be another fluorine-compatible joining method. However it would be extremely difficult to use for this application. As discussed previously, fusion welding of thin aluminum members is also difficult. The second, more important reason why this cooling coil integration scheme was not selected for the primary design was that this scheme would interfere with the standpipe's operation. For the cooling channels or tubes to operate properly, these tubes must be connected to provide a continuous $\mathrm{LN}_{2}$ flow path. To provide this path, connecting loops of tube must be run between the cooling channels on both the top and bottom of the standpipe. These connecting links would protrude either into the center of the standpipe, at its top, or above the top of the standpipe, and thus interfere with its proper operation. 
The second method for integrating a $\mathrm{LN}_{2}$ cooling system in the standpipe body would be to incorporate the cooling channels in the walls of the standpipe itself. This method was selected because it does not interfere with the standpipe's operation or the attachment of the vanes to the outer standpipe surface. Figure VI-1 shows the details of the incorporated standpipe/cooling channel design for the primary oxidizer design. The wall of the standpipe is configured as three $\mathrm{LN}_{2}$ flow channels. One channel has been designated the supply channel, while the other two are the return channels. This was done to provide a greater return flow area to account for any $\mathrm{LN}_{2}$ vaporation occurring in the return flow. The dimensions shown for the channels are arbitrary; a complete thermal analysis is necessary before the channels could be accurately sized. To provide inflow and outflow to the $\mathrm{LN}_{2}$ channels, three connecting tubes have been included in the design. These run from the standpipe out through ihe base of the prope1lant tank. To connect the supply $\mathrm{LN}_{2}$ channel to the return channels, a simple manifolding scheme is provided in the top portion of the standpipe's wall. The ribs of the inner standpipe (part -002 of Fig. VI-1) that separate the standpipe's wall into the cooling channels, terminate before reaching the top of the standpipe. Thus, a connection is provided between the supply and return $\mathrm{LN}_{2}$ flow channels.

Only minor impacts were imposed on the design of the primary oxidizer design by incorporating ground hold cooling channels into the walls of the standpipe. These impacts concerned standpipe outflow area, vane thickness, and inner standpipe volume. By designing the walls of the standpipe as cooling channels, a compromise between inner standpipe volume and vane thickness was made. As discussed in Chapter III, the outer diameter of a standpipe critically influences the maximum vane thickness allowable to install the device. Therefore, the inner diameter of the standpipe was arranged so as to not provide too thin a vane for Flox or $\mathrm{F}_{2}$ service. In addition, the inner diameter of the standpipe not only affects inner standpipe volume, but also standpipe outflow area. Incorporating cooling channels into the walls of the standpipe limits the outflow area of the standpipe to its base area only. The cooling channels prevent the use of outflow slots or holes near the base of the standpipe (see Fig. VI-1). A smaller standpipe inner volume is also provided by the primary design because of the cooling channels. 
The specific assembly details for the primary design of the standpipe/cooling channel assembly are shown in Fig. VI-1. A press fit is shown for the assembly of the inner standpipe (part -002) to the outer standpipe (part -001). Because this press fit joint will not be exposed to either Flox or $F_{2}$, no contaminant trap area problems will be present for this type of joint (see Chapter III). Parts -001 and -002 will be machined out of one piece of 2219-T87 aluminum.

Rolling the standpipe vanes to accomplish modular installation requires the use of a vane rolling tool. As discussed in Chapter III, the design of this tool influences what type of joining processes can be used to attach the base of the standpipe to the tank's outflow port assembly or access port cover. Two rolling tool concepts were discussed in Chapter III. The telescoping tool concept did not seem applicable for at least two reasons. First, it appears to be extremely difficult to design the tool with both strength and telescoping ability without becoming too large so as to effectively reduce the tank access port diameter. Second, to provide adequate removal space for the telescoping tool from around the base of the standpipe would prevent the vanes from extending into the outflow port area of the tank (see Fig. VI-1). Extension of the vanes into the tank outflow port provides greater liquid holding capability at low liquid volumes. Therefore, a rigid type of tool, as discussed in Chapter III, was assumed to roll the vanes for tank installation. This tool concept has been proposed for the installation of Viking Orbiter's PMD (Ref VI-3). The type of joining process to be used in attaching the standpipe to the access port cover is limited to a sealed-type mechanical joint.

A crushable gasket bolted joint is used to attach the standpipe/ cooling coil assembly to the tank access port cover (Fig. VI-1). Although this type of joint does not have the reliability of a welded joint with Flox or $\mathrm{F}_{2}$, it is an adequate mechanical joining process for this application.

4) Communication Channel Fabrication and Assembly - The "U" communication channel can be fabricated by a cold forming operation. However, attachment of this channel to the oxidizer propellant tank is difficult. To install and remove the communication channel through the tank's 9-in.-diameter tank access port, mechanical fasteners are required for tank attachment. Due to Flox and $F_{2}$ compatibility considerations, these joints must not have any contaminant trap areas. To satisfy both these criteria, the type of sealable bolt shown in Fig. VI-1 has been proposed to attach the communication channel to its tank. Crushable gaskets are positioned

VI -16 
around the bolt so that, on final torqueing of the bolt, these gaskets will deform and flow, sealing off all contacting surfaces. Both aluminum gaskets (1100-0) and bolts (7075-T6) are specified for the bolting procedure to prevent any gasket loosening due to thermal contractions (bolt, gasket, channel, and tank wall should all shrink at same rate).

The above channel bolting technique is experimental in nature. Available data indicate that this technique has never been experimentally tried with any fluorine-based oxidizer. The reason it has been proposed here is that no other mechanical joining technique seems superior. Therefore, before the use of this bolting technique could be applied to any flight system, extensive experimental testing is necessary.

The communication channel is bolted to the tank at three places: tank access port flange, tank equator, and pressurization port flange. This was considered the minimum number of attachment points necessary to prevent contact between the communication channel and tank wall during spacecraft operation. If the communication channel rubs against the tank wall, a Flox or $F_{2}$ fire could result. To provide the boss areas in the tank for the channel bolting, special provisions will have to be made during tank fabrication. These are the only special tank fabrication requirements imposed by the primary oxidizer design.

5) Miscellaneous Fabrication and Assembly Details - The tank access port cover has a hole at its base (Fig. VI-1). This allows for the passage of a standpipe holding and positioning tool. Once the standpipe assembly and vane rolling tool is inserted into the propellant tank some means must be provided to hold the standpipe assembly in place as the vane rolling tool is removed. Also, due to the relatively small clearance between the vanes and oxidizer tank wall, the tank access port cover will have to be bolted to the tank before bolting the standpipe assembly to the cover. To accomplish this, some means has to be provided to first hold the standpipe assembly in the upper portion of the tank as the tank access port cover is being bolted. Then the standpipe assembly would be lowered and positioned so that it could be bolted to the tank access port cover. Thus, the hole in the tank access port cover provides for the use of a holding and positioning tool. Flox or $\mathrm{F}_{2}$ leakage through this hole should not be a problem because adequate sealing with a gasket can be obtained. 
6) Device Assembly, Tank Installation, and Cleaning Procedures

Device Assembly - The steps of device assembly for the primary oxidizer design are as follows for the communication channe1:

1) Cold form channel;

2) Weld attachment brackets to channe1.

The steps for the standpipe assembly are:

1) Machine or form al1 components;

2) Press fit inner standpipe into outer standpipe;

3) Closure weld cooling channels;

4) Weld $\mathrm{LN}_{2}$ supply and return tubes to standpipe support;

5) Weld standpipe support and $\mathrm{LN}_{2}$ supply and return tubes to standpipe base;

6) Weld vanes to standpipe;

7) Chemically mill vanes to proper thickness (steps 6 and 7 may be reversed).

Device InstaZlation - The device installation steps are as follows:

1) Insert communication channel assembly into tank;

2) Bolt channel to tank wall;

3) Attach standpipe holding and positioning tool and vane rolling tool to standpipe assembly and roll vanes to required installation diameter;

4) Insert standpipe assembly into tank;

5) Unro11 vanes and remove vane rooling tool;

6) Install gaskets in tank access port cover;

7) Bolt tank access port cover to tank;

8) Lower and position standpipe assembly;

$\mathrm{VI}-18$ 
9) Bolt standpipe assembly to tank access port cover;

10) Remove standpipe holding and positioning tool from standpipe assemb 1y.

Quality control checks by X-ray of each weld are essential. Checkout of the structural integrity under simulated launch loads, including slosh and vibration is required. Verification of the structural compatibility with mission temperature extremes is necessary.

Device Cleaning - For any low-g propellant acquisition device, precise cleaning is mandatory for two reasons: assure propellantmaterial compatibility, and prevent degradation (increase) of propellant contact angle. If certain organic contaminants (stearic and oleic acids, paraffin oil, etc) are present in sufficient quantity, propellant contact angles may increase to a point where operation may be seriously compromised. Cleaning procedures must, therefore, be selected so contaminants that degrade propellant or propellant/material compatibility and contaminants that cause an increase in contact angle are minimized or eliminated.

For the primary oxidizer design, or for any propellant acquisition system using fluorine-based oxidizers, the major contaminant concern is one of propellant/material compatibility and not degradation to contact angle. Contaminants that might affect the contact angles of either Flox or $\mathrm{LF}_{2}$, will not survive exposure to either prope1lant.

Therefore, no contaminants, whether they affect contact angle or not, can be tolerated for the primary oxidizer design because reaction with $G F$ during passivation prior to propellant loading could result in destruction of the entire propellant system.

Two types of cleaning procedures are available (Ref IV-4). One procedure uses chemicals, while the other employs a high temperature vacuum (i.e., vacuum annealing). Both of these procedures, if performed properly, can effectively clean components well enough to survive fluorine passivation. However, because the vacuum annealing process does actually anneal metal in its operation, it is not applicable for structural components. Since the majority of the components for the primary oxidizer design must remain in a heat-treated condition (i.e., 7075-T6 bolts, 2219-T87 vanes) to satisfy modular tank installation, vacuum annealing is not recommended for cleaning of the primary oxidizer design. 
The following cleaning steps are recommended for the primary oxidizer design:

1) Before assembly, all parts should be cleaned by a proper chemical cleaning process;

2) The same chemical cleaning process should then follow component assembly. In addition, all joining processes should be performed under strict cleanliness;

3) Before the device is installed, the propellant tank should be cleaned with a proper chemical process;

4) The last cleaning operation (chemical type) should be performed before tank passivation. In addition, a chemical process may be advisable after tank closure and also required after any qualification tests in which the propellant tank is filled with a referee fluid.

Research at Martin Marietta on cleaning process for heat pipe operations indicates that the following chemical cleaning process for aluminum is adequate for the chemical cleaning of the primary oxidizer design ( $\operatorname{Ref}$ IV-5).

1) Vapor Degrease - Trichlorethylene EPS 50046 (Martin Marietta Spec);

2) Alkaline Clean - Turco 4215-6 EPS 50036 (Martin Marietta Spec) ;

3) Demineralized Water Rinse;

4) Acid Deoxidize - Turco Smut Go FPS 50029 Method II (Martin Marietta Spec);

5) Demineralized Water Rinse;

6) Alkaline Etch EPS 50029 Method II, sodium hydroxide, sodium glucomate, sodium sulfide;

7) Demineralized Water Rinse;

8) Acid Deoxidize - Turco Smut Go EPS 50029 Method II;

9) Demineralized Water Rinse;

10) Hot Nitrogen Dry.

VI-20 
Step 6 may not be applicable for the vanes of the primary design because excessive etching could reduce vane thickness too much.

b. First Altemative Design - In the primary oxidizer design, two features are present that may detrimentally influence the design to satisfy a 10-year mission life. These features are the vane thickness and the communication channel attachment method. They result from an attempt to use a modular installation procedure. An alternative design was considered to improve these design features and thereby increase the ability of the system to meet a 10-year mission life. However, the alternative design required a compromise on the use of modular installation.

In the alternative design, the $\mathrm{LN}_{2}$ cooling tubes were mounted on the inner propellant tank wall instead of being incorporated in the body of the standpipe. As discussed previously, incorporation of cooling channels in the standpipe body reduced the vane thickness. Therefore, installation of the cooling tubes on the tank wall instead of in the standpipe allows use of slightly thicker vanes that are more compatible with a 10-year mission requirement.

The placement of the cooling tubes on the tank wa11 also has the advantage, when properly arranged, of providing propellant communication. Thus, a separate communication channel is not required. As far as modular installation was concerned, however, it was found that the cooling tubes could not be installed in the tank through the access port. Therefore, the cooling tubes must be included as part of the tank assembly procedure. The tubes would be attached to the tank wall by welding. Therefore, although modular installation of the cooling tubes could not be accomplished, the alternative design does eliminate mechanical fastening of the communication channel as required in the primary design. Elimination of mechanical fasteners is desirable for the 10-year mission requirement.

In regard to modular installation, the propellant acquisition device can still use this assembly procedure.

1). Material Selection - Based upon the same considerations presented under the primary design, aluminum alloys are specified for all of the first alternative design components.

2) Vane Fabrication and Assembly - The same comments presented for the primary design also apply, in general, to the fabrication and attachment of the vanes for the first alternative design. 
The same 2219 aluminum butt weld is recommended (Fig. VI-3). However, vane thicknesses $0.001 \mathrm{in}$. greater than the primary design are shown becuase the outer diameter of the standpipe is now less than in the primary design since no cooling coil is incorporated in the standpipe walls.

3) Standpipe Fabrication and Assembly - The standpipe shown in Fig. VI-3 is easier to construct than the primary design standpipe and also provides greater outflow area and inner standpipe volume. These advantages occur because no cooling provision is incorporated into the standpipe. For manufacture of the standpipe, machining out of one piece of 2219 aluminum seems advisable.

Based on the same reasons presented under the primary design, the same sealed type of mechanical joint is shown for the attachment of the standpipe to the tank access port cover.

4) Communication Channel Fabrication and Assemb1y - The $\mathrm{LN}_{2}$ cooling coil in the first alternative design provides four communication channels (Fig. VI-3). The cooling coil is welded to the tank at the tank access port, tank equator, and pressurization flange. Tabs are provided at these locations for attachment. The coil is constructed of 6061-T6 tubing and connected together to form one continuous loop. Inflow and outflow to this tube are provided through the pressurization flange. To install and weld the channels or cooling tube into the tank requires that this tube be installed during tank fabrication. Precise tank fabrication procedures incorporating the attachment of the cooling coil are listed in the next subsection.

Welding the cooling coil to the tank tabs presents a problem. If the walls of the tubing are too thin, burnthrough may result during welding. Therefore, the thickness of tube wall should correspond to that of the attachment tab so that this condition will not occur. Under this condition, the tube wall and the tab will uniformly heat and melt at the same rate.

5) Assembly, Installation, and Cleaning Procedures

Device and Tonk Assembly - The steps of device assembly for the first alternative oxidizer design are as follows:

1) Machine and form all components;

2) Weld standpipe support to standpipe;

VI-22 
3) Weld vanes to standpipe;

4) Chemically mill vanes to proper thickness (steps 3 and 4 may be reversed).

The tank assembly steps are:

1) Weld tank girth ring to bottom spherical shell of tank;

2) Form cooling tube/communication channel assembly (part -023 of Fig. VI-3);

3) Weld $90^{\circ}$ elbow to pressurant/cooling coil tank fitting (part -024 to -017 . of Fig. VI-3);

4) Position cooling tube/communication channel assembly in bottom spherical shell of tank;

5) Weld cooling tube/communication channel assembly to tank girth ring and bottom shell of tank;

6) Weld pressurant/cooling coil tank fitting to cooling tube/ communication channel assembly;

7) Position top shell of tank on tank girth ring and pressurant/ cooling coil tank fitting;

8) Perform closure welds.

Device Instalzation - Same as primary design minus communication channel installation.

Device Cleaning - The same comments and procedures discussed for the primary design also apply to the first alternative design.

c. Second Altemative Design - The primary objective of the second alternative was to present as simple a design as possible using only joining procedures that give maximum compatibility with either $\mathrm{LF}_{2}$ or Flox. All joining operations for this design were limited to welding. For this reason, only the third or integral tank installation procedure defined in Chapter III was considered for assembly. Figure VI-4 shows the design. Ground cooling is provided for this design by an external system described in Section C. Based on the simplicity and greater propellant compatibility, the second alternative design is preferred for the oxidizer system. 
1) Material Selection - Aluminum alloys were specified for the second alternative design for the same reasons given for both primary and first alternative designs.

2) Vane Fabrication and Assembly - Vanes for the "second alternative design will be fabricated from 6061-T6 aluminum. This alloy was selected because it is easily weldable and high strength is not required. The vanes for the second alternative design do not need to be rolled for tank installation. A vane thickness of approximately $0.061 \mathrm{in}$. was specified. This value was obtained as a compromise between device weight, propellant compatibility, and welding considerations.

To weld the vanes to the standpipe and outlet fitting (see Fig. VI-4) a butt weld is preferred because it offers the best chance of a successful weld. Unlike the other two oxidizer designs, the second alternative does not require chemical milling because the vanes have uniform thickness. Therefore, 0.061-in. 6061-T6 rolled stock can be used for the vanes.

3) Standpipe Fabrication and Assembly - For the standpipe of the second alternative design, machining of a solid piece of 6061-T6 aluminum is the most desirable procedure. 6061-T6 aluminum was chosen because it is easily weldable and high strength was not required.

4) Communication Channel Fabrication and Assembly - Fabrication and attachment of the U-shaped communication channel (shown in Fig. VI-4) to the tank can easily be accomplished. Tabs are provided on the tank wall for attachment. Three attachment points have been proposed to rigidly hold the channel in place. A twopart structure called a communication bridge is proposed to connect the standpipe base and the communication channel. 2219-T87 aluminum is used for the communication channel because a structural alloy is required for the cold forming of the channel into its proper shape.

\section{5) Assembly and Cleaning Procedures}

Device/Tank Assembly - The steps of device/tank assembly for the second alternative oxidizer design are as follows:

1) Machine or form all components;

2) Weld standpipe to outflow flange;

3) Weld outflow flange to outlet fitting; 
4) Weld communication bridge to outlet fitting and standpipe;

5) Weld vanes to outlet fitting and standpipe;

6) Weld communication channel to outlet fitting and communication bridge;

7) Weld outlet fitting to bottom spherical shell of tank;

8) Weld communication channel to bottom tank she11;

9) Weld pressurant fitting to communication channel;

10) Closure weld top tank shell to pressurant fitting and bottom tank she11.

Cleaning Procedures - In general, the same comments presented for the primary design also apply to the second alternative design. However, since none of the components of the second alternative design are structural members, except for the tank shells and the outlet fitting, a vacuum annealing cleaning procedure can be used for some of the parts. One nonstructural part for which vacuum annealing would not be suitable is the vanes. Vacuum annealing may warp the vanes.

2. Fue1 Acquisition System Design

Figure VI-2 presents the fuel acquisition system design. It is similar to the primary oxidizer acquisition system design, except for the ground hold cooling provision, which is not required by the fuel. Like the oxidizer designs, the fuel system design is capable of meeting the missions's structural loading environments. In addition, there was emphasis on only using fabrication and joining processes that would provide maximum mission life. For this reason, many of the joining processes used on the oxidizer designs are also applied to the fuel design.

The objective of the fuel design, like that of the primary oxidizer design, was to satisfy modular installation with minimum. influence on the fabrication of the fuel tank. To accomplish this, sealed-type mechanical joints similar to those used in the primary oxidizer design were also used on the fuel system. These types of joints are not as reliable as welded or brazed joints for exposure to Flox or $\mathrm{LF}_{2}$. However, since $\mathrm{N}_{2} \mathrm{H}_{4}$ and $\mathrm{MMH}$ are not as difficult to handle as the fluorine-based oxidizers, these joints will provide better reliability for a 10-year mission. 
For this reason alternative designs are not presented for the fuel design. However, the type of all-welded design shown in Fig. VI-4 for the second alternative oxidizer system could also be applied to the fuel acquisition system if increased mission life reliability is necessary.

Fabrication, assembly, device installation, and cleaning procedures are discussed in the following paragraphs for the fuel design.

a. Material Selection - Based upon $\mathrm{MMH}$ and $\mathrm{N}_{2} \mathrm{H}_{4}$ compatibility considerations, only aluminum and titanium alloys are applicable materials to construct the fuel design (Ref VI-6).

b. Vane Fabrication and Assembly - To accommodate modular insta1lation, the vanes of the fuel design must roll for tank insertion. Therefore, $6 \mathrm{Al}-4 \mathrm{~V}$ titanium was selected for the vane material because it has excellent bending strength (see Chapter III) and is compatible with either $\mathrm{MMH}$ or $\mathrm{N}_{2} \mathrm{H}_{4}$.

To attach the vanes to the standpipe, a 3003 A. brazing technique is specified. As discussed in Chapter III two fuel-compatible brazing techniques are available for titanium $(48 \mathrm{Zr}-4 \mathrm{Be} \mathrm{TI}$ and $3003 \mathrm{Al}$ ). The 3003 process was selected because its brazing temperature is lower than that for the $48 \mathrm{Zr}-4 \mathrm{Be} \mathrm{TI}$ process (Ref VI-7). With lower brazing temperatures, the chances for vane warping are reduced. Brazing was selected over welding because of its simplicity. To braze the vanes to the standpipe an increased vane thickness in the area of the braze joint is necessary to shift the maximum bending point of the vanes away from the brazed joint because this joint is weaker than the vane itself (see primary oxidizer discussion). Therefore, due to the dual thickness of the vanes, chenical milling is required to reduce the vanes to their final thickness. This milling should be performed after brazing to reduce the possibility of vane warping. A $\pm 2^{\circ}$ tolerance has also been placed on the angle between vanes based upon operational considerations. The brazing process must maintain this tolerance.

The thickness of the vanes shown in Fig. VI-2 was obtained as a result of a compromise between various factors. Based on the structural vane rolling analysis of Chapter III, the thickness of the $6 \mathrm{Al}-4 \mathrm{~V}$ titanium vanes for the fuel design can be as great as $0.016 \mathrm{in.}$ and still satisfy installation. However, the force required to roll $0.016-i n$. vanes would be large. Vanes in the fuel design will not be exposed to the corrosive environment of

VI-26 
the oxidizer system, so vane thickness does not have to be as great to satisfy the 10 -year mission requirement. Therefore, vane thickness was reduced to $0.011 \mathrm{in}$. to reduce the force needed to roll the vanes for tank installation. Since this value gives greater bending capability than needed, tolerances on vane thickness can also be greater than for the oxidizer designs. A $\pm 0.002-$ in. tolerance has been placed on vane thickness for the fuel design.

c. Standpipe Fabrication and Assembly-With $6 \mathrm{Al}-4 \mathrm{~V}$ Ti vanes, the material for both the standpipe and its support hub is also $6 \mathrm{Al}-4 \mathrm{~V} \mathrm{Ti}$ to allow the use of $3003 \mathrm{Al}$ brazing for vane attachment. Brazing is also specified for the joining process to attach the stand-pipe to its support base because brazing is easier to perform than welding.

To attach the standpipe assembly to the tank's access port, the same type of mechanical joint used for both the primary and first alternative oxidizer designs is shown for the fuel design. This joint is specified becuase of the same vane rolling tool considerations associated with the primary and first alternative oxidizer designs. Although this type of joint is not as reliable as either a brazed or welded joint, it should withstand 10 years of exposure in either MMH or $\mathrm{N}_{2} \mathrm{H}_{4}$. Also, the tank access port cover design is the same as the primary and first alternative oxidizer design based on the same reasons.

d.' Communication Channel Fabrication and Assembly - The same type of communication channel attachment procedure proposed for the primary oxidizer system design is also proposed for the fuel system. This procedure provides the best compromise between long-life propellant compatibility and modular tank installation. However, unlike the primary oxidizer design, the sealed type bolting technique seems to have a higher reliability for the 10-year mission. Thermal contraction problems associated with cryogenics will not be present in the fuel system. The bolts are 7075-T6 aluminum to prevent any galling during attachment because no bolt lubricants will be used. An all-metal system is desired for maximum compatibility.

\section{e. Device Assembly, Tank Installation, and Cleaning Procedures}

Device Assembly - The steps of device assembly for the fuel design communication channel are the same as for the primary oxidizer design. The standpipe assembly steps are: 
1) Machine or form all components;

2) Braze vanes and standpipe support to standpipe;

3) Chemically mill vanes to proper thickness. .

Device Installation - Same as primary oxidizer design.

Cleaning Procedures - Unlike the oxidizer designs, cleaning concerns for the fuel design include degradation of propellant contact angle and propellant-material compatibility. Unlike the fluorine-based propellants, contaminants that affect contact angle will not be reacted away when exposed to either $\mathrm{MMH}$ or $\mathrm{N}_{2} \mathrm{H}_{4}$. In addition, cleaning processes that eliminate contaminants which affect propellant material compatibility (MMH and $\mathrm{N}_{2} \mathrm{H}_{4}$ decomposition) may not remove contaminants that affect propellant contact angle. The opposite is also true. Therefore, cleaning procedures that remove both types of contaminants are mandatory for the fuel design.

Three types of cleaning procedures are available for the fuel design. Two are chemical and the third is a vacuum annealing process. One chemical type is an acid-pickling process and the other is an alkaline process. Based on a previous study (Ref VI-4), the acid cleaning techniques are only effective against propellant-material compatibility contaminants such as oxide coating that can cause $\mathrm{MMH}$ or $\mathrm{N}_{2} \mathrm{H}_{4}$ decomposition. The acidtype cleaners are not effective against contact angle contaminants. The opposite situation is present for the alkaline techniques. Therefore, both chemical types of cleaning are necessary for total cleaning. Vacuum annealing removes both contact angle contaminants and propellant compatibility contaminants, but has the disadvantage of annealing any metal component in its operation.

Based on the above discussion, the following cleaning steps are recommended for the fuel design:

1) Before assembling the device all parts (except the vanes and the 7075-T6 bolts) should be cleaned by vacuum annealing. Because the 7075 aluminum bolts must remain in the T6 condition and the vanes may warp, vacuum annealing is not applicable for those items. The vanes and bolts will undergo an acid cleaning (care must be taken to not etch the vanes or bolts severely). 
2) After device assembly, all components should be cleaned with and acid cleaning process followed by an alkaline one. In addition, all joining operations should be performed under strict clean room conditions (vacuum annealing cannot be done because degradation of braze joints will occur).

3) Prior to device installation, the propellant tank itself should be cleaned with an acid cleaning process followed by an alkaline process.

4) The last cleaning operation (alkaline only, oxides should not be present) should be performed before propellant tank loading. In addition, an alkaline cleaning process may be: advisable after tank closure and also after any qualification tests requiring the propellant tank to be filled with reference fluids.

Based on a previous study (Ref VI-4), the following acid cleaning procedure would seem to be suitable for the fuel design.

1) Solvent degrease with acetone;

2) Acid pickle with $\mathrm{HNO}_{3} / \mathrm{HF}$ solution;

3) Distilled $\mathrm{H}_{2} \mathrm{O}$ rinse (check pH);

4) Hot nitrogen dry.

Based on cleaning studies for the Viking Orbiter, the following alkaline cleaning procedure is suitable for the fuel design (Ref VI-8):

1) Solvent degrease with acetone $/ \mathrm{H}_{2} \mathrm{O}$ solution;

2) Alkaline clean with Turco 4215;

3) Deionized water rinse, checking $\mathrm{pH}$;

4) Isopropyl alcohol rinse;

5) $\mathrm{GN}_{2}$ dry. 
In designing the system, emphasis was placed on providing gas-free liquid expulsion, because this is the primary function of the propellant acquisition device. Next in importance were centerof-gravity control and the ability to vent and pressurize. The analyses conducted to develop and support the system design are presented in this section. Selection of the configuration of the vanes and the standpipe are included and the outlet, communication channel, and pressurization/vent port are discussed.

1. Vane Profile

Because of the difference in initial ullage, the profile of the acquisition system vanes was not the same for the oxidizer and fuel tanks. The selection of the vane profile for each tank is discussed in the following paragraphs.

a. Oxidizer Tonk - At the nominal propellant temperature, the ullage volume in the oxidizer tank is $20 \%$ of the tank volume. When the maximum propellant temperature is considered, however, the ullage volume would be only $14 \%$. Based on the curve in Fig. III-8 (Chapter III), the height of the device for this volume should be at least $0.96 \mathrm{R}$. The effect of an axial acceleration acting on the tank must also be considered in selecting the height of the device. The shape of the $14 \%$ volume ullage bubble under the effect of the acceleration, a, acting on the liquid is shown in Fig. VI-5. In this case, the largest purely axial acceleration of $3 \times 10^{-7} \mathrm{~g}$, which may be acting on the spacecraft was used. Under the effect of this acceleration, the ullage bubble is flattened somewhat, so the distance from the bottom of the tank to the ullage bubble is $0.98 \mathrm{R}$.

A height of $1.0 \mathrm{R}$ was selected for the device. With this height the device will be in contact with the ullage bubble at the minimum ullage volume and with an acceleration tending to move the bubble away from the device. At normal temperature and zero $\mathrm{g}$, the bubble will be forced into the device, which will keep the bubble well centered. An overly tall device is not desirable because it compromises the pumping capability of the vane profile and the positioning of the smaller liquid volumes. 


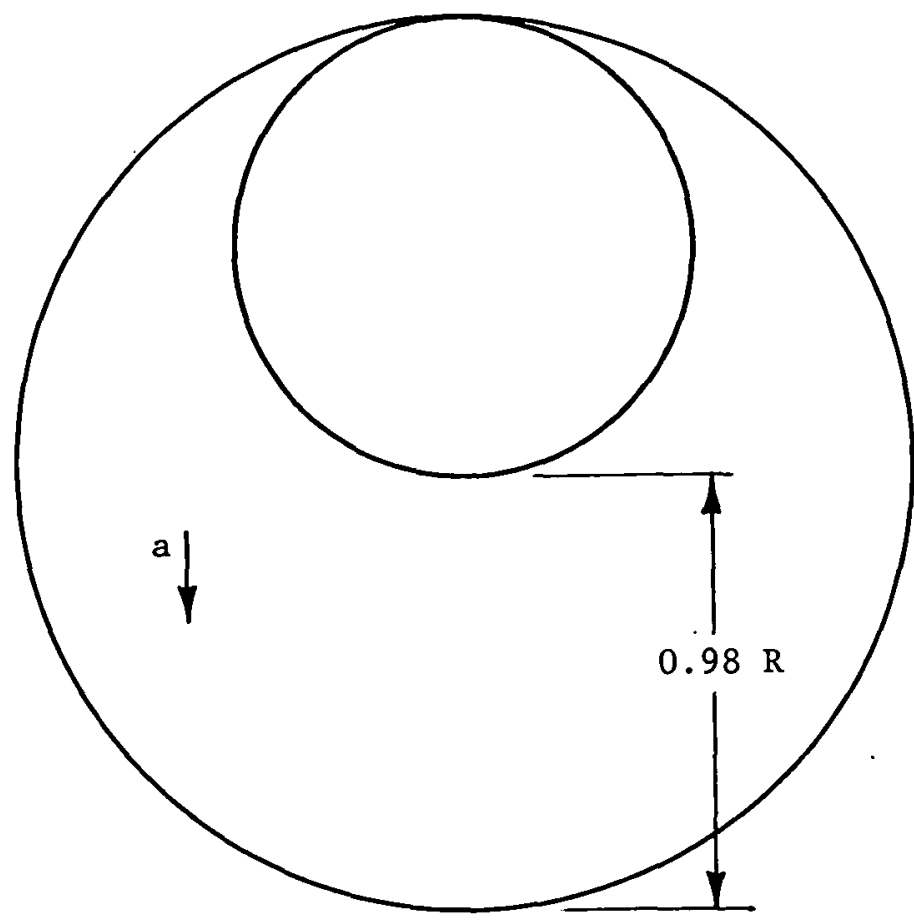

Fig. VI-5 Interface in Oxidizer Tank

With a minimum ullage volume of $14 \%$, a unique location for the bubble would be obtained by just a simple post with the height of 1.0R. There is no inner profile limit that must be exceeded. In this case, a wide, generous vane profile was selected as shown by the simplified drawing of the standpipe and vanes in Fig. VI-6. All dimensions are referenced to the tank radius, $R$. The bubble breakup limit, discussed in the Chapter III analysis of the vane devices, is shown superimposed on the vane profile in the figure. As can be seen, the profile extends across the limit. Bubble breakup will not occur due to the lateral stability of the device, and much better positioning of low liquid volumes is obtained with the selected profile.

Breakup of the ullage bubble would most likely occur at the minimum ullage volume. Under the effect of the maximum lateral acceleration during boom deployment, the bubble would be displaced to the side of the tank. However, only a portion of the bubble would be within the region where the vanes exceed the breakup limit. As the system returns to zero g, liquid will fill between the vanes and the wa11, but not far enough to split the ullage bubble. Therefore, the bubble will be pushed out of the vanes to return to its centered position. 


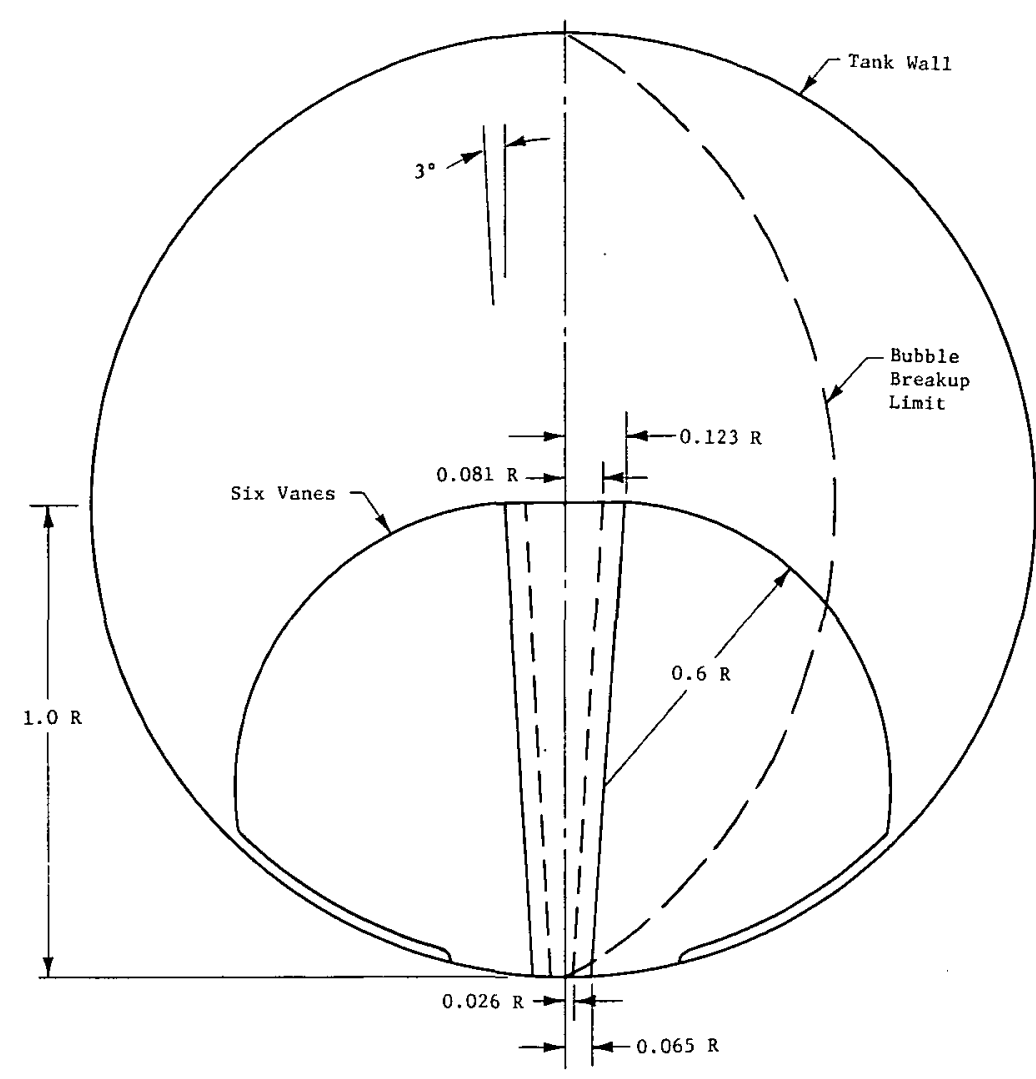

Fig. VI-6 Standpipe with Vanes for Oxidizer Tank

At low liquid volumes, the selected vane profile has a definite advantage over a profile that stays within the bubble breakup limit. With the vane profile close to the wall, liquid will fill the region surrounding the vanes and the wall at the tank outlet. In comparison, a vane that stays within the limit would tend to hold more liquid up within the vanes, leaving less liquid at the outlet.

A device with six vanes was selected. Here again, the emphasis was placed on gas-free liquid expulsion. Six is the minimum number of vanes that will, in conjunction with the profile of the vanes, provide adequate pumping capability. Liquid will orient closer to the tank outlet with six vanes, as opposed to a larger number.

This selected vane system will always be capable of reorienting the liquid so that the center of gravity falls on the centerline of the tank following any disturbance. The ullage bubble will be centered so that venting or pressurization can be accomplished at any time during the mission, with one possible exception. During boom deployment, the magnitude and duration of the disturbance is 
sufficient to displace the ullage bubble away from the pressurization/vent port. Following boom deployment, the bubble will return to the centered position. For the $45-\mathrm{sec}$ period of the deployment, however, venting or pressurization could only occur in a degraded mode because liquid would probably surround the pressurization/ vent tube. Disturbances due to the attitude control system were shown to be slight due to the short duration and relatively long period of the thruster firing. Allowing the ullage to be displaced for a 45-sec period is a small compromise in comparison to the gross overdesign of the device that would be required to keep the bubble centered during boom deployment. Such a design would seriously degrade the reliability of gas-free outflow at the smaller liquid volumes.

b. Fuel Tank - Based on the maximum fuel temperature, the initial ullage volume could be as small as $3.5 \%$ of the tank volume, in comparison to the $5 \%$ ullage at the nominal temperature. The device should have a height of at least $1.35 \mathrm{R}$ for a $3.5 \%$ ullage bubble, as shown by Fig. III-8. Under the effect of the maximum axial acceleration, the bubble is not significantly flattened, as shown in Fig. VI-7. A height of $1.4 \mathrm{R}$ was selected for the device so as to bias the centering of the bubble.

The approach in designing the vane profile for the fuel tank was the same as that used for the oxidizer tank. Since the ullage volume is less than $12.5 \%$, and inner profile limit must also be considered in designing the profile of the fuel tank device so that the centered position will be the only stable position for the bubble. Both the inner and outer limits are shown superimposed on the vane profile in Fig. VI-8. Near the tank equator, the vane profile exceeds the bubble breakup limit. The vane profile has sufficient pumping capability to keep the bubble from being completely displaced into the region where the vanes exceed the limit, however. For this reason, breakup of the ullage bubble should not occur during the mission.

Again, six vanes were selected for the device. Except for boom deployment, the device should maintain the liquid cg on the tank centerline so that venting or pressurization can be accomplished at any time during the mission. The operation and performance of the fuel tank device is similar to the oxidizer tank device. At low liquid volumes, the greater height of the fuel tank device will cause more liquid to be held up within the vanes in comparison to the oxidizer system. This should not present a problem with gasfree liquid outflow, however. 

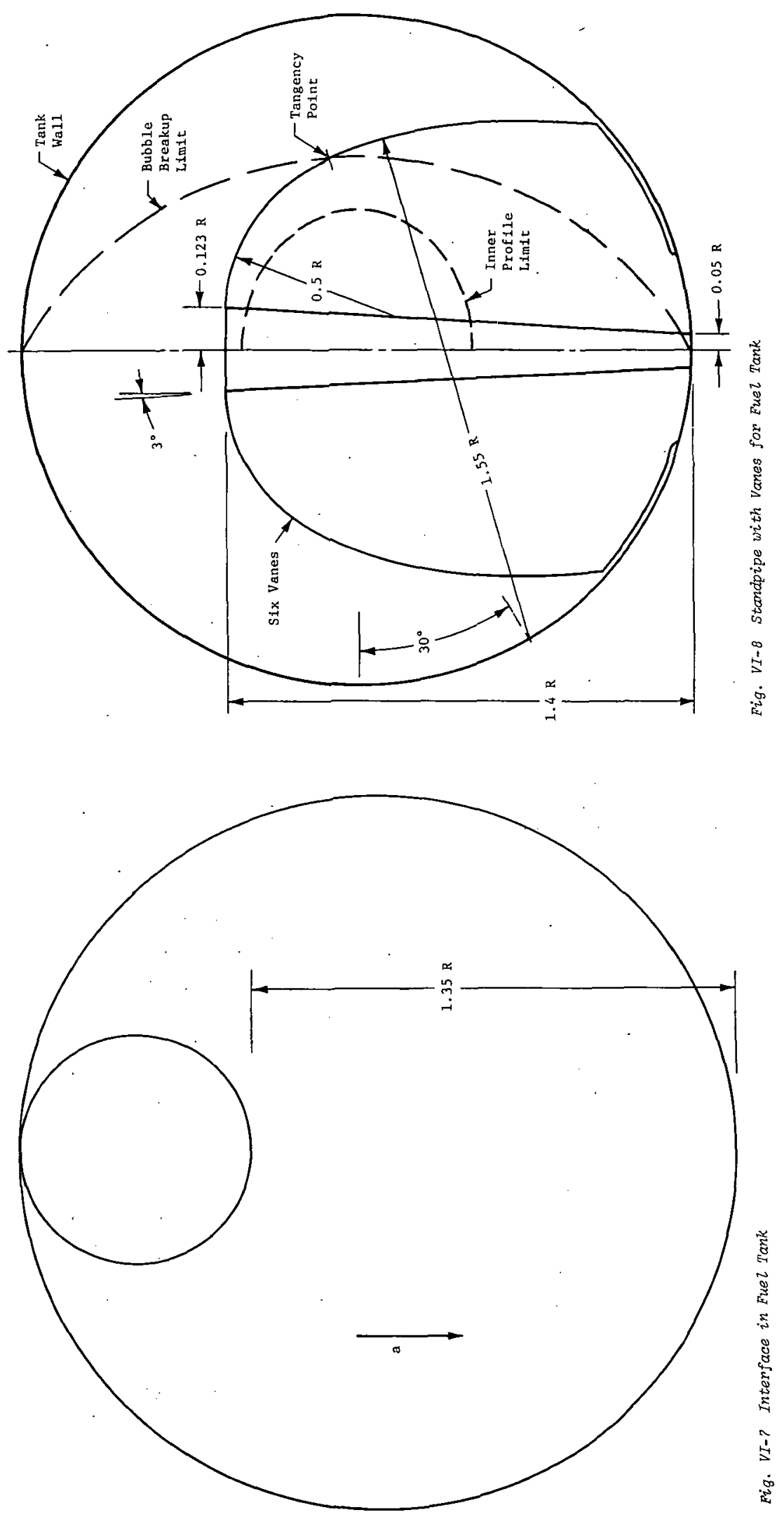

VI-34 
2. Standpipe

The standpipe is a highly stable liquid reservoir with an outlet directly over the tank outlet. Feed of liquid from inside the standpipe to the tank outlet is in parallel with the feed of liquid from outside the standpipe. Liquid does not flow through the standpipe as it leaves the tank. The design of the standpipe for the oxidizer and fuel tanks is discussed in this subsection.

a. Oxidizer. Tonk - In the primary acquisition system design for the oxidizer tank, the ground hold cooling system is incorporated into the standpipe. The internal volume of the standpipe is $0.24 \%$ of the tank volume. This is a rather small volume, but the requirements of modular installation and an integrated ground hold cooling system do not allow a larger standpipe. Regardless, only enough liquid is required to supplement the liquid from outside the standpipe during the start of the spacecraft engine. The tradeoffs in the design of the standpipe will be considered as the design approach is discussed.

The possible range of standpipe volumes is plotted in Fig. VI-9. Taper, $\theta$, and the radius of the base, $r_{b}$, are the variables that define the size of the standpipe. The height is held constant at 1.0R. Shown with a dashed line is the extreme limit to the size of the standpipe. It is the point at which the top of the standpipe has a diameter of 9 in., modular installation through a 9-in. access port would not be possible with a larger stand-. pipe. This is not a realistic limit because the vanes have not been considered. Rolling of the vanes for modular installation depends on the vane thickness, diameter of the standpipe, and the material. Details of the interaction are discussed under the fabrication of the device, but a more typical limit to the standpipe dimeter would be 5 in. With that limit, the possible range of standpipe volumes is less than $1 \%$ of tank volume.

The most attractive approach to modular installation of the device and cooling coils is to incorporate the cooling system into the standpipe. This approach makes use of the vanes as heat exchanger fins and minimizes the number of tank penetrations. The disadvantage of this approach is that the standpipe volume must be decreased. In the alternative designs for the oxidizer tank, the ground hold cooling system was not incorporated into the standpipe. This allowed increasing the volume of the standpipe to $0.45 \%$ of the tank volume. 


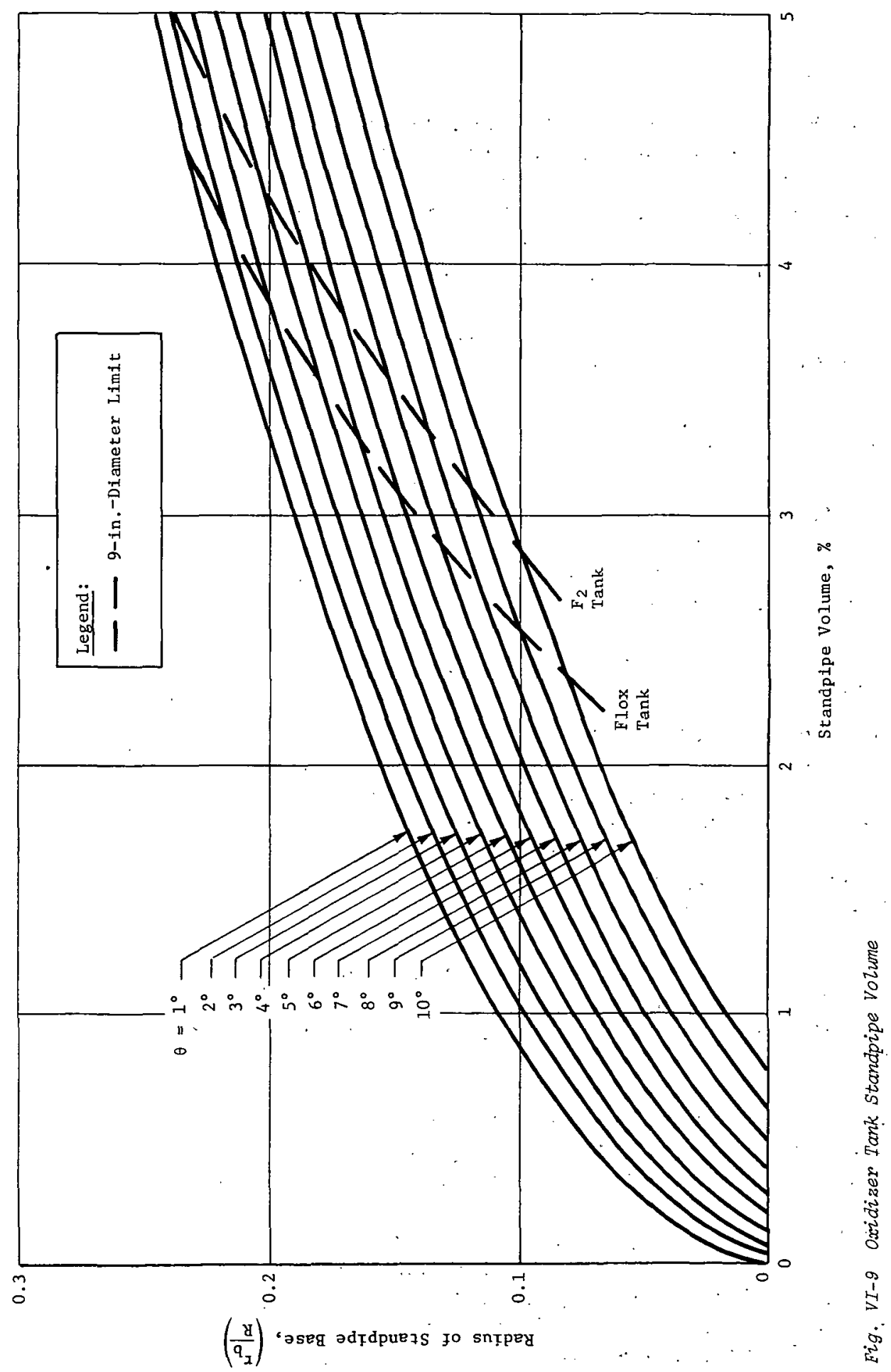


A standpipe having a $3^{\circ}$ taper was chosen for a number of reasons. First of a11, considering only liquid located outside the standpipe, a tapered post or standpipe has more stability than an untapered device. This improves the contribution of the standpipe to the overall stability of the device. The Chapter III analysis shows that the taper improves the positioning of the 1iquid over the outlet. Next, consider liquid located inside the standpipe. With an untapered standpipe, the stability of the interface is independent of the volume of liquid inside the standpipe, assuming the interface is not stuck and does not contact any other surface. As the volume of liquid inside a tapered standpipe decreases, the stability increases. Therefore, as liquid is lost from the standpipe, it becomes increasingly difficult to lose additional liquid.

It is possible to have a portion of liquid separate from the main propellant mass and become positioned inside the standpipe. Slush due to settling is a possible means for this occurrence. In an untapered standpipe, the curvature at each end of the separated portion of liquid would be the same, so there would be no forces acting to return the separated liquid to the bulk propellant. With a tapered standpipe, an unbalance in capillary forces exists across a separated portion of liquid that acts to pump the liquid towards the base of the standpipe. A sketch of the two possibilities is shown in Fig. VI-10. The liquid orientation in the untapered standpipe is stable while that in the tapered standpipe is unstable.

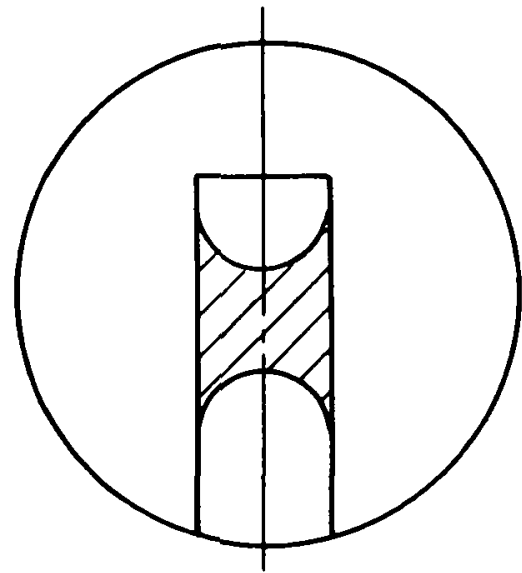

(a) Untapered Standpipe

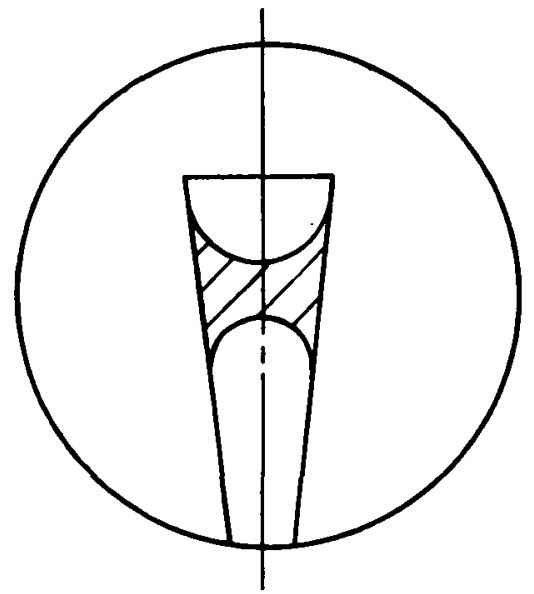

(b) Tapered Standpipe

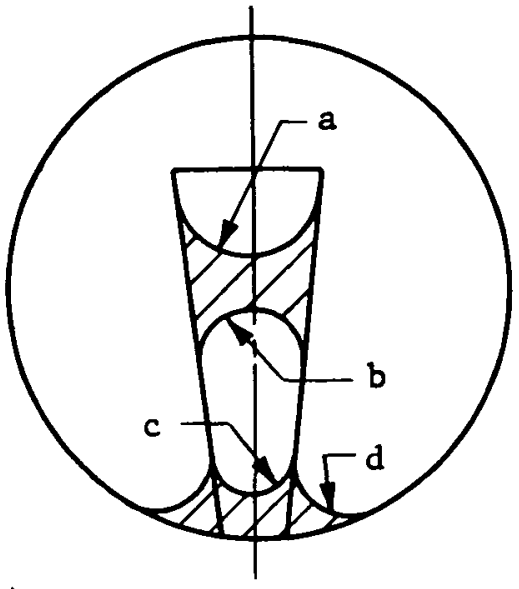

(c) Tapered Standpipe with Bubble

Fig. VI-10 Interfaces within Standpipe 
The situation shown in Fig. VI-10(b) could be further complicated by having some liquid located at the base of the standpipe, thus forming a bubble within the standpipe. In this case clearing of the gas from the standpipe will still occur if the interfaces on each side of the bubble [interfaces $b$ and $c$ in Fig. VI-10(c)] are in contact. When the two portions of liquid are not in contact, equilibrium can be established and the bubble will.remain in the standpipe. This occurs when interfaces a and $d$ in Fig. VI-10(c) both have the some curvature. For this reason, grooves were added to the inside wall of the standpipe. The grooves act as communication channels so the two portions of liquid will always be in contact and purging of gas will take place.

Only at total liquid volumes less than about $3 \%$ of the tank volume will the standpipe become partially empty. The standpipe will fill until the curvature of the interface inside the standpipe is equal to the curvature of the interface around the vanes outside the standpipe. Curvature of the interface inside the standpipe versus the volume of liquid inside the standpipe (expressed as a percentage of the tank volume) is presented in Fig. VI-11. The flexure point on the curve is the point at which the interface becomes stuck at the top of the standpipe. When the standpipe is completely full the curvature is zero. In comparison, the approximate curvature of the liquid within the vanes is plotted versus the volume of liquid outside the standpipe in Fig. VI-12. For volumes less than $5 \%$, the curvature becomes dependent on the configuration of the tank outlet, making the determination of the curvature a complex, three-dimensional problem. At volumes of about $3 \%$ and above, equalizing the curvatures requires that the interface inside the standpipe be stuck at the top of the device.

When the spacecraft engine starts, the standpipe performs its most important function. As the spacecraft begins to accelerate, the hydrostatic pressure due to the difference in height between the liquid inside the standpipe and a lower liquid level outside the standpipe will force the liquid out of the standpipe. If the level of the liquid outside the standpipe is greater than the height of the liquid inside the standpipe, the liquid inside will tend to remain there and the standpipe has little influence on the flow of liquid from the tank.

Resisting the flow of liquid from the standpipe are the viscous forces due to the orifice at the base of the standpipe. The standpipe is only open at the top and the bottom. In the alternative designs, the absence of the ground hold cooling system allows additional openings to be added to the sides of the standpipe. The openings are stable in low ga, so this standpipe would fill the same as the one with solid walls. During outflow, the additional openings would become unstable, providing additional flow area and less viscous resistance so that the standpipe would be emptied at a faster rate.

$V I-38$ 
The contribution of the standpipe is most important during an engine start at low liquid volumes. While liquid outside the standpipe is being settled and collected over the outlet, the volume of liquid inside the standpipe is available to maintain the flow of liquid to the engine. The possibility of suction dip occurring is reduced by the standpipe.

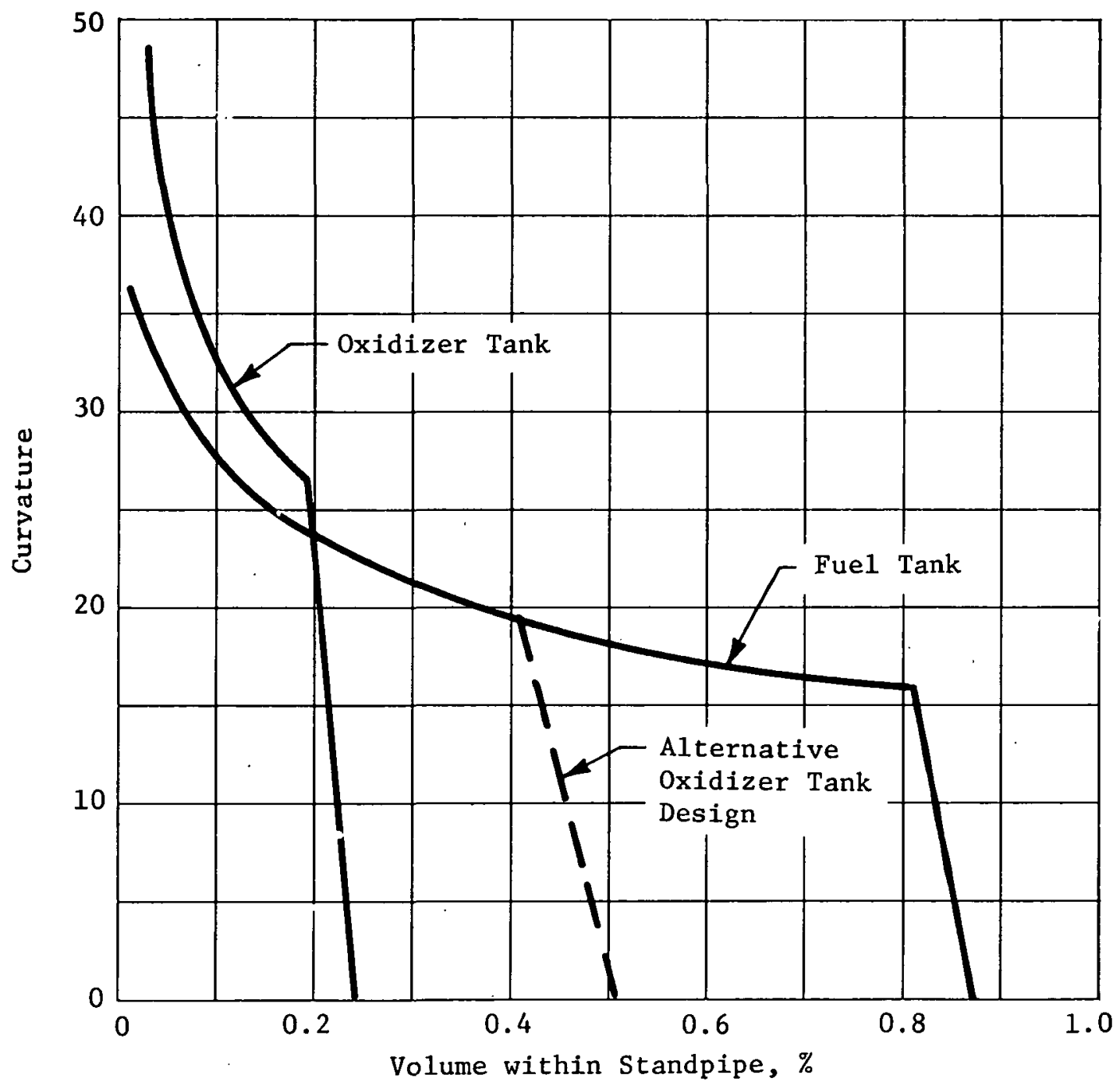

Fig. VI-11 Curvature vs Liquid Volume for Standpipe 


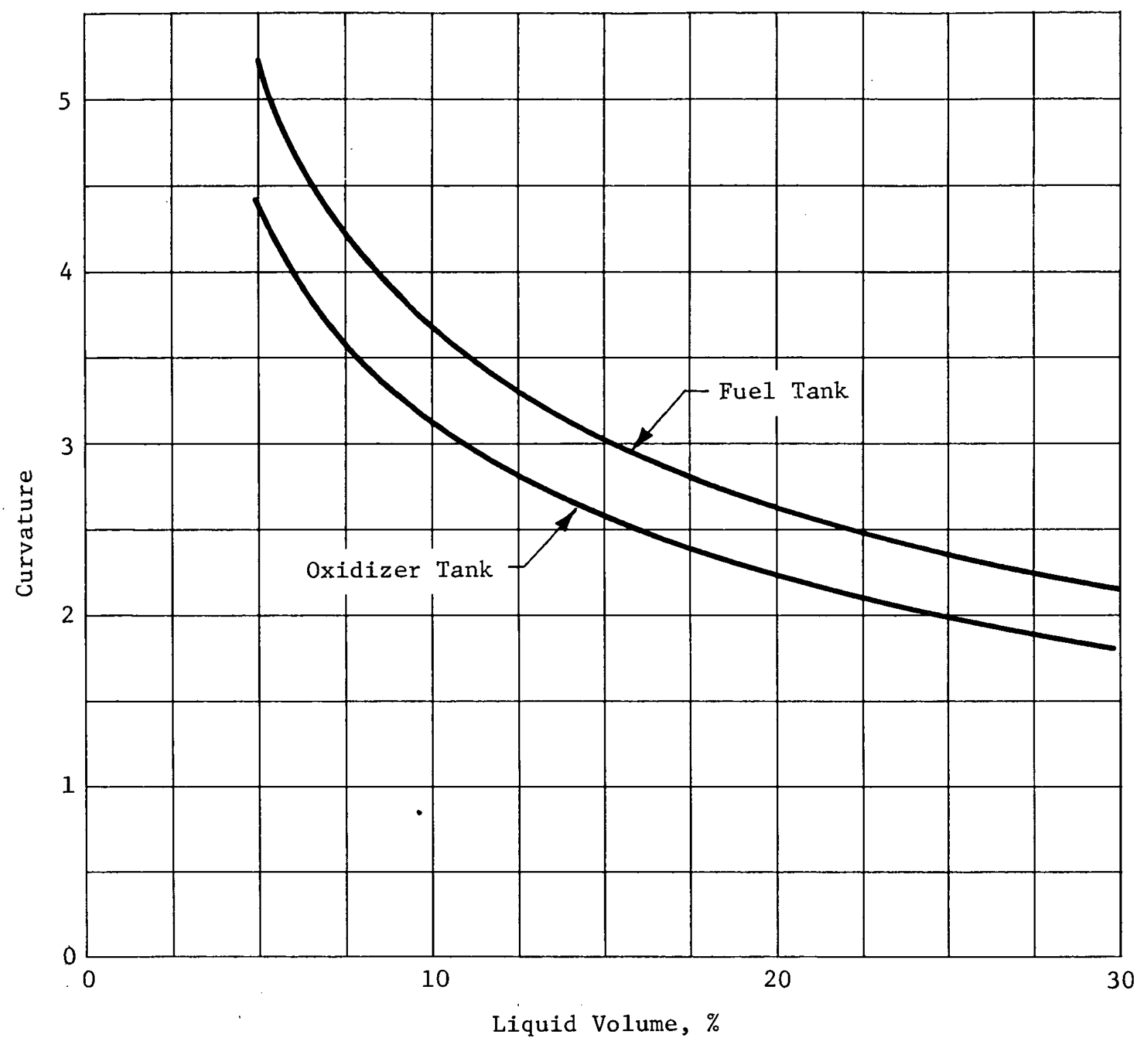

Fig. VI-12 Curvature vs Volume of Liquid within Vanes 
b. Fuel Tank - The design and operation of the standpipe for the Euel tank is essentially the same as that for the oxidizer tank, except ground hold cooling is not required for the fuel tank. The possible standpipe volumes for a device height of $1.4 \mathrm{R}$ are shown in Fig. VI-13. Modular installation of the device restricts the diameter of the top of the standpipe to about $4 \mathrm{in}$. This means that the volume of the standpipe must be less than $1.5 \%$ of the tank volume. A standpipe with a $3^{\circ}$ taper and a volume of $0.84 \%$ was selected from both operation and tank installation considerations.

Curvature of the interface inside and outside the standpipe versus volume was included in Fig. VI-1I and VI-12. Curvature inside the standpipe is large enough to keep the standpipe full except for very low liquid volumes (approximately $3 \%$ or less).

The operation of the devices in the fuel and oxidizer tanks is summarized with Fig. VI-14, which shows liquid orientation at various points during the mission. Three dimensional interfaces are represented in two dimensions by showing a representative cross-section of the 1iquid. In Figure VI-14(a), the positioning of the initial ullage volume is shown. Near the beginning of the mission, the booms are deployed, displacing the ullage as shown in Fig. VI(b). Following deployment, the ullage will return to the position shown in Fig. VI-14(a). In (c), (d), and (e) of Fig. VI-14, the position of the liquid is shown as it is depleted. Before the last burn, a small amount of liquid remains as shown in Fig. VI-14(f).

3. Communication Channel

If some liquid were to become located at the top of the tank, out of contact with the device, there are no forces acting to return the liquid to the acquisition device. The device was designed to always keep the liquid oriented within the vanes, but some unexpected disturbance could allow come of the liquid to be displaced from the device. Vaporization and condensation (heat pipe effect) could transfer 1iquid from about the device to the top of the tank. 


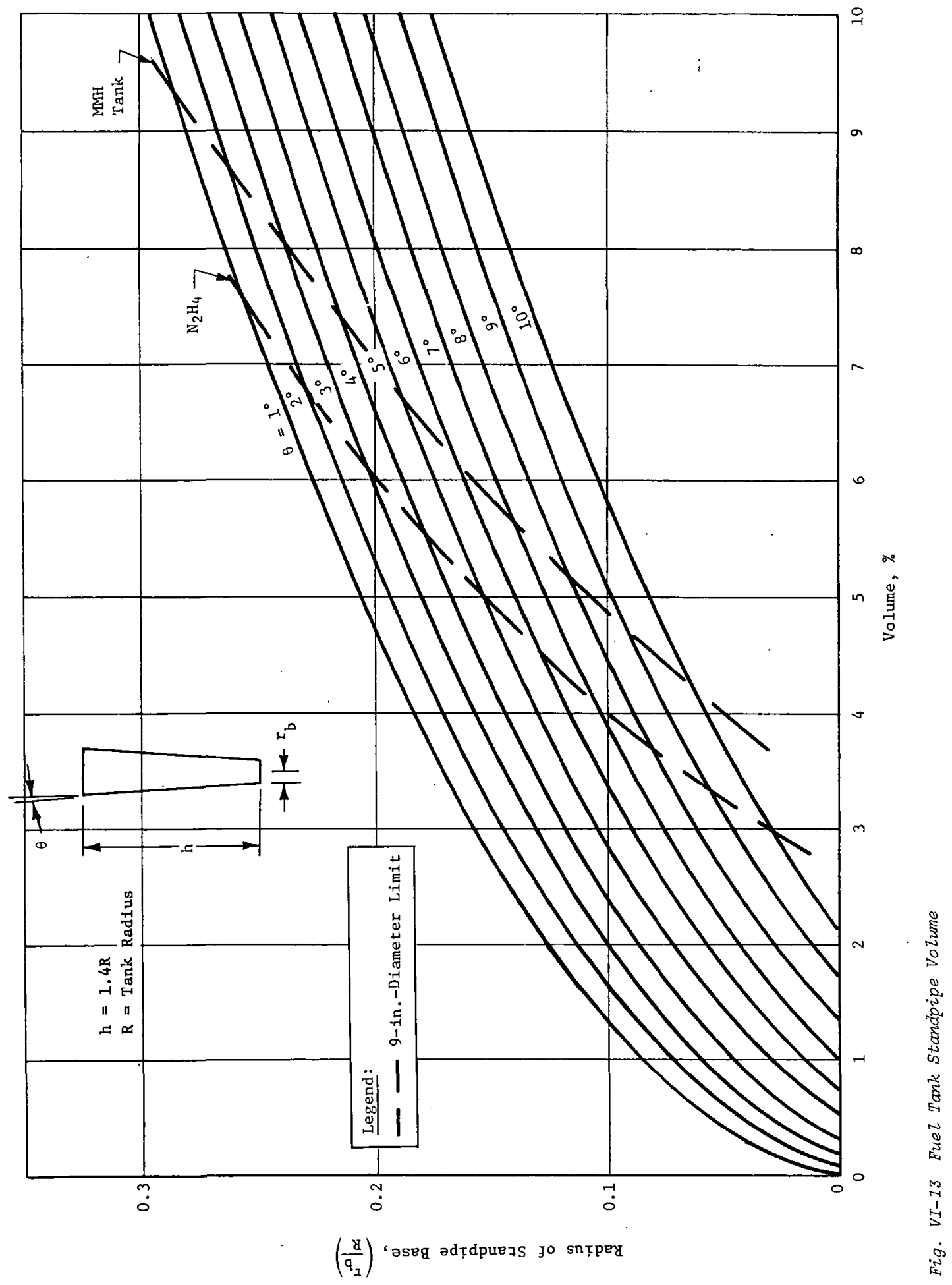

$V I-42$ 

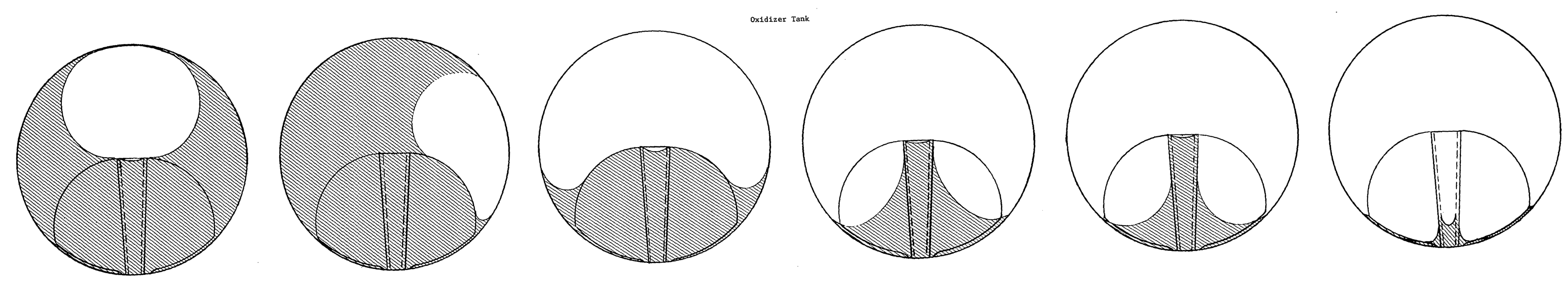

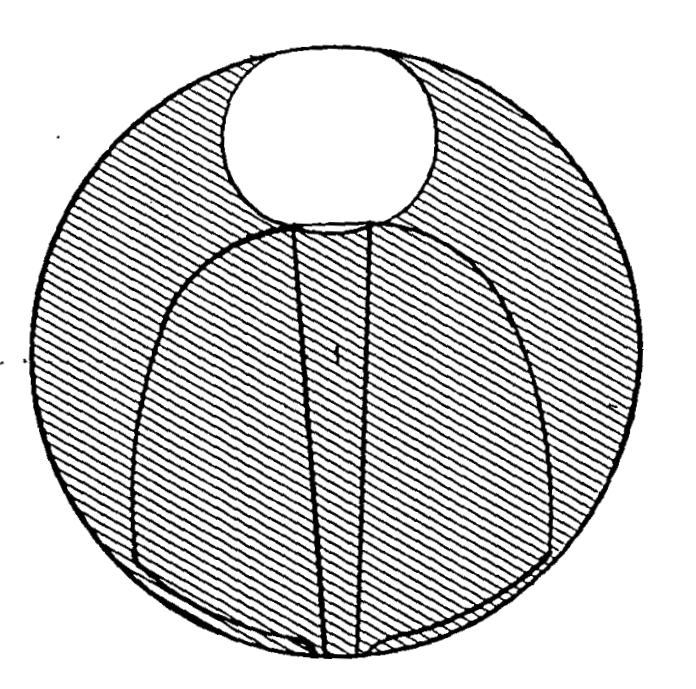

(a) Intitial v1lage, zero 8

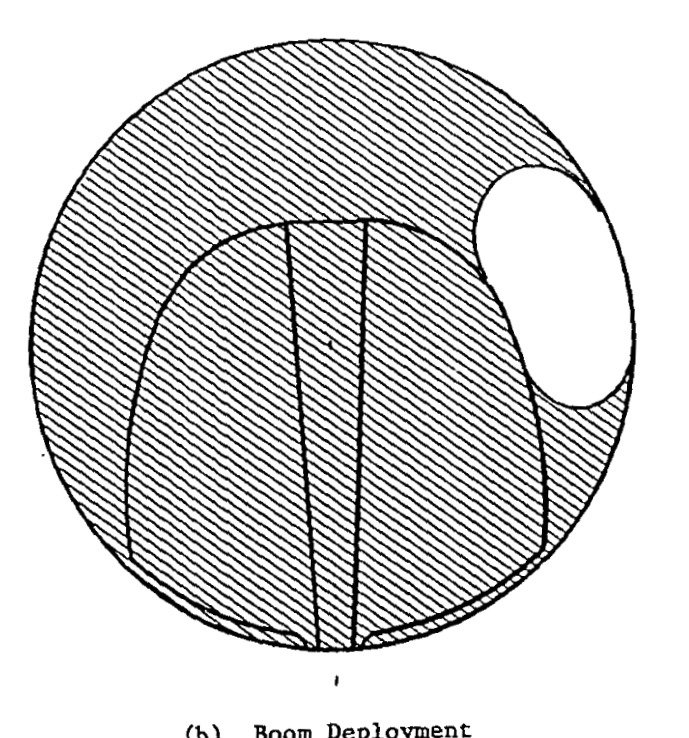

(b) Boom Deployment

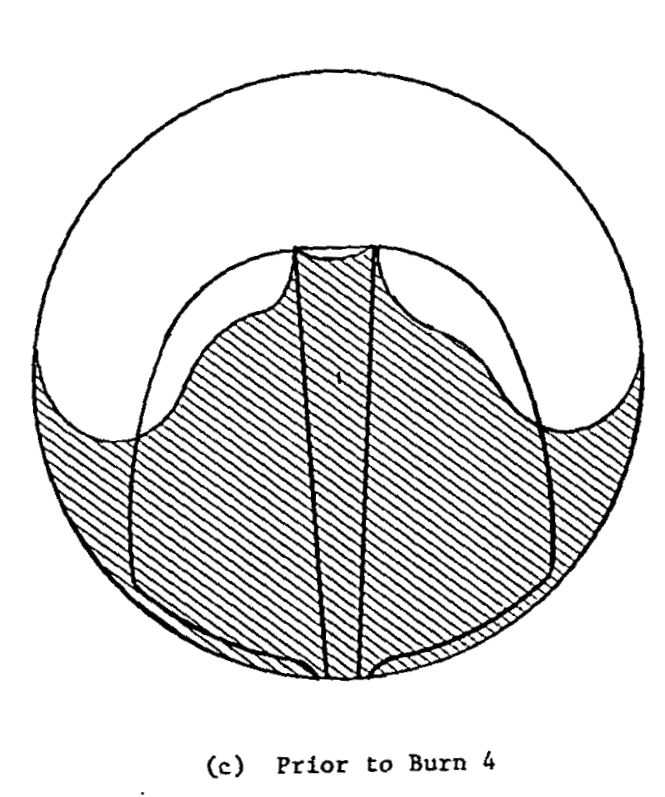

(c) Prior to Burn 4
Fuel Tank

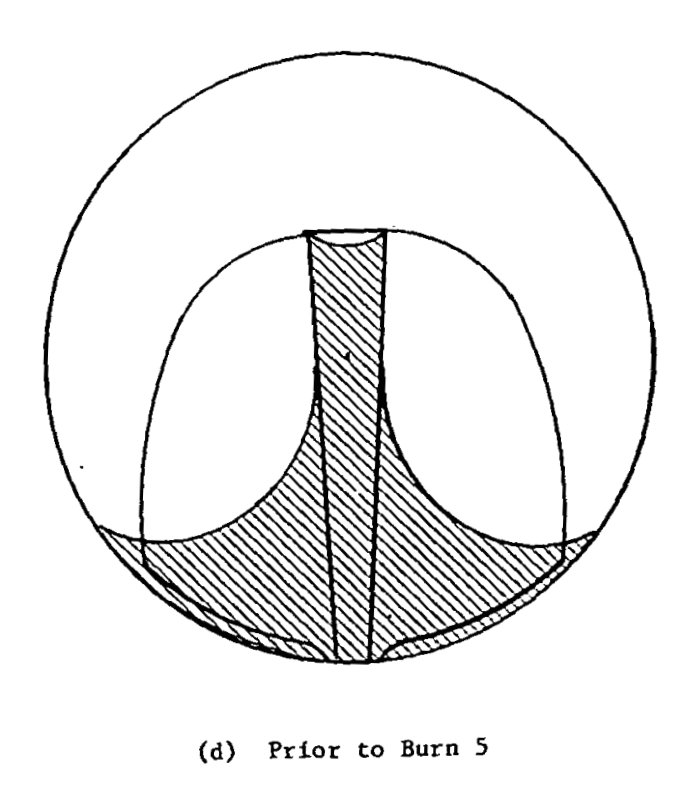

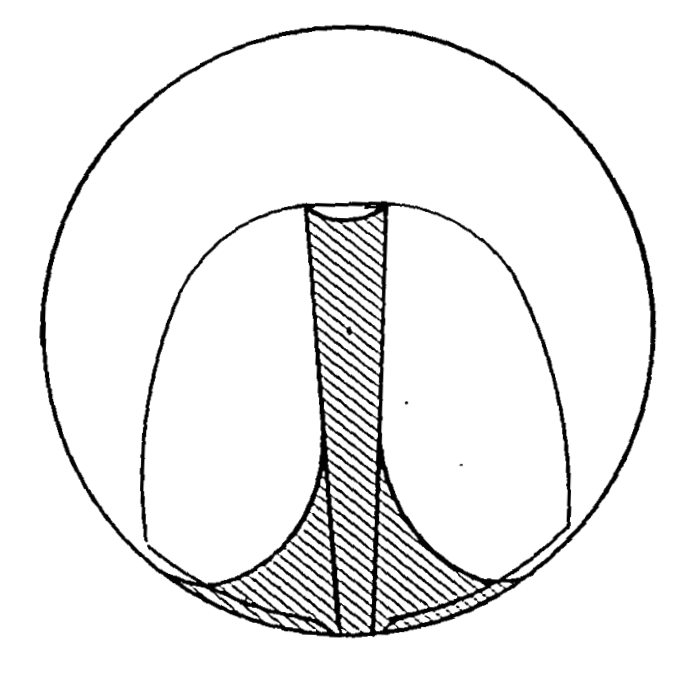

(e) Following orbit Insertion$$
\text { Fig. }
$$

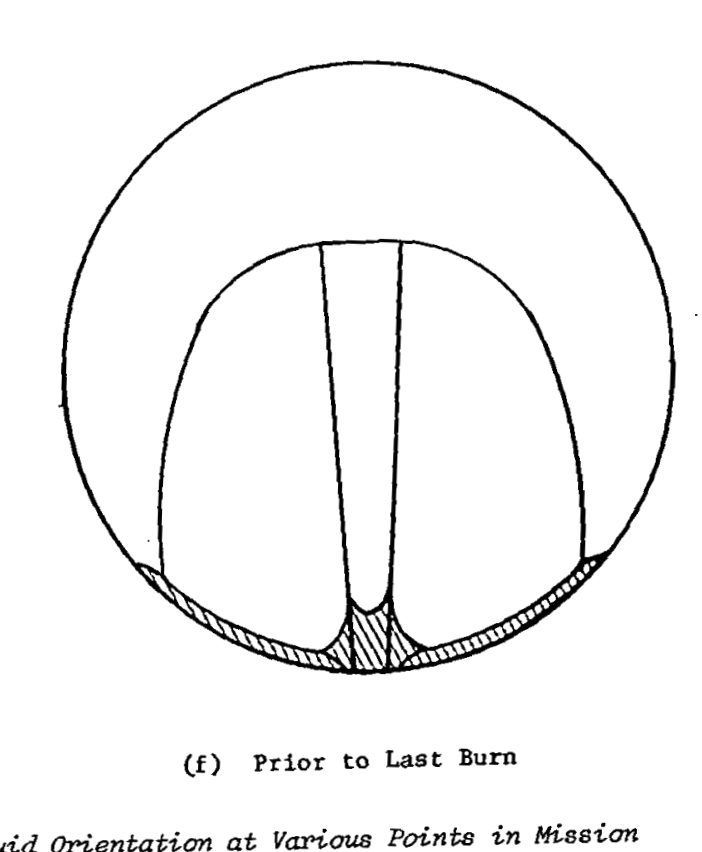

FOLDOUT FRAME 
Under low-g conditions, both volumes of liquid (that about the device and the displaced liquid) must be rather small or they will contact each other, especially if the contact angle is near zero. If the two volumes are in contact, liquid will return to the device due to the difference in capillary forces. A communication channel was added to the device to provide for the return of the liquid when the two interfaces are not in contact.

The operation of the communication channel is discussed in Chapter III. It was shown that a vaned device will produce a relatively large capillary pressure difference between displaced liquid and liquid about the device, even if the contact angle was as large as $5^{\circ}$. Return of displaced liquid in a reasonable amount of time was shown to be possible.

Consider now the selected channel for the standpipe with vanes. It is a U-channe1, 1-in. wide and 1/2-in. high. A more general form of the equation for the flow in the channel, based on the Darcy equation for flow in a channel and assuming laminar flow, will be used here.

$\mathrm{Q}=\frac{\pi \Delta \mathrm{P} \mathrm{D}^{4}}{128 \mu \mathrm{L}}$

where

$\Delta \mathrm{P}=$ pressure difference across the length of the channe1,

$\mathrm{D}=$ hydraulic diameter ( 4 times the area divided by wetted perimeter),

$\mu=$ viscosity,

$L=$ length of the channel.

Based on the Chapter III analysis, a conservative estimate for the $\Delta \mathrm{P}$ is obtained by assuming a difference in curvature, between the two interfaces, of 2 . The worst-case capillary pumping conditions were shown to exist in the Flox tank. Under these conditions, liquid would be pumped at a rate of $0.19 \mathrm{lb} /$ minute. Hydrostatic pressure, due to coast accelerations of $10^{-7} \mathrm{~g}$ could be acting against the capillary pressure pumping the liquid. This pressure was found to be at least an order of magnitude less than the capillary pressure, so its effect will be neglected. 
The rate at which propellant would be transferred by vaporizationcondensation can be estimated from the heat input to the tank. The following heat inputs were discussed in Chapter III:

Tank supports, $2.0 \mathrm{Btu} / \mathrm{hr}$;

Feed line, 0.5 Btu/hr;

Solar (with loss of $1 \mathrm{ft}^{2}$ insulation), $387.0 \mathrm{Btu} / \mathrm{hr}$.

The input due to heat soakback was determined to be $62.0 \mathrm{Btu} / \mathrm{hr}$ using Ref. VI-4. While this is only an intermittent heat input, it will be considered as continuous for this analysis. Summing the heat inputs gives a total of $452 \mathrm{Btu} / \mathrm{hr}$ entering the tank. If all of this heat was used to vaporize liquid located over the tank outlet, the rate of vaporization would be $0.0921 \mathrm{~b} / \mathrm{min}$.

It is assumed that all the vapor produced condenses at the cooler, top of the tank. The rate at which liquid would be transferred in the channels due to capillary pumping $(0.191 \mathrm{~b} / \mathrm{min})$ is greater than the vaporization rate by $0.0981 \mathrm{~b}_{\mathrm{m}} / \mathrm{min}$. Therefore, a single communication channel will be capable of returning liquid under the worst-case heat input conditions and with a conservative estimate of the capillary pumping pressure. This capability eliminates the loss of liquid due to vaporization-condensation as a concern.

One of the most likely causes of a disturbance, which could overcome the capillary retention capability of the device and displace liquid, is the operation of the spacecraft engine. For example, during a short-duration burn, the momentum imparted to the liquid due to settling may still be present at the end of the burn. The continued motion of the liquid after engine shutdown could cause liquid to move away from the device. If such a disturbance were to remove all the liquid from the acquisiton device, the channel must return enough liquid to start the engne and settle propellant at the next burn. A volume of liquid equal to $1 \%$ of the tank volume should be adequate to start and settle. Using the above calculated flowrate for the channel, it would take $2.3 \mathrm{hr}$ to return $1 \%$ of the propellant. A minimum time between burns is not specified for the mission, but a period of about $3 \mathrm{hr}$ should not restrict the operation of the spacecraft. 
The effect of the standpipe has been ignored in the above discussion.' It is very unlikely that any disturbance would cause a loss of liquid from the standpipe, so its volume of liquid will always be available at the outlet. Even if the standpipe had to refill, it will produce a large capillary,pressure difference and would refill rapidly.

A single communication channe1, running from the top of the tank to the device, is adequate for the standpipe with vanes device. As discussed above, one channel will return liquid to the device at a rate sufficient to maintain enough propellant at the tank outlet. It is possible to have small puddles of liquid that do not contact the channel or the liquid about the device. The reservoir of liquid in the standpipe will compensate for any such weaknesses of the channel.

One of the alternative oxidizer tank designs has the ground hold cooling tubes located on the interior wall of the tank. These coolant tubes form four communication channels. While a tube is not as effective a channel as a $U$ section, four of the tubes would provide sufficient liquid transfer capability.

4. Outlet

The outlet region of the tank provides the transition from the acquisition device to the feed line. It combines the flow from about the vanes with that from the standpipe and directs it to the feedline during engine operation. Under low-g conditions, liquid will tend to orient toward the outlet. Communication within the outlet to the standpipe enables the standpipe to refill.

The need. for modular installation has a large influence on the design of the tank outlet. Mounting flanges for the device and clearance for the tank installation must be included. An offset feed line becomes necessary. The all-welded alternative design for the oxidizer tank illustrates how much simpler the outlet could be if modular installation were not required.

At low liquid volumes, the outlet configuration can affect the operation of the acquisition device. Communication must exist between the communication channel, vanes, standpipe, and feed line. This was achieved by extending the vanes into the base of the outlet, adding fins on the base of the standpipe, and by aligning the communication channel with a vane. Some gaps are necessary. for fabrication reasons, such as the gap between the communication 
channel and the vane. Liquid will easily bridge the gaps if the spacing is kept small. In the all-welded alternative design, components of the device are welded directly together and joined to the tank wall to eliminate the gaps.

A liquid interface within the outlet region has a stability approximately equal to that of an interface inside the standpipe. Both the vanes of the device and the fins at the base of the standpipe provide the stability. Throughout the mission, the outlet should remain full of liquid.

When the volume of liquid is less than $2 \%$, the outlet is full of liquid and the standpipe is partially full. At this point in the mission, a number of short-duration burns are necessary. The occurrence of suction dip and its effect on the expulsion efficiency of the device become a concern. The last burn imposes the worstcase conditions with respect to the liquid expulsion capability of the device.

The last burn volumes for each propellant are listed in Table VI-1.

Table VI-1 Last Burn Volumes

\begin{tabular}{|l|l|l|l|l|}
\hline & Flox & $\mathrm{MMH}$ & $\mathrm{F}_{2}$ & $\mathrm{~N}_{2} \mathrm{H}_{4}$ \\
\hline Mass, $1 \mathrm{~b}$ & 3.8 & 1.5 & 16.6 & 8.0 \\
Volume, $\mathrm{ft}^{3}$ & 0.042 & 0.028 & 0.18 & 0.13 \\
Volume, \% & 0.31 & 0.38 & 1.70 & 1.22 \\
\hline
\end{tabular}

At these volumes, a careful accounting of the location of the liquid is required. The communication channel will never be completely empty of liquid under low-g conditions. Up to $0.2 \%$ liquid volume could be located in the channel. Some liquid will also be held in the joint between the vanes and the standpipe. Before the last burn, a considerable fraction of the remaining liquid would not be oriented directly at the outlet. Liquid at the outlet would be used to start the engine and all the liquid oriented away from the outlet will begin to settle. If there was only enough liquid remaining to accomplish the last burn, ingestion of gas would occur at some point prior to depletion of the liquid. One hundred percent expulsion efficiency is not possible, but at least $99+\%$ is feasible. Therefore, the ability of the acquisition device to accomplish the last burn depends on how much of the loaded propellant has been used before that burn. A $4 \%$ margin is added to the propellant load to compensate for variations in loading accuracy, propellant outflow rates, engine performance, etc. 
If some of this extra propellant is available at the last burn, so there would be $1 \%$ instead of just $0.3 \%$, the probability of accomplishing the last burn would be greatly increased.

An offset outlet aggravates the suction dip problem and would give a lower expulsion efficiency than an outlet located on the tank centerline. The velocity profile is biased toward the side of the tank on which the outlet is located, but the liquid interface is symmetrical. Suction dip will leave some liquid stranded on the opposite side of the device from the outlet. In the allwelded design, the outlet is on the tank centerline and the contour of the outlet reduces the flow velocities and produces a uniform velocity profile.

The actual expulsion efficiency of the device can be best determined experimentally. First, the location of the liquid before the last burn would be established by testing subscale models of the outlet in the drop tower. Then one-g draining tests would be accomplished by using representative initial conditions and scaling the liquid flowrate and model dimensions. Once the expulsion efficiency was determined, the amount of liquid required to accomplish the last burn would be known. If the $4 \%$ margin was not adequate, it could be increased to 4.5 or $5 \%$ to ensure a successful last burn.

\section{Pressurization/Vent Tube}

The tank is pressurized and vented through a single port located at the top of the tank. Pressurization is accomplished before and during each engine burn. A typical pressurization system operates with a regulator that senses the downstream pressure in the pressurization inlet. If the ullage volume is in contact with the pressurization inlet, pressurant gas flows into the tank until the desired tank pressure is reached. If liquid was over the inlet or inside the line, the regulator would be responding to pressurant within the line. As the liquid is pushed out of the line, the pressurant would be compressed, giving a false indication that the proper tank pressure had been reached. Erratic pressurization would occur. There is also the possibility that the vent system, which uses the same line as its inlet, may sense the pressure surges as a need to vent the tank.

The vent system is for emergency relief of the tank pressure only. It is not expected that the tanks would have to be vented during the mission. If venting should be required, the ullage volume should be in contact with the inlet so that only gas is vented. 
The acquisition device was designed so that it will keep the ullage volume located at the pressurization/vent port. The port is a tube that extends about one-third of the distance between the top of the tank and the top of the device. Its diameter is small enough to prevent it from interfering with the centering of the ullage bubble. Some liquid will collect about the port where it joins the tank wall, but the length of the tube ensures that its tip will be free of liquid.

As the pressurant enters the tank, forces due to the flow of the gas will exceed the capillary forces at the interface. A diffuser is incorporated into the lower end of the port. Angled holes in the wall of the tube will diffuse the gas flow in a radial direction. An example of an approach to the design of the diffuser for the port, as it was accomplished for the Viking orbiter Propulsion system, can be found in SE004-47-02.* Some disturbance of the interface is expected, but a gross displacement of the liquid from the device will be avoided with this type of diffuser. However, additional analysis is required in this area. Bubbles may be created during pressurization. The analysis of bubble ingestion showed that unless the bubbles are very close to the outlet before engine start they will not be ingested.

When the tank is vented, there will be some disturbance of the interface due to the reduction in the gas pressure. Variations in pressure within the ullage region will cause the shape of the interface to be chanted. Development of an analytical model is needed in this area. Tests would be used to determine the actual effect of pressurization and venting on the liquid interface, unless analytical tools capable of determining such effects are developed.

6. Design Summary

The design approach placed the most emphasis on obtaining gas-free liquid expulsion. This was accomplished by using only six vanes, the selected vane profile, and a standpipe. Over the entire range of liquid volumes these factors help to keep the liquid well oriented at the tank outlet. Such problems as suction dip, slosh, and bubble ingestion are all minimized by this approach. The number of vanes and the profile is still adequate to center the bubble for venting, pressurization, and control of the center of gravity.

*"Viking Orbiter 75 Propulsion System, Propellant Pressurization Diffuser Design Analysis." SE004-47-02. Martin Marietta Corporation, Denver, Colorado, June 20, 1972. 
C. OTHER SYSTEM CONSIDERATIONS

1. Dual-Mode Propulsion System

The same design for the standpipe with vanes is applicable to either of the two oxidizers being evaluated. While the size of the Flox and fluorine tanks is different, both devices would be proportional in size.

The Flox/MMH combination has a bipropellant engine, but the $\mathrm{F}_{2} / \mathrm{N}_{2} \mathrm{H}_{4}$ combination uses a dual-mode propulsion system. It can operate in either the monopropellant or bipropellant mode. When operating in the bipropellant mode, the engine still starts and operates in the $\mathrm{N}_{2} \mathrm{H}_{4}$ monopropellant mode for $5 \mathrm{sec}$ before switching to bipropellant operation. During the 5-sec period, some settling of the $F_{2}$ would occur. This would reduce the problem of orienting liquid at the outlet, but it is not considered adequate to operate the $F_{2}$ tank without an acquisition device. Settling of small liquid volumes could take more than $7 \mathrm{sec}$ if there was no device in the tank. Also, the need for cg control and provisions for pressurization and emergency venting require an acquisition device in the $\mathrm{F}_{2}$ tank.

The $\mathrm{N}_{2} \mathrm{H}_{4}$ tank acquisition device must provide propellant for both the attitude control system (ACS) and the trajectory correction engine. Propellant for the ACS would be tapped off the feed line downstream of the tank outlet. Liquid will always be located at the outlet, so the relatively small demands of the ACS would be provided. It has been shown that the disturbances due to the ACS maneuvers will not cause any significant disturbance of the liquid. A supply of propellant would be maintained during the maneuver.

2. $\mathrm{OF}_{2} / \mathrm{B}_{2} \mathrm{H}_{6}$ Propellant Combination

Acquisition devices were not specifically designed for the $\mathrm{OF}_{2} / \mathrm{B}_{2} \mathrm{H}_{6}$ propellant combination. In Chapter $V$, the properties of the three propellant combinations were compared. The comparison shows that the properties of these propellants are similar to those of the other combinations. In terms of stability and pumping capability, the differences are small. Assuming that the spacecraft criteria of the $\mathrm{OF}_{2} / \mathrm{B}_{2} \mathrm{H}_{2}$ propulsion system would be essentially the same as those of the other combinations, the same devices could also 
be used for this propellant combination. The material compatibility considerations for the $\mathrm{OF}_{2} / \mathrm{B}_{2} \mathrm{H}_{6}$ propellant combination are similar to those for the other propellants. Aluminum or stainless steel would be used with $\mathrm{OF}_{2}$ and, because of weight, titanium would be used with $\mathrm{B}_{2} \mathrm{H}_{6}$.

3. In-Line versus Side-by-Side Tanks

Throughout the design and analysis of the acquisition device, the impact of in-line tanks instead of side-by-side tanks was considered. The effect of the two configurations on the location and travel of the center of gravity is discussed in chapter III. The maximum shift in the $\mathrm{cg}$ was found to be essentially the same for both configurations. However, the difference in the location of the spacecraft $\mathrm{cg}$ with respect to the ACS thrusters resulted in tank accelerations for the in-line configuration that were about twice those for the side-by-side configuration. These acceleration values were presented previously in the spacecraft criteria section of Chapter I (Table I-2). The difference between the two cases is not considered significant; however, the larger accelerations associated with the in-line tank configuration were used in designing the device.

Other than the above mentioned effects, the operation of the acquisition device and its interaction with the various spacecraft subsystems remains the same for either tank configuration.

4. Cylindrical versus Spherical Tanks

The selected acquisition device concept is adaptable to a cylindrical tank. The device height and the vane profile would be designed to account for the cylindrical tank geometry. It is assumed that the mission and spacecraft criteria would not be changed significantly if the spherical tanks were replaced with cylindrical tanks.

With a cylindrical tank, the distance between the pressurization/ vent port and the feed line is greater than it is for a spherical tank of the same volume. This is an advantage with respect to keeping the ullage away from the tank outlet, but it is a disadvantage with respect to providing gas-free liquid to the engine. A taller device means that more liquid will be located up within the vanes, removing liquid from the vicinity of the outlet. Settling, slosh, and suction dip are all more likely when this happens.

VI -52 
A cylindrical tank has some inherent stability, while a spherical tank does not. Liquid inside a bare spherical tank will reorient under the effect of any acceleration, regardless of its magnitude. Liquid in a cylinder, or the barrel section of a cylindrical tank, however, will remain oriented when an axial acceleration is tending to displace it. The critical Bond number, based on the radius of the cylinder, is 0.84 when the contact angle is zero. For example, Flox will remain oriented in a 2-ft-diameter cylinder when the axial acceleration is less than $1 \times 10^{-5} \mathrm{~g}$. The stability of the tank will supplement the stability of the acquisition device. Other than the above mentioned differences, the operation and performance of the device would be essentially the same.

5. Ground-Hold Cooling System and Temperature Sensor Integration

A ground hold cooling system was integrated into the design of the standpipe-with-vanes device. Channels located in the wall allow the $\mathrm{LN}_{2}$ coolant to be circulated through the standpipe. The vanes act as heat exchanger fins.

The standpipe is divided into three sectors, manifolded at the top. Each sector has a tube passing through the bottom of the tank; coolant enters one tube and leaves through the other two tubes. No thermal analysis of the cooling system was performed, so the flow area required and the efficiency of the device as a heat exchanger need to be determined.

In one of the alternative designs, the coolant tubes are used as communication channels. The tube is a single loop, entering and leaving the tank top, which forms four communication channels. Again, the design is only conceptual. A complete thermal analyn sis is required to size the cooling system.

An externally mounted ground-hold cooling system is suggested as another alternative. Cooling coils could be directly attached to the outside tank wall. Thermal performance of such a system tends to be better than an internal system because heat entering the tank is intercepted at the wall. The most important advantage of the external system is improved safety and reliability. An external system does not have to penetrate the tank and is not in direct contact with the propellant. Studies of cooling systems for use with spacecraft using fluorine recommended external systems for those reasons ( $\operatorname{Re}$ VI-9, VI-10, and VI-11). 
Another advantage of external cooling is that the tank could be loaded without venting. Tank pressure can be controlled by precooling the tank and maintaining coolant flow during loading (Ref VI-12).

Temperature sensors are located in both the fuel and oxidizer tanks. A sensor integrated into the standpipe would minimize the effect of the sensor on the operation of the device. Since the typical sensor would be small, its location in the tank would have little effect on the orientation of the liquid.

6. Flexibility

The selected standpipe-with-vanes system is a flexible concept that could be applied to most typical deep-space missions. As discussed, the tank configuration and geometry can be varied without affecting the operation of the device significantly. Another important factor influencing the flexibility of the concept is the initial ullage volume. Devices were designed for $5 \%$ and $20 \%$ ullage volumes, but $50 \%$ ullages are typical in propulsion systems using a blowdown pressurization system.

In the analysis of the design, it was shown how the height of the device and the profile of the vane are primarily determined by the initial ullage volume. Based on the height and profile, performance capabilities such as stability and ability to expel gas-free liquid are established. Design of a device for a larger ullage volume, such as $50 \%$, would proceed in a similar manner. Analysis of this device would show that most aspects of its operation were less critical due to the larger initial ullage volume. Since the device is smaller in height, liquid would tend to orient closer to the outlet. Larger numbers of vanes could be used to improve the stability of the system without degrading the orientation of liquid at the outlet. A simple, triangular vane profile would be adequate for centering the ullage bubble. Due to the size of the bubble, lateral accelerations would cause some displacement, but liquid could not cover the pressurization/vent port. A smaller device, triangular profile, and larger number of vanes would improve the ability of the device to expel gas-free liquid. A standpipe would be too small and not really essential in such an application, so it would not be incorporated. An acquisition device of this type was designed in the previous contract (NAS7-754). Engineering drawings showing its configuration can be found in Fig. V-19 of Reference VI-4. 
7. Effects of Space-Storable Propellants

The space-storable propellants considered, Flox, $\mathrm{F}_{2}, \mathrm{OF}_{2}$ and $\mathrm{B}_{2} \mathrm{H}_{6}$, have a strong influence on the design and fabrication of a spacecraft propulsion system in comparison to a system employing earth storables. In previous discussions, some of the factors have been discussed, but there are many others. The following is a compilation of factors that should be considered when a system is designed for use with space storables.

Fabrication and Assembly - Selected materials must be compatible. The use of dissimilar materials within the tank is discouraged because this can increase the corrosion rate. All-welded fabrication is preferred; joints should not produce contaminant trap areas. Faying surfaces should be eliminated, and adequate clearance must be provided for cleaning and passivation. Stringent cleaning procedures must be used.

Ground Hold and Launch Operations - A clean and dry system is required for fluorine-based propellants. The propellant system must be passivated with gaseous fluorine before loading. Loading, transfer, and mating to the launch vehicle are all hazardous operations with fluorine propellants and stringent control is required. Any leakage is unacceptable. The vapor is too toxic to vent directly to the atmosphere; a charcoal burner should be employed. Cooling must be provided during ground hold due to the low storage temperature.

Mission Operation - The thermal implications presented by spacestorable propeliants can be greater than with an earth-storable system. Heat rejection may be needed in space, depending on the mission, heat leaks, method of pressurization, etc. The probability of venting is greater and is also more difficult. Reliable relief valves are not now available. Some work on rupture discs was recently completed (Ref VI-13). As discussed previously, pressurization presents some difficulties. Flightweight valves and regulators are problems. A worst-case situation is presented by the fluorine-containing oxidizers because of taxicity, reactivity, temperature, and propellant-property considerations.

\section{Contact Angle}

The contact angle between any of the propellants considered and any of the metals used to fabricate the tank and device is essentially $0^{\circ}$, provided the propellant is not contaminated and the 
metal is clean. If the propellant should decompose or become contaminated, or if the metal has any films or residues on its surface, the contact angle can increase.

An increase in contact angle will degrade some of the aspects of the operation of the acquisition device. This increase would have two interdependent effects on the shape of an interface in low-g:

1) When the contact angle is increased, the curvature of the interface is decreased;

2) The tank surface area covered by a given volume of liquid is reduced as the contact angle increases.

The effect of these two factors on the operation of the communication channel is discussed in Chapter III. Liquid displaced from the device is less likely to be in contact with liquid about the device at a larger contact angle due to the second effect. A reduction in curvature means that the capillary pressure pumping the liquid in the channel is decreased. It was shown that the pumping pressure can be reduced to zero, or actually reversed as the contact angle increases. The post was found to become ineffective at a $2^{\circ}$ contact angle; a standpipe would still be effective at a contact angle of $10^{\circ}$; but, the standpipe with vanes would be capable of returning displaced liquid at contact angles of $20^{\circ}$ or more.

Some of the effects of a larger contact angle are advantageous. Due to the second effect, liquid would be more closely oriented over the outlet as the contact angle increases. Stability of the interface increases as the contact angle increases. For example, consider the interface in a cylinder. At a $0^{\circ}$ contact angle, the critical Bond number is 0.84 . The critical Bond number increases as the contact angle increases, reaching a maximum of 3.39 at a contact angle of $90^{\circ}$.

Since an increased contact angle can degrade some aspects of the performance of the device, the approach is to take all precautions to keep the contact angle as close to zero as possible. Conditions affecting the cleanliness of the tank and the propellant can be controlled during fabrication, assembly, and launch operations to guarantee a negligible contact angle. During a longduration mission, factors may tend to increase the contact angle. The amount of increase that could be expected is unknown; further evaluation is required. 
VII. CONCLUSIONS AND RECOMMENDATIONS

A. CONCLUSIONS

Based on the analytical and experimental evaluations of a variety of candidate surface tension propellant acquisition concepts conducted under this program, the Fruhof-class of propellant acquisition systems offers the best approach to a universal system for interplanetary spacecraft. These systems provide a wide operating band and can be tailored to specific applications with the desired operational margin. The Fruhof standpipe-with-vanes system is preferred for further design study, because this system appears most capable of meeting the spacecraft and mission requirements of the type evaluated.

A preliminary design of the device was accomplished, based on ana1ysis and testing of the more significant factors influencing its fabrication and operation. However, further effort would be required to tailor the device to a specific propulsion system and mission. This effort is necessitated by both fluid mechanics considerations, which could not be treated in sufficient detail in the scope of this contract, and the use of space-storable propellants. These propellants, in general, and fluorine-containing oxidizers, in particular, present a worst-case situation for propellant acquisition, which is at least an order of magnitude more difficult than an earth-storable propellant situation.

More information and improved analytical tools are required in the areas of propellant reorientation/ullage positioning, propellant settling and slosh dynamics, and the impact of pressurant inflow and vapor venting on the liquid/vapor interface configuration. Additional information is also required on the effect of both time and contaminants on the contact angle between the propellants and the tank and acquisition device materials. An evaluation of device fabrication and tank installation techniques is also needed. 
B. RECOMMENDATIONS

The following recommendations outline a plan for further development of the propellant acquisition system:

1) Some means of calculating the shape of a zero-g interface about a vaned standpipe acquisition device should be developed. The tradeoffs between developing techniques to calculate the actual three-dimensional interfaces or developing approximate methods that would give a suitable two-dimensional representation of the actual interface should be evaluated.

2) Once the interface can be determined, the analysis should be extended to consider the effect of an acceleration acting on that interface. Both the axial and lateral stability of the device could be determined. As a part of this analysis the pumping capability of the vanes and the location of the liquid under the effect of an acceleration would be established.

3) The effect of the diffuser design on the shape of the interface during pressurization should be analyzed.

4) The pressure gradients produced within the ullage due to venting and their effect on the interface shape should be analyzed.

5) A complete flow analysis of the outlet region of the tank, aimed primarily at the smaller liquid volumes, should be accomplished. Effects, such as settling, suction dip, bubble ingestion, and the combined flow from the standpipe and the bulk region should be included.

6) Drop tower tests of the selected concept should be accomplished to verify and supplement the analytical effort. Scale models of the device would be tested to determine interface shapes and their stability. A model of just the outlet region of the tank would help to verify the operation of the device at small liquid volumes.

7) One-g draining tests, using representative initial conditions, would assist in verifying the operation of the device while the spacecraft engine is operating. These should be conducted. The expulsion efficiency and the pressure losses due to flow through the outlet could be established. 
8) A KC-135 aircraft flying a low-g trajectory would provide sufficient test time to qualitatively verify certain propulsion system events. A subscale tank model should be used to demonstrate pressurization, venting, and outflow.

9) After the above analytical and experimental effort, the design of the device should be revised to incorporate any improvements. Based on this design, a prototype of the device should be built to evaluate fabrication and assembly techniques and to develop inspection procedures. The device should be used to demonstrate tank installation and removal procedures. Testing to demonstrate structural integrity and one-g outflow with the actual propellants should then be conducted.

At the completion of this planned program, the device would be proven with respect to its operation and fabrication. Manufacture of the actual hardware could then begin. 
VIII. REFERENCES

Chapter I

I-1 H. L. Paynter, et al.: Investigation of Space Storable PropelZont Acquisition Devices, Final Report, Volume I. MCR-70-171 (Vo1 I). Martin Marietta Corporation, Denver, Colorado, October 1970 .

I-2 H. L. Paynter, et al.: Investigation of Space Storable PropelLant Acquisition Devices, Final Report, Volume II. MCR-70-171 (Vol II). Martin Marietta Corporation, Denver, Colorado, December 1970 .

I-3 W. A. Hasbach: Lightweight Solar Panel Development. Technical Report 32-1519, Jet Propulsion Laboratory, California Institute of Technology, Pasadena, California, 15 March 1971.

I-4 D. A. Fester: Space Storable Propelzant Acquisition System Project Work PZan. MCR-71-231. Martin Marietta Corporation, Denver, Colorado, August 1971.

Chapter II

II-1 H. L. Paynter, et al.: Investigation of Space Storable Propellant Acquisition Devices, Final Report, Volume II. MCR-70-171 (Vo1 II). Martin Marietta Corporation, Denver, Colorado, December 1970 .

II-2 D. A. Fester and P. E. Bingham: Evaluation of Fine Mesh Screen Device in Liquid Fluorine. R-70-48631-010. Martin Marietta Corporation, Denver, Colorado, June 1970.

Chapter III

III-1 H. L. Paynter, et al.: Investigation of Space Storable Propelzant Acquisition Devices, Final Report, Volume II. MCR-70-171 (Vol II). Martin Marietta Corporation, Denver, Colorado, December 1970.

III-2 H. L. Paynter, et al: Experimental Investigation of Capizlary Propelzant Control Devices for Low-Gravity Environments. MCR-69585. Martin Marietta Corporation, Denver, Colorado, June 1970. 
III-3 J. Happel and H. Brenner: Low Reynold's Number Hydrodynomics. Prentice-Hall Incorporated, Englewood Cliffs, New Jersey, 1965.

III-4 Thermoelectric Outer Plonet Spacecraft Advanced System Technology Functional Description. Jet Propulsion Laboratory, Pasadena, California, 17 September 1971.

III-5 P. Concus, et al.: Small Amplitude Lateral Sloshing in Spheroidal Containers Under Low Gravitational Conditions. NASA CR-72500. Lockheed Missile and Space Company, Sunnyvale, California, February 1969 .

III-6 N. H. Abramson: The Dynamic Behavior of Liquids in Moving Containers. NASA SP-106. Southwest Research Institute, San Antonio, Texas, 1966.

III-7 D. W. Moore: "The Rise of a Gas Bubble in a Viscous liquid." Joumal of Fluid Mechanics, Vo1 2, No. 2, July 1959.

III-8 H. D. Mendelson: "The Prediction of Bubble Terminal Velocity from Wave Theory." AIChE Joumal, Vo1 13, March 1967.

III-9 S. G. Berenyi and K. L. Abdalla: Vapor Ingestion Phenomenon in Hemispherically Bottomed Tonks in Normal Gravity and in Weightlessness. NASA TN D-5704. NASA Lewis Research Center, Cleveland, Ohio, April 1970.

III-10 G. D. Bizzell, et al.: Low Gravity Draining from Hemispherically Bottomed Cylindrical Tanks. NASA CR-72718. Lockheed Missiles and Space Co., Sunnyvale, California, June 1970.

III-11 J. R. Tegart: Computer Modeling of Liquid Motion in Spacecraft. R-70-48661-004, Martin Marietta Corporation, Denver, Colorado, December 1970 .

III-12 J. R. Tegart and R. E. Hise: Correlation of Drop Tower Tests with MAC Model. R-71-48631-001, Martin Marietta Corporation, Denver, Colorado, May 1971.

III-13 J. D. Montgomery, et al.: Pressurization System for Use in the Apollo Service Propulsion System--Computer Program Utilization Manual. CR-66-39. Martin Marietta Corporation, Denver, Colorado, July 1966. 
III-14 H. L. Paynter, et al. : Investigation of Space Storable Propellant Acquisition Devices, Final Report, Volume I. MCR-70-171 (Vol I), Martin Marietta Corporation, Denver, Colorado, October 1970.

III-15 R. E. DeLand, et al.: Space Storable Propelzant Module Thermal Control Technology Summary Report, Volume II, $\mathrm{F}_{2} / \mathrm{N}_{2} \mathrm{H}_{4}$ Propulsion Module. Report No. 14051-6009-R0-00. TRW Systems Group, Redondo Beach, California, 15 March 1971.

III-16 H. S. Mickley, et al.: Applied Mathematics In Chemical Engineering. McGraw-Hill Book Company, Inc., New York, New York, 1957.

III-17 R. J. Roark: Formulas for Stress and Strain, Third Edition. McGraw-Hill, New York, New York, 1954.

III-18 P. E. Uney and D. A. Fester: Material Compatibility with Space Storable Propelzonts, Design Handbook. MCR-72-26. Martin Marietta Corporation, Denver, Colorado, March 1972.

III-19 Personal Communication between P. E. Uney and E. Cutrell, Martin Marietta Corporation, Denver, Colorado, May 1972.

III-20 Fluorine Systems Handbook. NASA CR-72064. Douglas Aircraft, Santa Monica, California, July 1967.

III-21 Metals Handbook - Vol 6, Welding and Brazing. American Society for Metals, Metals Park, Ohio, 1971.

III-22 S. M. Toy, et al.: "Studies of Galvanic Corrosion Couples in Liquid Fluorine." Corrosion, Vol 24, No. 12, pp. 418-421, December 1968 .

III-23 P. E. Bingham, et al.: Launch Operations with Upper Stages Containing Eluorine. MCR-72-83. Martin Marietta Corporation, Denver, Colorado, April 1972 .

Chapter V

V-1 H. L. Paynter, et al.: Investigation of Space Storable Propelzant Acquisition Devices, Final Report, Volume I. MCR-70-171 (Vol I), Martin Marietta Corporation, Denver, Colorado, October 1970.

V-2 R. Prydy, and G. C. Straty: "The Thermodynamic Properties of Compressed Gaseous and Liquid Fluorine." National Bureau of Standards Technical Note 392 , U. S. Government Printing office, Washington, D.C., October 1970. 
V-3 V. J. Johnson, General Editor: "A Compendium of the Properties of Materials at Low Temperature (Phase I), Part I, Properties of Fluids." WDD Technical Report 60-56, Part I, Wright Air Development Division, Air Research and Development Command, United States Air Force, Wright-Patterson Air Force Base, Ohio, October 1960.

V-4 R. D. McCarty and L. A. Weber: "Thermophysical Properties of Oxygen from the Freezing Liquid Line to $600 \mathrm{R}$ for Pressures to 5000 psia." National Burecu of Stondards Technical Note 384, U. S. Government Printing Office, Washington, D.C., July 1971.

V-5 J. S. Whittick, et al.: Physical Properties of Liquid Oxygen Difluoride and Liquid Diborone: A Critical Review Updated. Stanford Research Institute Report 951581-4A, Stanford Research Institute, Men 1o Park, California, September 30, 1969.

V-6 H. L. Paynter, et al.: Investigation of Space Storable Propellant Acquisition Devices, Final Report, Volume II. MCR-70-171 (Vol II). Martin Marietta Corporation, Denver, Colorado, December 1970 .

V-7 Engineering Property Data on Rocket PropelZants (U). AFRPL-TR68-100. Rocketdyne Division, North American Rockwell Corporation, Canoga Park, California, May 1968 (Confidential).

V-8 B. Kit and D. S. Evered: Rocket PropelZant Handbook. The Macmillan Company, New York, New York, 1960.

V-9 Liquid Propelzant Manual (U). Chemical Propulsion Information Agency, Silver Spring, Maryland, December 1966.

V-10 M. T. Constatine, et al.: Diborane Handbook. R-8248. Rocketdyne Division, North American Rockwell Corporation, Canoga Park, California, 1970 .

V-11 R. Byron Bird, et aZ.: Transport Phenomena. John Wiley and Sons, Incorporated, New York, New York, 1960.

Chapter VI

VI-1 Personal Communication between P. E. Uney and E. Cutre11, Martin Marietta Corporation, Denver, Colorado, May 1972.

VI-2 H. I. Paynter, et al.: Investigation of Space Storable Propellant Acquisition Devices, Fingal Report, Volume I. MCR-70-171 (Vo1 I), Martin Marietta Corporation, Denver, Colorado, October 1970.

VIII-4 
VI-3 Personal Communication between P. E. Uney and R. G. Peterson; Martin Marietta Corporation, Denver, Colorado, May 1972.

VI-4 H. L. Paynter, et al.: Investigation of Space Storable Propellant Acquisition Devices, Final Report, Volume II. MCR-70-171 (Vol II), Martin Marietta Corporation, Denver, Colorado, December 1970 .

VI-5 0. L. Scott: Heat Pipe Development Progrom. Summary Report TCM70-016. Martin Marietta Corporation, Denver, Colorado, September 1970 .

VI-6 P. E. Uney and D. A. Fester: Material Compatibility with Space Storable PropelZants, Design Handbook. MCR-72-26. Martin Marietta Corporation, Denver, Colorado, March 1972.

VI-7 Personal Communication between P. E. Uney and M. W. Meyer, Martin Marietta Corporation, Denver, Colorado, May 1972.

VI-8 C. D. Brown: Viking '75 Orbiter Propulsion Subsystem PropelZant Management Device Cleaning Study. Interim Report No. 1, Martin Marietta Corporation, Denver, Colorado, May 1972.

VI-9 L. J. Rose, et al.: Cryogenic Propulsion Ground Hold System (U). AFRPL-TR-71-53. Martin Marietta Corporation, Denver, Colorado, June 1971. (Contract F04611-70-C-0051) (Confidential).

VI-10 D. W. Murphy, et al.: Vent-Free Fluorine Feed System (U). AFRPLTR-67-323 (Vo1 I). Martin Marietta Corporation, Denver, Colorado, March 1968. (Contract F04611-67-C-0044) (Confidential).

VI-11 Advanced Moneuvering Propulsion, (Lockheed Propelzant Feed System Design), Volume I - Sumnary (U). AFRFL-TR-70-104. Rocketdyne, Canoga Park, California, September 1970 (Confidential).

VI-12 D. A. Fester, et a..: "Liquid Fluorine No-Vent Loading Studies." $J$ of Spacecraft and Rockets. Vol 7, No. 2, February 1970.

VI-13 J. B. Keough and A. H. Oldland: Investigation of Cryogenic Rupture Disc Design. MCR-72-146. Martin Marietta Corporation, Denver, Colorado, May 26, 1972 (Contract NAS3-14345). 
DISTRIBUTION LIST FOR FINAL REPORT

CONTRACT NAS2-6548

COPIES

RECIPIENT

DESIGNEE

)

NASA LEWIS RESEARCH CENTER

21000 BROOKPARK RD.

CLEVELAND, OHIO 44135

1

OFFICE OF TECHNICAL INFORIMATION

$(x)$

NASA MANNED SPACECRAFT CENTER

HOUSTON, TEXAS 77058

OFFICE OF TECHNICAL INFORMATICN

$(x)$

NASA MARSHALL SPACE FLIGHT CENTER

HUNTSVILLE, ALABAMA 35812

OFFICE OF TECHNICAL INFORMATION, MS-IP $(x)$

TECHNICAL LIERARY

DALE BURROWS S+E-ASTN-PJ $(X)$

TECHNOLOGY UTILIZATION OFFICE, MS-T (X)

NASA AMES RESEARCH CENTER

MOFFET FIELD, CALIF. 94035

PATENTS AND CONTRACTS MANAGEMENT $(X)$

1

A. S. HERTZOG, DIRECTOR OF PROCUREMENT

JET PROPULSION LABORATORY

4800 OAK GROVE DR.

PASADENA , CALIF. 91103

2

G. A. YANKURA

$(x)$

NASA HEADQUARTERS

WASHINGTON, D.C., 20546

3 MANAGER, LIQUID ROCKET PROPULSION TECH., CODE RPT $(x)$

3 MANAGER, SPACE STORABLE PROPULSION TECHNOLCGY, (X) CODE RPI

OFFICE OF ADVANCED RESEARCH AND TECHNOLOGY

NASA HEADQUARTERS

WASHINGTON, D.C. 20546

1 DIRECTOR, TECHNOLOGY UTILIZATION DIVISION

$(x)$

OFFICE OF TECHNOLOGY UTILIZATION 
25 NASA SCIENTIFIC AND TECHNICAL INFORMATION FACILITY (X)

P.O. BOX 33

COLLEGE PARK, MARYLAND 20740

1 DIRECTOR, LAUNCH VEHICLES AND PROPULSION, SV OFFICE OF SPACE SCIENCE AND APPLICATIONS

$(x)$

NASA HEAOQUARTERS

WASHINGTON, D. C. 20546

1 DIRECTOR, ADVANCED MANNED MISSIONS, MT

OFFICE OF MANNED SPACE FLIGHT

$(x)$

NASA HEADQUARTERS

WASHINGTON, D. C. 20546

1 MISSION ANALYSIS DIVISION

NASA AMES RESEARCH CENTER

MOFFETT FIELD, CALIFORNIA 24035

NASA FIELD CENTERS

2 AMES RESEARCH CENTER

MOFFETT FIELD, CALIFORNIA 94035

1 GODDARD SPACE FLIGHT CENTER

GREENBELT, MARYLAND 20771

2 JET PROPULSION LABORATORY

CALIFORNIA INSTITUTE OF TECHNOLOGY

4800 OAK GROVE DRIVE

PASADENA, CALIFORNIA 91103

2 JOHN F. KENNEDY SPACE CENTER, NASA

COCOA BEACH, FLORIDA 32931

2 LANGLEY RESEARCH CENTER

LANGLEY STATION

HAMPTON, VIRGINIA 23365

2 LEWIS RESEARCH CENTER

21000 BROOKPARK ROAD

CLEVELAND, OHIO 44135

2 MARSHALL SPACE FLIGHT CENTER

HUNTSVILLE, ALABAMA 35812

2 MANNED SPACECRAFT CENTER

HOUSTON, TEXAS 77058
HANS M. MARK

MERLAND L. MOSESON
CODE 620

HENRY OURLAGE, JR

PROPULSION DIV. 38

DR. KURT H. DEBUS

ED CORTWR IGHT

DIRECTOR

DIRECTOR

HANS G. PAUL

CODE R-P+VED

J.G. THIBODAUX, JR.

CHIEF, PROP. + POWER DIV. H. POHL

GOVERNMENT INSTALLATIONS

1 HEADQUARTERS, U.S. AIR FORCE

WASHINGTON 25, D.C. 20546

COL.C.K. STAMBAUGH

AFRST

A-2 
1 ARNOLD ENGINEERING DEVELOPMENT CENTER DR. H•K. DOETSCH ARNOLD AIR FORCE STATION TULLAHOMA, TENNESSÉE 37388

2 AIR FORCE ROCKET PROPULSION LAGORATORY RPRPD/MR. H. MAIN RESEARCH AND TECHNOLOGY DIVISION

AIR FORCE SYSTEMS COMMAND EDWARDS, CALIFORNIA 93523

1 AIR FORCE MISSILE TEST CENTER HOL.LOMAN AIR FORCE BASE NEW MEXICO 45433

LIBRARY

1 AIR FORCE MISSILE TEST CENTER PATRICK AIR FORCE. BASE, FLORIDA

L.J. ULLIAN

1 AERONAUTICAL SYSTEMS DIVISION AIR FORCE SYSTEMS COMMAND WRIGHT-PATTERSON AIR FORCE BASE DAYTON, OHIO 45433

1 SPACE AND MISSILE SYSTEMS ORGANIZATION AIR FORCE UNIT POST OFFICE LOS ANGELES 45, CALIFORNIA 90045

COL. CLARK TECHNICAL DATA CENTER

1 DEFENSE DOCUMENTATION CENTER HEADQUARTERS CAMERON STATION, BUILDING 5 5010 DUKE STREET

ALEXANDRIA, VIRGINIA 22314

ATTN- TISIA

1 BUREAU OF NAVAL WEAPONS

DEPARTMENT OF THE NAVY

WASHINGTON D. C. 20546

D.L. SCHMIDT

CODE ASRCNC-2

\section{WASHINGTON D. C. 20546}

1 U.S. NAVAL ORDNANCE TEST STATION CHINA LAKE

CAL IFORNIA $\$ 3557$

J. KAY

RTMS-41

1 PICATINNY ARSENAL

DOVER, NEW JERSEY 07801

1 U.S. ARMY MISSILE COMMAND REOSTONE ARSENAL

ALABAMA 35809

CODE 4562

CHIEF, MISSILE PROPULSION DIV.

I. FORSTEN, CHIEF LIQUID PROPULSION LABORATORY, MR. WALTER WHARTON

\section{CPIA}

1 CHEMICAL PROPULSION INFORMATION AGENCY APPLIED PHYSICS LABORATORY

8621 GEORGIA AVENUE

SILVER SPRING, MARYLAND 20910

TOM REEDY 
1 AEROJET-GENERAL CORP. 601 PLACENTIA AVE.

FULLERTON, CA 92631

1 AEROJET-GENERAL CORPORATION

P. O. BOX 296

AZUSA, CALIFORNIA 91703

1 AEROJET-GENERAL CORPORATION

P. O. BOX 1947

TECHNICAL LIBRARY, BLDG 2015, DEPT • 2410

SACRAMENTO, CALIFORNIA 95809

AEROJET-GENERAL CORPORATION

1 SPACE DIVISION

9200 EAST FLAIR DR

EL MONTE, CALIFORNIA 91734

1 AEROSPACE CORPORATION

2400 EAST EL SEGUNDO BOULEVARD

P. O. BOX 95085

LOS ANGELES, CALIFORNIA 90045

1 AVCO SYSTEMS DIVISION

WILMINGTON, MASSACHUSETTS

1 BEECH AIRCRAFT CORPORATION

BOULOER DIVISION

BOX 631

BOULDER, COLORADO

1 BELL AEROSYSTEMS COMPANY

P.O. BOX 1

BUFFALO, NEW YORK 14240

1 BELLCOMM

955 L-ENFANT PLAZA, S. W.

WASHINGTON, D. C.

1 BENDIX SYSTEMS DIVISION

BENDIX CORPORATION

3300 PLYMOUTH ROAD

ANN ARBOR, MICHIGAN 48105

1 BOEING COMPANY

P. O. BOX 3999

SEATTLE, WASHINGTON 98124

1 BOEING COMPANY

P. O. BOX 1680

HUNTSVILLE, ALABAMA 35801

1 COMSAT

1835 *K* ST. N.W.

WASHINGTON, D.C. 20036
W. D. PETERS

W. L. ROGERS

R. STIFF

S. MACHLAWSKI

JOHN G. WILDER

MS -2293

HOWARD B. WINKLER

J. H. RODGERS

W. M. SMITH

H. S. LONDON

JOHN M. BRUEGER

LIBRARY

TED SNOW

G. HUSON

A-4 
1 MISSILE DIVISION

CHRYSLER CORPORATION

P. O. BOX 2628

DETROIT, MICHIGAN 48231

I WRIGHT AERONAUTICAL DIVISION

CURTISS-WRIGHT CORPORAT ION

WOOD-RIDGE, NEW JERSEY 07075

1 DYNATECH CORPORATION

17 TUDOR ST.

CAMER IDGE, MASS. 02139

1 RESEARCH CENTER

FAIRCHILD HILLER CORPORATION

GERMANTOWN, MARYLAND

1 REPUBLIC AVIATION CORPORATION

FAIRCHILD HILLER CORPORATION

FARNINGOALE, LONG ISLAND, NEW YORK

1 GENERAL DYNAMICS, CONVAIR OIVISION

P. O. BOX 1128

SAN DIEGO, CALIFORNIA

1 MISSILE AND SPACE SYSTEMS CENTER

GENERAL ELECTRIC COMPANY

VALLEY FORGE SPACE TECHNOLOGY CENTER

P.O. BOX 8555

PHILADELPHIA, PA.

1 GRUMMAN AIRCRAFT ENGINEERING CORP.

BETHPAGE, LONG ISLAND

NEW YORK 11714

1 HELLER ASSOCIATES

418 S. WESTGATE

LOS A.NGELES, CA 90049

I HONEYWELL, INC.

AEROSPACE DIV.

2600 RIDGWAY RD

MINNEAPOLIS, MINN.

1 HUGHES AIRCRAFT CO.

AEROSPACE GROUP

CENTINELA AND TEALE STREETS

CULVER CITY, CALIF. 90230

I WALTER KIDDE AND COMPANY, INC.

AEROSPACE OPERATIDNS

567 MAIN STREET

BELLEVILLE, NEW JERSEY

1 LING-TEMCO-VOUGHT CORPORATION

P. O. BOX 5907

DALLAS, TEXAS, 75222
MR. JOHN GATES

G. KELLEY

$R \cdot F \cdot J O H N S O N$

RALPH HALL

LIBRARY

LIBRARY

F. MEZGER

F. E. SCHULTZ

JOSEPH GAVIN

WM. HELLER

MR. GCRDON HARMS

E. H. MEIER

V.P.AND DIV. MGR •,

RESEARCH + DEV. DIV.

R. J. HANVILLE

DIR. OF RESEARCH ENGR. 
1 ARTHUR D. LITTLE, INC.

20 ACORN PARK

LI BRARY

CAMBRIDGE, MASSACHUSETTS 02140

1 LOCKHEED MISSILES AND SPACE CO.

S. DE BROCK

ATTN-TECHNICAL INFORMATION CENTER

P.O. BOX 504

SUNNYVALE, CALIFORNIA 94088

1 LOCKHEED PROPULSION COMPANY

P. O. BOX 111

REDLANDS, CALIFORNIA: 92374

1 THE MARQUARDT CORPORATION

16555 SATICOY STREET

LIBRARY

VAN NUYS, CALIF. 91409

1 BALTIMORE DIVISION

MARTIN MARIETTA CORPORATION

BALTIMORE, MARYLAND 21203

1 DENVER DIVISION

MARTIN MARIETTA CORPORATION

P. O. BOX 179

DENVER, COLORADO 80201

1 ORLANDO DIVISION

MARTIN MARIETTA CORP.

BOX 5837

ORLANDO, FLORIDA

1 MISSILE AND SPACE SYSTEMS DIVISION MCOONNELL-DOUGLAS AIRCRAFT CO. 3000 OCEAN PARK BLVD.

SANTA MONICA, CA 90406

1 ASTROPOWER LABORATORY

MCDONNELL-DOUGLAS AIRCRAFT CO.

LIBRARY

MR. JOHN CALATHES (3214)

2121 PAULARINO

NEWPORT BEACH, CA 92663

1 MCDONNELL-DOUGLAS CORP.

P. O. BOX 516

MUNICIPAL AIRPORT

ST. LOUIS, MISSOURI 63166

1 SPACE + INFORMATION SYSTEMS DIVISION NORTH AMERICAN ROCKWELL

12214 LAKEWOOD BOULEVARD

DOWNEY, CALIFORNIA 90241

1 ROCKETDYNE (LIBRARY 586-306)

6633 CANOGA AVENUE

CANOGA PARK, CALIF. 91304

J. U. LaFRANCE

C. G. SKARTVEDT

J. FERM

R. W. HALLET

CHIEF ENGINEER

ADV. SPACE TECH.

DR. GEORGE MOC

DIRECTOR, RESEARCH

R. A. HERZMARK

1 NORTHROP SPACE LABORATORIES

3401 WEST BROANWAY

LIBRARY

HAWTHORNE, CALIFORNIA 90250

DR. R. J. THOMPSON

S. F. IACOBELLIS

DR. WILLIAM HOWARD

A-6 
PHILCO CORPORATION

FORD ROAD

NEWPORT BEACH, CALIFORNIA 92663

1 ASTRO-ELECTRONICS DIVISION

RADIO CORPORATION OF AMERICA

PRINCETON, NEW JERSEY 08540

1 ROCKET RESEARCH

YORK. CENTER

REDMOND, WASHINGTON 98052

1 STANFORD RESEARCH INSTITUTE

333 RAVENSWOOD AVENUE

$Y \cdot B R I L L$

D. BALZER

MENLO PARK, CALIFORNIA 94025

F. MCCULLOUGH, JR.

1 SUNSTRAND AVIATION

2421 1ITH STREET

ROCKFORD, ILLINOIS 61101

1 TRW SYSTEMS GROUP

TRW I NCORPORATED

ONE SPACE PARK

REDONDO BEACH, CALIF. 90278

DR. GERALD MARKSMAN

1 TAPCO DIVISION

23555 EUCLID AVENUE

CLEVELAND, OHIO 44117

1 THIOKOL CHEMICAL CORPORATION

HUNTSVILLE DIVISION

HUNTSVILLE, ALABAMA 35807

1 RESEARCH LABORATURIES

R. W. REYNOLDS

G.W. ELVERUM

UNITED AIRCRAFT CORP.

400 NAIN ST.

EAST HARTFORD, CONN. 06108

1 HAMILTON STANDARD DIVISON

UNITED AIRCRAFT CORP.

WINDSOR LOCKS, CONN. 06096

1 UNITED TECHNOLOGY CENTER

587 NIETHILOA AVENUE

P. O. B.OX 358

SUNNYVALE, CALIFORNIA 94088

1 FLORIDA RESEARCH AND DEVELOPMENT

PRATT AND WHITNEY AIRCRAFT

P. T. ANGELL

UNITED AIRCRAFT CORPORATION

P. O. BOX 2691

WEST PALM BEACH, FLORIDA 33402

1 VICKERS, INC.

BOX 302

TROY, MICHIGAN

ELKTON DIVISION

ERISTOL, PENNSYLVANIA

JOHN GOODLOE

ERLE MARTIN

$M R \cdot R \cdot H A T C H$

DR. DAVID ALTMAN

R.J. COAR

LI BRARY 